\title{
Lipid dependent interactions of biomimetic membrane models with the ENTH domain of epsin
}

\author{
Dissertation \\ for the award of the degree \\ Doctor rerum naturalium \\ of the Georg-August-Universität Göttingen \\ within the doctoral program \\ Physics of Biological and Complex Systems \\ of the Georg-August University School of Science (GAUSS) \\ Submitted by \\ Nelli Teske \\ born in Karabulak
}

Göttingen 2019 


\section{Members of the thesis committee}

Prof. Dr. Claudia Steinem

Institute of Organic and Biomolecular Chemistry

Georg-August-Universität Göttingen

Prof. Dr. Thomas Burg

Biological Micro- and Nanotechnology

Max-Planck-Institute for Biophysical Chemistry

Prof. Dr. Michael Meinecke

Department of Cellular Biochemistry

University Medical Center Göttingen

\section{Members of the examination board}

Prof. Dr. Claudia Steinem

Institute of Organic and Biomolecular Chemistry

Georg-August-Universität Göttingen

Prof. Dr. Thomas Burg

Biological Micro- and Nanotechnology

Max-Planck-Institute for Biophysical Chemistry

Prof. Dr. Michael Meinecke

Department of Cellular Biochemistry

University Medical Center Göttingen

\section{Further members of the examination board}

Prof. Dr. Bert de Groot

Computational Biomolecular Dynamics Group

Max Planck Institute for Biophysical Chemistry

Prof. Dr. Burkhard Geil

Institute of Physical Chemistry

Georg-August-Universität Göttingen

Dr. Sebastian Kruss

Institute of Physical Chemistry

Georg-August Universität Göttingen

Date of oral examination: 09 $9^{\text {th }}$ December 2019 
Declaration

I, Nelli Teske, hereby certify that my doctoral thesis entitled „Lipid dependent interactions of biomimetic membrane models with the ENTH domain of epsin" has been written independently and with no other source and aids than quoted.

Göttingen, 2019

Nelli Teske 

Meiner Familie 



\section{Content}

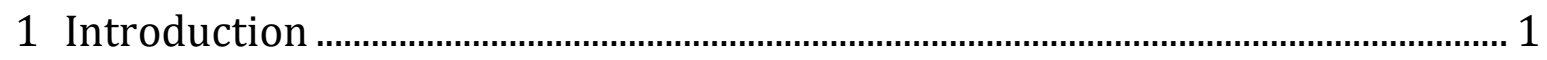

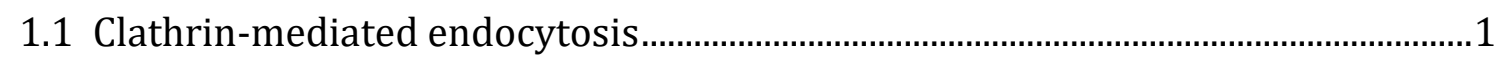

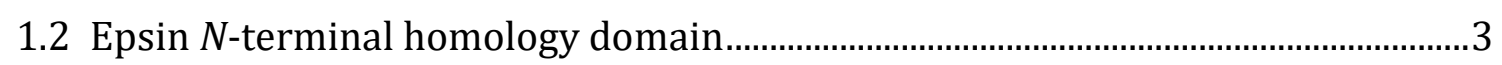

1.3 Interplay of the ENTH assembly and amphiphilic helix insertion ............................5

1.4 Lipid dependent membrane deformation .................................................................

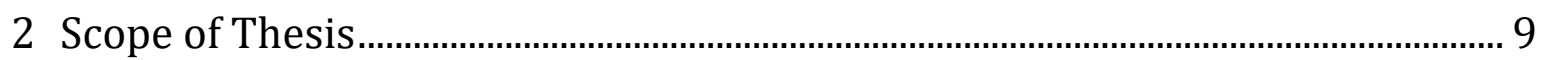

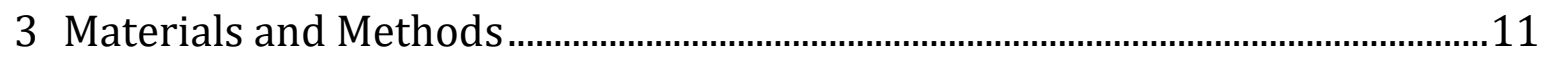

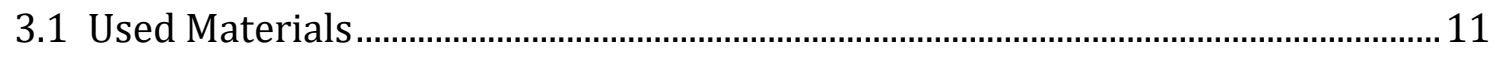

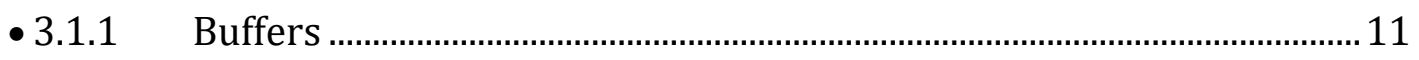

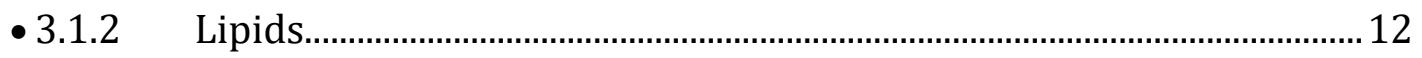

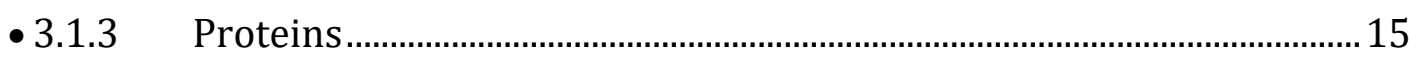

3.1.3.1 Epsin $N$-terminal homology domain................................................ 15

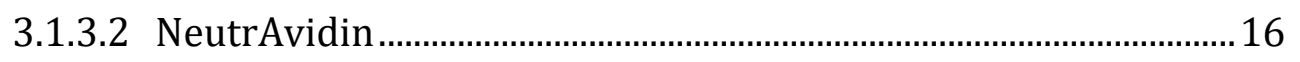

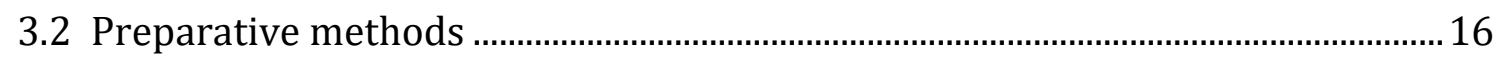

- 3.2.1 Preparation of unilamellar vesicles .......................................................... 16

• 3.2.2 Substrate surface preparation ................................................................. 18

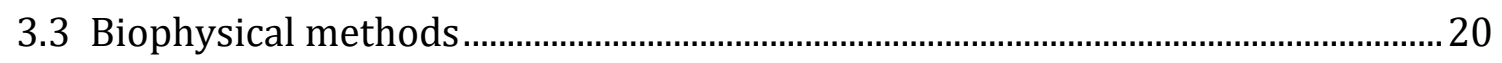

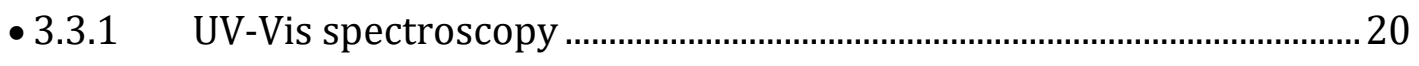

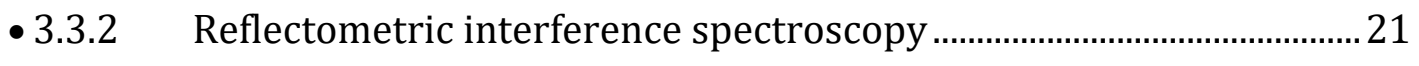

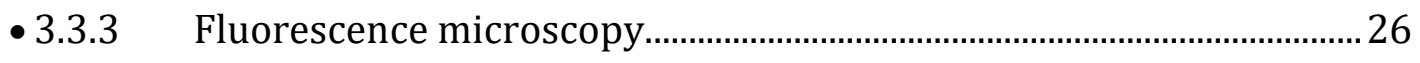

3.3.3.1 Confocal laser scanning microscope.............................................27

3.3.3.2 Spinning Disc ...................................................................................... 31

• 3.3.4 Atomic force microscopy

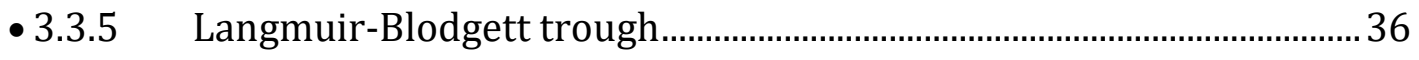

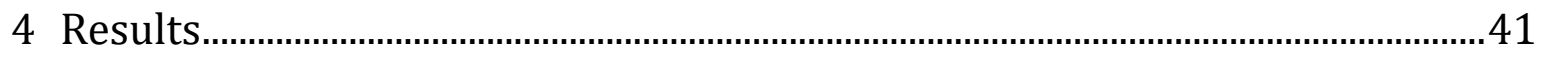

4.1 Influence of the PIP 2 concentration on the ENTH binding to solid supported lipid membranes. 
-4.1.1 Formation of solid-supported lipid bilayers on silicon dioxide......... 42

-4.1.2 Formation of solid-supported lipid monolayers on silicon dioxide.46

-4.1.3 Analysis of the ENTH binding on lipid mono- and bilayers. 50

4.2 The influence of PS on the ENTH binding to PIP2 containing solid supported lipid bilayers. 56

-4.2.1 Influence of PS on the bilayer formation .................................................. 56

-4.2.2 Influence of PS on the binding affinity of ENTH to PIP2 ....................... 58

-4.2.3 Topographical analysis of ENTH cluster formation ............................... 62

• 4.2.4 Investigation of the ENTH R114A mutant binding to PIP 2 .................. 67

4.3 Investigation of the ENTH-PIP2 interactions on lipid monolayers ......................... 72

• 4.3.1 $\mathrm{PIP}_{2}$ influence on the ENTH penetration ability .................................... 72

• 4.3.2 PS influence on the ENTH penetration ability ........................................ 79

4.4 Adhered GUVs as a membrane model to investigate protein-membrane interactions

-4.4.1 Theoretical model .84

- 4.4.2 Adhesion of biotinylated GUVs on PEGylated surfaces 88

-4.4.3 Effect of ENTH addition to adhered GUVs 94

5 Discussion 97

5.1 Characterization of the ENTH binding to $\mathrm{PIP}_{2}$ containing solid supported membranes.

-5.1.1 Asymmetric distribution of $\mathrm{PIP}_{2}$ in solid supported lipid bilayers and the influence on ENTH binding. 98

- 5.1.2 PS dependent binding affinity of ENTH to lipid bilayers 104

5.1.2.1 The ENTH R114A mutant 113

5.2 Lipid dependent penetration ability of ENTH to lipid monolayers 116

-5.2.1 Receptor lipid dependent penetration ability of ENTH 117

- 5.2.2 PS dependent penetration ability of ENTH

5.3 Adhered GUV as a membrane model to investigate protein-membrane interactions 
-5.3.1 Adhesion of biotinylated GUVs on PEG-functionalized surfaces .... 125

- 5.3.2 Effect of the ENTH binding to PS-containing adhered GUVs............ 129

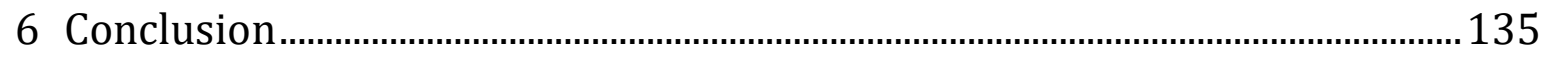

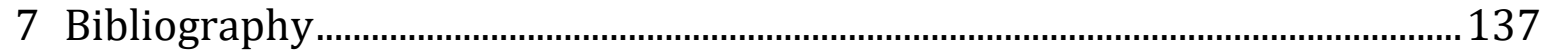

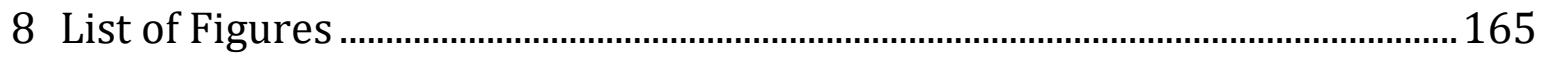

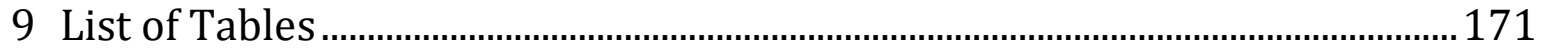

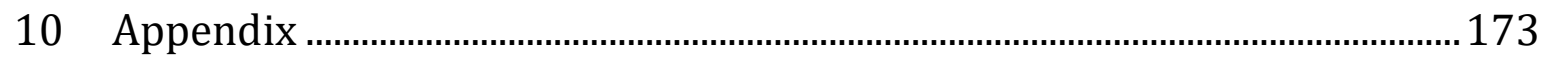


ABSTRACT. Membrane remodeling processes during endocytosis are highly regulated by the protein-protein and protein-membrane interactions. In clathrin-mediated endocytosis (CME) binding of the protein epsin to its receptor lipid phosphatidylinositol$(4,5)$-bisphosphate $\left(\mathrm{PIP}_{2}\right)$ induces the deformation of the membrane. Upon binding of the epsin $N$-terminal homology domain (ENTH) to PIP2, conformational changes in the protein lead to a newly formed helix, which inserts into the cytosolic leaflet.

By using artificial membrane models the binding affinity and the surface topology upon ENTH binding as a function of the lipid composition were analyzed. With increasing the $\mathrm{PIP}_{2}$ concentration the experiments on solid supported lipid bi- and monolayers proved a direct relation between the protein occupancy and the lipid content. Subsequently, this indicates that an accumulation of $\mathrm{PIP}_{2}$ on the cytosolic leaflet can facilitate the endocytosis rate.

Besides $\mathrm{PIP}_{2}$ also negatively charged lipids with the head group phosphatidylserine (PS) can affect protein binding in endocytosis. In presence of PS, higher binding affinities and protein occupancies of ENTH to PIP2 doped membranes were observed. Although ENTH is known to act as a monomeric protein, atomic force microscopy (AFM) measurements revealed the appearance of protein clusters induced by PS. Thus, also membrane crowding seems to have an impact on the curvature inducing step in CME. Mutation of the amino acid R114 showed its relevance in ENTH cluster formation as no oligomers were observed with the mutant R114A.

Moreover, monolayer penetration experiments were performed to analyze the surface activity of ENTH dependent on the lipid composition. Increasing the $\mathrm{PIP}_{2}$ content increased the critical surface pressure. Addition of PS did not significantly increase the penetration of ENTH into monolayers in a $\mathrm{PIP}_{2}$ dependent manner, although a higher protein occupancy on supported lipid bilayers was observed.

To investigate whether ENTH has an influence on mechanical properties of membranes in the presence of PS, giant unilamellar vesicles (GUVs) were adhered to NeutrAvidin coated surfaces. Incubation of these GUVs with ENTH resulted in the rupturing of those due to lipid packing defects in the membrane by helix insertion and PS induced clusters. These results allow to understand how PS alters the binding structure of ENTH to $\mathrm{PIP}_{2}$ doped membranes. This in turn also show that the lipid composition contributes to the regulation of protein-dependent membrane deformation during CME. 
ZUSAMMENFASSUNG. Während der Endozytose wird die Membran durch verschiedene Protein-Protein und Protein-Lipid Interaktionen reguliert. In der Clathrin-vermittelten Endozytose (CME) bewirkt die Bindung des Proteins Epsin an sein Rezeptorlipid Phosphatidyl-(4,5)-bisphosphat ( $\left.\mathrm{PIP}_{2}\right)$ eine Membrankrümmung. Durch diese Bindung wird in der Epsin $N$-terminalen Homologie Domäne (ENTH) eine neue Helix $\left(\alpha_{0}\right)$ ausgebildet, die die cytosolische Lipidmonoschicht der Plasmamembran penetriert. Mit artifiziellen Membransystemen konnten die Bindungsaffinitäten und die Oberflächentopologien bei ENTH-Anbindung in Abhängigkeit der Lipidzusammensetzung analysiert werden. Mit zunehmender PIP2-Konzentration zeigten die Experimente an fest unterstützten Lipiddoppelschichten und -monoschichten einen direkten Zusammenhang zwischen der Proteinbelegung und der Rezeptorlipidkonzentration. Dies wiederum deutet darauf hin, dass eine Anhäufung von $\mathrm{PIP}_{2}$ in der Plasmamembran die Endozytose-Rate steigern kann.

Nicht nur bei der Erhöhung der PIP2-Konzentration wurde eine höhere Proteinoberflächenbelegungen auf Membranen beobachtet, sondern auch in Gegenwart der Lipidkopfgruppe Phosphatidylserin (PS), welche die Bildung von ENTH Cluster induziert. Somit scheint neben der Helixinsertion auch das Zusammenlagern mehrerer ENTHMoleküle (Crowding) einen Einfluss auf den krümmungsinduzierenden Schritt in der CME zu haben. Mit der Mutante ENTH R114A konnte zudem gezeigt werden, dass die Aminosäure R114 essentiell für die Clusterbildung ist.

Penetrationsexperimente an Lipidmonoschichten wurden durchgeführt, um die Oberflächenaktivität von ENTH in Abhängigkeit von der Lipidzusammensetzung zu analysieren. Dabei zeigte die PIP 2 Konzentration einen größeren Einfluss auf den kritischen Oberflächendruck als die Zugabe von PS.

Adhärierte Riesenvesikel (GUVs) ermöglichten zudem die mechanischen Eigenschaften von Membranen in Abhängigkeit von PS zu untersuchen. Inkubation dieser GUVs mit ENTH führte zum Rupturieren der Vesikel durch die Entstehung von Defekten während der Helixinsertion und der PS-induzierten Clusterbildung. Dies zeigt, dass PS einen deutlichen Einfluss auf die Bindungsstruktur von ENTH an PIP2 Membranen hat. Dadurch konnte auch gezeigt werden, dass die Lipidzusammensetzung zur Regulation der proteinabhängigen Membrankrümmung während der CME beiträgt. 



\section{INTRODUCTION}

Cells are complex systems, consisting of different cell organelles with various functions. The cell membrane surrounds the cytoplasm of the cell and serves as protection from the environment. This barrier function enables the selective control of cell communication and molecule exchange. Both processes rely on substance transport, which can either be passive by simple diffusion or by carrier and channel proteins. Contrary to that, active transport requires energy to regulate the uptake of particles. However, large molecules are not able to pass the membrane even though the cell supplies the energy. To overcome this barrier, the transport of large molecules is driven by exo- and endocytosis.

\subsection{Clathrin-mediated endocytosis}

One important and well known endocytotic pathway is the clathrin-mediated endocytosis (CME). Here macromolecules are internalized into vesicles derived from the plasma membrane. The receptor regulated process enables the controlled entry into cells, which is important for immune response or intercellular communication. ${ }^{1,2}$ The process is connected to highly coordinated and complex interactions, preventing the entry of unwanted viruses or toxins. Although under certain conditions, like dysfunctions, they can gain entry and cause serious diseases. ${ }^{3,4}$

Since clathrin does not have a binding motif for the membrane, adaptor proteins connect clathrin with membrane lipids and other proteins. ${ }^{5}$ The uptake of cargo-molecules is driven by a complex system of proteins, proceeded in five steps.

By membrane binding of accessory proteins first a curvature of the bilayer occurs, followed by the cargo selection via receptor molecules. Then the recruitment of the clathrin triskelia molecules induces the polymerization of clathrin, forming a stable cage around the invagination (clathrin-coated pit). ${ }^{6-8}$ Scission trough proteins, like dynamin, ${ }^{9,10}$ leads to the formation of the clathrin-coated vesicle (CCV). In the last 
step, the clathrin triskelia cage decays and consequently the cargo molecules are released. This five step mechanism of the clathrin-mediated endocytosis is illustrated in Figure 1.1.

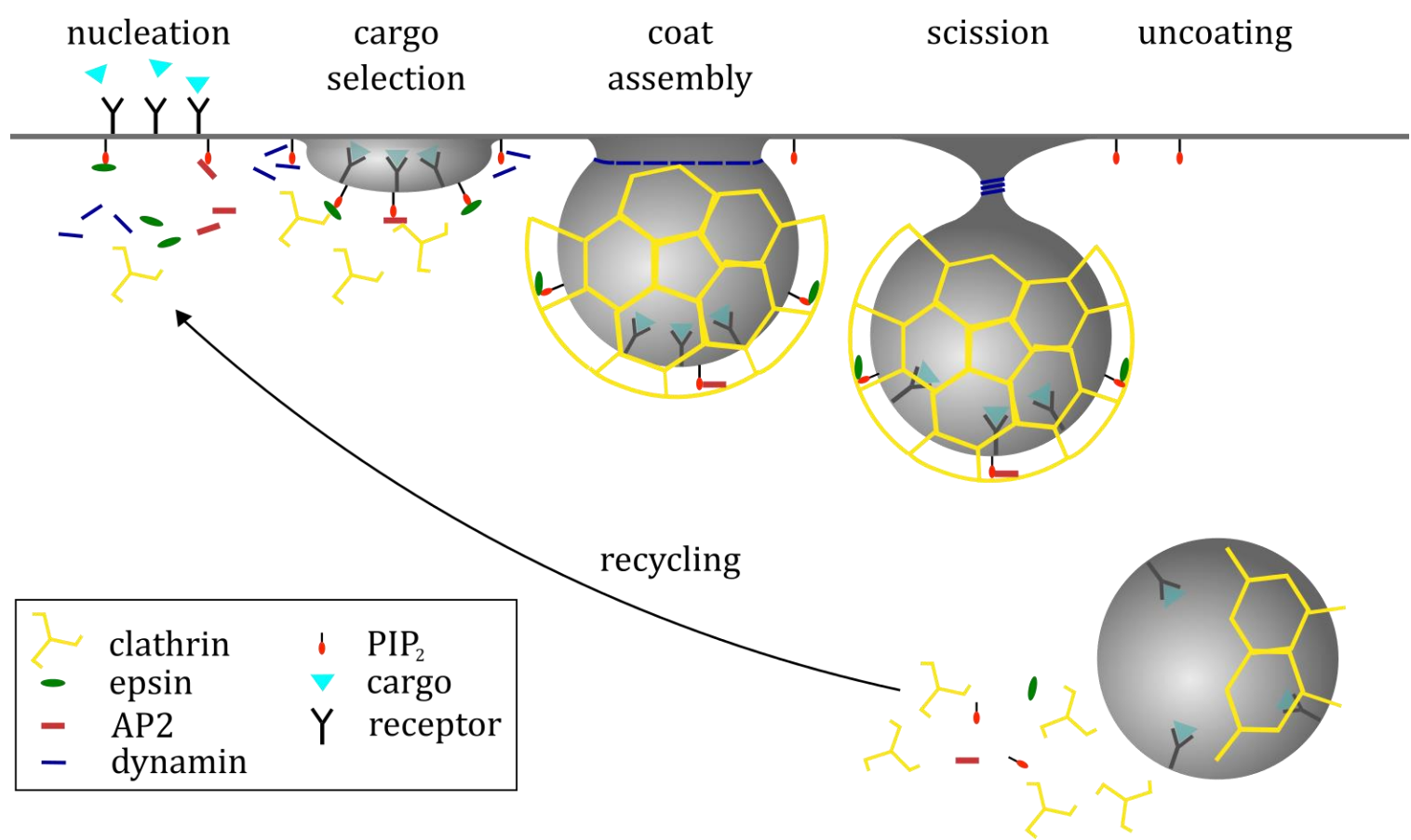

Figure 1.1: Schematic illustration of the clathrin-mediated endocytosis. Binding of proteins like epsin and AP2 to the receptor lipid $\mathrm{PIP}_{2}$ induces an invagination, during which cargo molecules are recruited by receptor proteins. Afterwards polymerization of clathrin leads to a curvature stabilizing cage. Due to further curvature and coat assembly a vesicle is formed. Scission and uncoating result in a free vesicle, which is transported to the target cell compartment. Figure modified according to Messa et al. ${ }^{11}$

The cargo uptake is highly regulated by receptor proteins. Adaptors like AP2, AP180/CALM and epsin are noteworthy as they exhibit binding sites for cargo molecules and bind clathrin as well as the membrane. ${ }^{12-14}$ Dephosphorylation of phosphatidylinositol-(4,5)-bisphosphate ( $\left.\mathrm{PIP}_{2}\right)$ by synaptojanin induces a decreased binding to clathrin associated proteins, which destabilizes the clathrin coat and consequently results in uncoating. ${ }^{15}$ Uncoated vesicles typically are transported to early endosomes, where they fuse and release their cargo. ${ }^{16}$ Thereafter, the receptors are recycled and recruited to be available for the next CME circle. The role of various proteins in CME is still not completely understood. ${ }^{17}$ Nevertheless, all membrane-interacting adaptors in CME seem to have at least two functions. Cargo selecting receptors with 
the ability to generate membrane curvature ensure the vesicle formation independent from the cargo molecule. ${ }^{1}$ One molecule, which is crucial for membrane reorganization during CME is epsin.

\subsection{Epsin $N$-terminal homology domain}

The accessory protein epsin is among other clathrin-associated sorting proteins essential for the CME. It was first discovered in 1998 by Chen et al. as Eps15 interacting protein (epsin). ${ }^{18}$ Epsin 1 and the three isoforms are present in all vertebrates. ${ }^{7}$ Other epsin-like homologues were also found in lower species. ENTH contains several binding motifs, important for different functions. For example, it is essential in signaling activation of the epidermal growth factor receptor (EGFR), contributed in cancer diseases, or the epithelial sodium channel as it regulates electrolyte balances. The $C$-Terminus of epsin has a binding site for proteins like Eps15 and intersectin. ${ }^{18}$ Further motifs in the protein can interact with ubiquitin and AP2 (Figure 1.2). ${ }^{19,20}$

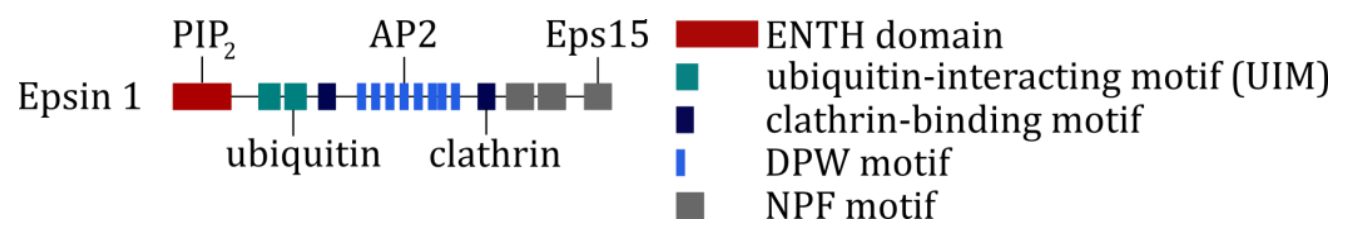

Figure 1.2: Schematic structure of epsin 1 with all important binding motifs, necessary for interactions with $\mathrm{PIP}_{2}$, ubiquitin, AP2, clathrin and Eps15. Derived from Horvath et al. ${ }^{7}$

In the CME epsin is recruited to the plasma membrane by the evolutionary well conserved epsin $N$-terminal homology (ENTH) domain. ${ }^{20}$ It consists of about 150 amino acids structured to a superhelix of seven $\alpha$-helices. At the plasma membrane the domain specifically recognizes and binds the receptor lipid $\mathrm{PIP}_{2}$, resulting in a conformational change in the tertiary structure of its ENTH domain. This however leads to the formation of a $\mathrm{PIP}_{2}$ binding pocket established by the amino acids R7, R8, K11, R25, R63, K69 and H73 of the ENTH domain, which accommodates the negative charge of the receptor lipid. ${ }^{8,21}$ An unstructured 14 amino acids large sequence of the domain becomes ordered, called the $\alpha_{0}$ helix. The newly formed helix inserts into the 
inner leaflet of the membrane, reduces the energy barrier of bending and consequently modifies membrane curvature. Ford et al. were able to co-crystallize the ENTH domain together with inositol-(1,4,5)-triphosphate (IP3), the head group of $\mathrm{PIP}_{2}$ (Figure 1.3) ${ }^{22}$, showing the structural change in the protein.

A

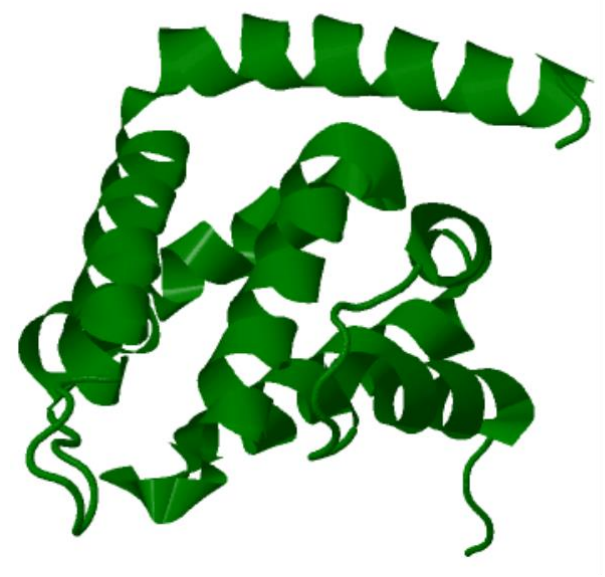

B

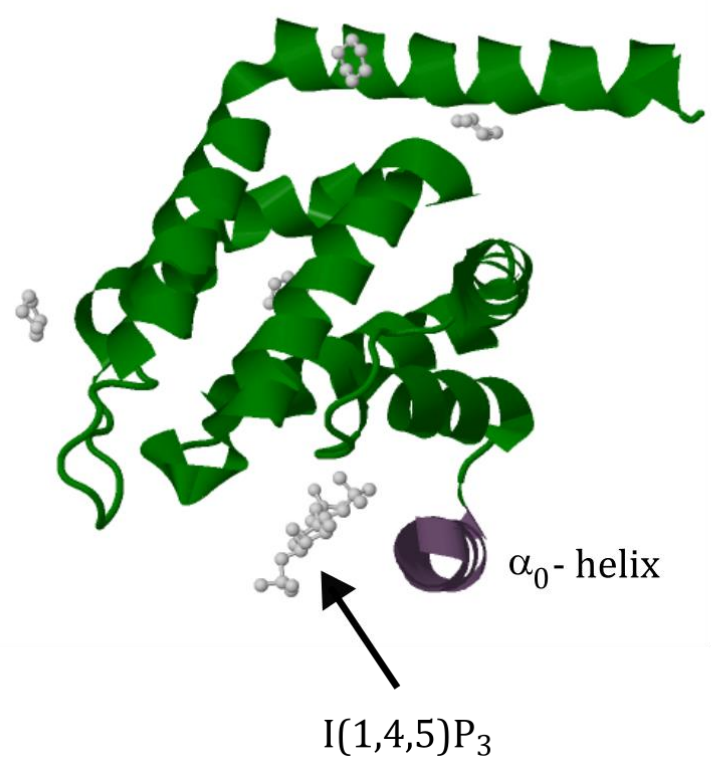

Figure 1.3: Crystal structures of unbound ENTH and the ENTH-IP 3 complex. Binding to $\mathrm{IP}_{3}$ leads to the formation of a new helix $\left(\alpha_{0}\right)$. In the bound state the R114 loop of ENTH is in close contact to the membrane. 22,23

Itoh et al. showed that the ENTH-PIP2 binding is specific as the affinity to other inositol species and phospholipids was drastically decreased or missing. ${ }^{24}$ Moreover the binding affinity can be influenced by the $\mathrm{pH}$ or the lipid composition of the membrane. 8,25,26 The ENTH domain furthermore can induce tubulation in PIP2-containing liposomes and also tubular structures in the plasma membrane of living cells. $21,27,28$ Besides the curvature inducing effect, epsin also binds to clathrin in the CME. ${ }^{29}$ It connects clathrin with the membrane and causes its polymerization, which stabilizes the invagination and the subsequent vesicle formation (cf. chapter 1.1).

The complete contribution and interaction of ENTH domain in the network of clathrin associated proteins is still discussed and thus the investigation is important. Some studies indicate that not only the insertion of the amphiphilic helix leads to a curvature, but also the assembly of the protein is contributing the membrane curvature process. ${ }^{30}$ 


\subsection{Interplay of the ENTH assembly and amphiphilic helix insertion}

Many biological processes depend on highly curved membrane structures, even though induction of curvature is associated with high energy requirements. To overcome this energy barrier an interplay between several proteins in collaboration with lipids is necessary. There are different ways to generate membrane deformation. The epsin ENTH domain is known to induce membrane asymmetry by the insertion of an amphiphilic helix. Ford et al. performed mutagenesis experiments, where they changed the hydrophobicity of the $\alpha_{0}$ helix on position L6. ${ }^{21}$ Decreased hydrophobicity resulted in lower binding affinity and decreased tubulation, showing the impact of the amphiphilic helix of ENTH.

Nevertheless, computational studies challenge the efficiency of helix insertion to induce membrane deformation. ${ }^{31,32}$ According to these studies, about $10-25 \%$ of the membrane surface have to be occupied by helices. Considering the footprint of ENTH, a physiological improbable coverage of almost $100 \%$ would be necessary. ${ }^{23} \mathrm{Am}$ phiphilic helix insertion induced deformation of membranes was also found for other proteins, but usually this effect was supported by the shape of the protein. An example is the $\mathrm{N}$-BAR domain of endophilin. ${ }^{33}$ The banana-shaped protein domain is enriched in synapses and stabilizes the helix induced membrane curvature by its curved shape. In contrast to that, ENTH does not show an inherent curvature itself. This leads to the question if an additional effect supports the helix insertion and promotes membrane curvature.

Stachowiak et al. assumed that crowding of ENTH drives membrane bending due to lateral pressure resulting from collision of two bound proteins. ${ }^{30}$ With increasing protein coverage the pressure increases until the energy barrier is reached to induce membrane deformation. They proved the relevance of the protein crowding mechanism with His-tagged ENTH lacking the $\alpha_{0}$ helix. Fluorescence microscopy experiments with giant unilamellar vesicles showed that high coverage with His-tagged ENTH also resulted in tubular structures, indicating the contribution of protein-protein interaction on the membrane deformation process. 
Contrary to that, Kozlov et al. exhibited with computational predictions the high relevance of the ENTH insertion mechanism. ${ }^{34}$ They calculated the membrane tube radius for different models, which predict that the crowding effect is clearly weaker than the insertion effect. Protein crowding as a non-specific interaction with the membrane would lead to bending on both sides of the membrane, thus counteract the effect on each side. Further studies investigated the protein crowding induced deformation, assuming that it is co-regulated by membrane tension and protein coverage. 35 Recent studies showed that binding of ENTH to PIP2-containing membranes resulted in the decrease of lateral membrane tension and consequently in a decreased bending modulus. ${ }^{36}$ Hence, an interplay of both, the protein crowding and the protein helix insertion of ENTH, seems likely to induce membrane curvature. In Figure 1.4 the predicted mechanism involved in ENTH induced membrane bending is illustrated.

A

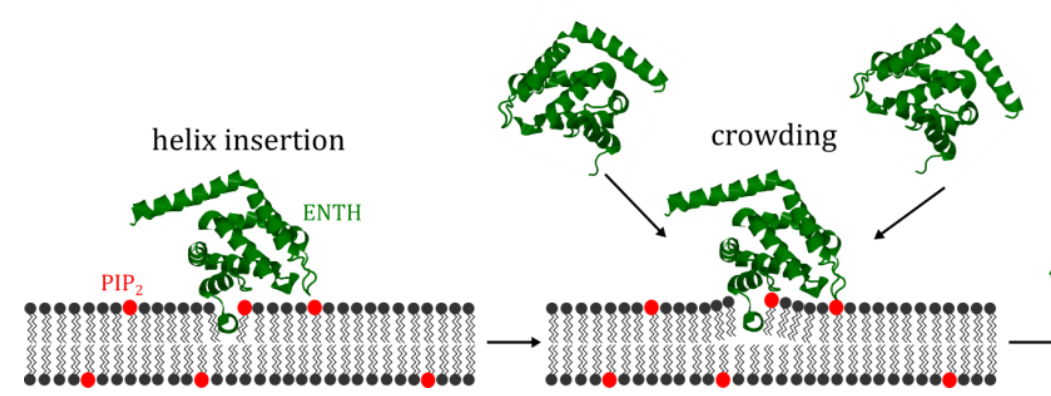

C

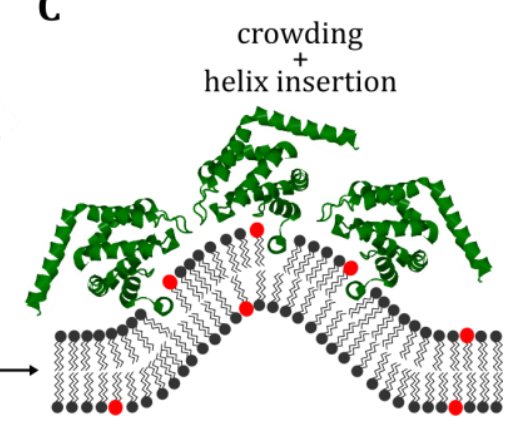

Figure 1.4: Schematic illustration of the predicted mechanism of ENTH induced membrane curvature. Recruitment of ENTH by PIP 2 results in the insertion of an amphipathic helix (A). The helix insertion of ENTH disturbs the structure of the lipid bilayer (B). Rearrangement leads to a deformation of the membrane. The assembly of several ENTH monomers facilitates further membrane curvature (C), which represents the first step in CME.

Besides the effect of proteins, also the lipid composition affects the membrane topology. Due to versatile lipid shapes, charges and intramolecular interactions, they can induce membrane asymmetries promoting membrane curvature. ${ }^{37}$ 


\subsection{Lipid dependent membrane deformation}

The plasma membrane consists of various lipids and proteins interacting with each other. Amphiphilic lipids have a hydrophilic head group and long hydrocarbon chain residues. On one hand the lipids in the membrane built the barrier, but additionally they also have other functions for living cells. They are necessary for the energy storage or as signal molecules. ${ }^{38,39}$ Furthermore, the lipid structure or geometry can influence the shape of the membrane due to different packing densities. ${ }^{40,41}$ If lipids have a large area ratio of head groups to acyl chains, they can create an invagination on the other side of the membrane. The lipids in the membrane are usually distributed asymmetrically, which arises from dynamic flip-flop and lateral diffusion processes. ${ }^{42,43}$ Among other effects, this asymmetry can lead to the deformation of the membrane. Certain proteins or molecules are localized in specific compartments of the cell. Asymmetric distribution or even crowding of the lipids in these regions would ensure the protein-lipid interactions, important for several processes. More often the relevance of lipids in membrane deformation is coupled to proteins, which induce this effect. One important lipid, which binds several proteins involved in membrane curvature is $\mathrm{PIP}_{2}$. In mammalian cells it is the most abundant phosphoinositide species as it incorporates $1 \%$ of all phospholipids, occurring in the plasma membrane. ${ }^{44}$ It is the source of the two second messengers diacylglycerol as well as inositol-1,4,5-triphosphate and also interacts with proteins important for the membrane's attachment to the cytoskeleton. Moreover, several studies revealed the essential role of $\mathrm{PIP}_{2}$ in exo- and endocytotic processes. ${ }^{45-48}$ For example, it is an important regulator of the BAR domain membrane deformation activity, required in endocytosis. In the CME it is also the major regulator for various clathrin associated proteins. Due to recruitment of AP2 and epsin, the membrane is bent until a vesicle is formed (cf. chapter 1.1). Also the uncoating of CCVs is regulated by $\mathrm{PIP}_{2}$, enabling the fusion of the vesicle with the membrane of the target cell compartment. ${ }^{15,49}$ Even though $\mathrm{PIP}_{2}$ is probably the most essential lipid involved in membrane deformation processes, also other lipids affect them. For example, phosphatidylserine (PS) lipids can influence these processes. They are highly enriched in the plasma membrane (12\%), ${ }^{37,50}$ especially in the cytosolic leaflet. The anionic lipid is involved in the coagulation cascade 
and also in the apoptosis process. ${ }^{51,52}$ Due to the negative charge it causes membrane deformation by the recruitment of proteins. In neuronal exocytosis $\mathrm{PIP}_{2}$ and PS bind synaptotagmin-1, which triggers fusion. ${ }^{53}$ In yeast, the absence of PS translocation leads to an insufficient membrane curvature as PS drives the assembly of involved proteins like clathrin. ${ }^{54}$ Furthermore, in CME it recruits proteins to the membrane and significantly affects the efficiency of this process. ${ }^{55}$

The exact influence of lipid compositions and their interplay with proteins in the CME is still poorly understood. To investigate such protein-membrane interactions often artificial membrane models were used to mimic biological membranes in a simplified way. This allows the analysis of the contribution of certain lipids in protein induced membrane deformation processes under controlled conditions. 


\section{SCOPE OF THESIS}

Endocytosis processes are regulated by interactions of several proteins and lipids. It enables the transport of important nutrients and signal molecules into cells. Thereby, the clathrin-mediated endocytosis (CME) represents the main pathway of endocytosis, thus the investigation is of great interest. During the CME adaptor proteins, like epsin, bind to the receptor lipid phosphatidylinositol-(4,5)-bisphosphate ( $\left.\mathrm{PIP}_{2}\right)$ and induce a membrane curvature. Upon specific binding of the epsin $N$-terminal homology (ENTH) domain to $\mathrm{PIP}_{2}$, a former unstructured region rearranges to a new ordered helix. Insertion of this helix induces asymmetry within the both bilayer leaflets and facilitates the membrane deformation. The influence of lipids in this mechanism is still not understood completely and will be characterized more precisely within the scope of the thesis.

Therefore, different methods will be used to analyze specific interactions of the ENTH with $\mathrm{PIP}_{2}$-doped artificial membranes as a function of the lipid composition. In particular the influence of the lipid receptor and the negatively charged lipid phosphatidylserine (PS) on binding affinities and the helix insertion of ENTH will be evaluated. Solid supported lipid membranes will be established to determine the surface occupancy and the dissociation coefficient of the ENTH domain. Thereby, the differences in the binding behavior to $\mathrm{PIP}_{2}$ containing bi- and monolayers will be analyzed as well as the influence of PS. To investigate if besides the helix insertion also a membrane crowding effect induces a membrane deformation, atomic force microscopy will give information about the surface topologies upon ENTH binding. Moreover, mutagenesis experiments moreover shall show the relevance of specific regions within the protein. Monolayer penetration experiments on a Langmuir-Blodgett trough will clarify how the lipid packing influences the helix insertion and consequently the membrane deformation. Finally, the impact of ENTH on mechanical properties of free-standing membranes in the presence of PS will be analyzed using adhered GUVs to understand the contribution of lipid compositions and membrane tension as regulators for membrane deformation processes. 



\section{Materials ANd Methods}

\subsection{Used Materials}

\subsubsection{Buffers}

For the experiments in this work different buffer systems were used. In Table 3.1 the compositions of these are listed. All buffers were diluted in ultrapure water, degased and filtered (pore size $\emptyset 0.2 \mathrm{~nm}$ ).

Table 3.1: Used buffers and their compositions.

\begin{tabular}{|c|c|}
\hline buffer & composition \\
\hline citrate buffer & $\begin{array}{l}\text { Na-citrate }(20 \mathrm{mM}) \\
\mathrm{KCl}(50 \mathrm{mM}) \\
\text { EDTA }(0.1 \mathrm{mM}) \\
\mathrm{NaN}_{3}(0.1 \mathrm{mM})\end{array}$ \\
\hline phosphate buffered saline (PBS), pH 7.4 & $\begin{array}{l}\mathrm{KCl}(0.3 \mathrm{mM}) \\
\mathrm{NaCl}(140 \mathrm{mM}) \\
\mathrm{KH}_{2} \mathrm{PO}_{4}(0.1 \mathrm{mM}) \\
\mathrm{Na}_{2} \mathrm{HPO}_{4}(10 \mathrm{mM})\end{array}$ \\
\hline protein buffer, $\mathrm{pH} 7.4$ & $\begin{array}{l}\mathrm{NaCl}(200 \mathrm{mM}) \\
\text { HEPES }(10 \mathrm{mM})\end{array}$ \\
\hline HEPES buffer, pH 7.4 & $\begin{array}{l}\text { HEPES }(10 \mathrm{mM}) \\
\mathrm{KCl}(160 \mathrm{mM})\end{array}$ \\
\hline sucrose buffer & $\begin{array}{l}\text { HEPES ( } 2 \mathrm{mM}) \\
\text { sucrose }(298 \mathrm{mOsmol} / \mathrm{kg})\end{array}$ \\
\hline
\end{tabular}




\subsubsection{Lipids}

Artificial membrane systems were generated using different lipids. These exhibit various head groups, like phosphocholine (PC) and phosphoethanolamine (PE), which are found in plasma membranes in a high amount. ${ }^{56}$ In order to mimic biological conditions experiments with these lipids were performed. Furthermore lipids with the negative lipid head group phosphatidylserine (PS) were used as the plasma membrane is also enriched with PS (12\%), especially the cytosolic leaflet (cf. chapter 1.4). 37,50

Cholesterol was added in giant unilamellar vesicles (GUVs) as it is known to increase the mechanical stability of membranes. Moreover it can regulate the membrane fluidity. 37,57

1-palmitoyl-2-oleoyl-sn-glycero-3-phosphocholine (POPC), $M=760.1 \mathrm{~g} / \mathrm{mol}$<smiles>CCCCCCCC/C=C\CCCCCCCC(=O)OC[C@H](COP(=O)([O-])OCC[N+](C)(C)C)OC(=O)CCCCCCCCCCCCC</smiles>

1-palmitoyl-2-oleoyl-sn-glycero-3-phosphoethanolamine (POPE), $M=718.0 \mathrm{~g} / \mathrm{mol}$<smiles>CCCCCCCC/C=C\CCCCCCCC(=O)OC[C@@H](COP(=O)([O-])OCC[NH3+])OC(=O)CCCCCCCCCCCC</smiles>

1-palmitoyl-2-oleoyl-sn-glycero-3-phospho-L-serine (POPS), $M=784.0 \mathrm{~g} / \mathrm{mol}$<smiles>CCCCCCCC/C=C\CCCCCCCCCCCC(=O)OC[C@H](O)COP(=O)([O-])OC[C@H](N)C(=O)[O-]</smiles> 
1,2-dioleoyl-sn-glycero-3-phosphocholine (DOPC), $M=786.1 \mathrm{~g} / \mathrm{mol}$

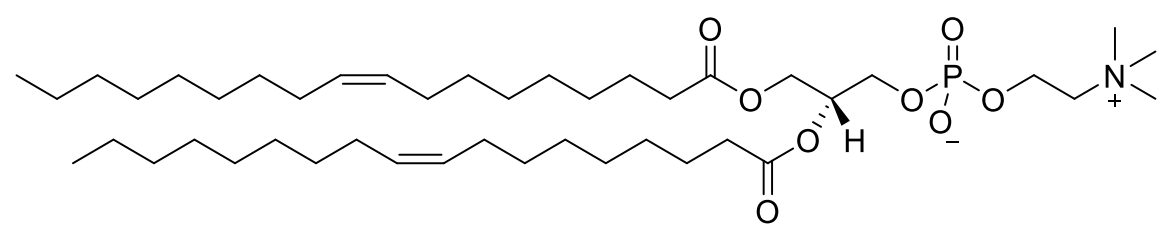

1,2-dioleoyl-sn-glycero-3-phosphoethanolamine (DOPE), $M=744.0 \mathrm{~g} / \mathrm{mol}$

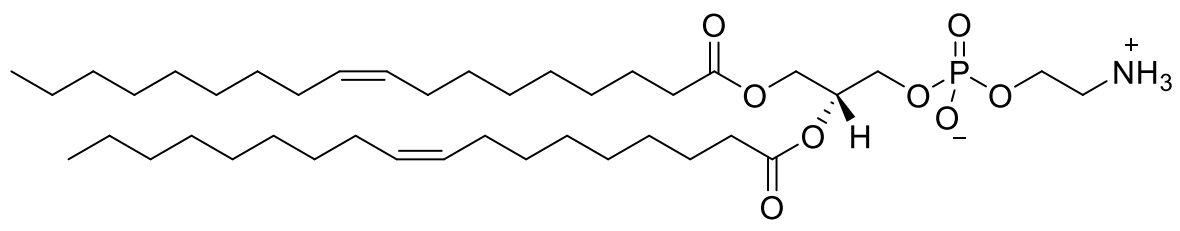

1,2-dioleoyl-sn-glycero-3-phospho-L-serine (DOPS), $M=810.0 \mathrm{~g} / \mathrm{mol}$

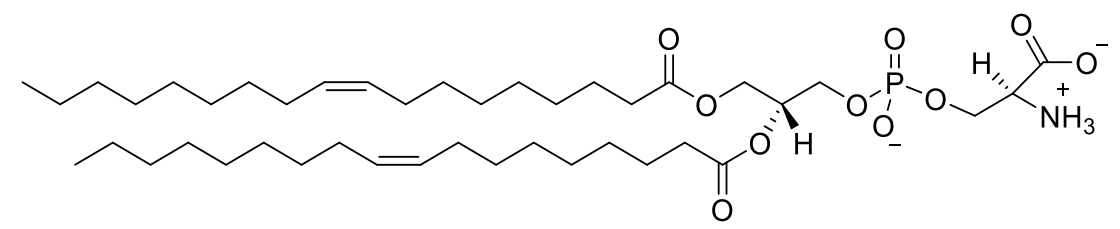

Cholesterol, $M=386.65 \mathrm{~g} / \mathrm{mol}$

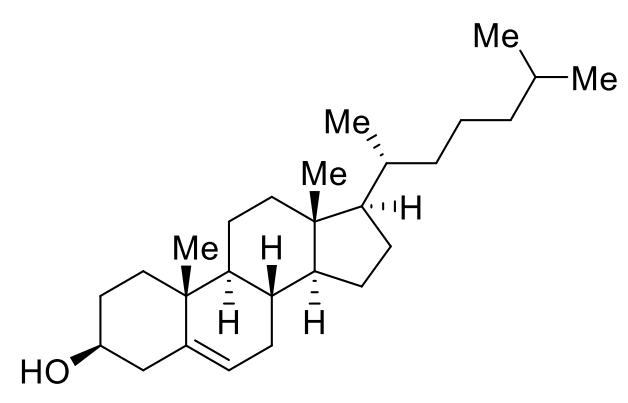

\section{Protein binding lipids}

In this thesis two different lipids were used to bind proteins. L- $\alpha$-phosphatidylinositol-4,5-bisphosphate ( $\left.\mathrm{PIP}_{2}\right)$ is the specific receptor lipid for several proteins and exhibits different functions in biological cells (cf. chapter 1.4). For the experiments in this thesis brain extracted $\mathrm{PIP}_{2}$ was used to bind ENTH. It mainly consists of the fatty acids stearoylic and arachidonoylic acid and has a net charge of -3 to -5 dependent on the $\mathrm{pH}(-4$ at $\mathrm{pH} 3.7)$. 
Moreover 1,2-dioleoyl-sn-glycero-3-phosphoethanolamine- $N$-(cap biotinyl) (cap-biotin-DOPE) was used to bind the protein NeutrAvidin in order to immobilize vesicles via biotin-NeutrAvidin interactions.

L- $\alpha$-phosphatidylinositol-4,5-bisphosphate (PIP 2$), M_{\mathrm{Av}}=1096.4 \mathrm{~g} / \mathrm{mol}$

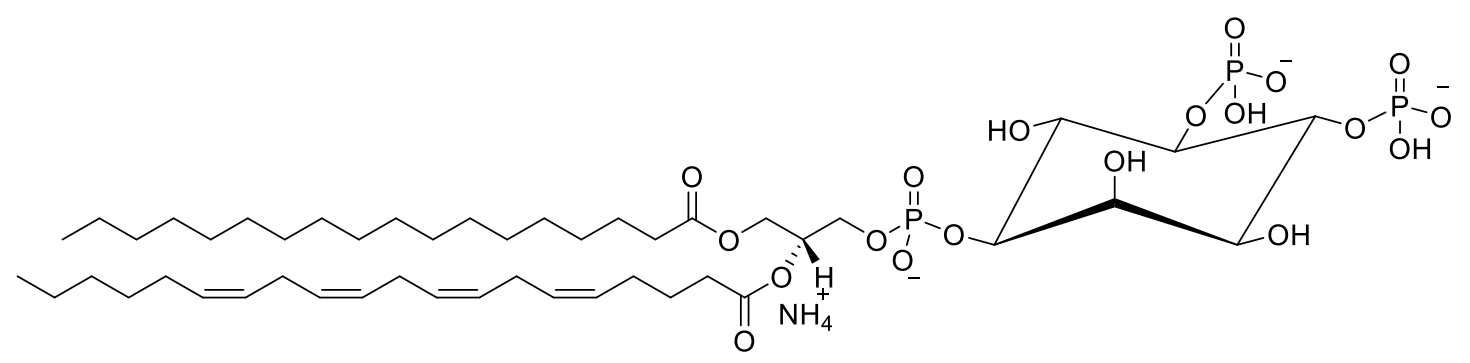

1,2-dioleoyl-sn-glycero-3-phosphoethanolamine- $N$-(cap biotinyl) (cap-biotin-DOPE), $M=992.3 \mathrm{~g} / \mathrm{mol}$

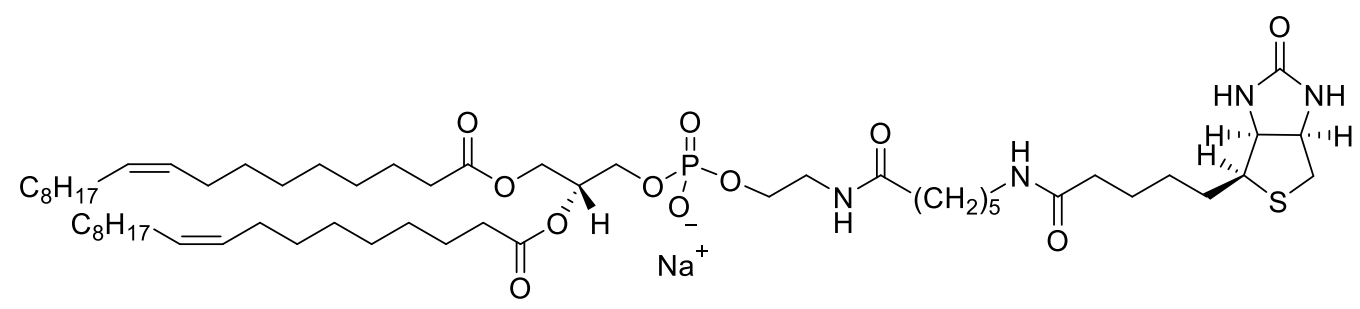

\section{Fluorophores}

To image the artificial membrane models with fluorescence microscopy, the fluorophores Texas $\operatorname{Red}^{\circledR}$ 1,2-dihexadecanoyl-sn-glycero-3-phosphoetanolamine (TxR) and Atto488 1,2-dipalmitoyl-sn-glycero-3-phosphoetanolamine were added to the membranes. 
Texas Red ${ }^{\circledR}$ 1,2-dihexadecanoyl-sn-glycero-3-phosphoetanolamine (TxR), $M=1381.9 \mathrm{~g} / \mathrm{mol}$

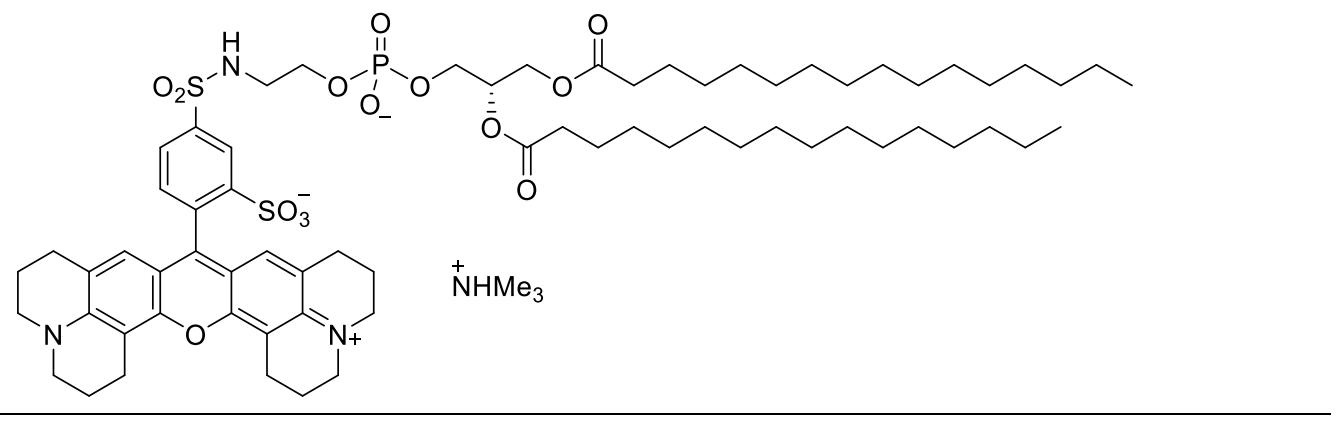

Atto488 1,2-dipalmitoyl-sn-glycero-3-phosphoetanolamine (Atto488-DPPE), $M=1263.6 \mathrm{~g} / \mathrm{mol}$<smiles>CCCCCCCCCCCCCCCCCCCCCC(=O)OCC(COP(=O)([O-])OCCCCCCCCCCC)OC(=O)CCCCCCC(=O)NCCCN(C)C(=O)c1ccccc1-c1c2ccc(=[NH2+])c(S(=O)(=O)O)c-2oc2c(S(=O)(=O)O)c(N)ccc12</smiles>

\subsubsection{Proteins}

\subsubsection{Epsin $N$-terminal homology domain}

The highly conserved epsin $N$-terminal homology (ENTH) domain is an adaptor protein involved in clathrin-mediated endocytosis. It consists of approximately 150 amino acids $(M=16.75 \mathrm{kDa})$ and can be found in many endocytotic proteins. The ENTH domain and its mutant R114A used in this work were isolated and purified by Dr. Benjamin Kroppen and Indrani Mukherjee from the group of Prof. Dr. Michael Meinecke (Department of Cellular Biochemistry, University Medical Center Göttingen). The protein was stored in protein buffer at $-80^{\circ} \mathrm{C}$. Before use the protein was thawed on ice. 


\subsubsection{NeutrAvidin}

NeutrAvidin is a $60 \mathrm{kDa}$ protein and was used for the immobilization of biotinylated giant unilamellar vesicles via a NeutrAvidin-biotin interaction. The protein is the deglycosylated form of the tetrameric glycoprotein avidin. ${ }^{58}$ NeutrAvidin as well as avidin have high binding affinities to biotin, which makes them well-suited for immunological assays and biosensing applications. Deglycosylation does not reduce the biotin binding ability, ${ }^{59}$ but due to the more neutral isoelectrical point ( $\mathrm{pI}=6.3$ ) than avidin $(\mathrm{pI}=10.5)$, less non-specific interactions to surfaces occur. ${ }^{60}$

\subsection{Preparative methods}

\subsubsection{Preparation of unilamellar vesicles}

Vesicles are well suited to investigate protein-membrane interactions. They differ in size and application. The radii vary from $20 \mathrm{~nm}$ (small unilamellar vesicles, SUVs), 100-200 nm (large unilamellar vesicles, LUVs) up to $100 \mu \mathrm{m}$ (giant unilamellar vesicles, GUVs). ${ }^{61-63}$

\section{Small unilamellar vesicles}

For the preparation of small unilamellar vesicles (SUVs) lipid films with $0.4 \mathrm{mg}$ of lipid material were used. These films were obtained by merging different lipid stock solutions (dissolved in chloroform; $c=2-10 \mathrm{mg} / \mathrm{mL}$ ) in a test tube. This enabled to prepare lipid films with defined lipid compositions. After removing the chloroform in a nitrogen flush, the films were dried under vacuum at $30^{\circ} \mathrm{C}$. The films were then stored at $4{ }^{\circ} \mathrm{C}$ until use.

The films were rehydrated with citrate buffer (30 min) and subsequently the test tubes were vortexed three times for $30 \mathrm{~s}$ in a five-minute interval, resulting in the formation of multilamellar vesicles (MLVs). The MLVs were then treated in an ultrasonic bath for 30 min at RT to obtain SUVs. 


\section{Giant unilamellar vesicles}

Giant unilamellar vesicles (GUVs) with a diameter of $\leqq 1 \mu \mathrm{m}$ were prepared by an electro-formation process, first described by Angelova et al.64 A mixture of lipids dissolved in chloroform ( $c=0.5 \mathrm{mg} / \mathrm{mL}$ ) were added on two indium tin oxide (ITO) covered glass slides. The chloroform was removed under reduced pressure for at least $30 \mathrm{~min}$, resulting in a lipid film on top of the ITO. These ITO slides were then assembled to a chamber and sealed with a silicon ring and two Teflon spacers. Afterwards a sucrose solution (298 mOsmol/kg) was filled into the chamber. The connection to the generator was achieved with copper stripes, so each ITO slide was linked to one pole (Figure 3.1). A sinusoidal alternating current voltage of $1.6 \mathrm{~V}$ (peak-to-peak) and $10 \mathrm{~Hz}$ was applied for $3 \mathrm{~h}$, resulting in GUV formation. After collection of the GUVs they were stored at room temperature for a maximum 3 days.

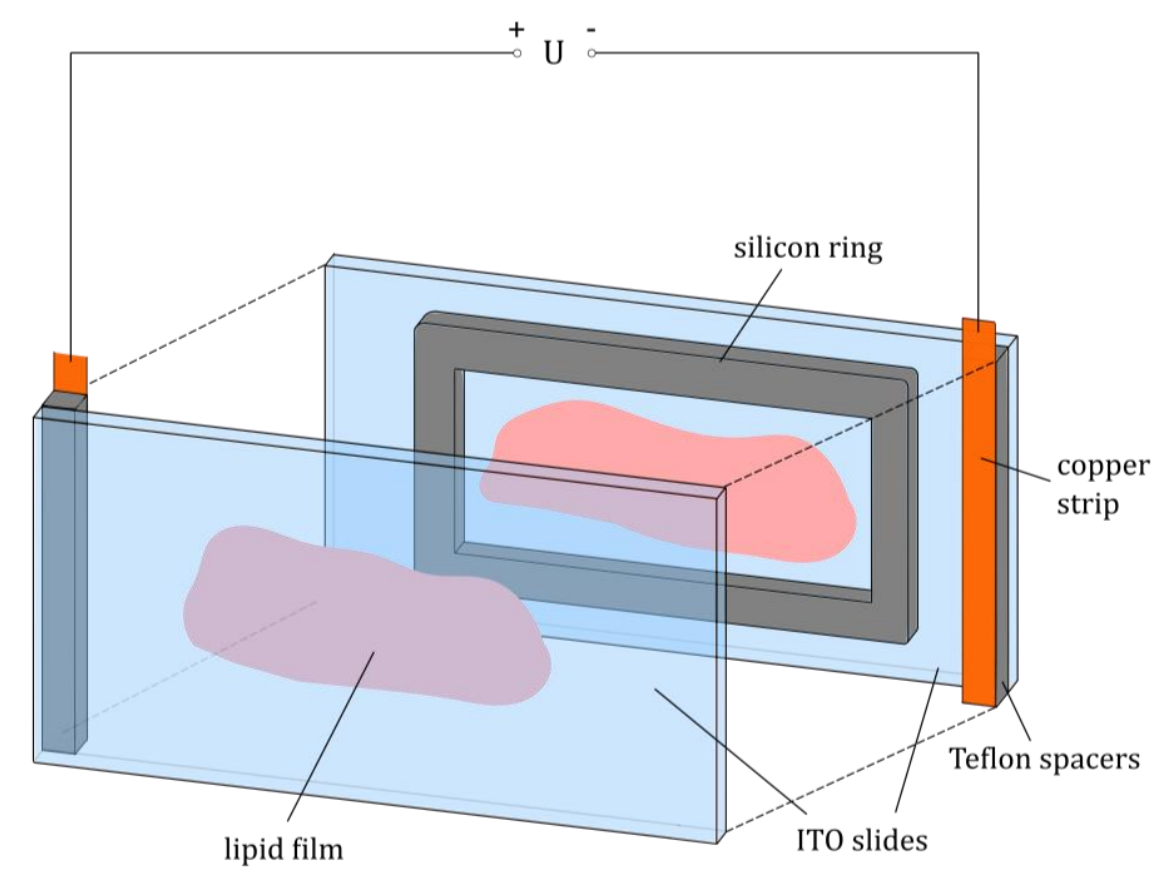

Figure 3.1: Schematic drawing of a GUV electro-formation chamber. On each ITO slide a self-adhesive copper stripe is placed via a Teflon spacer. The Teflon spacers connect the chamber with a voltage source, which results in the formation of GUVs. 


\subsubsection{Substrate surface preparation}

Various experimental methods require different substrates, which vary in material or size. Due to this, surface functionalization strategies can be used to obtain stable membrane models. In this work two different functionalized substrate types were employed to generate supported lipid membranes and adhered giant unilamellar vesicles.

\section{Lipid bilayer and monolayer on silicon dioxide wafers}

Silicon wafers coated with silicon dioxide $\left(\mathrm{SiO}_{2}\right)$ from Silicon Materials, Inc. (PA, USA) were cut into $1.9 \mathrm{~cm}$ x $0.8 \mathrm{~cm}$ rectangles. For reflectometric interference spectroscopy (RIfS) experiments wafers with $5000 \mathrm{~nm} \mathrm{SiO} 2$ layer thickness and for atomic force microscopy (AFM) measurements wafers with $100 \mathrm{~nm} \mathrm{SiO} 2$ were used.

For both techniques the substrates were hydrophilized with an aqueous ammonia hydrogen peroxide solution $\left(\mathrm{H}_{2} \mathrm{O} / \mathrm{NH}_{3}(25 \%) / \mathrm{H}_{2} \mathrm{O}_{2}(30 \%)\right.$ 5:1:1 (v/v/v)) for $30 \mathrm{~min}$ at $70{ }^{\circ} \mathrm{C}$. Hydrophilized substrates were rinsed with ultrapure water and stored in ultrapure water. Before use the substrates were dried in a nitrogen flush and treated with oxygen plasma (30 s, 0.2 mbar, $60 \%$ power). Afterwards, SUVs were spread on the hydrophilic surfaces, resulting in a lipid bilayer.

Lipid monolayers were prepared on hydrophobic silicon wafers. Therefore, the wafers were first cleaned with Hellmanex (15 min) and ultrapure water ( 2 x $15 \mathrm{~min}$ ) in an ultrasonic bath. Then they were treated with oxygen plasma $(30 \mathrm{~s}, 0.2 \mathrm{mbar}, 60 \%$ power) and subsequently incubated with 1,1,1,3,3,3-Hexamethyldisilazane (HMDS). Incubation was performed overnight in a sealed chamber at $120^{\circ} \mathrm{C}$ and under reduced pressure, yielding hydrophobic surfaces (Figure 3.2). 


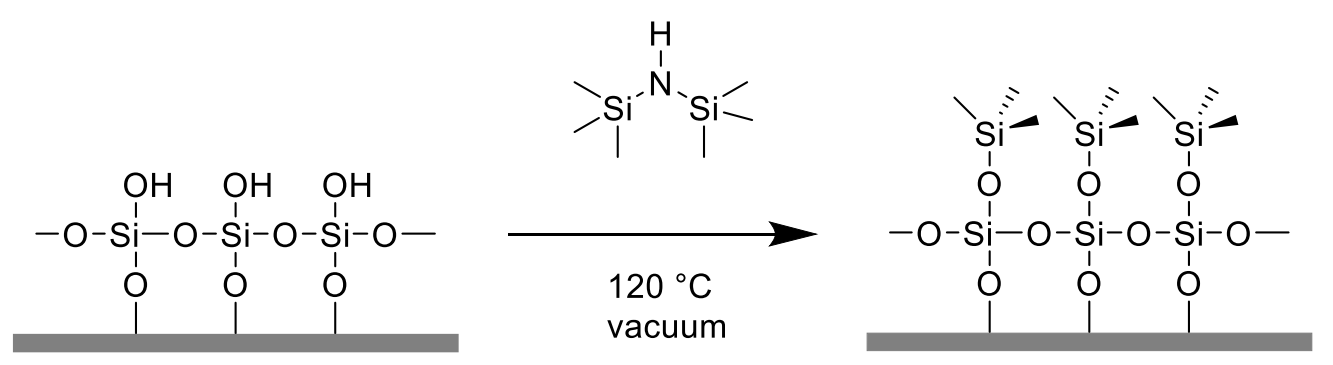

Figure 3.2: Hydrophobic functionalization of silicon dioxide wafers with HMDS.

\section{Silanization and PEGylation to generate adhered GUVS}

First, glass slides had to be functionalized with NeutrAvidin to obtain immobilized biotinylated giant unilamellar vesicles (GUVs). The glass slides (24 x $50 \mathrm{~mm}$, ThermoFisher Scientific Gerhard Menzel, Brunswick, Germany) were cut into $1.0 \mathrm{~cm} \times 1.0 \mathrm{~cm}$ substrates, cleaned with ethanol p.a. and ultrapure water. After treatment with oxygen plasma (30 s, 0.2 mbar, $60 \%$ power) pure 3-glycidyloxi-propyltrimethoxysilane (GOPTS, stored in an argon atmosphere) was added between two substrates and incubated for one hour at $80^{\circ} \mathrm{C}$ in a glass weighing bottle with an argon atmosphere. In that time, a mixture of methoxy- and biotin-functionalized PEGs (1:1, 2:1 and 3:1) was heated in a thermomixer at $85-95^{\circ} \mathrm{C}$ (dependent on the PEG-length) and $1400 \mathrm{rpm}$. The substrates were separated from each other and then rinsed with acetone. Drying in a nitrogen flush and placing the substrates on a preheated aluminum block $\left(80^{\circ} \mathrm{C}\right)$ enabled the addition of $200 \mu \mathrm{L}$ of the molten PEG-mixture between two substrates without solidification. Afterwards, the substrates were placed in the weighing bottle and incubated at $85-95^{\circ} \mathrm{C}$ for $4 \mathrm{~h}$. The substrates were separated, intensely rinsed with ultrapure water and dried in a nitrogen flush. If the molten PEG mixture was still present on the substrates (impure surface), the rinsing step was repeated. Prior to use the substrates were stored under argon at $4{ }^{\circ} \mathrm{C}$. The silanization and PEGylation is illustrated in Figure 3.3. 
silanization: $\quad$ PEGylation:

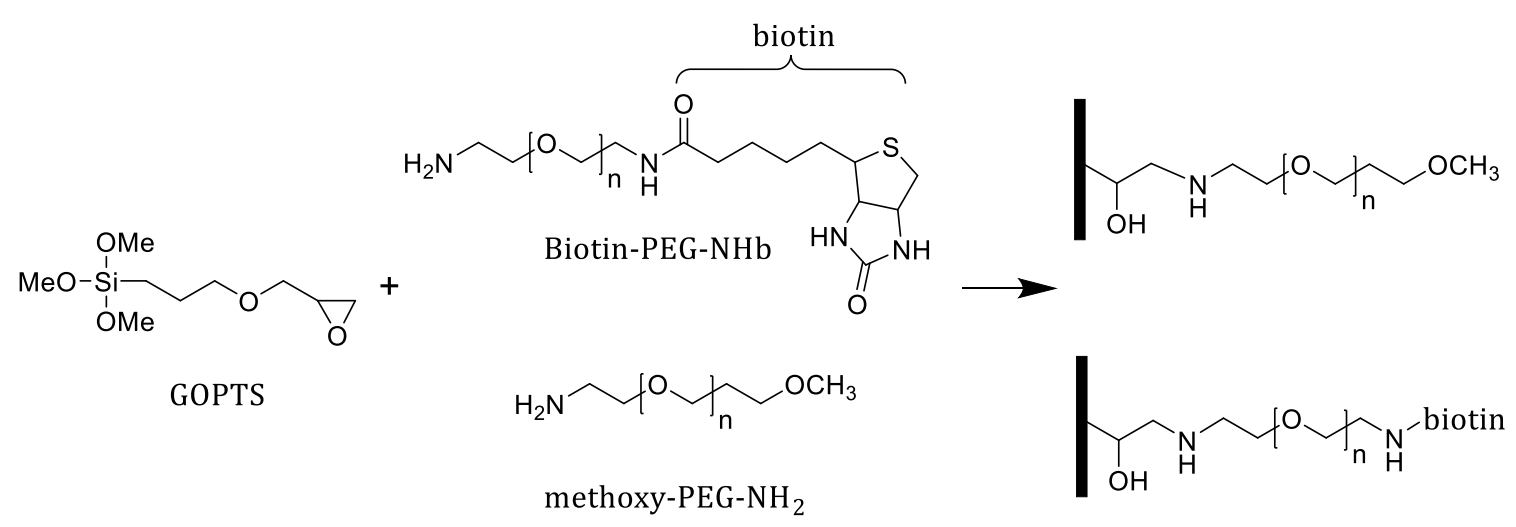

Figure 3.3: Silanization and PEGylation of glass substrates, resulting in methoxy-PEG and biotin-PEG surfaces.

To obtain adhered GUVs, the functionalized substrates were first incubated with NeutrAvidin (50 $\mu \mathrm{g} / \mathrm{mL}$ in PBS) for $30 \mathrm{~min}$. Then the NeutrAvidin-functionalized substrates were rinsed six times with PBS and further six times with sucrose buffer. 10$50 \mu \mathrm{L}$ of a biotin-containing GUV suspension (in sucrose) was added and incubated for $15 \mathrm{~min}$ in a humidity chamber to avoid osmolar changes. Via biotin-NeutrAvidin interactions the GUVs adhered to the surface.

To verify a complete coverage of the substrates with NeutrAvidin, the surface was incubated with DyLight ${ }^{\circledR} 594$ labeled Neutravidin/unlabeled NeutrAvidin in a ratio of 99:1.

\subsection{Biophysical methods}

\subsubsection{UV-Vis spectroscopy}

The determination of ENTH solutions' concentration was done using UV-Vis spectroscopy. This method is based on the absorption of ultraviolet (UV) and visbible (Vis) light. ${ }^{65}$ Proteins with aromatic amino acid residues (Phenylalanine, Trypthophan, Tyrosine) exhibit an absorption maximum at $\lambda=280 \mathrm{~nm}$ due to $\pi \rightarrow \pi^{*}$ transition within 
the amino acids. Measurements of the absorption $A 280$ at this wavelength allowed the calculation of the protein concentration via the Lambert-Beer law:

$$
A_{280}=\varepsilon_{E N T H} \cdot c \cdot d
$$

The extinction coefficient of the ENTH-domain is $25440 \mathrm{M}^{-1} \mathrm{~cm}^{-1}$ and the cuvette thickness is $d=1 \mathrm{~cm}$. The molecular masses and extinction coefficients of ENTH were calculated with the ExPASy ProtParam tool. ${ }^{66}$ The measurements were performed using the NanoDrop ${ }^{\mathrm{TM}}$ 2000c (Thermo Fisher Scientific, Waltham, MA, USA).

\subsubsection{Reflectometric interference spectroscopy}

Reflectometric interference spectroscopy (RIfS) was used to determine the binding of the ENTH domain to lipid bilayers. It is a label-free optical biosensing method, which detects time-resolved changes in optical thicknesses at interfaces between two media with different refractive indices $(n) .67,68$ The method is based on the reflection and transmission of white light at these interfaces ${ }^{69} \mathrm{~A}$ light beam is directed to the interface, where it is partially reflected and refracted. Superimposition of the partial beams leads to a specific interference pattern. Changes of the surface thickness (physical thickness $d$ ) due to e.g. protein adsorption lead to an altered interference spectrum.

The law of Snellius describes the refraction of light on interfaces with the incident angle $\alpha$, the refracting angle $\varphi$ and the different refractive indices $n$ (equation(3.2)).

$$
n_{1} \cdot \sin (\alpha)=n_{2} \cdot \sin (\varphi)
$$

Depending on the refractive indices of the media, the reflection and transmission of the light changes. In Figure 3.4 three possible light pathways are illustrated. 
A

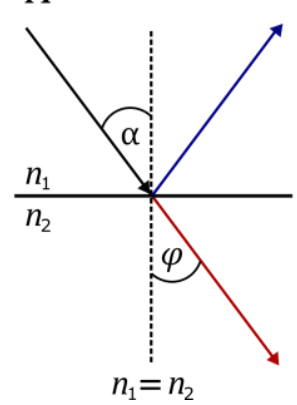

B

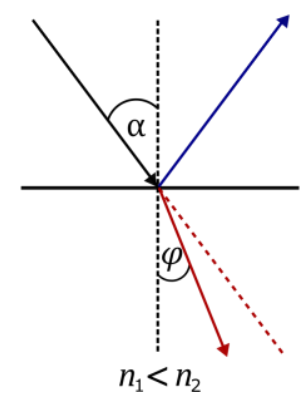

C

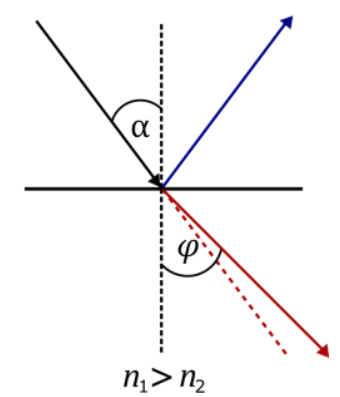

Figure 3.4: Schematic illustration of the SNELLIUS reflaction law. With an incident angle the light is directed to a second medium. Due to the different refractive indices of both media the light can have various light pathways. In case of $n_{1}=n_{2}$ the light is reflected and reflected with the same angles $(\alpha=\varphi)$. When the refractive index of the first medium is higher than the second $\left(n_{1}>n_{2}\right)$, the light is refracted to the vertical $(\alpha>\varphi)$. If the relation of $n_{1}<n_{2}$ occurs, the light is tilted out of the vertical $(\alpha<\varphi)$.

If the refractive indices $n_{1}$ and $n_{2}$ have the same value (Figure 3.4 A), the angles $\alpha$ and $\varphi$ are equal, then the light is reflected and transmitted in the same degree. With $n_{1}<n_{2}$, the light is refracted when it is passing the second medium and the relation $\varphi<\alpha$ is valid (Figure 3.4 B). In the latter case, the transmitted light is tilted out of the vertical due to the higher refractive index of the second medium $\left(n_{1}>n_{2}\right.$, Figure 3.4 C).

In the experimental setup, utilized in this work, the light is directed to a three phase system: An opaque silicon layer followed by a silicon dioxide layer, which is surrounded by an aqueous phase (Figure 3.5). 


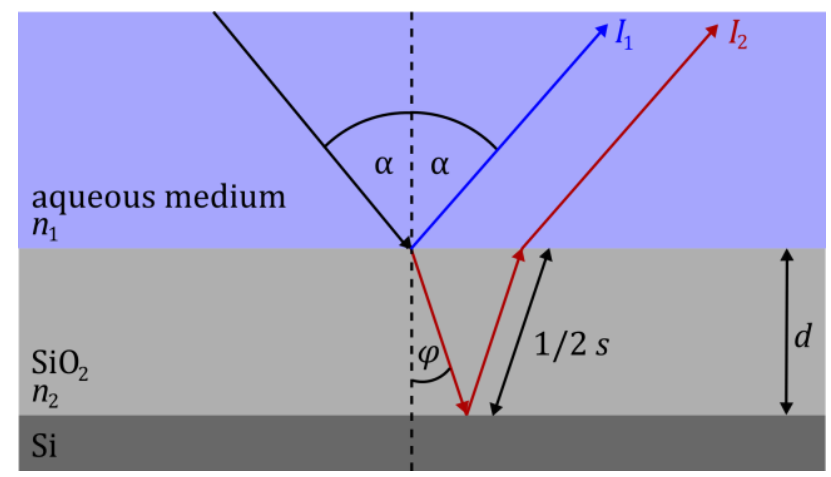

Figure 3.5: Schematic light pathway during a RIfS experiment. The light is directed to a system consisting of three phases with an incident angle $\alpha$. Reaching the silicon dioxide layer with a thickness $d$ after passing the aqueous medium, the light is reflected $\left(I_{1}\right.$, blue) and refracted $\left(I_{2}\right.$, red) to the vertical at an angle $\varphi$ due to different refractive indices $\left(n_{\mathrm{i}}\right)$. As the silicon layer is intransparent the light is completely reflected, resulting in an additional traveling distance $s$ for $I_{2}$.

With the incident angle $\alpha$ the light passes the aqueous medium $\left(n_{1}=1.33\right.$ at $\left.20^{\circ} \mathrm{C}\right) .{ }^{70}$ When it is hitting the silicon dioxide layer $\left(n_{2}=1.45\right)^{71}$ the light is refracted towards the normal at the angle $\varphi$. The opaque silicon layer reflects the light beam, which is then refracted once more at the interface of media with $n_{1}$ and $n_{2}$. The result is a phase shift $\Delta \varphi$ due to the longer traveling distance $(s)$ of the refracted light $\left(I_{2}\right)$ compared to the reflected part $\left(I_{1}\right)$. The distance can be calculated with equation (3.3).

$$
s=\frac{2 d}{\cos (\varphi)}
$$

The optical distance is dependent on the refractive index of the second medium $n_{2}$ (equation (3.4)), which leads to equation (3.5).

$$
\begin{gathered}
s_{O T}=n_{2} \cdot s \\
s_{O T}=\frac{2 n_{2} d}{\cos (\varphi)}
\end{gathered}
$$

The refraction of the light causes a phase shift, resulting in constructive and destructive interference. When constructive interference arises the $\Delta \varphi$ is a multiple of the wavelength, described by equation (3.6). 


$$
\Delta \varphi(\lambda)=\frac{2 \pi \cdot O T}{\lambda} \cos (\varphi)
$$

The optical thickness changes by adsorption of molecules on the surface as it is the product of $n$ and $d$ (equation (3.7)). Since the refractive indices of silicon dioxide and lipid or protein layers are similar, ${ }^{72}$ it hence enables to measure the molecule adsorption by detection of the optical thickness changes.

$$
O T=n \cdot d
$$

In case of a perpendicular incident light causing $\alpha=\varphi=0^{\circ}$, equation (3.7) simplifies to equation (3.8). This allows to monitor the optical thickness change via phase shift detection.

$$
\Delta \varphi(\lambda)=\frac{2 \pi \cdot O T}{\lambda}
$$

\section{Evaluation of the data}

A reflectivity spectrum was determined by the program Spectra Suite (Ocean Optics Inc., Dunedin, FL, USA) with the reference $\left(I_{\mathrm{r}}\right)$ and the dark $\left(I_{\mathrm{d}}\right)$ spectrum according to equation (3.9). The reference spectrum is recorded at nearly complete reflectivity against a silver-coated surface. Measurement of the light intensity without illumination yielded the dark spectrum.

$$
R(\lambda)=\frac{I_{\mathrm{m}}-I_{\mathrm{d}}}{I_{\mathrm{r}}-I_{\mathrm{d}}}
$$

$I_{\mathrm{m}}$ is the measured intensity spectrum. The reflectivity is described with the Fresnel reflectivity coefficients $r_{1}$ and $r_{2}$ in equation (3.10).

$$
R(\varphi)=\frac{r_{1}^{2}+2 r_{1} r_{2} \cos (2 \varphi)+r_{2}^{2}}{1+2 r_{1} r_{2} \cos (2 \varphi)+{r_{1}}^{2}{r_{2}}^{2}}
$$

Thereby, the Fresnel reflectivity coefficients are defined as: 


$$
r_{1}=\frac{n_{1}-n_{2}}{n_{1}+n_{2}} \text { and } r_{2}=\frac{n_{2}-n_{3}}{n_{2}+n_{3}}
$$

Combination of equation (3.10) and (3.11) leads to the reflectivity, which is dependent on the wavelength and the optical thickness OT. This in turn enables the timeresolved tracking of OT (equation (3.12)).

$$
R(\lambda, O T)=\frac{r_{1}^{2}+2 r_{1} r_{2} \cos \left(\frac{4 \pi}{\lambda} O T\right)+r_{2}^{2}}{1+2 r_{1} r_{2} \cos \left(\frac{4 \pi}{\lambda} O T\right)+r_{1}{ }^{2} r_{2}{ }^{2}}
$$

\section{Experimental procedure}

The hydrophilic or hydrophobic functionalized silicon wafers (cf. chapter 3.2.2) were implemented into a RIfS chamber, consisting of an aluminum bottom, an acrylic glass cap and a rubber ring to seal the chamber (Figure 3.6).

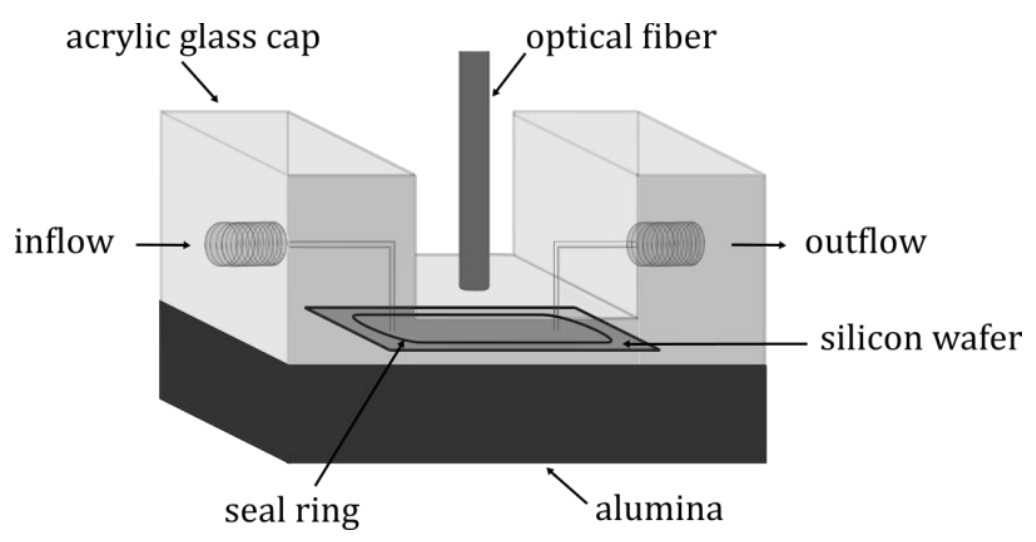

Figure 3.6: Schematic illustration of a RIfS chamber. A silicon wafer is mounted between an acrylic glass cap and an alumina plate. In and outflow of liquids is realized by inlets in the acrylic glass cap. Then the optical fiber is placed in the indentation of the acrylic glass cap.

The flow in and out of the chamber was conducted via inlets inside the acrylic glass cap, which enabled a controlled addition of liquids. A fanned optical fiber was orthogonally mounted to the wafers. This fanned fiber is made of one light-collecting fiber, which is surrounded by six emitting fibers connected to a tungsten halogen lamp $(\lambda=350-950 \mathrm{~nm})$. The reflected and refracted light of the $1 \mathrm{~mm}^{2}$ lighted area is detected by a UV/Vis-silicon-CCD-detector (2048 Pixel). 
Every two seconds a reflectivity spectrum was recorded with the software Spectra Suite and then tracked with a MATLAB script using equation (3.12) written by Dr. I. Mey (Georg-August-University Göttingen).

For the experiment, the system was first rinsed with citrate buffer, which was defined as the baseline for the determination of the optical thickness changes. Then SUVs were added, leading to an increase of the optical thickness. This increase indicates the spreading and formation of a lipid bilayer (hydrophilic) or monolayer (hydrophobic). After a plateau was reached, PBS was rinsed through the chambers to remove nonspread SUVs. Then, BSA (1 mg/mL) was added for surface passivation, preventing possible unspecific interactions of ENTH with defects in the membranes. Before protein addition, the system was again rinsed with PBS, the same buffer in which the ENTH domain is solved. For the investigation of the influence of $\mathrm{PIP}_{2}$ on the ENTHmembrane interaction, measurements with a single protein addition with a concentration of $1 \mu \mathrm{M}$ were implemented. Isotherms with gradual protein additions $(c($ ENTH $)=0.1-5 \mu \mathrm{M})$ ) were performed to detect the influence of the charged lipid Phosphatidylserine (PS) on the ENTH binding affinity. Due to the interaction of the membrane/surface and the added protein, an increase of the optical thickness was monitored. When a plateau was reached, PBS was rinsed through the system to detect the protein dissociation process or more protein was added to obtain a Langmuir adsorption isotherm.

\subsubsection{Fluorescence microscopy}

Fluorescence microscopic techniques were used to image solid-supported membranes and adhered GUVs. This non-invasive method bases on the physical phenomenon of fluorescence. After the absorption of light, fluorophores emit the light with a longer wavelength (stokes shift). Separation with filters and mirrors allows the collection of the emitted light, excluding illumination light. There are different types of fluorescence microscopy setups, which enable to image labeled biological structures in high resolution. In the next chapters two types of microscopes are described in more detail. 


\subsubsection{Confocal laser scanning microscope}

The confocal laser scanning microscope (CLSM) uses a single laser beam, which is focused onto a specimen. As the emitted light is filtered through a pinhole rejecting outof-focus light, the sample is scanned point-wise. Therefore, higher axial resolutions can be reached compared to an epifluorescence microscope..$^{73}$ Due to the illumination of a small focal volume of the sample moreover the bleaching of fluorophores is reduced. In Figure 3.7 an illustration of the setup and the light path of a CLSM is shown.

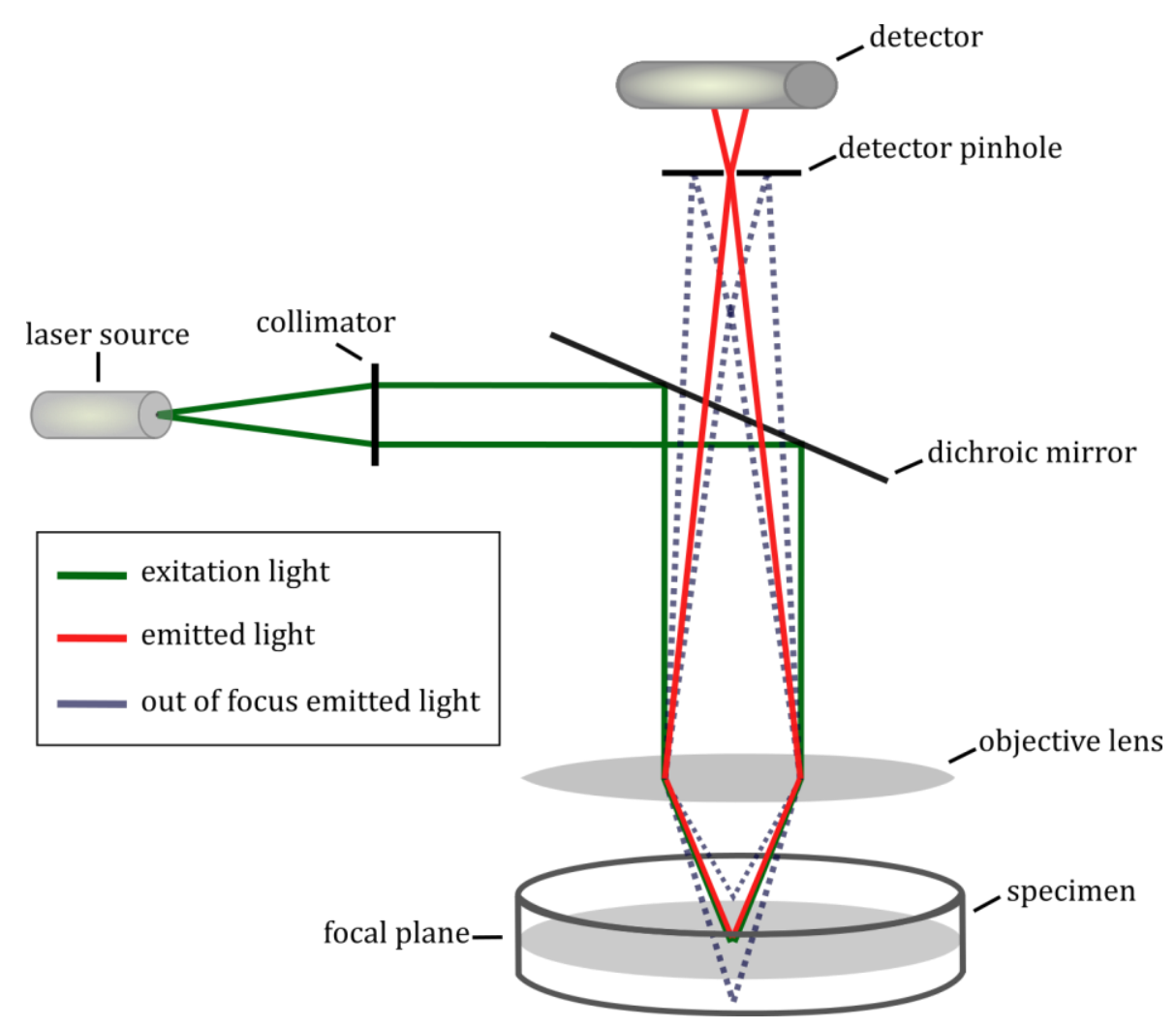

Figure 3.7: Schematic illustration of a confocal laser scanning microscope setup. The fluorophore of the specimen is excited by a laser. The emitted light (red) passes the dichroic mirror and is focused to the detector via a confocal pinhole, which enables to reject out-of-focus light (blue dotted lines).

The resolution of a CLSM using very small pinholes can be determined by the full width at half maximum (FWHM) of the point spread function according to Abbes law, enabling lateral and axial resolutions in the range of 200-250 and 570-950 $\mathrm{nm}^{74}$ when the specimen was exited with visible light (equations (3.13) and (3.14)). 


$$
\begin{gathered}
d_{\text {FWHM,lateral }}=\frac{0.37 \overline{\bar{\lambda}}}{N A} \\
d_{\text {FWHM,axial }}=\frac{0.67 \overline{\bar{\lambda}}}{n-\sqrt{n^{2}-N A^{2}}}
\end{gathered}
$$

$\bar{\lambda}$ is described by the wavelength of the emitted $\left(\lambda_{\mathrm{em}}\right)$ and the exited light $\left(\lambda_{\mathrm{ex}}\right)$ with $\sqrt{\lambda_{\mathrm{em}} \cdot \lambda_{\mathrm{ex}}}, n$ is the refractive index of the imaged medium and $N A$ the numerical aperture of the objective.

The CLSM setup was used to image solid-supported membranes as well as to determine diffusion coefficients of these membranes via fluorescence recovery after photobleaching experiments.

\section{Fluorescence recovery after photobleaching}

Fluorescence recovery after photobleaching (FRAP) is a method to determine the lateral mobility of labeled molecules. First, the fluorescence intensity of a defined circular region of interest (ROI, Figure 3.8, red circle) of the sample is measured. Subsequently, fluorophores in this region are irreversibly bleached by a laser beam. As far as the lipids are mobile, the fluorescence intennsity in the ROI increases after a time due to diffusion of unbleached molecule into the ROI and diffusion of bleached molecules out of the ROI. In Figure 3.8 a typical FRAP experiment is illustrated. 


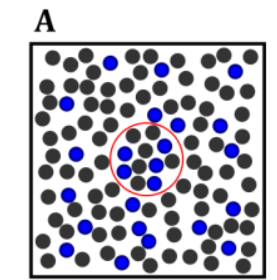

$t<t_{0}$

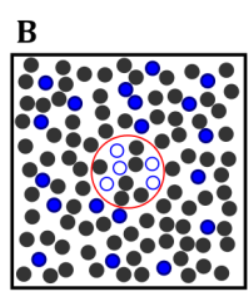

$t_{0}$

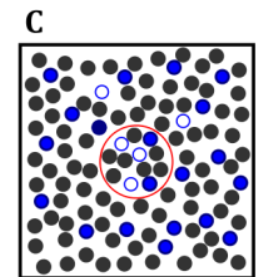

$t_{0}<t<t_{\infty}$

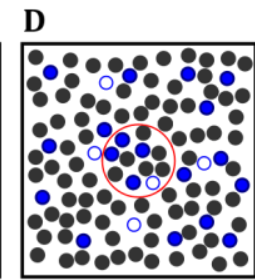

$t_{\infty}$

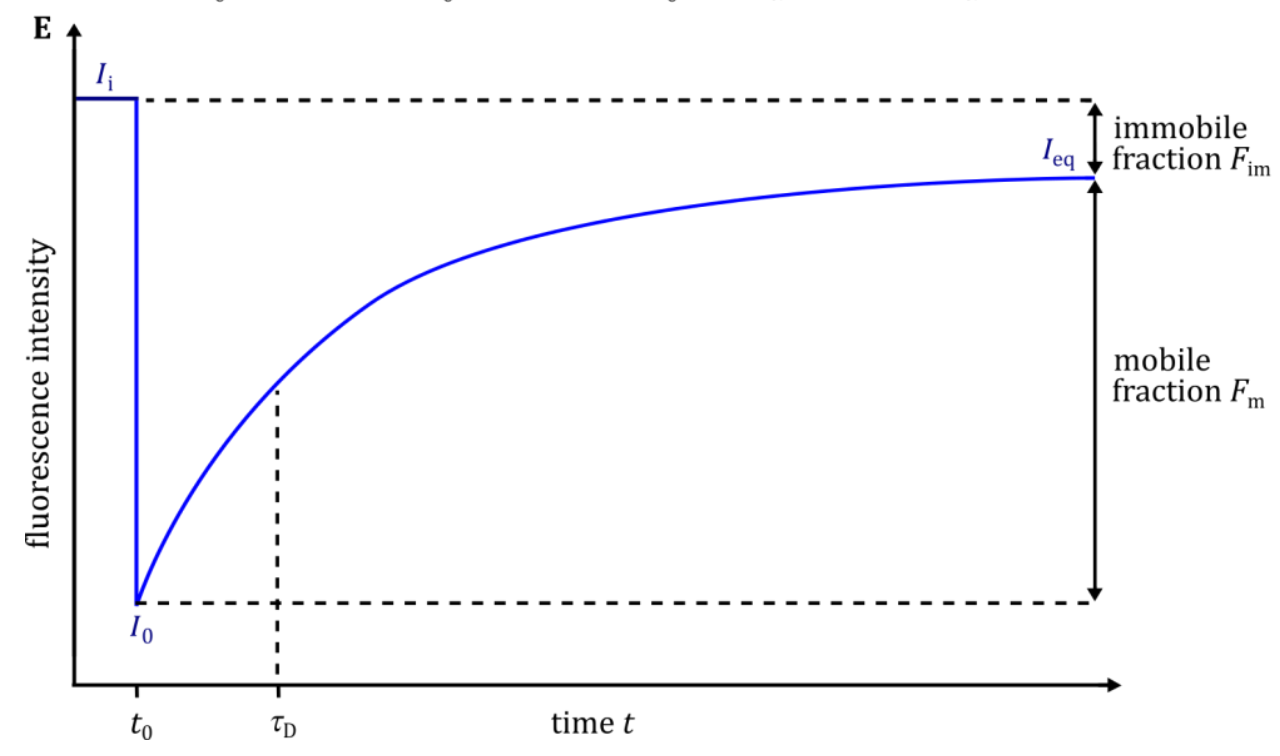

- bleached fluorophore

$\circ$ unlabeled lipids

Figure 3.8: Schematic illustration of a FRAP experiment. (A) At the beginning the fluorescence intensity $I_{\mathrm{i}}$ in a circular region of interest (ROI, red circle) is detected. The lipids are distributed homogenously (B) Bleaching of labeled lipids in the ROI leads to the decrease of the fluorescence intensity to $I_{\mathrm{o}}$ in corresponding time trace (E). (C) Due to lateral mobility labeled lipids diffuse out of the ROI and nonlabeled lipids into the ROI, which increases the fluorescence intensity in the ROI. (D) At the end the a new equilibrium of the fluorescence intensity $I_{\text {eq }}$ is reached. ${ }^{75}$

The graph in Figure 3.8 E shows an exponential increase of the intensity after bleaching of labeled lipids in the ROI. The time dependent fluorescence intensity $I(t)$ can be described with the fluorescence intensity directly after the bleaching $I_{0}$, the intensity at the new equilibrium $I_{\text {eq, }}$, the time $t$ and the time constant of the fluorescence recovery $t_{\mathrm{F}}$ (equation (3.15)). ${ }^{76,77}$

$$
I(t)=I_{\mathrm{eq}}-\left(I_{\mathrm{eq}}-I_{0}\right) \cdot \exp \left(\frac{-t}{t_{\mathrm{F}}}\right)
$$

The time constant is related to the characteristic diffusion time $\tau_{\text {D }}$ by equation (3.16). 


$$
\tau_{\mathrm{D}}=\ln (0.5) \cdot\left(-t_{\mathrm{F}}\right)
$$

With the radius of the ROI $(r)$ and $\tau_{\mathrm{D}}$ it is possible to calculate the diffusion coefficient $D$ using equation (3.17).

$$
D=\frac{r^{2}}{4 \tau_{\mathrm{D}}}
$$

Dependent on the interactions of the substrate and the membrane, a certain amount of the lipids remains immobile. The mobile fraction $F_{\mathrm{m}}$ can be determined with equation (3.18).

$$
F_{\mathrm{m}}=\frac{I_{\mathrm{eq}}-I_{0}}{I_{\mathrm{i}}-I_{0}}
$$

describes the initial fluorescence intensity before bleaching. The diffusion coefficients and the mobile fraction were determined with a MatLab Script written by Jönsson et al. using a Hankel transformation. ${ }^{78}$

\section{Experimental procedure}

The experiments were performed with an upright microscope (LSM 880 Examiner, Carl Zeiss, Jena, GER) equipped with a water immersion objective (WPlan-Apochromat 63x, NA 1.0, Carl Zeiss, Jena, GER). SUVs were prepared as described in chapter 3.2.1. To enable the visualization of the membranes, a fluorophore was incorporated. In this thesis the lipid bound dye TxR was used. TxR was excited at $561 \mathrm{~nm}$ and detected at 570-680 $\mathrm{nm}$. After functionalization of the substrates (cf. chapter 3.2.2) they were implemented into Teflon chambers. Depending on the functionalization, spreading of SUVs for $1 \mathrm{~h}$ resulted in mono- (hydrophobic) or bilayers (hydrophilic). Nonspread vesicles were removed by rinsing with citrate buffer ( $3 \mathrm{x})$ and PBS (3x). Subsequently, imaging and FRAP experiments were performed. 


\subsubsection{Spinning Disc}

Compared to a conventional CLSM a spinning disc confocal laser microscopy (SDCLM) has the advantage that imaging works much faster due to multiple beam illumination. This is realized with two synchronically rotating discs. Excitation light first passes the micro lens array disc and is then focused onto the sample by a second Nipkow disc with multiple (about 1000-20000) spirally arranged pinholes (50 $\mu \mathrm{m})$. The SDCLM setup was used to image z-stacks of biotinylated vesicles adhered to a NeutrAvidin functionalized surface. In Figure 3.9 the setup of a SDCLM is illustrated.

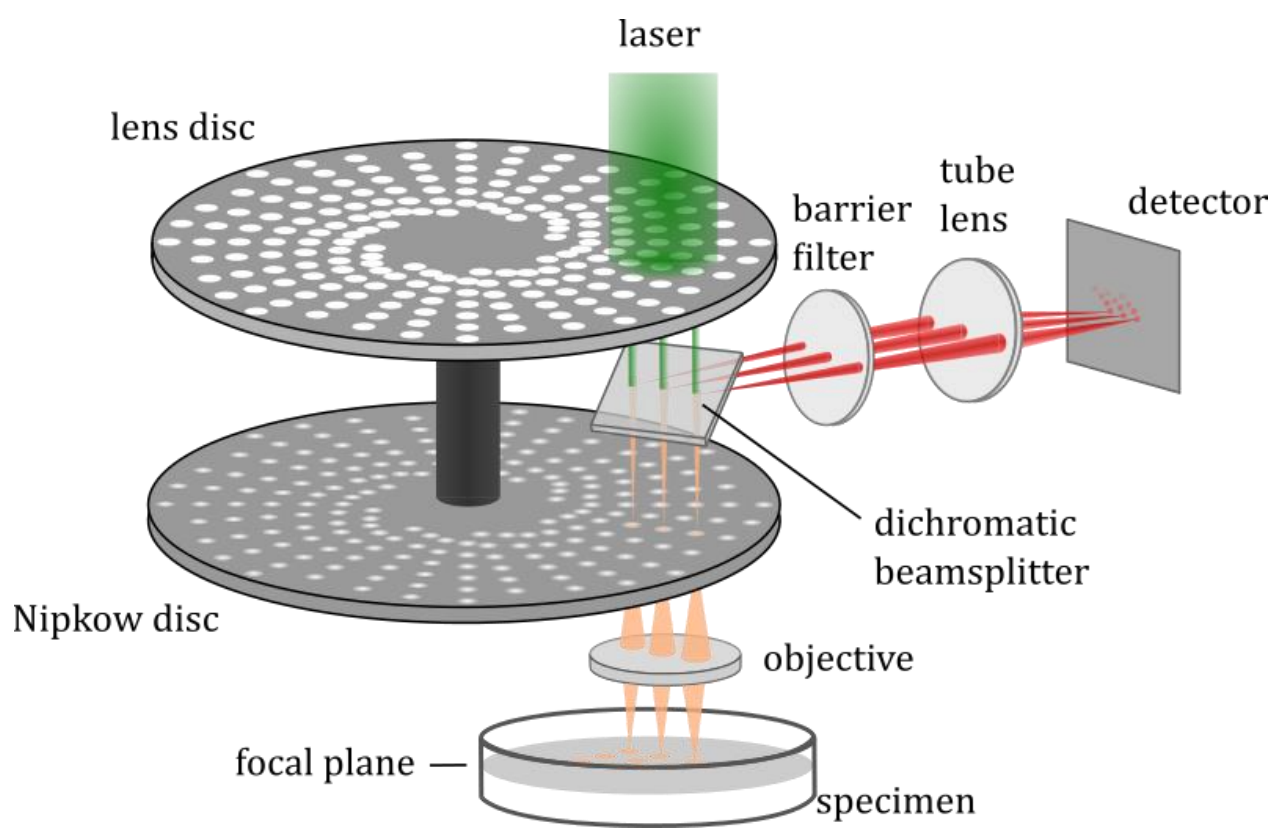

Figure 3.9: Setup of a spinning disc. Laser light is directed to a specimen by the micro lens disc passing through pinholes of a second disc (Nipkow disc). The emitted light is focused to the beamsplitter by the objective lens through the Nipkow disc pinholes, where the light is directed to the detector, passing a barrier filter and a tube lens. The figure was modified according to Gleisner. ${ }^{79}$

\section{Experimental procedure}

The measurements were performed using an upright SDCLM (spinning disc: Yokogawa CSU-X, Rota Yokogaa GmbH \& Co. KG, Wehr, stand: custom made Olympus Deutschland GmbH, Hamburg) equipped with an iXON 897Ultra camera (Andor Technology Ltd., Belfast, UK) and a water immersion objective (CFI Plan 100XW 100x, NA 1.1, Nikon, Düsseldorf). The dye Atto488-DPPE, incorporated in the GUVs, was excited at $488 \mathrm{~nm}$. A LP496 ET filter was used to detect the emission of the fluorophore. 
Adhered GUVs were prepared as described in chapter 3.2.2. The use of a humidity chamber avoided the evaporation of the buffer and consequently ensured iso-osmolar conditions during the experimental time. A series of z-stacks of the adhered GUVs were recorded with 5-13\% laser power and with an exposure time of 20 ms. The distance between the slices was adjusted to $150-250 \mathrm{~nm}$. The radii of the GUVs and the contact areas of the GUVs with the surface were determined with ImageJ.

\subsubsection{Atomic force microscopy}

Binning et al. first introduced the atomic force microscopy (AFM) technique in 1985.80 It enabled scanning of non-conductive probes under physiological conditions with a high lateral $(1.0 \mathrm{~nm})$ and axial $(0.1 \mathrm{~nm})$ resolution. ${ }^{81}$ Hence it is well suitable for the analysis of biological systems, like e.g. receptor-ligand interactions. ${ }^{82}$ In this work AFM was used to analyze surface topographies of solid-supported membranes and ENTH clusters.

\section{Principle of an AFM}

A cantilever with a sharp tip is moved over a sample by x-y piezo actuators. Furthermore, a z-piezo actuator can approach the cantilever towards the sample until attractive or repulsive interactions of the sample and the cantilever lead to the deflection of it. The deflection is detected by a laser beam, which is reflected by the cantilever surface as well as by a mirror and then directed to a four quadrant diode. Lateral or vertical deflection results in the shift of the laser beam on the diode. Hence the deflection of the cantilever against the sample position allows to get a topographic image of the sample surface. The setup of an AFM is illustrated in Figure 3.10. 


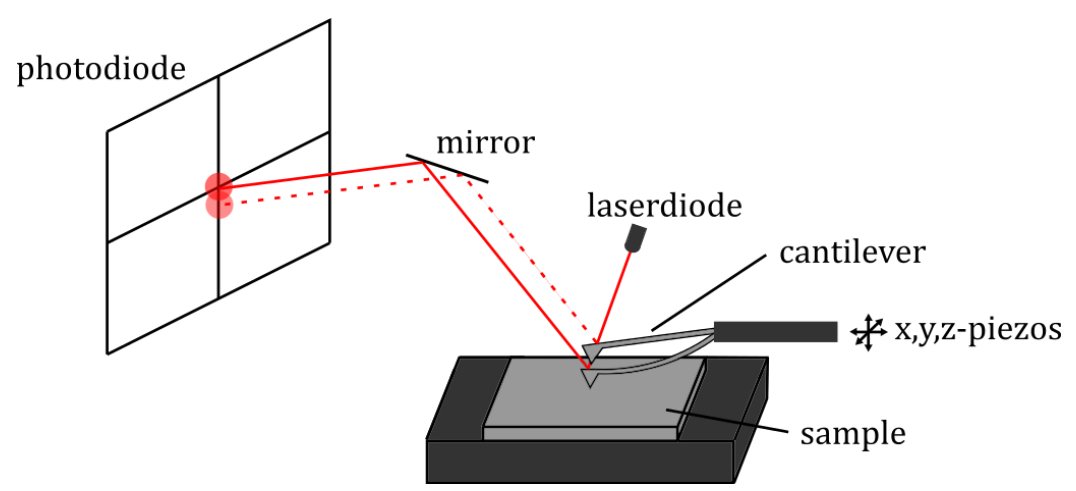

Figure 3.10: Schematic illustration of an AFM setup. The piezos move the cantilever on the surface. A light beam, which is directed to the cantilever, is reflected and directed to the four quadrant photodiode, detecting the deflection of the cantilever.

In AFM different imaging modes can be applied. In this work the contact mode was used, where the tip is in close contact to the sample. The contact mode can be realized in constant height or constant force. In case of constant force a topographic image can be illustrated due to the piezo element regulation. This mode appeared to be best suited for imaging ENTH clusters on solid-supported membranes.

Besides topographical information, AFM is utilized for the determination of mechanical properties of samples by force-distance curves. This method is often used to measure the elastic characteristics of biological systems. ${ }^{83}$ Thereby, the force can be obtained by the vertical cantilever defection $Z_{\mathrm{c}}$ and the spring constant of the cantilever $(k)$ by Hooke's law (equation (3.19)). ${ }^{84}$

$$
F=k \cdot Z_{\mathrm{c}}
$$

For force-distance curves the position of the piezo $Z_{\mathrm{p}}$ and the cantilever deflection $Z_{\mathrm{c}}$ are converted into force $F$ and distance $D$ (Figure $3.11 \mathrm{~B}$ ). Applying a fit to the compliance region yielded the slope, which is equal to the conversion factor of the cantilever defection and the detector signal. The tip-sample distance can be obtained by equation (3.20). ${ }^{85}$

$$
D=Z_{\mathrm{p}}+Z_{\mathrm{c}}
$$


Force-distance curves were detected at single sample positions and predefined forces of 6-8 $\mathrm{nN}$ to obtain breakthrough forces which provides the membrane thicknesses as they are correlated (Figure 3.11).

A

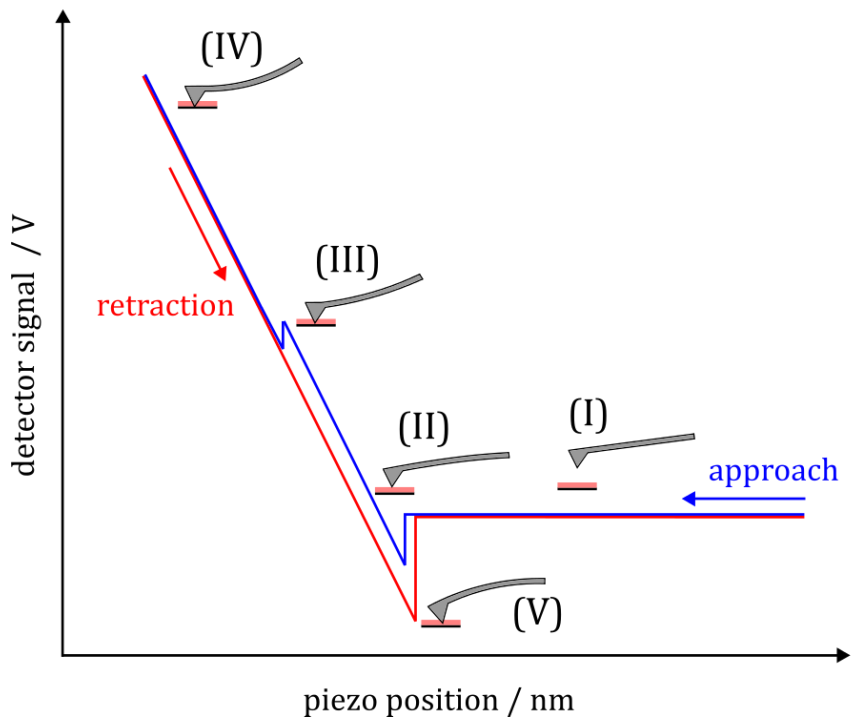

B

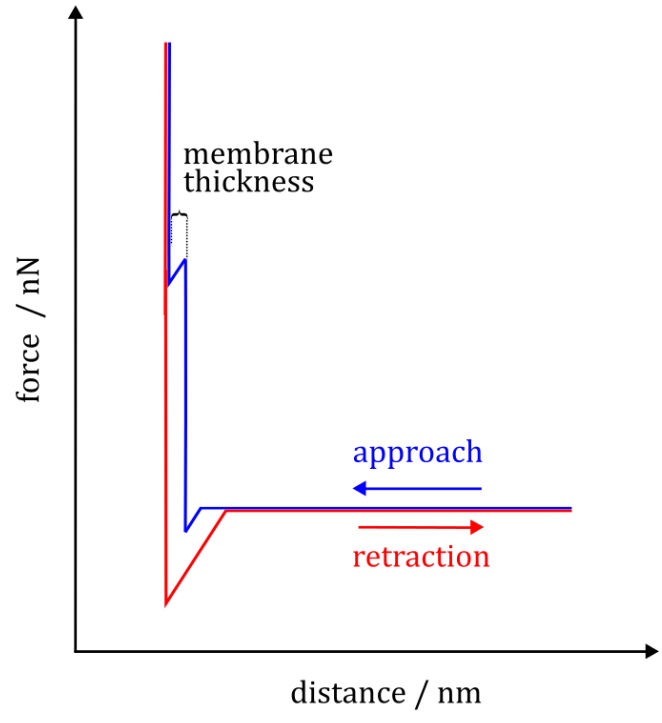

Figure 3.11: Schematic illustration of a signal versus piezo position (A) and a force-distance curve (B). During approach (blue) no interactions of cantilever and sample occur (I). Due to attractive forces the cantilever gets in contact with the sample and the measured force suddenly decreases (II). Further approach leads to increase of the force (IV). At high predefined forces the cantilever can break through the lipid bilayer resulting in a second drop of the force (III). Until the retraction energy exceeds the adhesion energy the cantilever stays in contact to the surface during retraction (red, V). At the end the baseline is reached again as no contact of cantilever and sample is present.

By the approach of the cantilever the force suddenly decreases due to attractive electrostatic or van-der-Waals interactions. As the cantilever is in contact further approach results in the increase of $F$ until a predefined value. At high predefined forces a small drop in the approach force-distance curve can appear (cantilever tip breaks through the bilayer), enabling the determination of the membrane thickness. In the force-distance plot the membrane thickness is the distance between the decrease of $F$ and the point where $F$ begins to rise again (cf. Figure $3.11 \mathrm{~B}$ ). ${ }^{86}$ Then retraction of the cantilever leads to the decrease of $F$. An even negative force value can be reached when adhesion causes cantilever bending. With further retraction the cantilever loses the contact as the retraction force exceeds the adhesion force. 


\section{Experimental setup}

For the supported lipid bilayers (SLBs) previously described FRAP and CLSM experiments (cf. chapter 3.3.3.1) were done to verify the mobility of the membranes. Measurements were performed using a JPK Nanowizard 4 (JPK Instruments, Berlin, GER). After fixing the cantilever to a glass holder and implementing both into the AFM head, the laser was directed to the tip of the cantilever to get a maximal signal. The exact spring constant of the cantilever was determined by measuring the thermal noise spectrum. 87

As all settings were adjusted, micrographs of SLBs were taken to analyze the surface topography before and after protein adsorption. SLBs were prepared as described in chapter 3.2.2. Measurements were done in contact mode using BL-AC40TS-C2 cantilevers (BioLever mini, $f=85.4-139.1 \mathrm{kHz}, k=0.03-0.12 \mathrm{~N} / \mathrm{m}$, Olympus). First of all break-through experiments were performed to measure the membrane thickness as it can be derived from the break-through force. Besides FRAP experiments, this ensured that a bilayer with a thickness of about $4 \mathrm{~nm}$ was formed, which is typical for a bilayer. ${ }^{88} \mathrm{Next}$, an area of $10 \times 10 \mu \mathrm{m}^{2}$ of the SLBs was imaged. After incubation with $1 \mu \mathrm{M}$ ENTH or ENTH R114A mutant for $2 \mathrm{~h}$ at RT, the surface was scanned again. Roughnesses (root-mean-square, rms) were determined with the integrated JPK Data Processing software.

The protein height and occupancy were analyzed using a MatLab script written by Dr. Ingo Mey (Georg-August Universität Göttingen). To detect the membrane and the protein a threshold was set. Protein adsorption was identified by a 2D peak detection function, which marked the local maxima. A histogram out of the maxima was created and fitting a normal distribution yielded the protein height. 


\subsubsection{Langmuir-Blodgett trough}

Monolayers have the advantage of a homogenous lipid distribution without the feasible asymmetry between two leaflets of bilayers. One possibility to generate monolayers without the necessity of a solid support is the Langmuir-Blodgett trough. This technique thus enables to prevent interactions of lipids with substrate surfaces. Besides the determination of lipid concentrations and properties, also the compressibility of monolayers can be analyzed. Furthermore, single or multiple monolayers on solid supports (so called Langmuir films) can be generated with this technique.

Studies with spread lipids on water already were done by Agnes Pockels in 1882.89 However, the technique as it is known and used today was extended and improved by Irving Langmuir and Katherine Blodgett in 1917 and 1934.

The Langmuir-Blodgett trough or film balance consists of a temperature controlled Teflon trough, Teflon barriers and a microbalance system with a Wilhelmy plate. ${ }^{90}$ These barriers can be moved by a control system to change the trough area and enable the compression or expansion of spread films (Figure 3.12). 


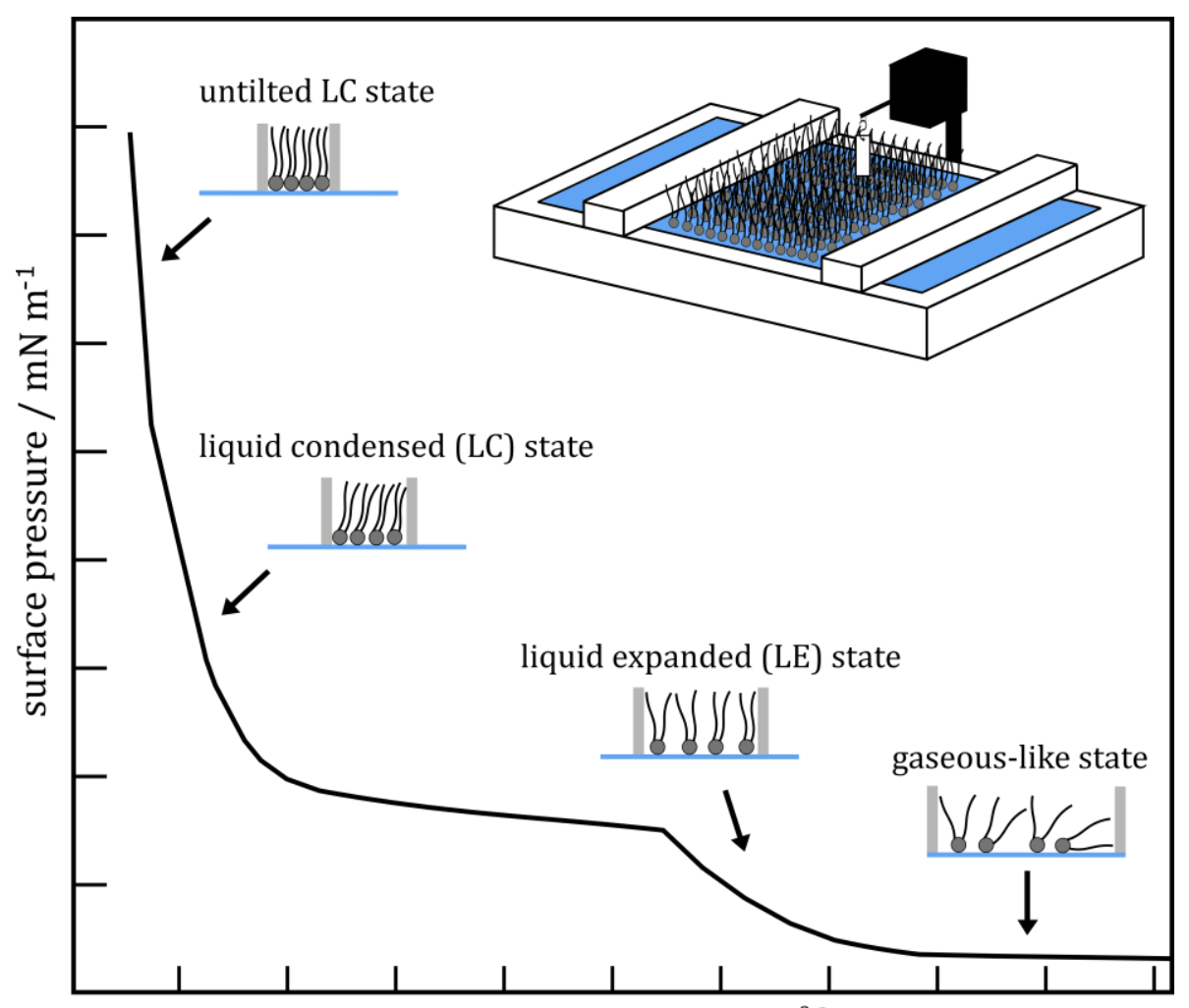

area per molecule / $\AA^{2}$

Figure 3.12: Schematic illustration of a Langmuir-Blodgett trough and a typical surface pressure-area per molecule isotherm. Due to compression the area per molecule gets smaller and the lipids change their orientation. The transition from a gas-like phase to a liquid-expanded state occurs. Further compression leads to a solid-like or untilted LC state via a liquid condensed (LC). The Figure was modified according to M Larsen. ${ }^{91}$

With a large trough area the lipid molecules were comparable to a $2 \mathrm{D}$ gas. ${ }^{92,93}$ By reducing the area the lipids switch to a liquid-expanded (LE) state. At a certain surface pressure a co-existence region of the LE and the liquid condensed (LC) phase can occur, induced by a first-order transition, followed by the LC state, where the lipids are highly ordered. Further compression leads to the collapse of the film due to too high packing densities. Here the appearance of multilayers or the formation of micelles are possible.

\section{Determination of the surface pressure}

When amphiphilic molecules, like lipids, get in contact with a water-air interface a rearrangement of the lipids occurs. The hydrophilic head groups will get in contact 
with the water (subphase) and the lipophilic tail protrudes into the air. This perpendicular alignment is associated with the decrease of the free energy of the system. ${ }^{94}$ An enrichment of the molecules causes the favored expansion of the interface with an interface or surface pressure $\pi$. The surface tension of the water $\gamma_{\mathrm{H}_{2} \mathrm{O}}(72.5 \mathrm{mN} / \mathrm{m})^{95}$ counteracts, resulting in a reduced surface tension. The surface pressure is hence the difference of the surface tension without $\gamma_{\mathrm{H}_{2} \mathrm{O}}$ and with the lipid film $\gamma_{\text {Film }}$ (equation $(3.21)) \cdot{ }^{96}$

$$
\gamma_{\text {Film }}=\gamma_{\mathrm{H}_{2} \mathrm{O}}-\pi \leftrightarrow \pi=\gamma_{\mathrm{H}_{2} \mathrm{O}}-\gamma_{\text {Film }}
$$

The resulting surface tension $\gamma_{\text {Film }}$ was determined with the Wilhelmy plate method. Figure 3.13 shows the principle of this technique.

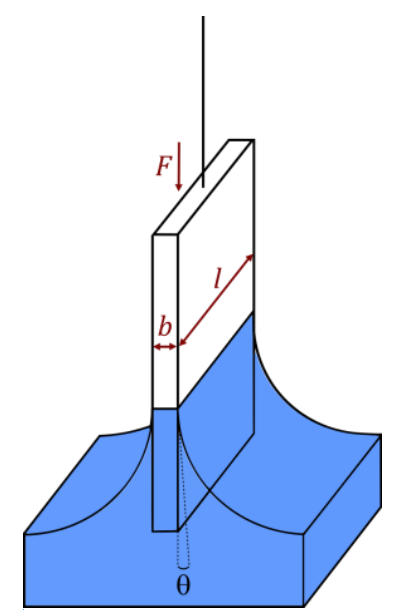

Figure 3.13: Wetting principle of a Wilhelmy plate. A force $F$ acts on the Wilhelmy plate, which is proportional to the wetted perimeter $L=(2 \mathrm{l}+2 \mathrm{~b}) . l$ and $b$ describe the plate width and thickness. The plate is wetted at an angle $\Theta$.

By wetting of a Wilhelmy plate a force $F$ acts on this plate according to equation (3.22).

$$
F=\gamma \cdot L \cdot \cos \Theta \leftrightarrow \gamma=\frac{F}{L \cdot \cos \Theta}
$$

This force correlates with the surface tension, the wetted perimeter $L=(2 l+2 b)$ (cf. Figure 3.13) and the contact angle $(\Theta)$. Wilhelmy plates are often made of filter papers, ensuring complete wetting $\left(\Theta=0^{\circ}\right)$, which simplifies the equation (3.22) to: 


$$
\gamma=\frac{F}{L} \rightarrow \gamma_{\text {Film }}=\frac{F}{L}
$$

The calculation of the surface tension $\gamma$, or more exactly the surface tension with the film $\gamma_{\text {Film }}$, then enables the determination of the surface pressure $\pi$ (cf. equation (3.21)), which is displayed on the control system.

\section{Experimental procedure}

Before the experiment was started, the trough and the barriers were cleaned with Mucasol for $15 \mathrm{~min}$. In this time the thermostat $\left(20^{\circ} \mathrm{C}\right)$ and the software were turned on and the filter paper was soaked in ultrapure water. After rinsing with ultrapure water (trough 4 times, barriers 10 times), the trough was filled with $120 \mathrm{~mL}$ subphase. Lipid concentrations were determined in ultrapure water and the investigation of the monolayer stability was performed in PBS. For measurements with a protein trough, first $50 \mathrm{~mL}$ PBS were added. The protein trough was then fixed with screws and further $70 \mathrm{~mL}$ PBS were filled into the middle of the trough. The barriers were fixed and the filter paper was dipped into the subphase to get a slight contact. During this contact the Offset was adjusted to $0 \mathrm{mN} / \mathrm{m}$. Without contact to the water, the Calibration-screw was set to $72.75 \mathrm{mN} / \mathrm{m}\left(\gamma_{\mathrm{H}_{2} \mathrm{O}}\right) .{ }^{95}$ This procedure was repeated three times for precise calibration.

The lipid mixture, dissolved in chloroform, was carefully added to the subphase with a Hamilton syringe, which was rinsed three times with chloroform before use. The system was then equilibrated for $15 \mathrm{~min}$ to remove the chloroform by vaporization. After adjusting the barrier velocity $\left(4.7 \mathrm{~mm}^{2} / \mathrm{s}\right)$ and starting the measurement in the software, the barrier motors (setup: "auto") were turned on.

For determination of the lipid concentrations via an isotherm the trough area was compressed up to a surface pressure of $\pi=25 \mathrm{mN} / \mathrm{m}$. For the isotherms of the lipid mixtures the surface pressure was set to $\pi=35 \mathrm{mN} / \mathrm{m}$. To investigate the monolayer stability of the used lipid mixtures a maximum surface pressure of $\pi=30 \mathrm{mN} / \mathrm{m}$ was adjusted. When this surface pressure was reached, the barrier motors were turned 
off to get a constant trough area. The surface pressure is then plotted against the time to see if the monolayer remained stable over one hour.

Protein insertion experiments were done with a special protein trough (Figure 3.14). This protein trough enables the insertion of ENTH into the subphase to investigate the surface activity in a smaller volume $(V=2 \mathrm{~mL})$.

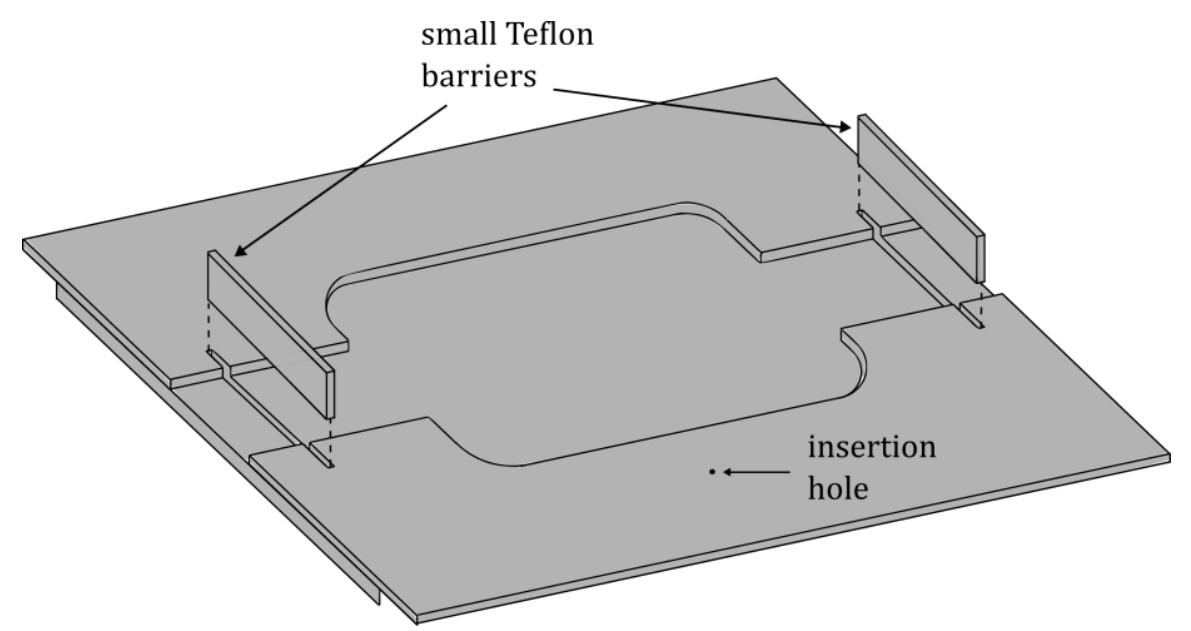

Figure 3.14: Schema of the used protein trough with a capacity of $2 \mathrm{~mL}$, confined by small Teflon barriers. Through a small hole protein can be inserted into the subphase.

For this experiment different initial surface pressures were set. When the desired pressure was achieved, the barrier motors were turned off and small Teflon barriers were fixed into the pre-built notches. As the monolayer is disturbed during the inset of the small barriers, the system was equilibrated for 5-10 min until no more change of the surface pressure was observed. Then $1 \mu \mathrm{M}$ of the ENTH-domain was added to the subphase and subsequently distributed with a Hamilton syringe. The measurements were recorded until a maximum of the surface pressure change was reached. In this work following lipid mixtures were used to analyze the ENTH-PIP 2 interaction with the film balance technique.

- $\quad$ POPC/POPE 80:20

- POPC/POPE/PIP2 79:20:1

- POPC/POPE/PIP2 75:20:5

- $\quad$ POPC/POPE/PIP2 70:20:10

- POPC/POPE/POPS 60:20:20

- $\quad$ POPC/POPE/POPS/PIP2 55:20:20:5 


\section{ReSUltS}

In this work, the impact of negatively charged lipids phosphatidylinositol-4,5bisphosphate $\left(\mathrm{PIP}_{2}\right)$ and phosphatidylserine (PS) on the interaction of the epsin $N$-terminal homology (ENTH) domain and membranes was analyzed. First, the binding behavior on solid supported bi- and monolayers was investigated by means of reflectometric interference spectroscopy (RIfS). Furthermore, atomic force microscopy (AFM) experiments were performed to obtain the surface topology of membranes after ENTH binding. Finally, the penetration capability of ENTH and its influence on membrane tension were determined using the Langmuir-Blodgett technique and spinning disc confocal laser microscopy (SDCLM).

\subsection{Influence of the PIP 2 concentration on the ENTH binding to solid supported lipid membranes}

In the clathrin-mediated endocytosis the receptor lipid $\mathrm{PIP}_{2}$ is necessary for the recruitment of ENTH to the membrane and consequently involved in one key step of this process (cf. chapter 1.1). 8,21,25 It is predicted that ENTH binds PIP2 in a 1:1 stoichiometry, ${ }^{7}$ which would assume a direct correlation of the $\mathrm{PIP}_{2}$ content and the amount of bound protein. The impact of $\mathrm{PIP}_{2}$ and especially its concentration on the ENTH-membrane interactions is still unexplored. To analyze how the concentration of the receptor lipid influences the protein occupancy on $\mathrm{PIP}_{2}$ doped membranes, RIfS experiments were performed. Therefore, the amount of bound protein on solid supported bilayers and monolayers at different PIP 2 concentrations was determined. 


\subsubsection{Formation of solid-supported lipid bilayers on silicon dioxide}

Since the formation of a homogenous and planar membrane is required to investigate the ENTH binding by means of RIfS, the spreading of small unilamellar vesicles (SUVs) on hydrophilic silicon dioxide $\left(\mathrm{SiO}_{2}\right)$ was considered first. When vesicles of POPC/PIP 2 with different receptor lipid concentrations (0-10 mol\%) were added to a hydrophilic $\mathrm{SiO}_{2}$ wafer, they spread on the surface, resulting in a lipid bilayer. During this spreading process the increase of the optical thickness $(\triangle O T)$ was observed, indicating the successful bilayer formation. In Figure 4.1 the formation of a POPC (100 mol\%, A) and a POPC/PIP2 (90:10, B) bilayer are shown.

A

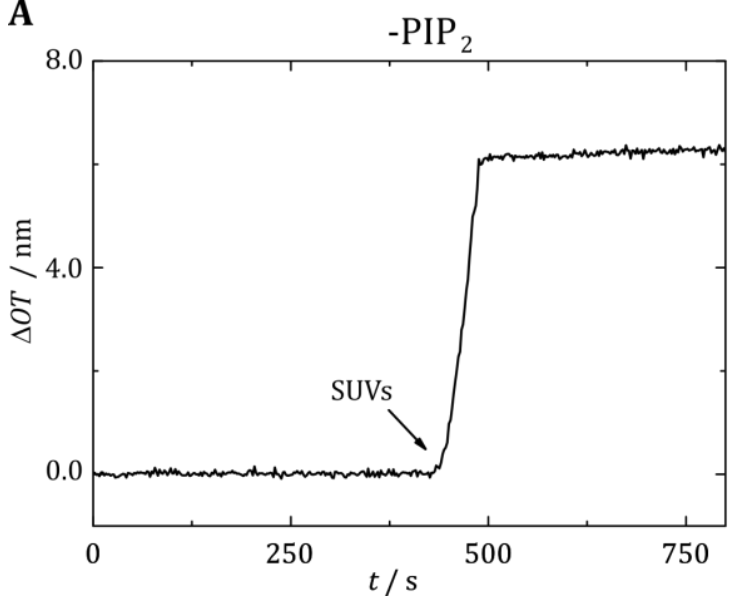

B

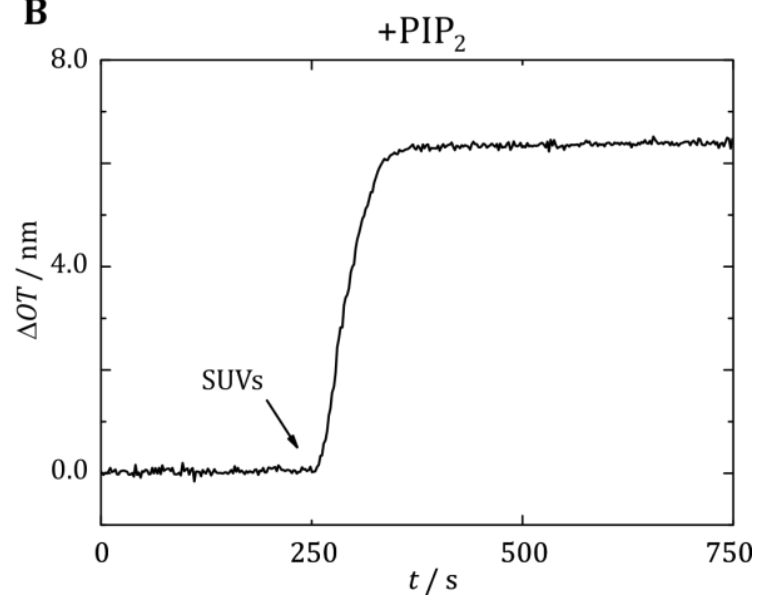

Figure 4.1: Exemplary plots of the change in optical thickness vs. the time during vesicle spreading of (A) pure POPC and (B) POPC/PIP2 (90:10) SUVs onto hydrophilic silicon dioxide wafers. During spreading of SUVs the optical thickness increases until a plateau is reached, indicating the successful formation of a bilayer.

The baseline was generated by rinsing the system with citrate buffer at a $\mathrm{pH}$ of 4.8 , which reduced the negative charge of the $\mathrm{PIP}_{2}$ and the $\mathrm{SiO}_{2}$ surface. In previous studies, it was shown that these conditions (ions, $\mathrm{pH}$ ) improve the spreading of $\mathrm{PIP}_{2}$ containing SUVs and hence the bilayer formation. ${ }^{97}$

After rinsing with PBS to remove non-spread vesicles, the surface was passivated with BSA to avoid defects in the bilayer. The system was then rinsed with PBS again, yielding the $\triangle O T$ after vesicle spreading (Figure 4.2). 


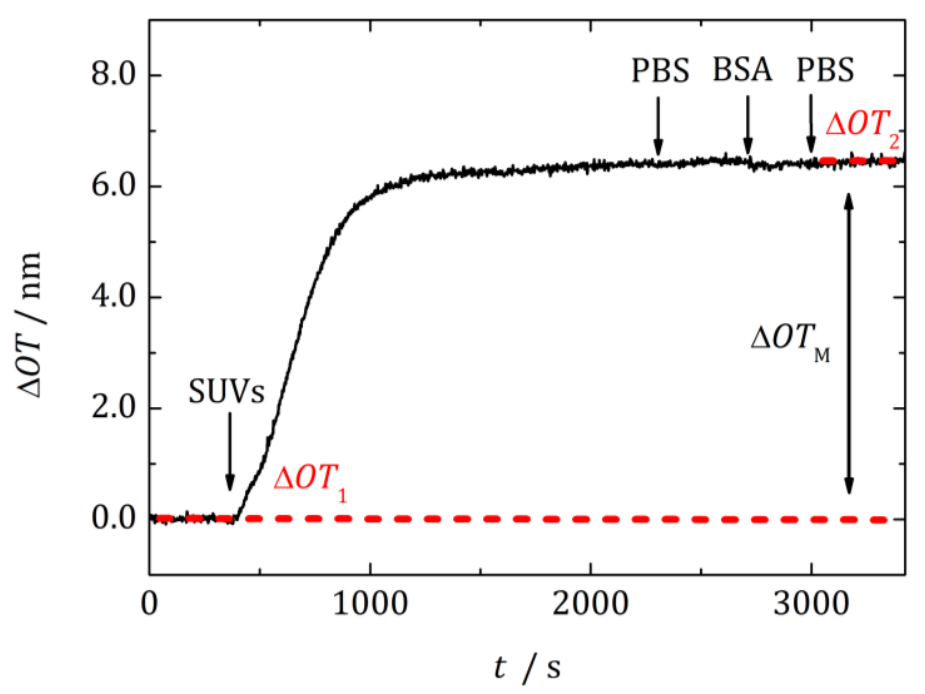

Figure 4.2: Time-trace of the spreading process of POPC/PIP2-SUVs, followed by rinsing with PBS and BSA. PBS was added to remove adhered, non-spread vesicles and BSA for passivation. The optical thickness of the membrane $\left(\triangle O T_{\mathrm{M}}\right)$ is determined by the difference of $\Delta O T_{2}-\Delta O T_{1}$. Here an example of the bilayer formation on hydrophilic $\mathrm{SiO}_{2}$ is shown. The $\Delta O T_{\mathrm{M}}$ of a monolayer is calculated analogously.

To obtain the membrane thickness $\left(\triangle O T_{\mathrm{M}}\right)$ the difference of the $\triangle O T$ before (baseline, $\left.\triangle O T_{1}\right)$ and after $\left(\triangle O T_{2}\right)$ SUV spreading were calculated with equation (4.1).

$$
\Delta O T_{\mathrm{M}}=\Delta O T_{2}-\Delta O T_{1}
$$

From the optical thickness of the membrane $\left(\triangle O T_{\mathrm{M}}\right)$ the physical thickness $d_{\mathrm{M}}$ could be determined with equation (3.7).

The lipid bilayer formation was investigated for different receptor lipid concentrations in the vesicles $\left.\left(\chi \mathrm{PIP}_{2}=0-10 \mathrm{~mol} \%\right)\right)$. In Table 4.1 the determined values for $\Delta O T_{\mathrm{M}}$ and $d_{\mathrm{M}}$ were collected. 
Table 4.1: Averaged optical and physical thicknesses of the POPC/PIP 2 lipid bilayers with the corresponding standard deviations. The receptor lipid concentration was changed from 0-10 mol\%. $n$ number of performed experiments.

\begin{tabular}{|c|c|c|c|}
\hline Lipid composition & $\Delta O T_{\mathrm{M}} / \mathrm{nm}$ & $d_{\mathrm{M}} / \mathrm{nm}$ & $n$ \\
\hline POPC & $6.4 \pm 0.2$ & $4.4 \pm 0.2$ & 5 \\
\hline POPC/PIP2 (99:1) & $6.4 \pm 0.3$ & $4.3 \pm 0.2$ & 4 \\
\hline POPC/PIP2 (98:2) & $6.4 \pm 0.4$ & $4.3 \pm 0.3$ & 8 \\
\hline POPC/PIP2 (95:5) & $6.4 \pm 0.5$ & $4.3 \pm 0.4$ & 9 \\
\hline POPC/PIP2 (93:7) & $6.3 \pm 0.2$ & $4.3 \pm 0.2$ & 4 \\
\hline POPC/PIP2 (92:8) & $6.3 \pm 0.4$ & $4.3 \pm 0.3$ & 5 \\
\hline POPC/PIP2 (90:10) & $6.3 \pm 0.5$ & $4.3 \pm 0.3$ & 10 \\
\hline
\end{tabular}

The $\triangle O T$ of the membranes ranged from 6.3 to $6.4 \mathrm{~nm}$ and the $d \mathrm{~m}$ from 4.3 to $4.5 \mathrm{~nm}$, not showing a significant change with increased $\mathrm{PIP}_{2}$ concentration as it was presumed due to the larger head group of $\mathrm{PIP}_{2}{ }^{44,98}$ These results show that for all lipid compositions the bilayers were formed successfully.

To verify that the formed lipid bilayers are homogenous and mobile, additionally fluorophore labeled bilayers were investigated by means of confocal laser scanning microscopy (CLSM). Solid supported lipid bilayers composed of POPC, PIP2 (1-10 mol\%) and the lipid coupled fluorophore Texas $\operatorname{Red}^{\circledR}$ 1,2-dihexadecanoyl-snglycero-3-phosphoetanolamine (TxR) were prepared as described in chapter 3.2.2. Then FRAP-experiments were performed to obtain the diffusion coefficients $(D)$ of TxR in the solid supported bilayers (cf. chapter 3.3.3.1). In Figure 4.3 an exemplary FRAP experiment on POPC/PIP 2 /TxR (89.5:10:5) is shown. 
A

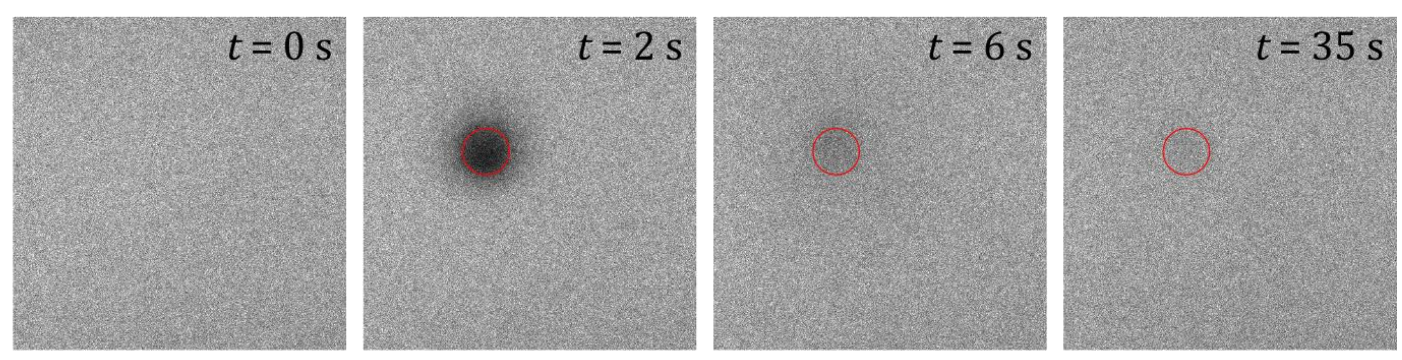

B

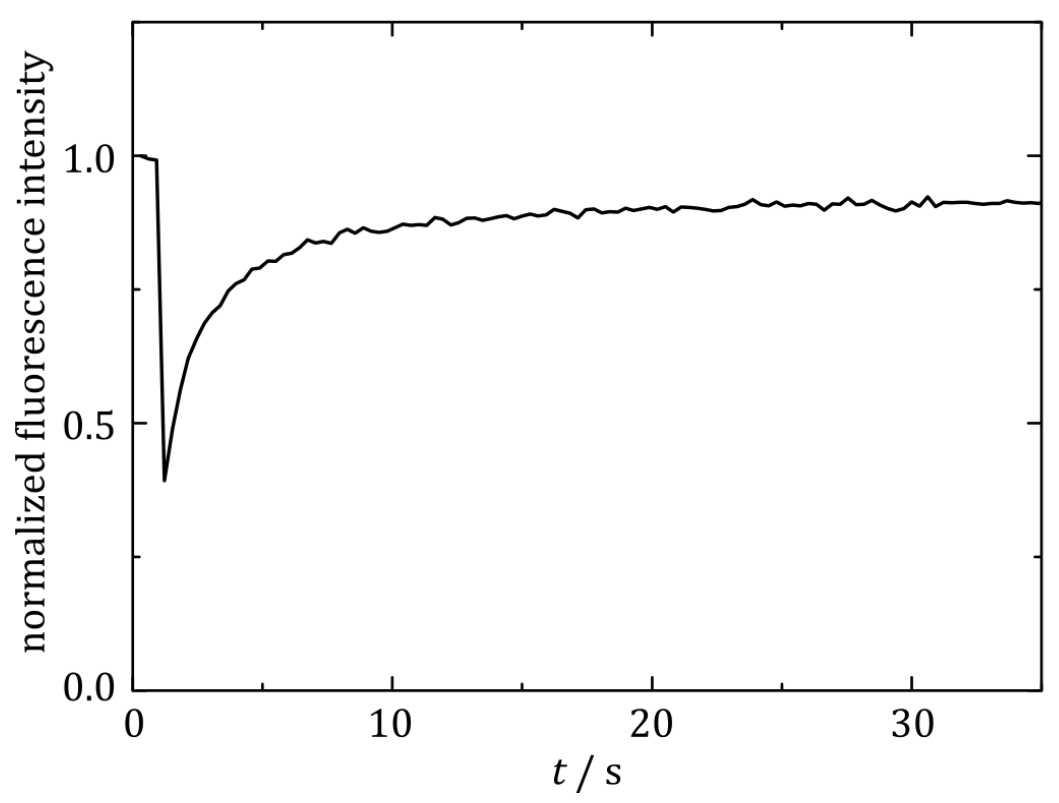

Figure 4.3: (A) Fluorescence images of a FRAP experiment and (B) the corresponding time-trace of the normalized fluorescence intensity on a POPC/PIP $/$ TxR (89.5:10:0.5) bilayer. After bleaching of a region of interest (ROI, red circle, $t=2 \mathrm{~s}$ ) the fluorescence intensity increases, reaching a plateau after $20 \mathrm{~s}$. Due to the immobile fraction of TxR in the bilayer, the fluorescence intensity does not reach the fluorescence intensity value at $t=0$.

In all samples (1-10 mol\% $\left.\mathrm{PIP}_{2}\right)$ the fluorescence of TxR was distributed equally, indicating the formation of a homogenous bilayer without obvious defects. A region of interest (ROI) of the bilayer was bleached and the fluorescence recovery was recorded. All membranes were mobile as the fluorescence intensity increased over time. The diffusion coefficients were determined using a MatLab Scipt from Jönssen et $a .^{78} \mathrm{In}$ Table 4.2 the determined diffusion coefficients and the mobile fractions are listed. 
Table 4.2: Mean values of the diffusion coefficients $D$ and the mobile fractions $F_{\mathrm{m}}$ of TxR in lipid bilayers with corresponding standard deviations yielded by FRAP experiments. $n$ is the amount of performed FRAP experiments.

\begin{tabular}{|c|c|c|c|}
\hline $\begin{array}{c}\text { Lipid composition } \\
\text { POPC/PIP } / T x R\end{array}$ & $\begin{array}{c}\text { Diffusion coefficient } \\
D / \mu \mathrm{m}^{2} / \mathrm{s}\end{array}$ & $\begin{array}{c}\text { Mobile fraction } \\
F_{\mathrm{m}} / \%\end{array}$ & $n$ \\
\hline $98.5: 1: 0.5$ & $2.2 \pm 0.3$ & $99 \pm 1$ & 8 \\
\hline $97.5: 2: 0.5$ & $2.3 \pm 0.2$ & $98 \pm 1$ & 7 \\
\hline $94.5: 5: 0.5$ & $2.3 \pm 0.3$ & $93 \pm 4$ & 7 \\
\hline $92.5: 7: 0.5$ & $1.8 \pm 0.3$ & $89 \pm 4$ & 8 \\
\hline $91.5: 8: 0.5$ & $2.5 \pm 0.4$ & $90 \pm 2$ & 7 \\
\hline $89.5: 10: 0.5$ & $2.1 \pm 0.6$ & $90 \pm 3$ & 7 \\
\hline
\end{tabular}

The diffusion coefficient of the bilayers ranged from $1.8-2.5 \mu \mathrm{m}^{2} / \mathrm{s}$. Within the standard deviation the values were similar and did not show a trend with increasing receptor lipid concentration. Considering the mobile fractions of the lipid bilayers the $\mathrm{PIP}_{2}$ concentration seemed to have a minor influence. The mobile fraction generally slightly decreased from (99 \pm 1 ) \% for 1 mol\% $\mathrm{PIP}_{2}$ to $(90 \pm 3) \%$ for $10 \mathrm{~mol} \% \mathrm{PIP}_{2}$. The values were significantly different (Mann-Whitney $U$ test, $p<0.01$ ). Together, the results indicate successful formation of homogenous and mobile lipid bilayers.

\subsubsection{Formation of solid-supported lipid monolayers on silicon dioxide}

Supported lipid bilayers are a versatile tool to investigate membrane-protein interaction as they exhibit high mechanical stability. However, the substrate surface can influence the lipid distribution within the both leaflets ${ }^{99,100}$. This can have an effect on the accessibility of specific receptor lipids on the upper leaflet of the SLBs, necessary for protein binding. To investigate if the $\mathrm{PIP}_{2}$ is asymmetrically distributed on supported bilayers and the ENTH binding is influenced, RIfS experiments with monolayers were done. In monolayers the receptor lipid should be distributed homogenously and completely accessible for ENTH binding due to the missing second leaflet. 
For precise binding studies of ENTH the formation of a stable monolayer is required. SUVs composed of POPC and PIP2 (0-10 mol\%) were spread on hydrophobic functionalized silicon dioxide substrates (cf. chapter 3.2.2), leading to an increase of the $\triangle O T$. Figure 4.4 shows exemplary RIfS time-traces of the spreading process of POPC (100 mol\%) and POPC/PIP 2 (90:10) vesicles on these substrates.
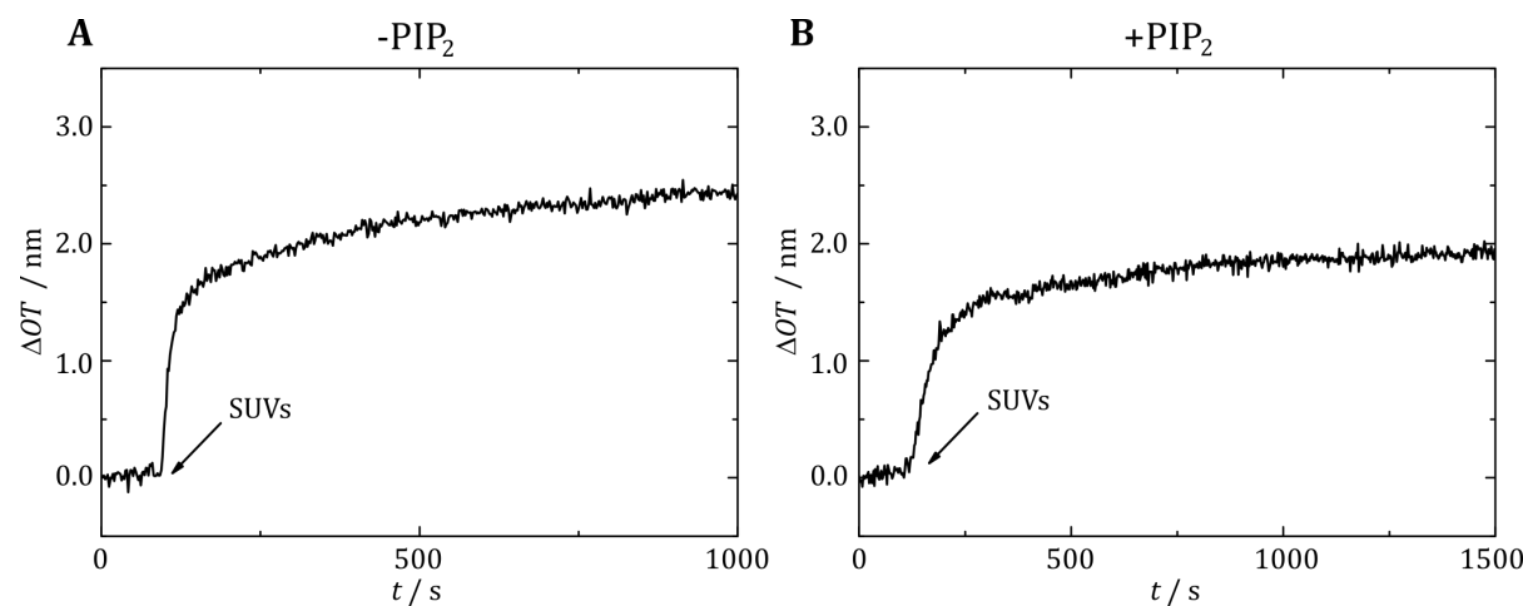

Figure 4.4: Exemplary RIfS time-traces of the vesicle spreading on hydrophobic functionalized silicon dioxide. Pure POPC (A) and POPC/PIP2 (90:10, B) SUVs were added after a baseline with citrate buffer was observed, resulting in the formation of a lipid monolayer indicated by the $\triangle O T$ increase of about 2-3 $\mathrm{nm}$.

After the formation of a baseline with the citrate buffer (cf. chapter 4.1.1) in either case the addition of SUVs led to the $\triangle O T$ increase up to 2.1-2.6 $\mathrm{nm}$ indicating that the bilayer was formed successfully. First, $\triangle O T$ increased fast, then slighter reaching a plateau. The lipid monolayer formation was investigated for different $\mathrm{PIP}_{2}$ concentrations $c\left(\mathrm{PIP}_{2}=0-10 \mathrm{~mol} \%\right)$. In Table 4.3 the determined values for $\triangle O T$ and $d \mathrm{~m}$ were listed. 
Table 4.3: The optical and physical thicknesses of the POPC/PIP2 lipid monolayers with receptor lipid concentration ranging from $0-10 \mathrm{~mol} \%$. The errors were given as standard deviations. $n$ is the number of measurements.

\begin{tabular}{|c|c|c|c|}
\hline Lipid composition & $\Delta O T / \mathrm{nm}$ & $d_{\mathrm{M}} / \mathrm{nm}$ & $n$ \\
\hline POPC & 2.7 & $1.6 \pm 0.2$ & 3 \\
\hline POPC/PIP2 (99:1) & $2.6 \pm 0.3$ & $1.8 \pm 0.2$ & 4 \\
\hline POPC/PIP2 (98:2) & $2.6 \pm 0.6$ & $1.7 \pm 0.4$ & 5 \\
\hline POPC/PIP2 (95:5) & $2.1 \pm 0.5$ & $1.4 \pm 0.3$ & 3 \\
\hline POPC/PIP2 (93:7) & $2.4 \pm 0.7$ & $1.6 \pm 0.4$ & 3 \\
\hline POPC/PIP2 (92:8) & $2.4 \pm 0.3$ & $1.7 \pm 0.2$ & 6 \\
\hline POPC/PIP2 (90:10) & $2.3 \pm 0.2$ & $1.6 \pm 0.2$ & 3 \\
\hline
\end{tabular}

In all cases the monolayers were formed successfully with physical thicknesses of 1.4 to $1.8 \mathrm{~nm}$. The experiments showed no significant influence of the $\mathrm{PIP}_{2}$ concentration on the membrane thickness within the standard deviations.

Since interactions of membrane layers with the substrate surface can occur, which influence the membrane mobility, ${ }^{99}$ diffusion coefficients of TxR in the lipid monolayers were determined by FRAP. Fluorescence images of POPC/PIP $2 / T x R$ monolayers with different PIP2 concentrations (1 - $10 \mathrm{~mol} \%$ ) revealed an even distribution of the fluorophore, indicating that homogenous monolayers were formed. Rarely occurring small bright spots were assigned to adhered vesicles. In Figure 4.5 exemplary fluorescence images of a FRAP experiment on POPC/PIP2/TxR (89.5/10/0.5) and the corresponding time-trace of the normalized fluorescence intensity are illustrated. 
A

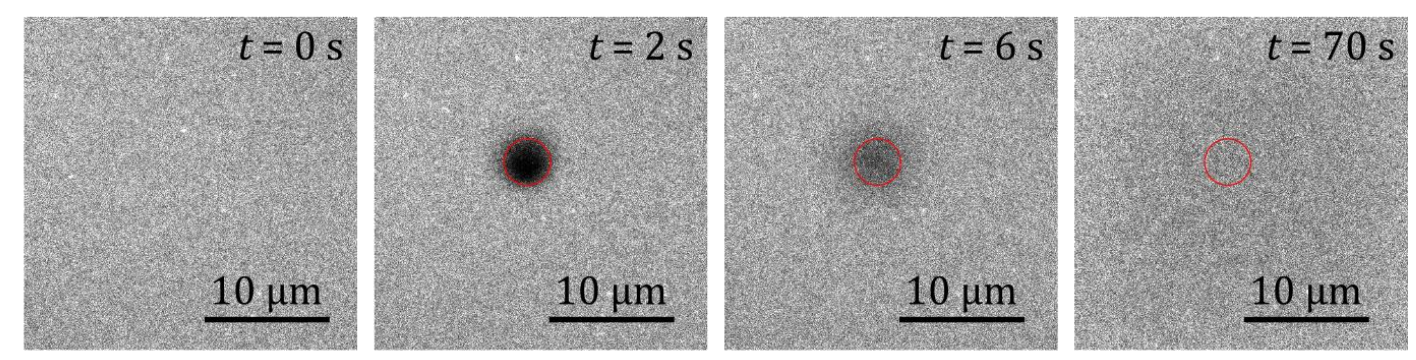

B

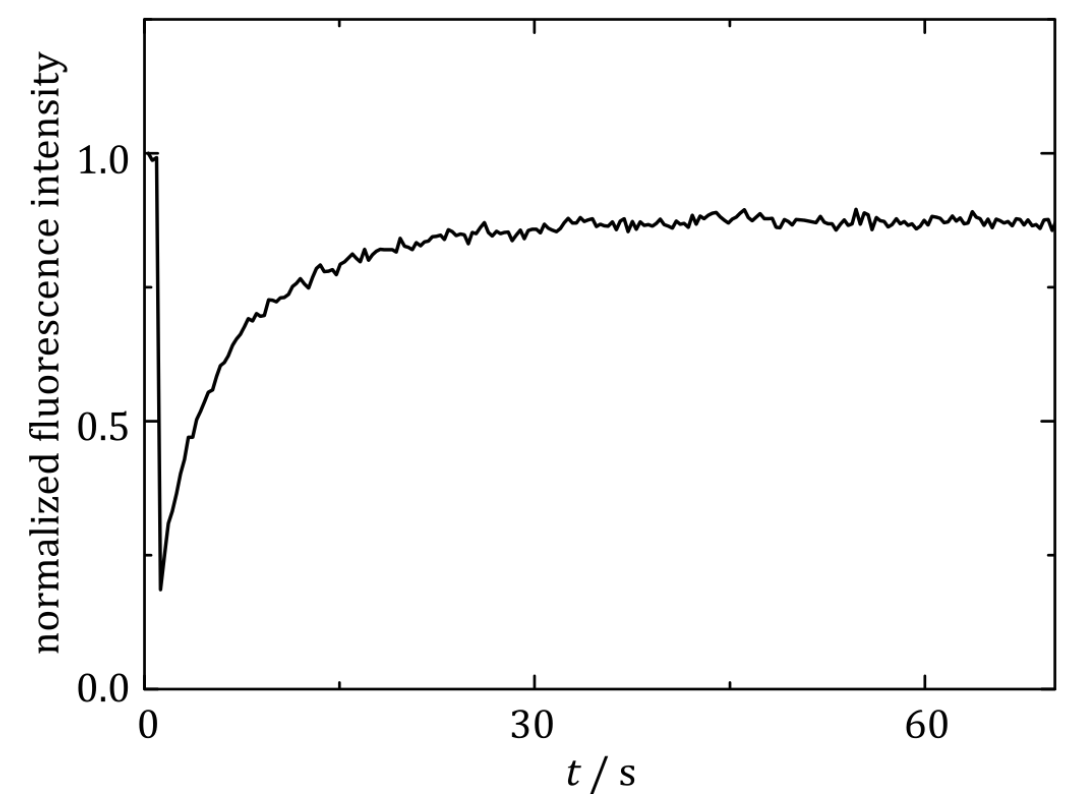

Figure 4.5: (A) Fluorescence images of a FRAP experiment and (B) the corresponding time-trace of the normalized fluorescence intensity on a POPC/PIP/TxR (89.5:10:0.5) monolayer. After bleaching of a ROI (red circle, $t=2 \mathrm{~s}$ ) the fluorescence intensity increases, reaching a plateau after $40 \mathrm{~s}$. Due to the immobile fraction of TxR in the bilayer, the fluorescence intensity does not reach the fluorescence intensity value at $t=0$.

Bleaching of a ROI led to the decrease of the fluorescence intensity (Figure 4.5 B). By evaluating the fluorescence recovery with the MatLab Scrip of Jönsson et al. ${ }^{78}$ the diffusion coefficients and the mobile fractions $\left(F_{\mathrm{m}}\right)$ of the fluorophore were determined (Table 4.4). 
Table 4.4: Mean values of the diffusion coefficients $D$ and the mobile fractions $F_{\mathrm{m}}$ of the fluorophores in lipid monolayers with corresponding standard deviations yielded by FRAP experiments. $n$ is the amount of performed FRAP experiments.

\begin{tabular}{|c|c|c|c|}
\hline $\begin{array}{c}\text { Lipid composition } \\
\text { POPC/PIP } / T x R\end{array}$ & $\begin{array}{c}\text { Diffusion coefficient } \\
D / \mu \mathrm{m} 2 / \mathrm{s}\end{array}$ & $\begin{array}{c}\text { Mobile fraction } \\
F_{\mathrm{m}} / \%\end{array}$ & $n$ \\
\hline $98.5: 1: 0.5$ & $0.36 \pm 0.02$ & $98 \pm 1$ & 5 \\
\hline $97.5: 2: 0.5$ & $0.37 \pm 0.03$ & $98 \pm 1$ & 6 \\
\hline $94.5: 5: 0.5$ & $0.36 \pm 0.03$ & $95 \pm 2$ & 5 \\
\hline $92.5: 7: 0.5$ & $0.41 \pm 0.02$ & $98.0 \pm 0.1$ & 6 \\
\hline $91.5: 8: 0.5$ & $0.42 \pm 0.02$ & $98 \pm 3$ & 6 \\
\hline $89.5: 10: 0.5$ & $0.41 \pm 0.02$ & $98 \pm 8$ & 6 \\
\hline
\end{tabular}

The diffusion coefficient of $\mathrm{TxR}$ in all monolayers was calculated to be $D=0.4+0.1 \mu \mathrm{m}^{2} / \mathrm{s}(n=34)$. Moreover, mobile fractions of $F_{\mathrm{m}}=98 \pm 2 \%(n=34)$ were determined. For both values no influence of the receptor lipid content was observed. However these results indicate that the hydrophobic substrate surface affects the lateral diffusion of artificial membranes as the bilayer showed higher diffusion coefficient values (cf. section 4.1.1)

\subsubsection{Analysis of the ENTH binding on lipid mono- and bilayers}

It is known that the ENTH domain binds specifically to $\mathrm{PIP}_{2},{ }^{8,23}$ but the effect of the receptor lipid concentration on this binding is poorly investigated yet. Furthermore also the substrate seems to have a significant influence on the lipid distribution ${ }^{99}$ and mobility and consequently on the protein adsorption to membranes. Therefore, the binding of ENTH to POPC/PIP 2 bi- and monolayers with varying $\mathrm{PIP}_{2}$ content was analyzed via RIfS experiments. After the successful bi- and monolayer formation (cf. chapter 4.1.1 and 4.1.2), $1 \mu \mathrm{M}$ ENTH was added. In Figure 4.6 a schematic illustration of a whole RIfS experiment is shown. 


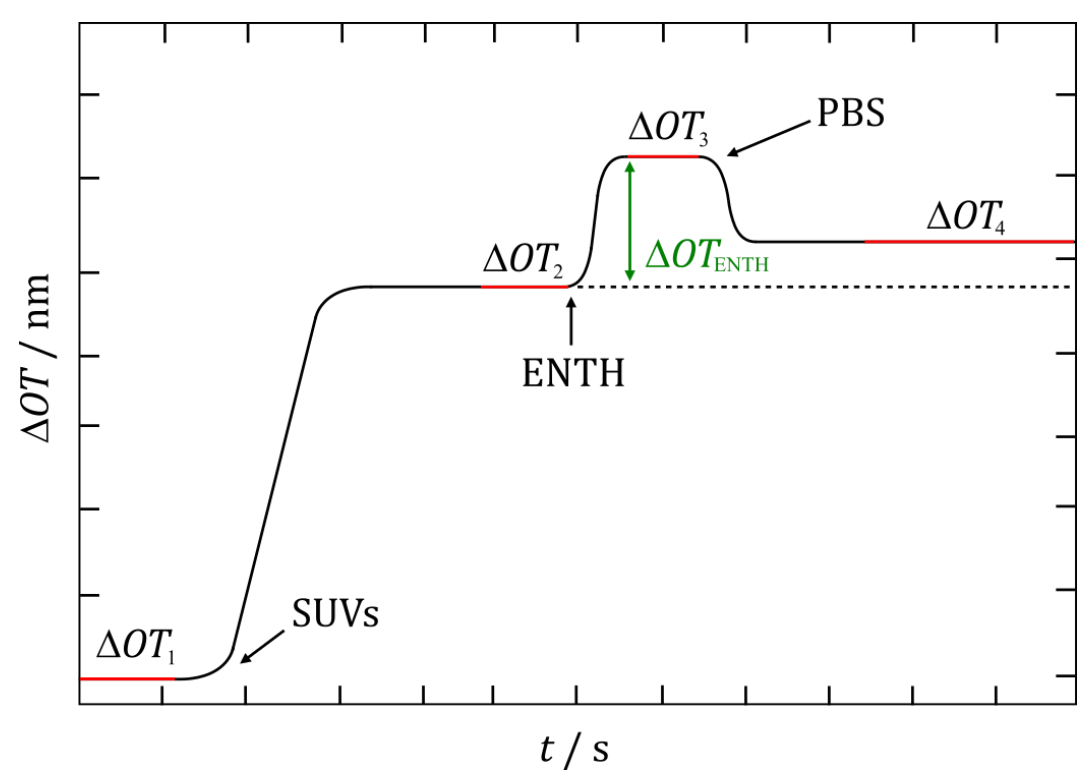

Figure 4.6: Schematic illustration of a RIfS experiment. After formation of a membrane ( $\left.\triangle O T_{2}\right)$ by SUV spreading, protein was added, resulting in a second increase of $\triangle O T$. By rinsing with PBS ENTH desorbed from the surface, reaching a new plateau $\left(\triangle O T_{4}\right)$. The optical thickness of the protein $\left(\triangle O T_{\text {ENTH }}\right)$ was determined with the difference of $\triangle O T_{3}$ and $\triangle O T_{2}$.

Addition of ENTH resulted in the increase of optical thickness. The difference of the $\triangle O T$ after protein adsorption $\left(\triangle O T_{3}\right)$ and $\triangle O T_{2}$ yielded the $\triangle O T$ of the protein ( $\triangle O T_{\mathrm{ENTH}}$, equation (4.3)), which can be translated into the physical thickness of the protein at complete surface coverage $\left(d_{\mathrm{ENTH}}\right.$, equation (3.7), with a refractive index $n_{\text {proteins }}=1.4772$ ).

$$
\Delta O T_{\mathrm{ENTH}}=\Delta O T_{3}-\Delta O T_{2}
$$

Dissociation of the ENTH domain from the membrane, bi- or monolayer, was reached by rinsing the system with PBS. The reversibility of the ENTH binding to $\mathrm{PIP}_{2}$ was calculated using equation (4.3).

$$
\text { Reversibility } / \%=\frac{\Delta O T_{3}-\Delta O T_{4}}{\Delta O T_{3}-\Delta O T_{2}}
$$

In order to show the specificity of the ENTH-PIP 2 binding, the experiments were first performed with pure POPC bi- and monolayers. In Figure 4.7 the time-resolved change in optical thickness during the ENTH addition is illustrated. To highlight the 
changes upon ENTH incubation the time and $\triangle O T$ after bi- and monolayer formation $\left(\Delta O T_{2}\right)$ in the figures were set to zero henceforth.

A

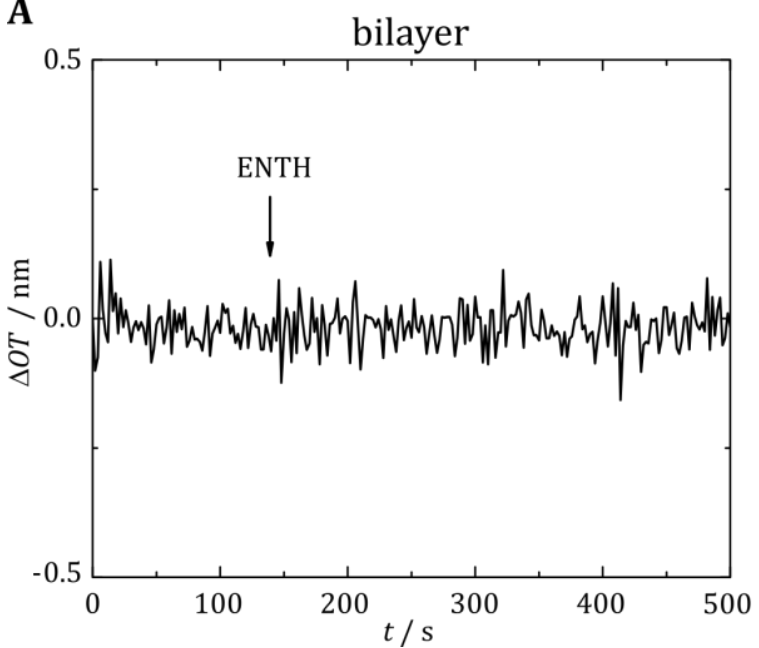

B

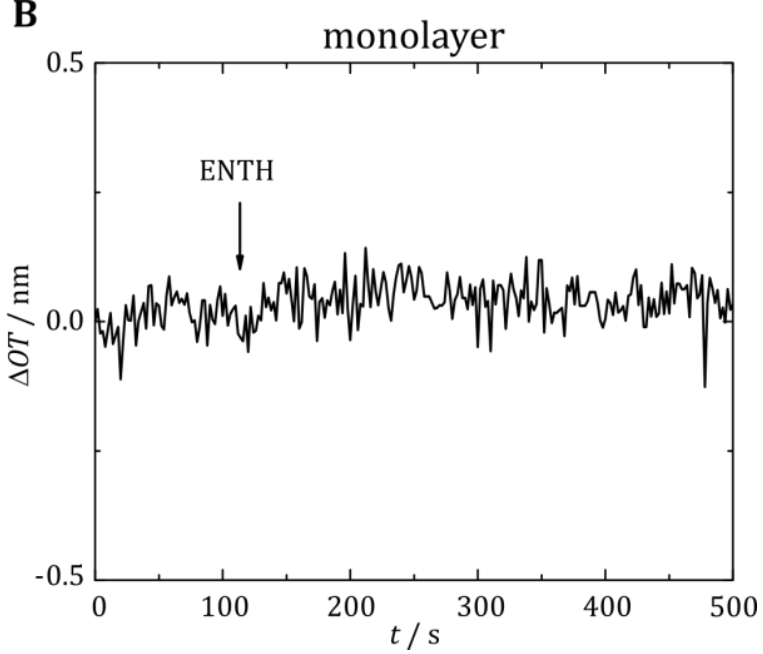

Figure 4.7: RIfS time-trace of the ENTH incubation $(1 \mu \mathrm{M})$ to pure POPC (A) bi- and (B) monolayers. After the protein addition no increase was observed, indicating that no ENTH was bound.

In both cases even after some time no increase of the optical thickness was observed (Figure 4.7), showing the specific binding of the ENTH domain to $\mathrm{PIP}_{2}$ as no protein was bound in the absence of the receptor lipid. Afterwards the ENTH binding to POPC/PIP2 mono- and bilayers dependent on the $\mathrm{PIP}_{2}$ concentration was investigated. The content of the receptor lipid therefore was varied from one to ten mol\%. The exemplary changes in $\triangle O T$ upon ENTH binding to POPC/PIP2 (92:8) mono- and bilayers are illustrated in Figure 4.8. 
A

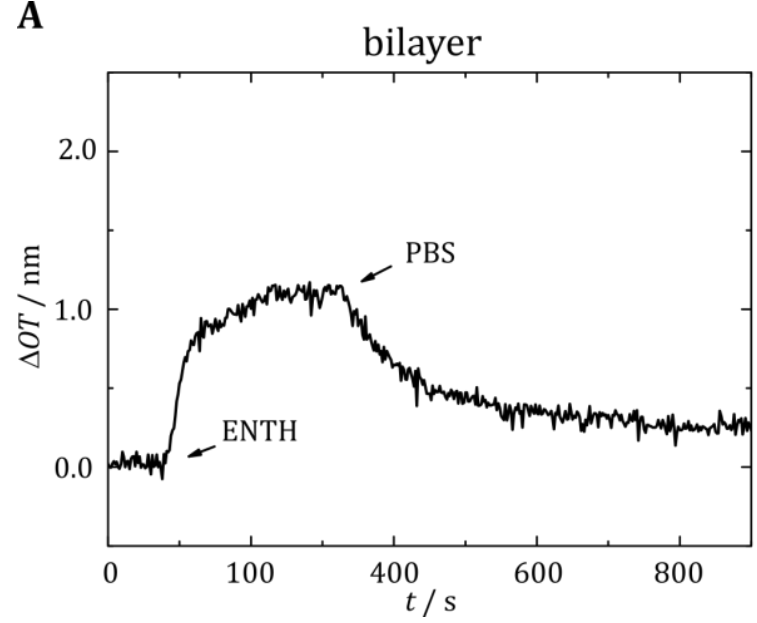

B

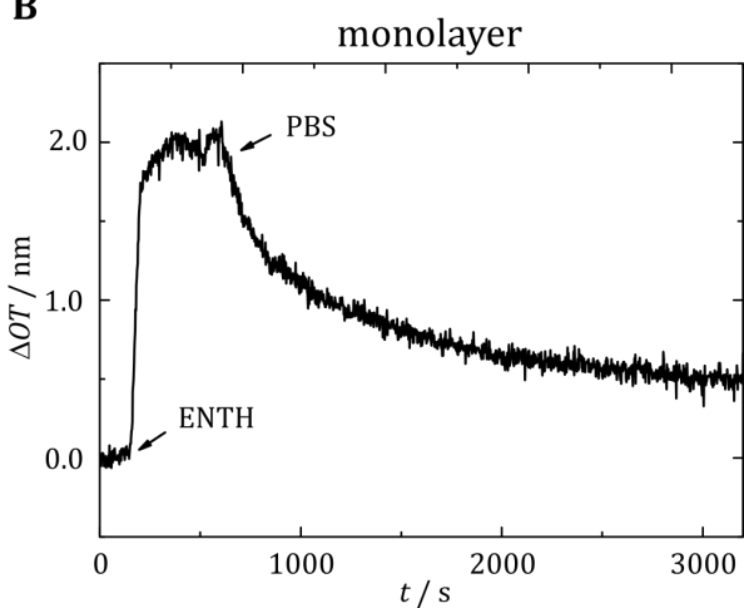

Figure 4.8: Representative time-resolved change in optical thickness observed by means of RIfS. showing the specific binding of $1 \mu \mathrm{M}$ ENTH to a (A) bi- and (B) monolayer composed of POPC/PIP2 (92:8).

In either case $\triangle O T$ first increased fast and then reached a plateau when ENTH was added. For the bilayer the $\triangle O T$ in the example increased up to $1.1 \mathrm{~nm}$. Compared to that protein adsorption to the monolayer resulted in a change in $\triangle O T$ up to $2.0 \mathrm{~nm}$, being about twice as high under the same conditions. This on the one hand shows, that $\mathrm{PIP}_{2}$ is necessary for ENTH binding to lipid membranes (Figure 4.8) and on the other hand proves the influence of the used membrane system (bilayer vs. monolayer) on binding. Afterwards rinsing with PBS led to the decrease of $\triangle O T$ in both cases, indicating reversible binding (Figure 4.8, Table 10.2).

Subsequently further binding studies were done for the other lipid compositions (0-10 mol\%). Based on these experiments $\triangle O T_{\text {ENTH }}$ was calculated using equation (4.3). In Figure 4.9, the time-resolved change in $\triangle O T$ upon protein addition and the obtained averaged $\triangle O T_{\text {ENTH }}$ values on bi- and monolayers are plotted for the different $\mathrm{PIP}_{2}$ concentrations. 
A

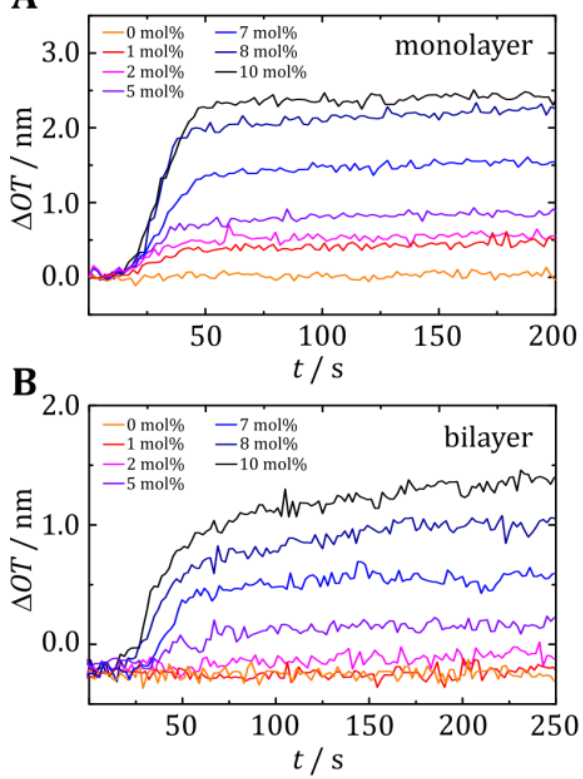

C

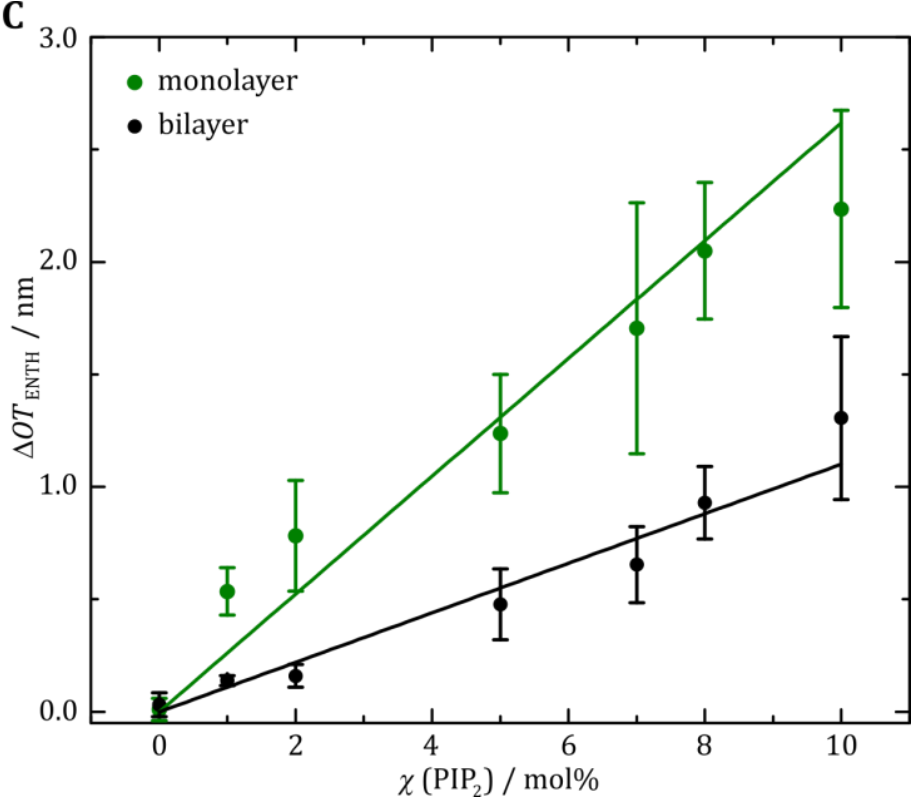

Figure 4.9: Time-resolved increase of $\triangle O T$ upon binding of $1 \mu \mathrm{M}$ ENTH to POPC (A) monolayers and (B) bilayers doped with PIP2 (0-10 mol\%). (C) Determined maximum changes in optical thickness after ENTH addition obtained from (A) and (B) plotted against the PIP2 content. The error bars in (C) are given as standard deviations. A linear fit weighted by the standard deviation was plotted trough the data. The mean values with standard deviation and the number of measurements for each lipid composition are listed in Table 10.1 (cf. Appendix).

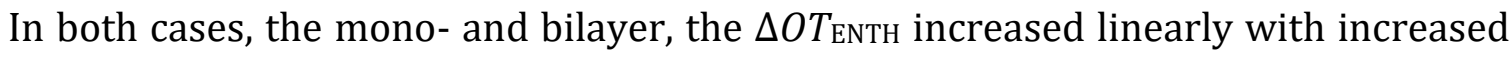
receptor lipid concentration, showing the significant influence of the PIP 2 content. Under same conditions the amount of bound protein to POPC/PIP 2 monolayers was about twice as high as for the bilayers. $\triangle O T_{\mathrm{ENTH}}$ for the monolayers with $10 \mathrm{~mol} \% \mathrm{PIP} 2$ reached a value of $2.2 \pm 0.4 \mathrm{~nm}$, indicating the beginning of a saturation as the values for $8 \mathrm{~mol} \%$ ( $2.0 \pm 0.3 \mathrm{~nm})$ and $10 \mathrm{~mol} \% \mathrm{PIP}_{2}$ are quite similar within the error. For the bilayers $\triangle O T_{\text {ENTH }}$ at $10 \mathrm{~mol} \%$ was determined to be $1.2 \pm 0.4 \mathrm{~nm}$. A saturation cannot be excluded within the errors.

Using equation (4.3) the reversibility of the ENTH binding to both membrane systems were calculated for the different $\mathrm{PIP}_{2}$ concentrations (Figure 4.10). 


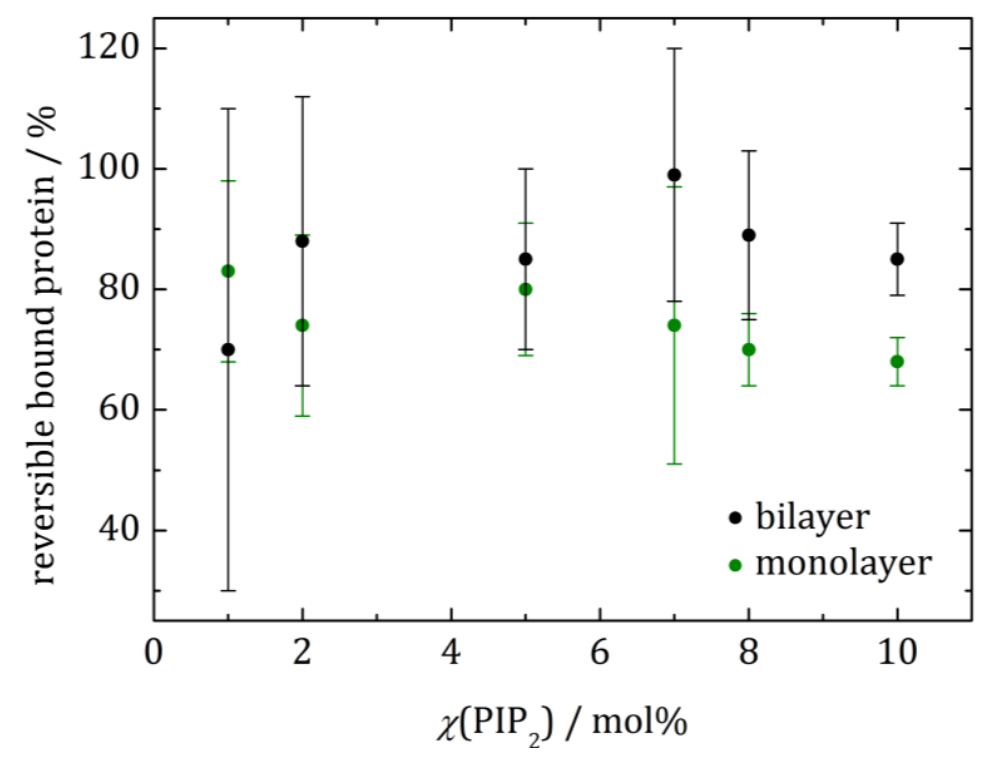

Figure 4.10: Reversibility of the ENTH binding to POPC/PIP 2 bi- and monolayers as a function of the $\mathrm{PIP}_{2}$ concentration, indicating that a high amount of the protein binds reversible to $\mathrm{PIP}_{2}$ doped membranes. The mean values with standard deviation and the number of measurements for each lipid composition are listed in Table 10.2 (cf. Appendix)

70-99\% of the ENTH bound reversibly to the bilayer. No trend dependent on the PIP 2 concentration was observed. Having the noise to signal ratio in mind, qualified statements of the $\triangle O T$ at low $\mathrm{PIP}_{2}$ concentrations are difficult to make as the $\triangle O T_{\text {ENTH was }}$ only $0.1 \pm 0.1 \mathrm{~nm}$ at a receptor lipid concentration of $1 \mathrm{~mol} \%$. This systematical error also explains the high deviations when working with low $\mathrm{PIP}_{2}$ concentrations.

Contrary to that the reversibility of the ENTH binding to monolayers showed a slight tendency. With increasing PIP2 content the reversibility slightly decreased from $83 \pm 15 \%(1 \mathrm{~mol} \%)$ to $68 \pm 4 \%(10 \mathrm{~mol} \%)$, but within the error margins the differences were negligible (Figure 4.10). Using a Mann-Whitney U test revealed that the values are not significantly different $(p=0.3)$.

In general the higher amount of bound protein to the monolayers showed that more $\mathrm{PIP}_{2}$ was accessible in the monolayers compared to the bilayers under the same conditions. 


\subsection{The influence of PS on the ENTH binding to PIP 2 containing solid supported lipid bilayers}

Not only the $\mathrm{PIP}_{2}$ content in the membrane has an influence on the amount of bound ENTH, also other lipids showed to cause an increased protein binding. Hom et al. performed liposome binding assays, which evidenced the higher affinity of ENTH to SUVs composed of $\mathrm{PC} / \mathrm{PE} / \mathrm{PS} / \mathrm{C}_{16}-\mathrm{PIP}_{2}$ compared to SUVs lacking PS. ${ }^{8}$ Moreover, B. Kroppen presented a co-sedimentation assay to show the appearance of protein homo-oligomers in liposomes in the presence of PS. ${ }^{26}$

In this chapter the impact of PS on the binding affinity of ENTH to $\mathrm{PIP}_{2}$ doped lipid bilayers was analyzed via RIfS. Furthermore AFM experiments were performed to investigate the surface topology of bilayers after ENTH incubation.

\subsubsection{Influence of PS on the bilayer formation}

For exact binding studies of ENTH to PS-containing lipid bilayers by means of RIfS the formation of a homogenous and mobile membrane is requisite. Since the lipid composition was changed by adding the negatively charged lipid PS, the quality of the formed bilayer and the thickness can be influenced. Therefore, SUVs composed of POPC/POPS (80:20) and POPC/POPS/PIP2 (75:20:5) were spread on hydrophilic silicon dioxide (see Figure 4.11), resulting in the $\triangle O T$ increase. In all cases SUV spreading was performed in citrate buffer. 

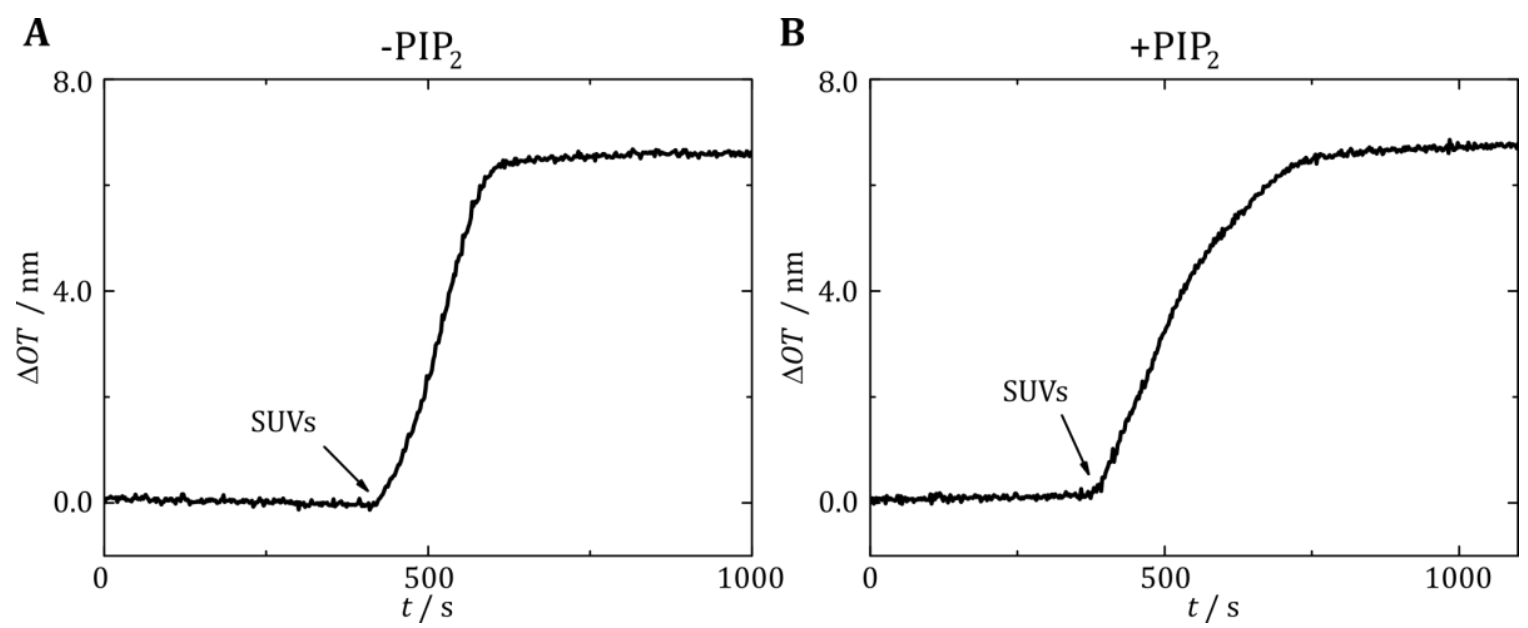

Figure 4.11: Exemplary time-trace of (A) POPC/POPS (80:20) and (B) POPC/POPS/PIP2 (75:20:5) vesicles spreading on hydrophilic silicon dioxide substrates.

The graphs in Figure 4.11 show that the formation of bilayers with both lipid composition were successful. The influence of the lipid head groups on the membrane height was then investigated using equation (4.3). Table 4.5 shows the calculated values of $\triangle O T_{\mathrm{M}}$ and $d$ of POPC/POPS (80:20) and POPC/POPS/PIP 2 bilayers.

Table 4.5: Mean values of the optical and physical thicknesses of the POPC/POPS (80:20) and POPC/POPS/PIP2 (75:20:5) lipid bilayers. The errors were given as standard deviations. $n$ describes the amount of performed experiments.

\begin{tabular}{|c|c|c|c|}
\hline Lipid composition & $\Delta O T_{\mathrm{M}} / \mathrm{nm}$ & $d / \mathrm{nm}$ & $n$ \\
\hline POPC/POPS (80:20) & $6.4 \pm 0.3$ & $4.3 \pm 0.2$ & 6 \\
\hline POPC/POPS/PIP2 (75:20:5) & $6.3 \pm 0.2$ & $4.2 \pm 0.1$ & 13 \\
\hline
\end{tabular}

Like shown in Table 4.5, increasing of the POPS content did not change the membrane thickness (cf. Table 4.1). There was also no difference in the POPC/POPS bilayer thickness containing or lacking PIP2. These results show that the negative charges of POPS and $\mathrm{PIP}_{2}$ changed the curve shape during the bilayer formation but did not influence the membrane thickness. The lateral diffusion of TxR in the POPC/POPS and POPC/POPS/PIP 2 bilayers was measured to be $D=1.9 \pm 0.3 \mu \mathrm{m}^{2} / \mathrm{s}$ (without $\mathrm{PIP}_{2}$ ) and $D=1.4 \pm 0.4 \mu \mathrm{m}^{2} / \mathrm{s}$ (with $\mathrm{PIP}_{2}$ ). Although the diffusion coefficient of $\mathrm{TxR}$ in the POPC/POPS/PIP2 bilayers slightly decreased compared to POPC/POPS and POPC/PIP2 bilayers $\left(1.8-2.5 \mu \mathrm{m}^{2} / \mathrm{s}\right)$, the difference got negligible within the deviations. 
Furthermore mobile fractions of $F_{\mathrm{m}}=86 \pm 8 \% \quad\left(\mathrm{POPC} / \mathrm{PIP}_{2}\right)$ and $F_{\mathrm{m}}=76 \pm 4 \%$ (POPC/POPS/PIP2) were determined. These results show that the formation of mobile bilayers composed of POPC/POPS and POPC/POPS/PIP2 was successful. Moreover; no obvious defects were detected by fluorescence microscopy (Figure 4.12).

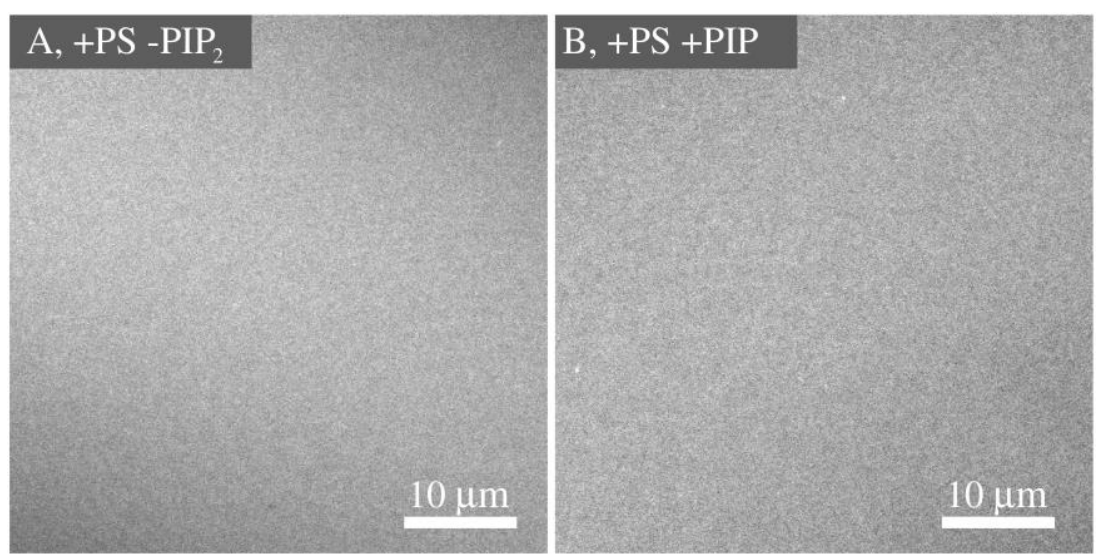

Figure 4.12: Fluorescence images of (A) POPC/POPS/TxR (79.5:20:0.5) and (B) POPC/POPS/PIP $2 / T x R$ (74.9:20:5:0.5) bilayers on silicon dioxide, showing a homogenous distribution of the fluorophore.

$\mathrm{PIP}_{2}$ is the receptor lipid for ENTH, has a negative charge of - 3 to -5 under physiological conditions ${ }^{44}$ and is binding in an ENTH binding pocket surrounded by positively charged amino acids. Moreover, previous studies on the ENTH-PIP2 interaction on various membrane models obtained higher binding affinity of ENTH to $\mathrm{PIP}_{2}$ when lipids with the head group PS (negatively charged) are involved.8,26 Therefore the next chapters focus on the influence of PS on the ENTH-PIP2 interaction.

\subsubsection{Influence of PS on the binding affinity of ENTH to PIP2}

The influence of PS on the binding affinity of ENTH was examined with RIfS measurements. By addition of small unilamellar vesicles (SUVs) to hydrophilic silicon dioxide wafers, the SUVs spread on the surface. Thereby the optical thickness increased, indicating the successful formation of a bilayer (cf. Section 3.3.2). To investigate if ENTH also binds to the negatively charged lipid PS, ENTH was added to a POPC/POPS (80:20) bilayer lacking PIP2 (Figure 4.13). 


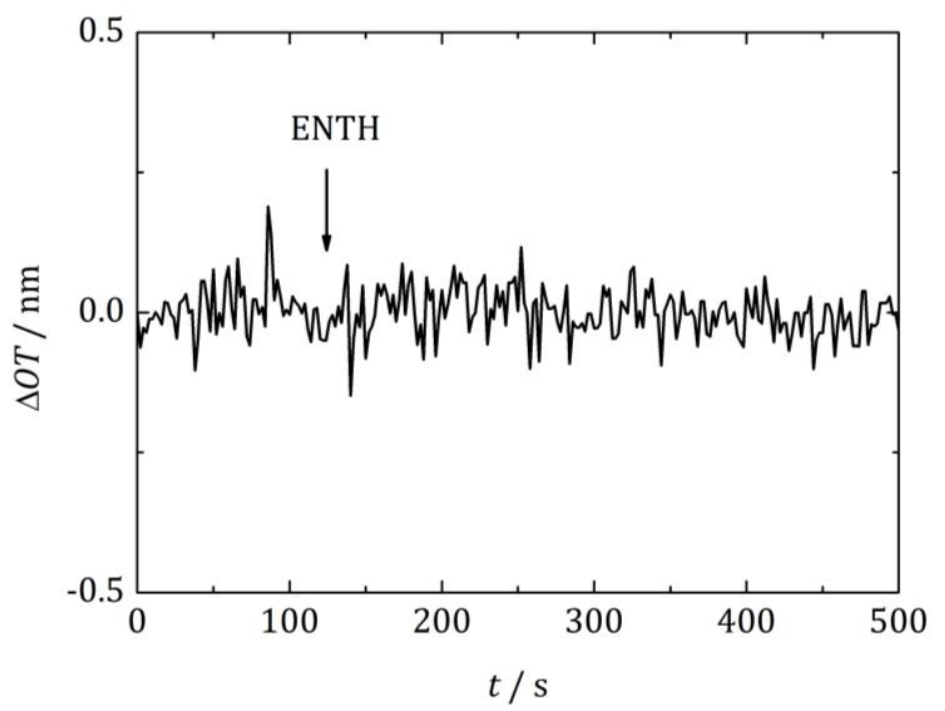

Figure 4.13: Exemplary time-trace of a RIfS blind experiment. Addition of ENTH $(1 \mu \mathrm{M})$ to a preformed POPC/POPS (80:20) bilayer did not show changes in the optical thickness, which proves that the ENTH binds specifically to $\mathrm{PIP}_{2}$ and does not interact with the negative charge of PS in the absence of $\mathrm{PIP}_{2}$.

No significant increase of $\triangle O T$ was observed when the ENTH domain was added to the POPC/POPS bilayer, showing no binding of ENTH to PS in the absence of PIP2. This proves the specific protein binding to its receptor lipid. Based on this, binding studies of ENTH to POPC/PIP2 (95:5) and POPC/POPS/PIP2 (75:20:5) bilayers were perfomed with RIfS experiments to investigate the influence of PS on the binding affinity of ENTH when $\mathrm{PIP}_{2}$ is present. In Figure 4.14 representative time traces upon addition of different ENTH concentrations to these bilayers are illustrated. 
A

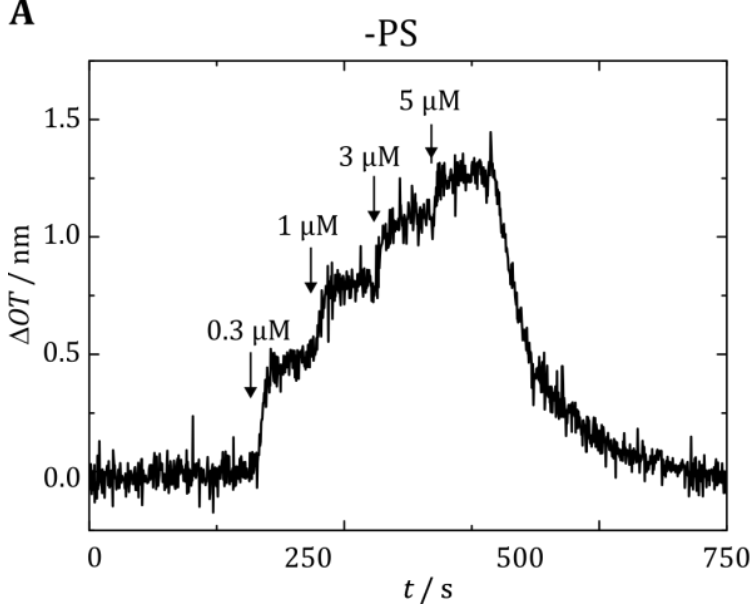

B

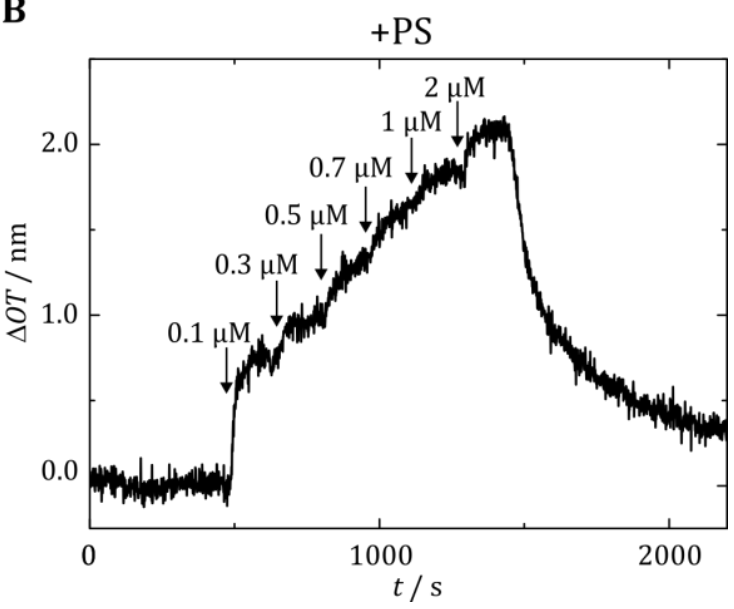

Figure 4.14: Representative RIfS time-traces of the ENTH adsorption upon different protein concentrations to POPC/PIP 2 bilayers in (A) absence and (B) presence of PS. The protein additions are marked by arrows. After rinsing with PBS the protein desorbed from the surface showing the reversible ENTH$\mathrm{PIP}_{2}$ binding. Lipid compositions: POPC/PIP2 (A, 95:5), POPC/POPS/PIP2 (B, 75:20:5).

For both lipid compositions the optical thickness increased with the addition of the protein domain. Higher ENTH concentrations led to increased $\triangle O T$ values. The difference between the experiments were the maximum $\triangle O T$ values, which reached values of $\triangle O T=1.3 \mathrm{~nm}$ at $5.0 \mu \mathrm{M}$ ENTH in absence and $\Delta O T=2.1 \mathrm{~nm}$ at $2.0 \mu \mathrm{M}$ ENTH in presence of PS, indicating a higher binding affinity of the protein to bilayers containing $\mathrm{PIP}_{2}$ and PS. With POPC/PIP 2 bilayers five and with POPC/POPS/PIP 2 bilayers eight individual experiments were performed. The isotherms revealed a high reversible binding of ENTH to POPC/PIP 2 (95:5). $96 \pm 10 \%$ of the ENTH desorbed from the membrane surface during rinsing with PBS. Compared to that $89 \pm 3 \%$ of the ENTH bound reversible to POPC/POPS/PIP2 (75:20:5) bilayers. A Mann-Whitney U test revealed that the differences are not significant $(p=0.2)$.

Based on this data the Langmuir adsorption model was used to calculate the dissociation constant $\left(K_{\mathrm{D}}\right)$ of the ENTH binding to $\mathrm{PIP}_{2}$-containing membranes as well as the maximum of the optical thickness after protein addition $\left(\triangle O T_{\mathrm{ENTH}}\right.$ max $)$. This model is in a good accordance with the used system, when the surface is homogenous, all binding sites are equal as well as a monolayer is formed and when no interactions of the adjacent molecules occur.101,102 Furthermore a dynamic equilibrium of the bound and free molecules is assumed. As the vesicle spreading was successful (cf. 4.1.1 and 4.2.1), indicating a homogenous membrane surface and the ENTH binding is almost 
completely reversible the used system seems to match these assumptions. In Figure 4.15 the adsorption isotherms of ENTH to POPC/PIP 2 (95:5) and POPC/POPS/PIP2 (75:20:5) bilayers are illustrated, showing the influence of the lipid composition on the binding affinity.

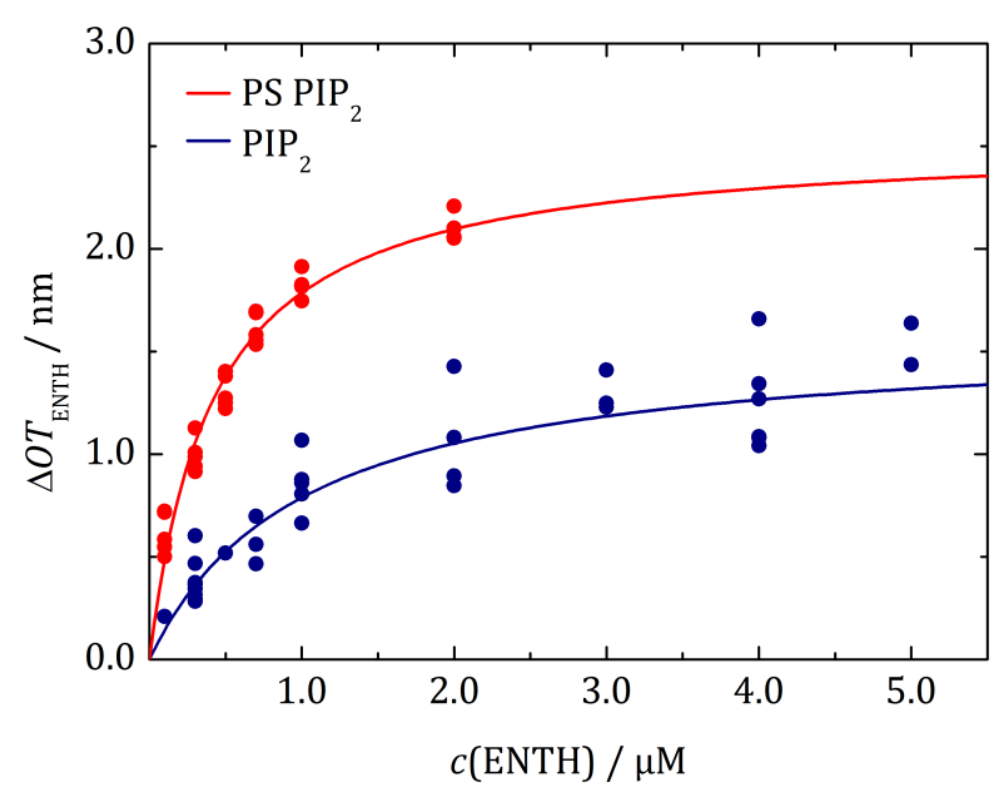

Figure 4.15: Adsorption isotherms of ENTH to POPC/PIP2 (95:5) (blue circles) and POPC/POPS/PIP2 (75:20:5) (red circles) bilayers. The values for $\triangle O T_{\mathrm{ENTH}}$ max and $K_{\mathrm{D}}$ were obtained by fitting a Langmuir adsorption isotherm (solid lines) to the data. Non-linear regression weighted by the corresponding number of measurements that went into each concentration was carried out using a Levenberg-Marquardt algorithm. ${ }^{103,104}$

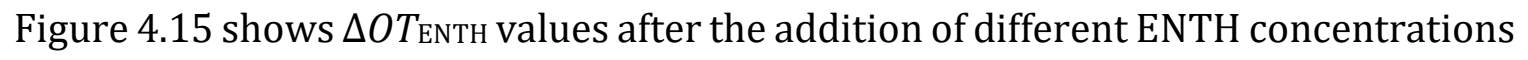
to POPC/PIP2 (blue circles) and POPC/POPS/PIP2 (red circles) bilayers.

The data was fitted with a Langmuir adsorption isotherm (solid lines). Due to different numbers of data points, a non-linear regression with a Levenberg-Marquardt algorithm weighted by the number of realized measurements for each concentration was carried out. ${ }^{103,104}$ This enabled the determination of the maximal change in optical thickness after protein addition $\triangle O T_{\mathrm{ENTH}}$ max and the dissociation constant $K_{\mathrm{D}}$ by equation (4.4). 


$$
\Delta O T=\Delta O T_{\mathrm{ENTH}, \max } \frac{c}{K_{\mathrm{D}}+c}
$$

When no PS was present, mean values of $\Delta O T_{\mathrm{ENTH}, \max }=1.6 \pm 0.1 \mathrm{~nm}$ and $K_{\mathrm{D}}=1.0 \pm 0.2 \mu \mathrm{M}$ were obtained. For the POPC/POPS/PIP 2 system the mean values of $\Delta O T_{\mathrm{ENTH}, \max }=2.5 \pm 0.1 \mathrm{~nm}$ and $K_{\mathrm{D}}=0.42 \pm 0.05 \mu \mathrm{M}$ were calculated, indicating the higher binding affinity of ENTH to bilayers containing POPS in the presence of PIP2.

\subsubsection{Topographical analysis of ENTH cluster formation}

In the previous chapters the specific binding of ENTH to PIP2 containing bilayers was shown. The topography of the substrate surface before and after protein binding was investigated by means of atomic force microscopy (AFM). This technique enables to obtain information about the protein organization on supported membrane models. ${ }^{105,106}$ The RIfS measurements showed an increased binding affinity and a higher occupancy of ENTH when lipids with the head group PS were present in the bilayer (cf. chapter 4.2.2). Therefore the surface topographies of $\mathrm{PIP}_{2}$ doped supported bilayers with and without PS were analyzed. For better comparison with previous results, $^{26,79}$ AFM experiments were performed with bilayers composed of DOPC/DOPE/PIP2/TxR (64.9:30:5:0.1) and DOPC/DOPE/DOPS/PIP2/TxR (44.9:30:20:5:0.1). Fluorescently labeled supported lipid bilayers were generated as described in chapter 3.2.2. Afterwards force-distance curves were recorded via AFM, which enabled to determine the breakthrough force and the membrane thicknesses (cf. chapter 3.3.4). The breakthrough force is the maximum vertical force at which the bilayer is able to stand before the cantilever tip breaks through the membrane. ${ }^{107}$ In Figure 4.16 an exemplary force distance curve with a breakthrough event is illustrated. 


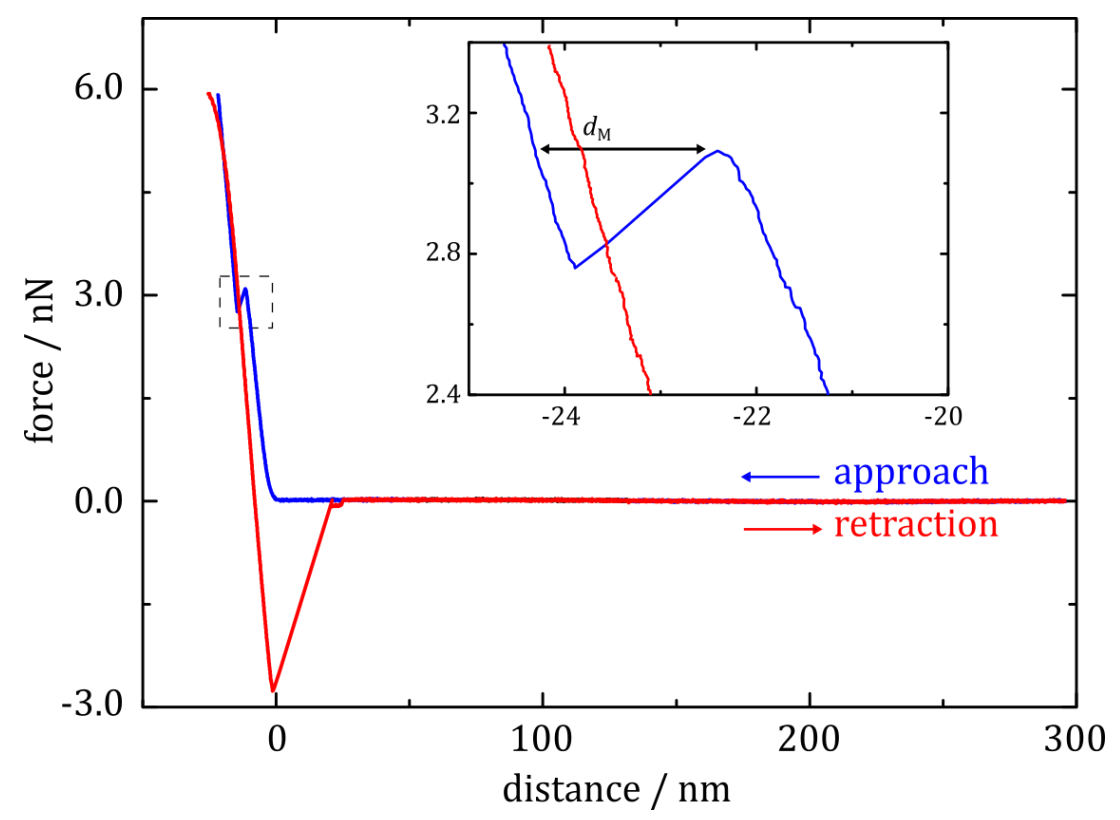

Figure 4.16: A representative force-distance curve measured on DOPC/DOPE/DOPS/PIP $/ \mathrm{P}_{2}$ (44.9:30:20:5:0.1).The inlet shows the breakthrough event (dashed square) which enabled to determine the breakthrough force and the membrane thickness $d_{\mathrm{M}}$

Breakthrough forces were measured to be between 2-6 nN. Furthermore the AFM experiments revealed lipid bilayers with physical thicknesses of $d=3.9 \pm 0.8 \mathrm{~nm}$ (without PS) and $d=3.7 \pm 07 \mathrm{~nm}$ (with PS). Also the FRAP experiments proved the formation of mobile bilayers. After bleaching a region of interest (ROI) the fluorescence recovered in the ROI. Diffusion coefficients of $2.5 \pm 0.6 \mu \mathrm{m}^{2} / \mathrm{s}$ were measured for TxR in the DOPC/DOPE/PIP $/ T x R \quad(64.9: 30: 5: 0.1)$ and $1.7 \pm 0.4 \mu \mathrm{m}^{2} / \mathrm{s}$ in the DOPC/DOPE/DOPS/PIP2/TxR (44.9:30:20:5:0.1) bilayers on hydrophilic silicon dioxide using a MATLAB Script by Jönsson et al. ${ }^{78}$ The mobile fraction of TxR in the bilayers without PS were determined to be $83 \pm 3 \%$ and the value for bilayers with PS resulted in a mobile fraction of $71 \pm 5 \%$. Hence the AFM and FRAP experiments proved the formation and mobility of the bilayers.

When the bilayer formation was successful the surface was imaged by AFM using contact mode (cf. chapter 3.3.4). This enabled to compare the surface topography before and after protein addition. Then ENTH $(1 \mu \mathrm{M})$ was added and the solution was mixed by stirring for $2 \mathrm{~h}$. Subsequently again images of the surface were taken. The atomic force micrographs before and after ENTH incubation are shown in Figure 4.17. 

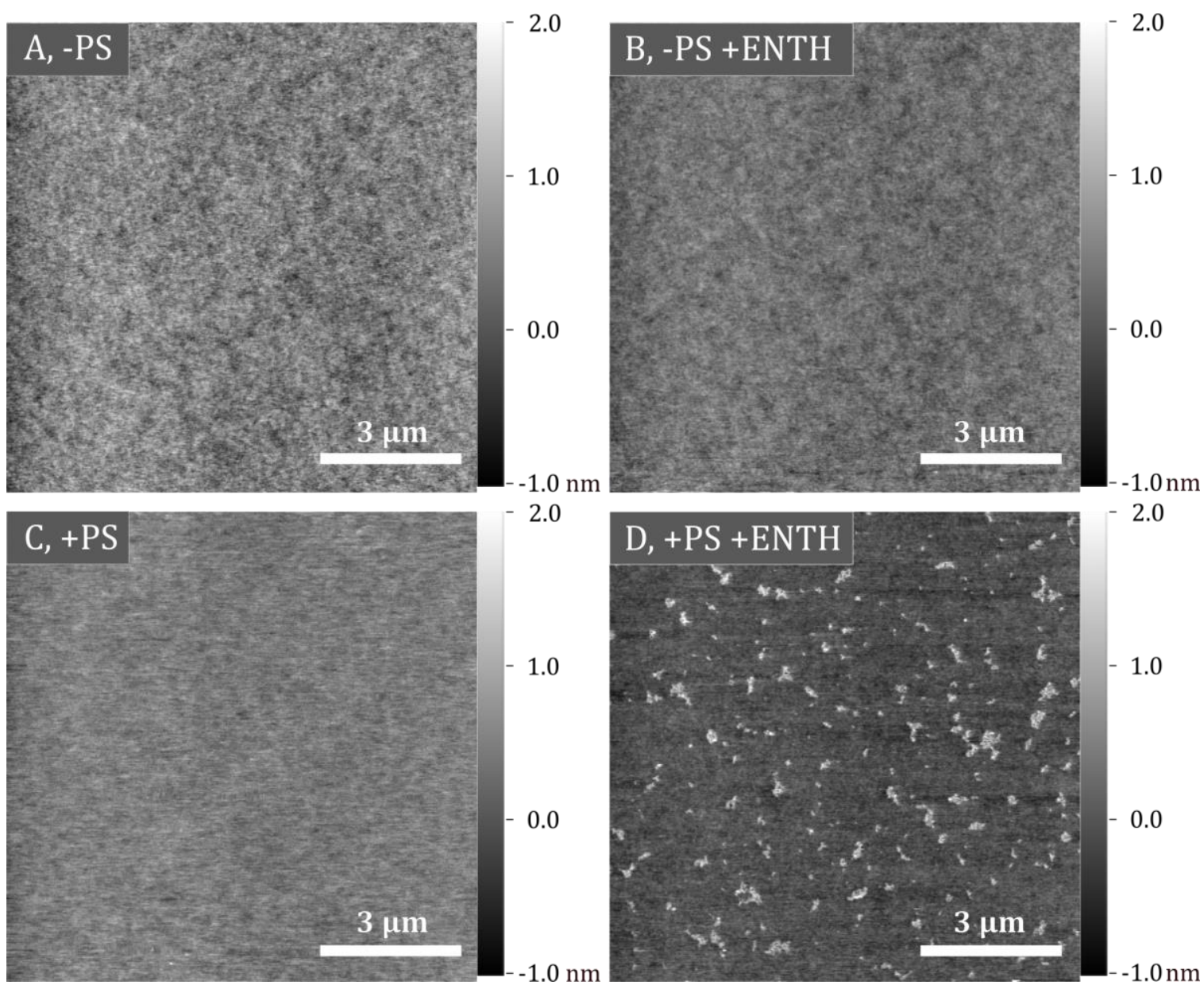

Figure 4.17: Atomic force micrographs of (A) DOPC/DOPE/PIP $/$ /TxR (64.9:30:5:0.1) and (C) DOPC/DOPE/DOPS/PIP2/TxR (44.9:30:20:0.1) bilayers on hydrophilic silicon dioxide wafers prior ENTH addition. (B) and (D) show the corresponding micrographs after $2 \mathrm{~h}$ of ENTH incubation $(1 \mu \mathrm{M})$. Only in the presence of PS, protein clusters were observed on the membrane surface.

The micrographs show that before ENTH addition the DOPC/DOPE/PIP2/TxR (Figure 4.17 A) and DOPC/DOPE/DOPS/PIP2/TxR (Figure 4.17 C) bilayers had a homogenous surface with roughnesses of $\mathrm{rms}=174 \pm 17 \mathrm{pm} \quad(n=7)$ without PS and $\mathrm{rms}=158 \pm 14 \mathrm{pm}(n=5)$ with PS.

Addition of ENTH to the DOPC/DOPE/PIP $/$ TxR bilayers (Figure $4.17 \mathrm{~B}$ ) led to a roughness of rms $=196 \pm 22 \mathrm{pm}(n=21)$, but no structures were observed (Figure 4.17 C). When PS was present in the bilayers incubation with ENTH for $2 \mathrm{~h}$ caused the appearance of small structures, which were about $0.5 \mu \mathrm{m}$ larger or smaller. To investigate if these structures could be assigned to protein adsorption the heights of these clusters were analyzed using a MatLab Script written by Dr. Ingo Mey (Georg-August- 
University Göttingen). A threshold of the membrane height enabled to distinguish between membrane and adsorbed molecules, so that the protein occupancy and height could be determined (cf. chapter 3.3.4). From the AFM images the protein occupancy of $6 \pm 1 \%$ and the protein height of $1.2 \pm 0.5 \mathrm{~nm}$ (mean value $\pm \mathrm{SD}, n=32$, with $n$ the number of evaluated images from three independent experiments) were calculated (Figure 4.18).

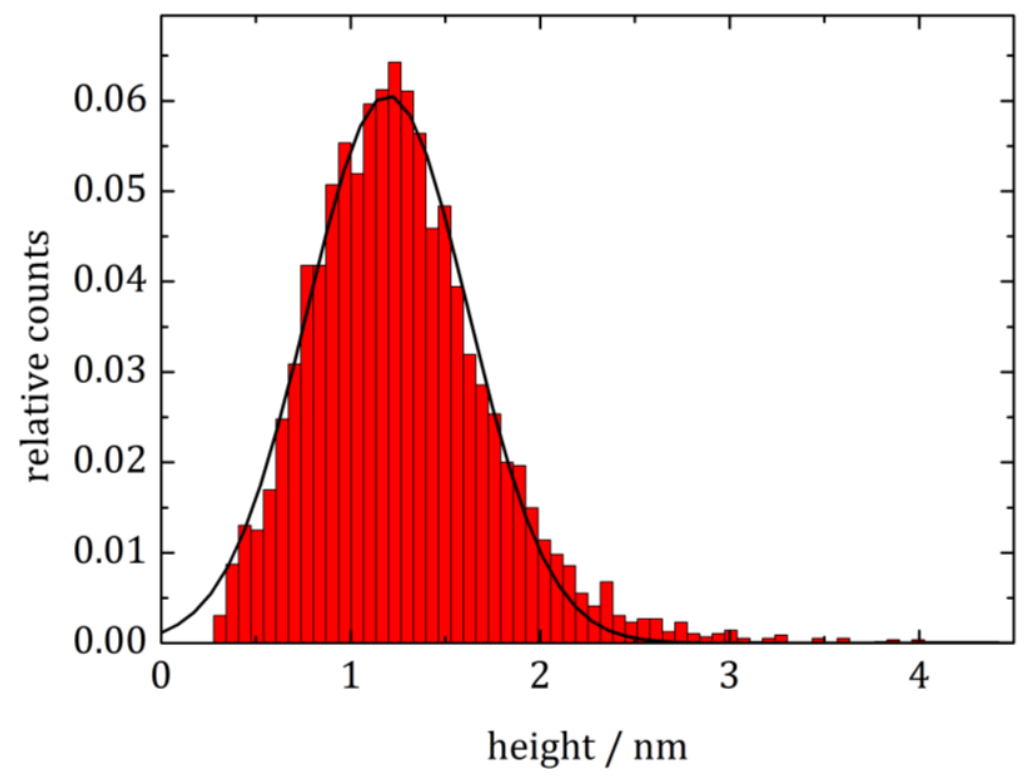

Figure 4.18: Histogram plot of the protein heights averaged over 32 micrographs. A protein height of $1.2 \pm 0.5 \mathrm{~nm}$ was determined by fitting a normal distribution. The bin size $(0.066 \mathrm{~nm})$ was calculated according to Freedman et al. ${ }^{108}$

With these results it seemed likely that the small structures were caused by protein adsorption and were attributed to ENTH clusters. Due to the ENTH cluster formation the surface roughness increased up to $\mathrm{rms}=263 \pm 33 \mathrm{pm}(n=32)$.

To ensure that the protein really bound to the membrane and not only to defects, occurring in the membrane, fluorescence images of these bilayers before and after ENTH incubation were taken. As control also the surface of membranes without ENTH incubation were imaged after $2 \mathrm{~h}$, showing a homogenous fluorescence over the whole substrates without visible defects in all cases (Figure 4.19). The few small bright spots were attributed to SUVs adhered to the surface. 


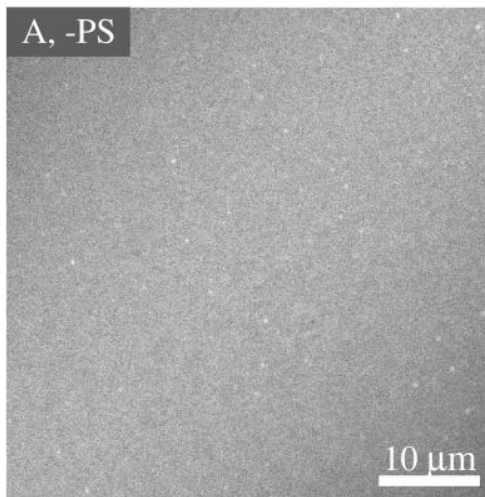

$\mathrm{B},+\mathrm{PS}$

\section{$\mathrm{B},+\mathrm{PS}$}

\section{A1, -PS -ENTH}

$10 u \mathrm{~m}$

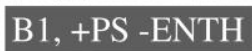

A2, - PS +ENTH

$10 \mu \mathrm{m}$

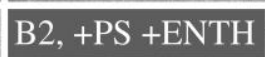

Figure 4.19: Fluorescence images of (A) DOPC/DOPE/PIP $/$ TxR (64.9:30:5:0.1) and (B) DOPC/DOPE/DOPS/PIP2/TxR (44.9:30:20:5:0.1) bilayers. After 2h of incubation without (A1, B1) and with $1 \mu \mathrm{M}$ ENTH $(\mathrm{A} 2, \mathrm{~B} 2)$ the bilayers were imaged again. In all cases a homogenous distribution of TxR was observed.

AFM allows imaging in a nanometer range resolution ${ }^{109}$ so even single protein adsorption should be possible to observe. The discrepancy of the protein coverage in RIfS (cf. chapter 4.2.2) and AFM is not obvious. Hence FRAP measurements were performed to analyze the mobility of the bilayers after the ENTH incubation. The diffusion coefficients of DOPC/DOPE/PIP $/$ TxR $\quad(64.9: 30: 5: 0.1)$ and DOPC/DOPE/DOPS/PIP2/TxR (44.9:30:20:5:0.1) bilayers after ENTH addition were determined. The bilayers were still mobile, but the mobility was decreased. In Table 4.6 the diffusion coefficients of TxR in the bilayers before and after ENTH addition are listed. 
Table 4.6: Diffusion coefficients of TxR in DOPC/DOPE/PIP $/$ TxR (64.9:30:5:0.1) and DOPC/DOPE/DOPS/PIP $/$ TxR (44.9:30:20:5:0.1) bilayers before and after the incubation of $1 \mu \mathrm{M}$ ENTH.

\begin{tabular}{|c|c|c|}
\hline lipid composition & before ENTH addition & after ENTH addition \\
\hline $\begin{array}{l}\text { DOPC/DOPE/PIP } / \text { TxR } \\
(64.9: 30: 5: 0.1)\end{array}$ & $2.5 \pm 0.6 \mu \mathrm{m}^{2} / \mathrm{s}$ & $0.6 \pm 0.2 \mu \mathrm{m} / \mathrm{s}^{2}$ \\
\hline $\begin{array}{l}\text { DOPC/DOPE/DOPS/PIP } / \text { TxR } \\
(44.9: 30: 20: 5: 0.1)\end{array}$ & $1.7 \pm 0.4 \mu \mathrm{m}^{2} / \mathrm{s}$ & $0.3 \pm 0.2 \mu \mathrm{m} / \mathrm{s}^{2}$ \\
\hline
\end{tabular}

The results show that the diffusion coefficient of TxR in the PS containing membranes after ENTH incubation was about the half of the diffusion coefficient of TxR in the DOPC/DOPE/PIP 2 bilayers when ENTH was present. Although the protein adsorption seemed to modulate the membrane mobility, imaging of single protein molecules was prevented due to a dynamic protein distribution in contrast to more immobile ENTH clusters (when PS is present).

\subsubsection{Investigation of the ENTH R114A mutant binding to PIP2}

The ENTH domain consists of 150 amino acids, which are essential for various intraand intermolecular interactions. Mutation experiments were done by Ford et al. to investigate the role of the amphipathic helix in the membrane deformation process. ${ }^{21}$ With co-sedimentations assays they presented that the binding affinity and membrane deformation activity change when the sixth amino acid in the helix was mutated. The binding affinity decreased with hydrophilicity of the amino acid residue. Furthermore, Yoon et al. used electron paramagnetic resonance (EPR) and fluorescence microscopy showing that clustering of the ENTH domain appears. ${ }^{28}$ With the mutants K23E and E42K only binding, but no clustering of ENTH was observed, showing the importance of these amino acids for protein-protein interactions. Lai et al. presented atomistic MD simulations, where they identified three important ENTHmembrane interaction regions: The binding pocket for $\mathrm{PIP}_{2}$, the $\alpha 0$-helix and the R114 loop (cf. Figure 1.3). ${ }^{110}$ 
In order to specify the regions of the ENTH domain involved in certain interactions, experiments with the ENTH R114A mutant were performed. In this mutant the arginine in position 114 in the loop between the sixth and seventh helix is exchanged by an alanine. The mutant was expressed and purified by Dr. Ben Kroppen and Indrani Mukherjee from the group of Prof. Dr. Michael Meinecke (Department of Cellular Biochemistry, University Medical Center Göttingen). First of all, RIfS measurements were done to investigate the binding behavior of the mutant depending on the lipid composition. Therefore vesicles composed of POPC/PIP 2 (95:5) and POPC/POPS/PIP2 (75:20:5) were added to hydrophilic silicon dioxide substrates (cf. chapter 3.3.1), resulting in vesicle spreading and the formation of a bilayer. After rinsing with PBS and passivation with BSA, the ENTH R114A mutant was added. The ENTH concentration was increased stepwise (Figure 4.20, indicated by arrows). A representative time-traces of the optical thickness $(\triangle O T)$ after ENTH R114A addition to POPC/PIP 2 and POPC/POPS/PIP 2 bilayers are plotted in Figure 4.20.

A

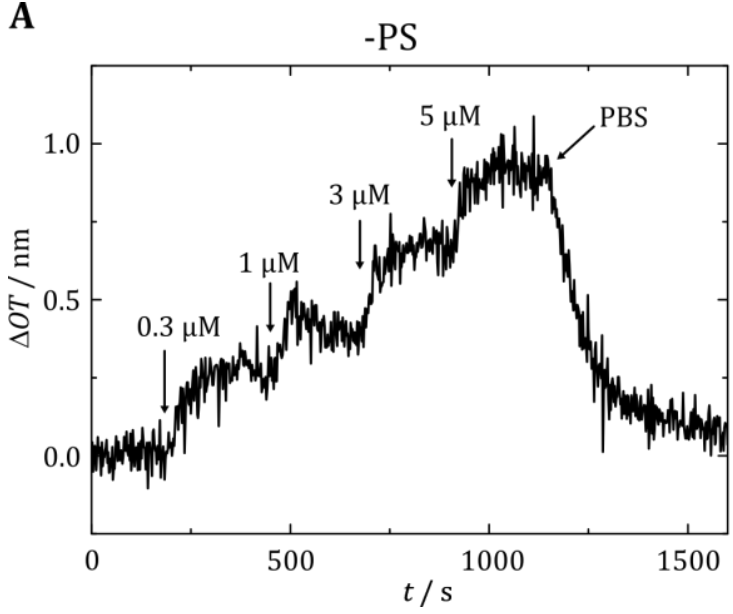

B

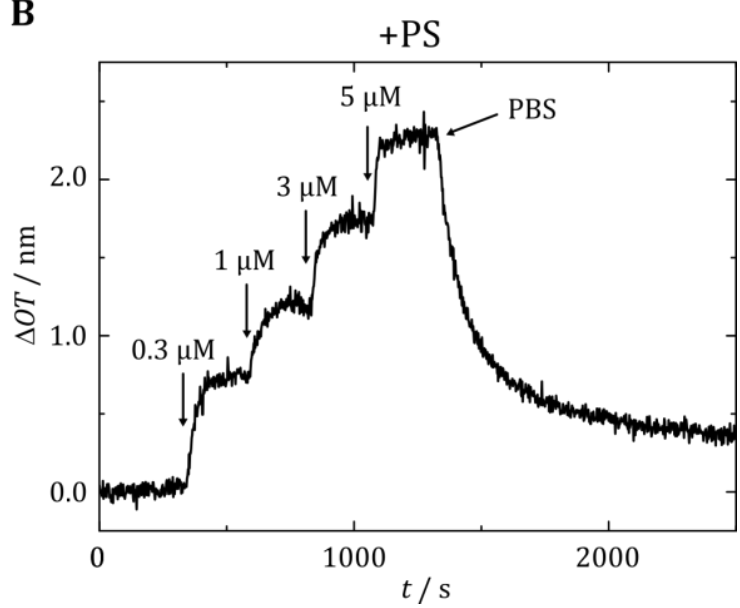

Figure 4.20: Time-resolved change in optical thickness upon addition of different ENTH R114A concentrations (marked by arrows) to (A) POPC/PIP 2 (95:5) and (B) POPC/POPS/PIP 2 (75:20:5) bilayers. The protein desorbs after rinsing with PBS showing the almost complete reversibility of the ENTH R114A binding.

In both cases the optical thickness increased by ENTH R114A addition until it reached a plateau. With every further and higher concentrated protein addition the optical thickness was stepwise increased due to higher protein occupancy on the surface. Rinsing with PBS led to desorption, which indicated the reversible binding. When the 
bilayer was lacking POPS the $\triangle O T$ reached a value of $0.9 \mathrm{~nm}$ at an ENTH R114A concentration of $5.0 \mu \mathrm{M}$ (Figure $4.20 \mathrm{~A}$ ). In case of bilayers with $20 \mathrm{~mol} \%$ PS the $\triangle O T$ increased up to $2.3 \mathrm{~nm}$ when $5.0 \mu \mathrm{M}$ ENTH R114A was added (Figure $4.20 \mathrm{~B}$ ). This indicated a higher amount of bound mutant when PS is present. To obtain the maximum change in optical thickness and the binding affinity of the mutant to POPC/PIP2 and

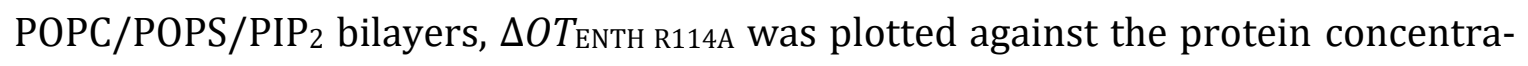
tion (Figure 4.21).

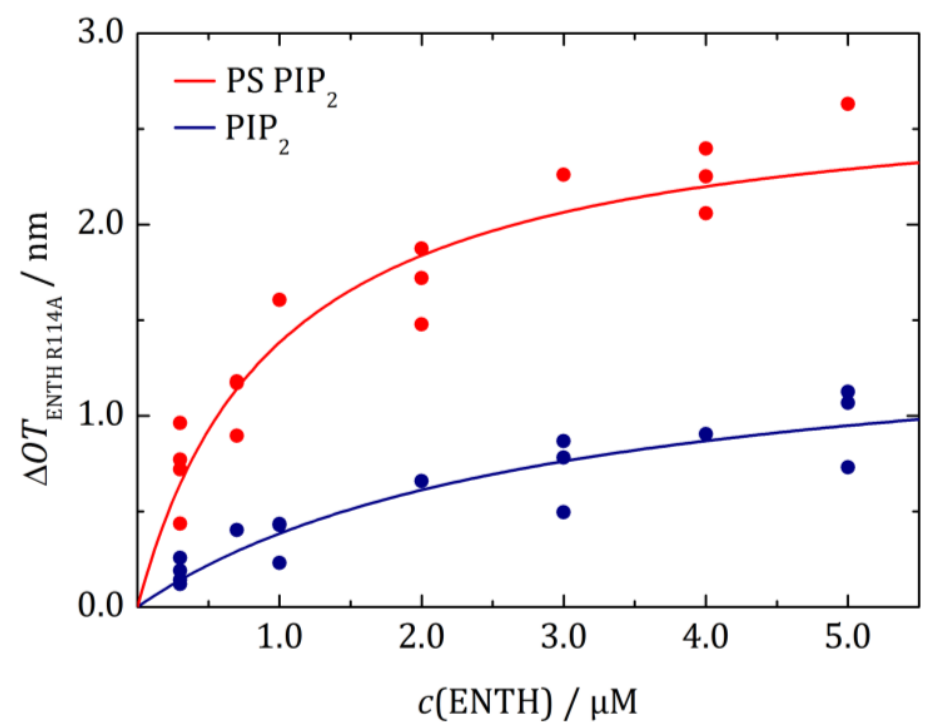

Figure 4.21: Adsorption isotherms of ENTH R114A to POPC/PIP2 (95:5) (blue circles) and POPC/POPS/PIP2 (75:20:5) (red circles) bilayers. The values for $\Delta \Delta O T_{\max }$ and $K_{\mathrm{D}}$ (Tab. 1) were obtained by fitting a Langmuir adsorption isotherm (solid lines) to the data. Non-linear regression weighted by the corresponding number of measurements that went into each concentration was carried out using a Levenberg-Marquardt algorithm. ${ }^{103,104}$

Figure 4.21 shows the changes in optical thicknesses upon addition of different ENTH R114A concentrations to POPC/PIP 2 (blue circles) and POPC/POPS/PIP 2 (red circles) bilayers. Four individual measurements without POPS and four with POPS at protein concentrations of 0.3 to $5.0 \mu \mathrm{M}$ were performed. By fitting the data with a Langmuir adsorption isotherm the maximum of the optical thickness change $\triangle O T_{\text {ENTH R114A,max }}$ and the dissociation constant $K_{\mathrm{D}}$ were obtained, showing the higher binding affinity of the ENTH R114 mutant to PS containing lipid bilayers. A non-linear regression was done using a Levenberg-Marquardt algorithm. ${ }^{103,104}$ Thereby the regression was weighted by the amount of measurements for each concentration. For the POPC/PIP2 
(95:5) system a $K$ D of $2.9 \pm 0.8 \mu \mathrm{M}$ and a $\Delta O T_{\text {ENTH R114A, } \max }=1.5 \pm 0.2 \mathrm{~nm}$ were determined. Bilayers composed of POPC/POPS/PIP2 (75:20:5) exhibited a three times smaller $K$ D value of $1.0 \pm 0.3 \mu \mathrm{M}$ and $\Delta O T_{\text {ENTH R114A, max }}$ of $2.7 \pm 0.2 \mathrm{~nm}$, which is twice

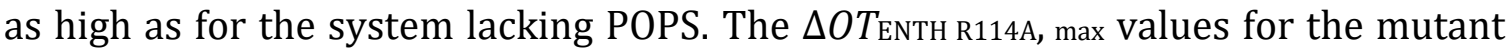
are similar to those of the ENTH wildtype (wt), but the binding affinity of the mutant to both lipid systems decreased compared to that of ENTH wt. All values for the ENTH wt and the mutant are summarized in Table 4.7.

Table 4.7: Summary of the fit results of the Langmuir adsorption isotherms for ENTH wt and ENTH $\mathrm{R} 114 \mathrm{~A}$. The $K_{\mathrm{D}}$ and the $\triangle O T_{\mathrm{ENTH}}$ max are given as parameter $\pm \mathrm{SE}$.

\begin{tabular}{|l|l|l|l|l|}
\hline \multirow{2}{*}{$\begin{array}{l}\text { lipid } \\
\text { composition }\end{array}$} & \multicolumn{3}{|c|}{$K_{\mathrm{D}} / \mu \mathrm{M}$} & \multicolumn{2}{c|}{$\Delta O T_{\text {ENTH, max }} / \mathrm{nm}$} \\
\cline { 2 - 5 } & ENTH wt & ENTH R114A & ENTH wt & ENTH R114A \\
\hline $\begin{array}{l}\text { POPC/ PIP } 2 \\
(95: 5)\end{array}$ & $1.0 \pm 0.2$ & $2.9 \pm 0.8$ & $1.6 \pm 0.1$ & $1.5 \pm 0.2$ \\
\hline $\begin{array}{l}\text { POPC/POPS/PIP2 } \\
(75: 20: 5)\end{array}$ & $0.42 \pm 0.05$ & $1.0 \pm 0.3$ & $2.5 \pm 0.1$ & $2.7 \pm 0.2$ \\
\hline
\end{tabular}

Table 4.7 shows that the mutant was still binding to the $\mathrm{PIP}_{2}$-containing membranes, but its binding affinity to $\mathrm{PIP}_{2}$ doped membranes changed. The mutant also bound reversible to those membranes. For the POPC/PIP 2 (95:5) bilayers a reversibility of $84 \pm 9 \%$ was determined. Moreover $89 \pm 3 \%$ of the ENTH R114 mutant bound reversibly to POPC/POPS/PIP2 (75:20:5) bilayers.

Furthermore ENTH wt formed clusters on bilayers composed of DOPC/DOPE/DOPS/PIP2/TxR, which was shown by AFM experiments (cf. Figure 4.17). To compare if also ENTH R114A mutant clusters on PS containing supported bilayers appear, atomic force micrographs of the surface before and after protein addition were taken. In Figure 4.22 the micrographs of DOPC/DOPE/PIP $/$ TxR (64.9:30:5:0.1) and DOPC/DOPE/DOPS/PIP2/TxR (44.9:30:20:0.1) bilayers before $(\mathrm{A}, \mathrm{C})$ and after (B, D) ENTH addition are illustrated. 

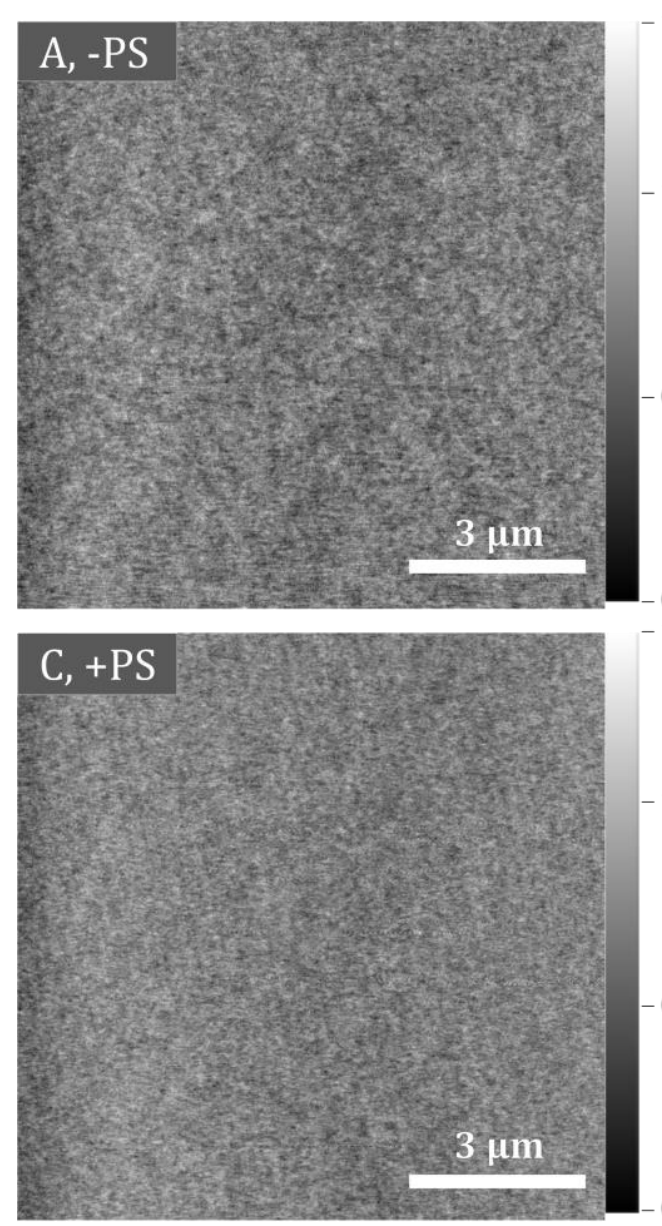
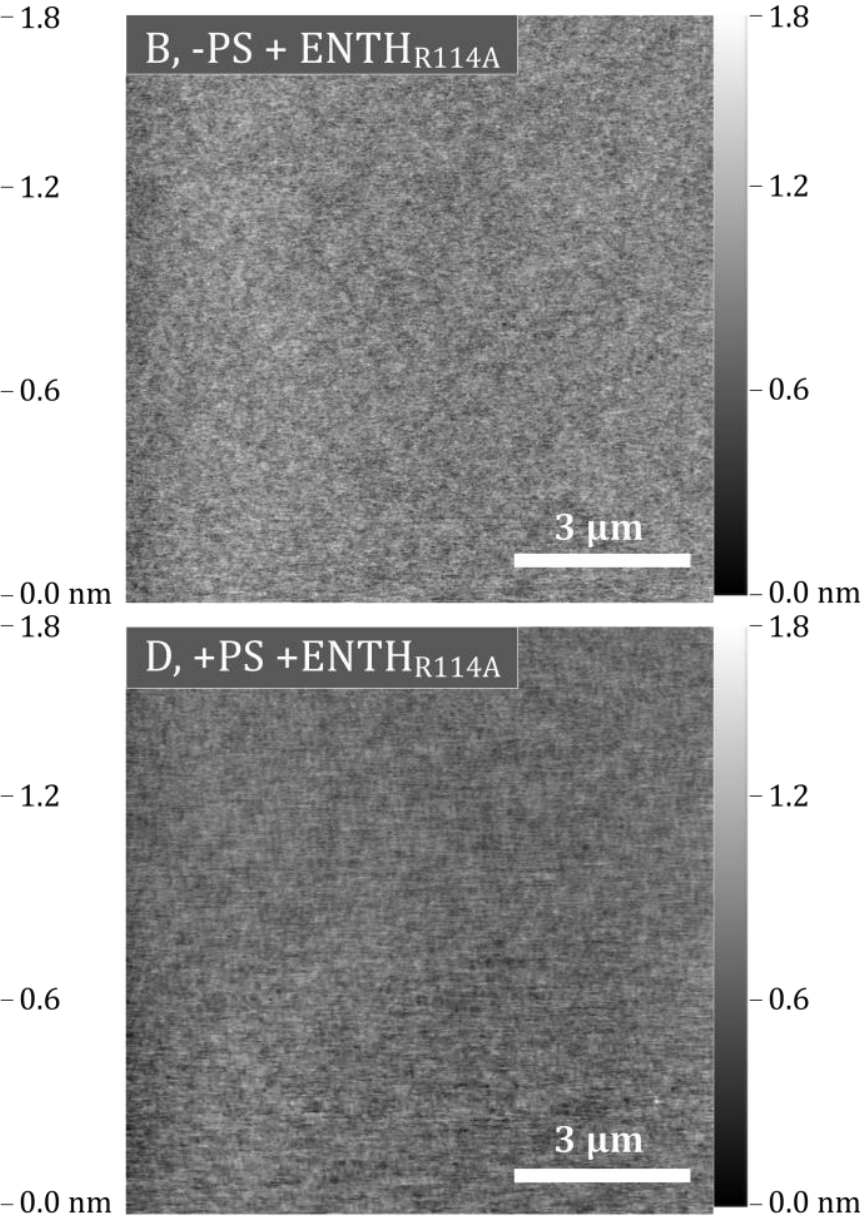

Figure 4.22: Atomic force micrographs of (A) DOPC/DOPE/PIP $2 / T x R(64.9 / 30 / 5 / 0.1)$ and (C) DOPC/DOPE/DOPS/PIP $/$ TxR (44.9/30/20/0.1) bilayers on hydrophilic silicon dioxide wafers prior protein addition. The corresponding micrographs (B, D) were obtained after $2 \mathrm{~h}$ of ENTH R114A incubation $(1 \mu \mathrm{M})$. Even in the presence of DOPS, no protein clusters were observed on the membrane surface indicating the necessity of the amino acid R114 for ENTH cluster formation.

The surfaces of the DOPC/DOPE/PIP2/TxR (Figure 4.22 A) and DOPC/DOPE/DOPS/PIP2/TxR (Figure 4.22 C) bilayers showed a homogenous distribution of the membrane. For the bilayers without PS a surface roughness of rms $=142 \pm 13$ pm $(n=3)$ was determined. When PS-containing bilayers were imaged surface roughness of rms $=142 \pm 12 \mathrm{pm}(n=4)$ was obtained. The micrographs also showed that in both cases no specific structures were observed on the surface after the protein addition (Figure 4.22 B and D) in contrast to the ENTH wt (cf. Figure 4.17). The roughness of the surfaces after ENTH incubation were calculated to be slightly higher with $\mathrm{rms}=152 \pm 7 \mathrm{pm}(n=3)$ lacking PS and $\mathrm{rms}=197 \pm 41 \mathrm{pm}$ $(n=11)$ in presence of PS. This showed that the surface nature changed due to ENTH 
binding, although no ENTH R114A clusters were observed, indicating that the amino acid R114A is important for clustering of ENTH.

\subsection{Investigation of the ENTH-PIP 2 interactions on lipid monolay- ers}

The concentration of the receptor lipid $\mathrm{PIP}_{2}$ and the presence of PS have an influence on the amount of bound ENTH (cf. sections 4.1.3 and 4.2.2). Moreover, it was shown that substrate interactions with the membrane lead to asymmetric artificial lipid bilayers on hydrophilic silicon dioxide surfaces. This was shown by binding studies on supported lipid monolayers (cf. chapter 4.1.3), indicating a higher protein occupancy compared to supported bilayers.

Another possibility to investigate the interactions of ENTH with monolayers is the Langmuir-Blodgett trough with the advantage that interactions with substrate surfaces are prevented. This method allowed to investigate the surface activity of ENTH as a function of the lipid composition. By measuring surface pressure changes upon ENTH addition, the penetration ability of the ENTH helix could be determined. The influence of the lipids $\mathrm{PIP}_{2}$ and PS on the ENTH penetration ability was investigated. First of all, surface pressure-area isotherms of all lipid compositions were recorded to analyze how the lipid composition influences the monolayer. Moreover, it was important to analyze the monolayer stability of the desired lipid mixtures. Only if the monolayers were stable enough over the experimental time, meaning lipids did not submerge into the subphase, it was guaranteed that enough $\mathrm{PIP}_{2}$ molecules were available for ENTH binding. Finally, penetration experiments with different lipid mixtures were performed.

\subsection{1 $\mathrm{PIP}_{2}$ influence on the ENTH penetration ability}

Binding of ENTH to bi- and monolayers is dependent on the concentration of the receptor lipid PIP2 (cf. chapter 4.1.3). Due to this the intermolecular interactions of the 
lipids within these membrane models are of great interest. Depending on the head groups, the number of carbon atoms in the fatty acid chains and their conformation, the isotherms can differ. ${ }^{111,112}$ These isotherms help to understand the lateral intermolecular interactions in the monolayer and the influence on protein-membrane interactions. The Langmuir-Blodgett trough technique was used to record pressurearea isotherms of POPC/POPE monolayers with different PIP2 content. To mimic the natural plasma membrane most closely POPC and POPE were used as these are present in membranes in a high amount. ${ }^{56}$ Furthermore, $\mathrm{PIP}_{2}$ was added as receptor lipid for the ENTH domain. Isotherms of POPC/POPE (80:20) and POPC/POPE/PIP2 (80$\mathrm{x}: 20$ : $\mathrm{x}$, with $\mathrm{x}=1,5,10 \mathrm{~mol} \%$ ) were measured in ultrapure water. As the following penetration experiments with protein were performed in HEPES buffer, also isotherms were recorded in HEPES buffer. The corresponding averaged surface pressure $(\pi)$ versus area per molecule $\left(A_{\mathrm{M}}\right)$ diagrams are plotted in Figure 4.23.
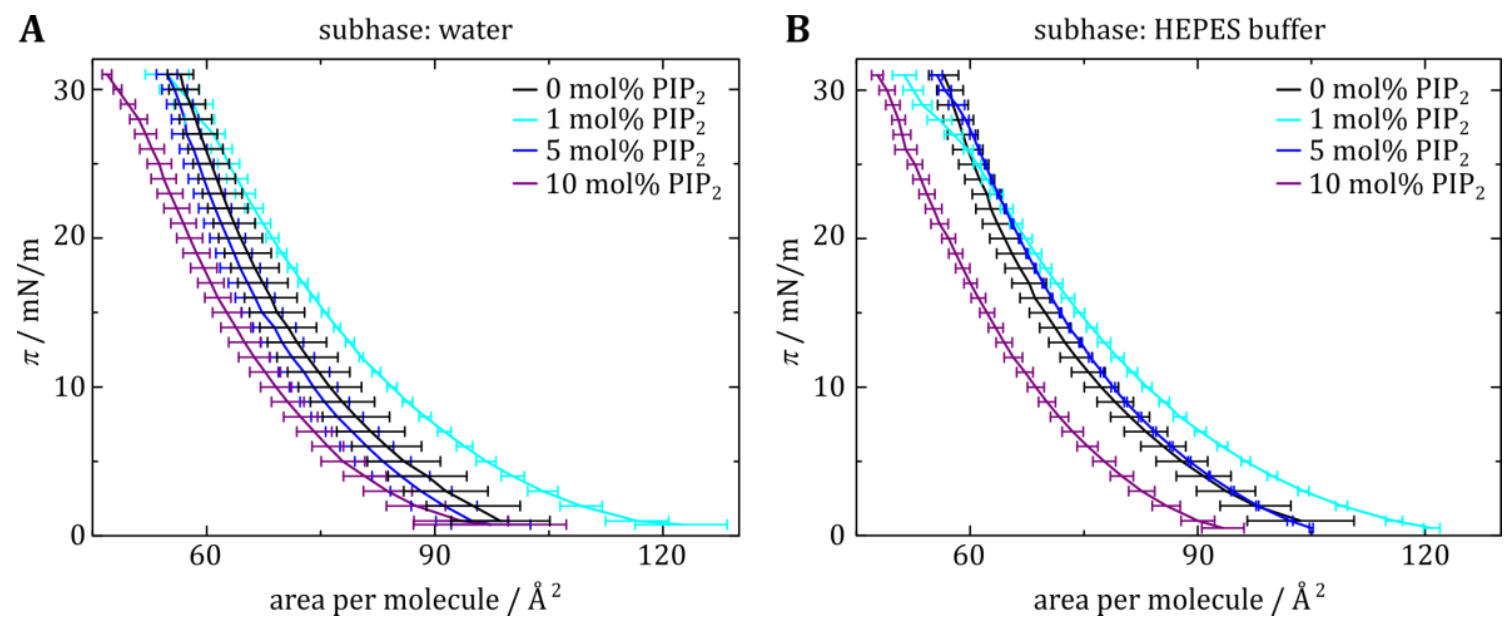

Figure 4.23: Averaged surface pressure-area per molecule isotherms of POPC/POPE (80:20, black), POPC/POPE/PIP 2 (79:20:1, light blue), POPC/POPE/PIP 2 (75:20:5, blue) and POPC/POPE/PIP 2 (70:20:10, purple) at $20^{\circ} \mathrm{C}$. The subphases were ultrapure water (A) and HEPES buffer (B). The error bars represents the standard deviations of the area per molecule values at the same surface pressure with a surface pressure deviation of $0.05 \mathrm{mN} / \mathrm{m}$. The isotherms were recorded from $\pi=0$ to $32 \mathrm{mN} / \mathrm{m}$ showing an increase of the lipid packing with increased PIP2 concentration.

Figure 4.23 shows averaged isotherms of POPC/POPE (80:20) and POPC/POPE/PIP2 (80-x:20:x, with $x=1,5,10 \mathrm{~mol} \%)$ measured in water (Figure $4.23 \mathrm{~A}$ ) and HEPES buffer (Figure $4.23 \mathrm{~B}$ ). In all cases, the surface pressure increased with reduced area 
per molecule $A_{\mathrm{m}}$. None of the isotherms showed a coexistence region (cf. Section 3.3.5). The isotherms were shifted to lower $A_{\mathrm{M}}$ values when the $\mathrm{PIP}_{2}$ concentration increased from 1 mol\% $\mathrm{PIP}_{2}$ to $10 \mathrm{~mol} \%$ PIP2. Thus, increasing the $\mathrm{PIP}_{2}$ content led to a higher packing density or to the loss of lipids to the subphase. Within the errors, no significant differences of the isotherms in buffer or water were observed. The isotherms of the lipid composition POPC/POPE (80:20) were similar to those of POPC/POPE/PIP 2 (75:20:5). Some monolayers with 1 mol\% $\mathrm{PIP}_{2}$ on HEPES buffer collapsed at high surface pressures (about 27-30 mN/m), indicated by the drop of $\pi$. In the averaged isotherm of this lipid composition it is represented by a sudden shift to smaller $A_{\mathrm{M}}$ above $25 \mathrm{mN} / \mathrm{m}$ (cf. Figure 4.23, light blue, Figure $4.23 \mathrm{~B}$ ). Thus, the maximum of the lipid packing was reached. To compare the isotherms, the $A_{20}$-values were listed in Table 4.8. The $A 20$ value describes the surface pressure at an area per molecule of $A_{\mathrm{M}}=20 \AA^{2}$ and allows to compare the lipid packing of different lipids at a certain surface pressure. At this certain surface pressure a collapse of all monolayer is excluded. The area per molecule values $(A \mathrm{M})$ were averaged over all lipids in the ratio of their molecular fraction.

Table 4.8: $A_{20}$ mean values with standard deviations obtained from the isotherms with the different lipid compositions. The subphases were ultrapure water and HEPES buffer (10 mM HEPES, $160 \mathrm{mM} \mathrm{KCl}, \mathrm{pH} 4$.7). $n$ describes the amount of recorded isotherms.

\begin{tabular}{|l|c|c|c|c|}
\hline & \multicolumn{2}{|c|}{ Subphase: ultrapure water } & \multicolumn{2}{c|}{ Subphase: HEPES buffer } \\
\hline Lipid composition & $A_{20} / \AA^{2}$ & $n$ & $A_{20} / \AA^{2}$ & $n$ \\
\hline POPC/POPE (80:20) & $64.6 \pm 2.0$ & 4 & $64.5 \pm 2.0$ & 4 \\
\hline $\begin{array}{l}\text { POPC/POPE/PIP2 } \\
\text { (79:20:1) }\end{array}$ & $68.6 \pm 0.8$ & 3 & $67.3 \pm 0.8$ & 3 \\
\hline $\begin{array}{l}\text { POPC/POPE/PIP2 } \\
\text { (75:20:5) }\end{array}$ & $63.1 \pm 2.4$ & 3 & $66.5 \pm 0.1$ & 3 \\
\hline $\begin{array}{l}\text { POPC/POPE/PIP2 } \\
\text { (70:20:10) }\end{array}$ & $57.8 \pm 1.7$ & 4 & $57.4 \pm 0.8$ & 3 \\
\hline
\end{tabular}

The $A_{20}$ values show that the buffer did not influence the lipid packing, but the $\mathrm{PIP}_{2}$ content did. With an increasing $\mathrm{PIP}_{2}$ concentration, the area per molecule slightly decreased indicating a denser lipid packing of the monolayers. Within the errors the 
POPC/POPE lipid mixture showed similar area per molecules values compared to the lipid compositions with $1 \mathrm{~mol} \%$ and $5 \mathrm{~mol} \%$.

In order to investigate if the monolayers composed of POPC/POPE and POPC/POPE/PIP 2 were stable over time, the change in surface pressure after reaching a value above $30 \mathrm{mN} / \mathrm{m}$ was recorded. In cell membranes, the lipid packing density has an area per molecule of 40-70 $\AA^{2}$, which is equivalent to a monolayer at this surface pressure. ${ }^{113,114}$ At the beginning, the monolayers were compressed to the desired surface pressure, then the barrier motor was stopped to keep the trough area constant and the surface pressure decay was observed. The monolayer with $1 \mathrm{~mol} \%$ did not collapse during these experiments. Figure 4.24 shows an exemplary plot of the surface pressure over $1 \mathrm{~h}$ for a POPC/POPE/PIP2 (75:20:5) monolayer.

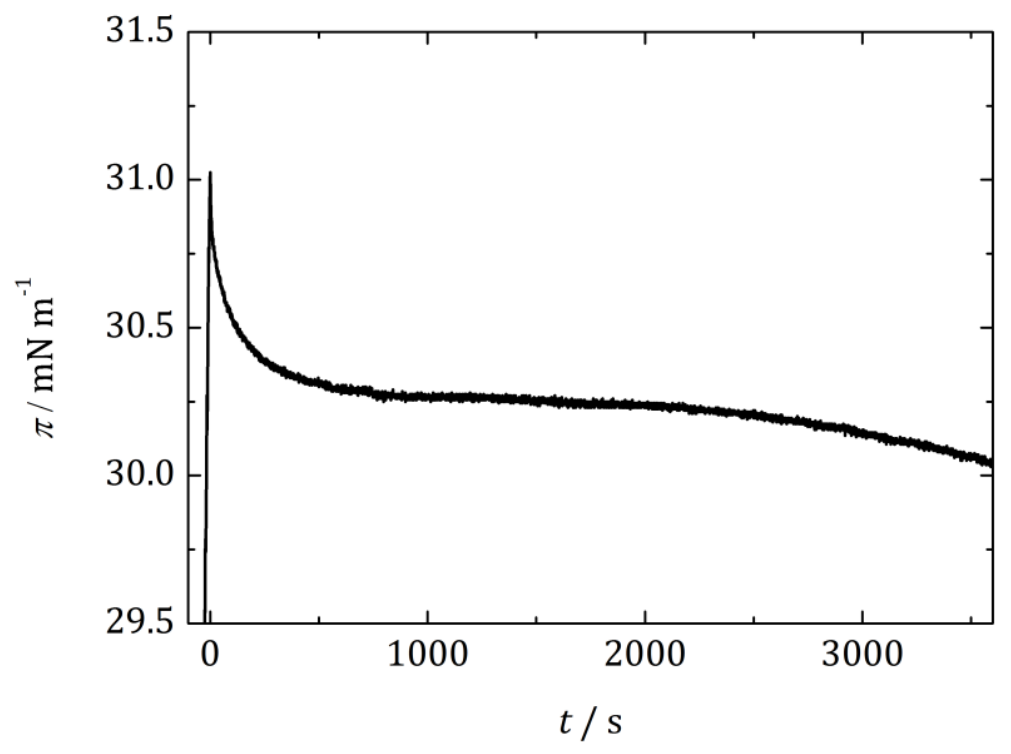

Figure 4.24: Exemplary plot of the surface pressure vs. time of a POPC/POPE/PIP 2 (75:20:5) monolayer. After reaching the desired surface pressure (above $30 \mathrm{mN} / \mathrm{m}$ ) the barriers were stopped at $t=0 \mathrm{~s}$, keeping the trough area constant. The change in surface pressure was detected over one hour to determine the change in surface pressure and to obtain information about the monolayer stability. The measurement was performed at $20^{\circ} \mathrm{C}$ with HEPES buffer as subphase.

After the trough area was held constant, the surface pressure decreased fast about $0.7 \mathrm{mN} / \mathrm{m}$, which was attributed to the barrier motor stop. Even though this behavior was observed often, the surface pressure stabilized after a time and the change in surface pressure got small. The total surface pressure loss was $2 \pm 3 \%$ for 
$1 \mathrm{~mol} \% \mathrm{PIP}_{2}(n=2), 4 \pm 1 \%$ for $5 \mathrm{~mol} \% \mathrm{PIP}_{2}(n=2)$ and $2 \pm 1 \%$ for $10 \mathrm{~mol} \% \mathrm{PIP}_{2}(n=3)$ over 1 h. The POPC/POPE monolayers without PIP 2 showed a surface pressure loss of $1 \pm 3 \%$. If the surface pressure decreased less than $1 \mathrm{mN} / \mathrm{m}$ per minute the monolayer was defined as stable. ${ }^{115}$ Although it could not be excluded that $\mathrm{PIP}_{2}$ or other lipids submerged into the subphase during the compression, once $30 \mathrm{mN} / \mathrm{m}$ was reached, the monolayers with the different lipid compositions were sufficiently stable to perform insertion experiments.

As monolayer stability was guaranteed, penetration experiments of ENTH into POPC/POPE and POPC/POPE/PIP2 monolayers were done at different initial surface pressures $\left(\pi_{0}\right)$ with a protein concentration of $1 \mu \mathrm{M} . \Delta \pi$ is dependent on the protein concentration and reaches a saturation at $c_{\mathrm{ENTH}}=0.2 \mu \mathrm{M} .{ }^{25}$ Therefore, at higher concentrations the maximal change in the surface pressure $(\Delta \pi)$ should not be influenced by the protein concentration and consequently represents the maximum value for $\Delta \pi$. The isotherms were performed on a Teflon trough, comprising a volume of $120 \mathrm{~mL}$. To reduce the amount of necessary protein to yield a concentration of $1 \mu \mathrm{M}$, the monolayer penetration experiments were performed with a custom-built protein trough $(V=2 \mathrm{~mL}$, cf. Section 3.3.5). After the desired surface pressure was reached, the small Teflon barriers were inserted into the protein trough. If the surface pressure remained stable, ENTH was added to the subphase via a syringe. To distribute the protein the system was then mixed with the syringe. Addition of the protein resulted in an increase of the surface pressure. Figure 4.25 represents an exemplary time-resolved increase of $\pi$ after injection of $1 \mu \mathrm{M}$ ENTH to a POPC/POPE/PIP2 (70:20:10) monolayer at an initial surface pressure of $14.6 \mathrm{mN} / \mathrm{m}$. 


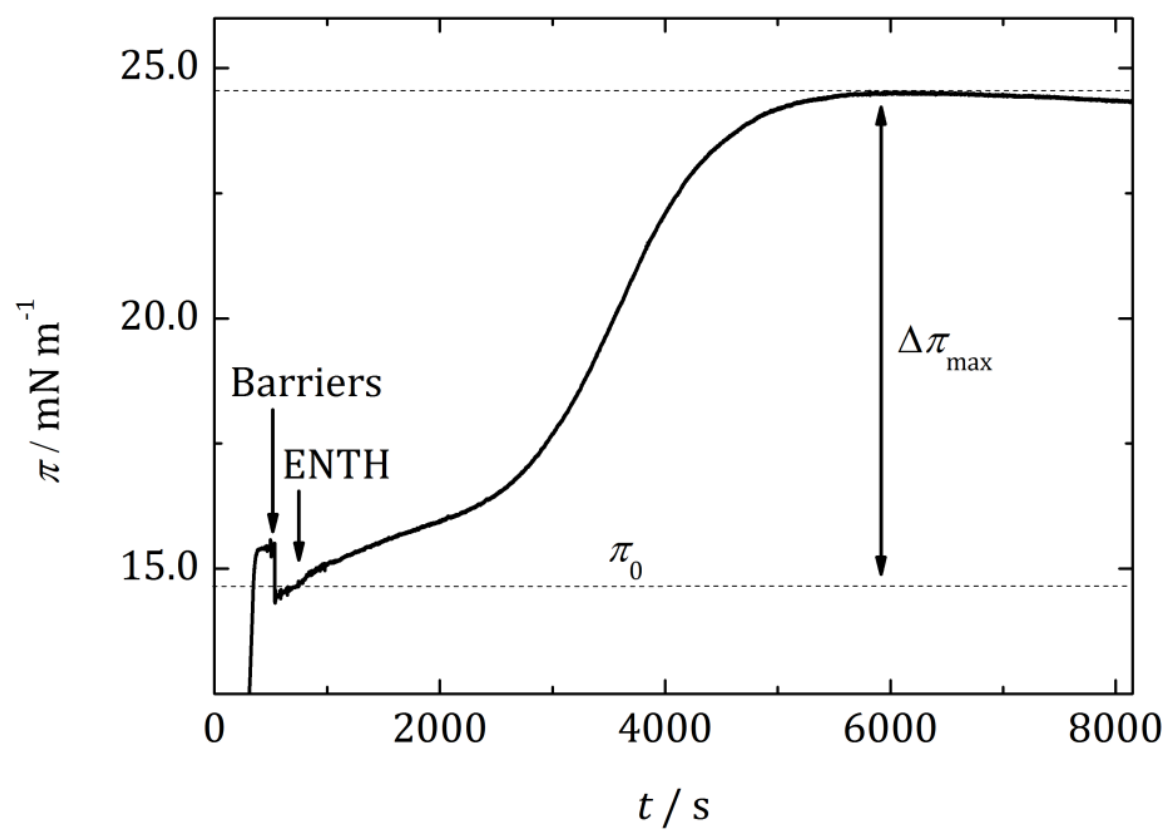

Figure 4.25: Exemplary plot of the surface pressure vs. time of a POPC/POPE/PIP 2 (70:20:10) monolayer after ENTH addition at $20^{\circ} \mathrm{C}$ using a protein trough. When the desired surface pressure was reached $(14.6 \mathrm{mN} / \mathrm{m})$ the small Teflon barriers were implemented in the protein trough, resulting in a drop in surface pressure. When no change in surface pressure was observed, the ENTH domain was added via a small hole in the trough. Due to protein-monolayer interactions and penetration ability of the ENTH domain, the surface pressure increased reaching a maximum after $6000 \mathrm{~s}$.

After the insert of the small protein trough barriers, a sudden decrease of $\pi$ of about $1 \mathrm{mN} / \mathrm{m}$ was observed. Once $\pi$ stabilized (usually within a few minutes), $1 \mu \mathrm{M}$ ENTH was injected into the subphase. Dependent on the initial surface pressure $\left(\pi_{0}\right), \pi$ reached a maximum after 10-210 min. In general the time to reach the maximum increased with decreased initial surface pressure as the surface pressure change $\Delta \pi$ was higher at those conditions due to a looser lipid packing of the monolayer. The increase of $\pi$ usually did not increased exponential, exhibiting local extrema. Directly after insertion of ENTH the $\pi$ increased fast until it almost reached a plateau (Figure 4.25) or even decreased. Then $\pi$ increased fast again. This behavior was observed several times and was attributed to the uneven distribution of the protein as the system was just mixed with a syringe directly after protein addition. Afterwards the ENTH was only distributed by diffusion.

To calculate the exclusion or critical surface pressure $\left(\pi_{c}\right)$ at which no further protein insertion was possible into POPC/POPE (80:20) and POPC/POPE/PIP2 (80-x:20:x, 
with $\mathrm{x}=1 ; 5$ and $10 \mathrm{~mol} \%$ ) monolayers, $\Delta \pi$ was plotted against $\pi_{0}$ (Figure 4.26). Interpolation yielded the intersection with the $\mathrm{x}$-axis, the critical surface pressure.

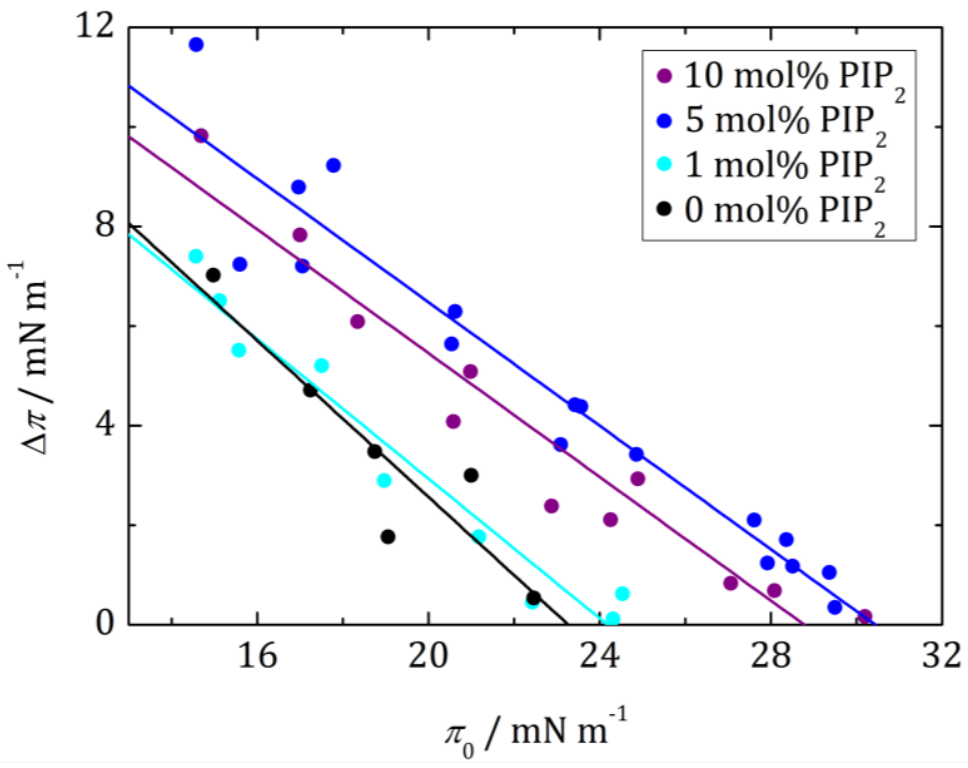

Figure 4.26: Monolayer penetration of the ENTH domain into POPC/POPE (80:20) and POPC/POPE/PIP2 (80-x:20:x, with x=1,5,10) monolayers. Maximum increase in surface pressure $(\Delta \pi)$ was monitored as a function of $\pi_{0}$ values. The subphase was $10 \mathrm{mM}$ HEPES, $160 \mathrm{mM} \mathrm{KCl}, \mathrm{pH}$ 7.4.

Figure 4.26 shows the $\Delta \pi$ at initial surface pressures of $\pi_{0}=14-30 \mathrm{mN} / \mathrm{m}$ for four different lipid mixtures. First of all, monolayers lacking PIP 2 were used to check the surface activity or the penetration ability of ENTH independently of the receptor binding. Towards this lipid mixture, the ENTH domain had a low intrinsic penetration capability with a $\pi_{\mathrm{c}}=23.2 \pm 0.8 \mathrm{mN} / \mathrm{m}$.

When the concentration of PIP 2 was increased to 1 mol\%, the $\pi_{c}$ just slightly increased to $24.1 \pm 0.5 \mathrm{mN} / \mathrm{m}$. At higher $\mathrm{PIP}_{2}$ content, the penetration ability of ENTH into the monolayers increased $\left(\pi_{\mathrm{c}}=30.3 \pm 0.4 \mathrm{mN} / \mathrm{m}\right.$ for $5 \mathrm{~mol} \%$ and $\pi_{\mathrm{c}}=28.7 \pm 0.6 \mathrm{mN} / \mathrm{m}$ for $10 \mathrm{~mol} \%$ ). The errors were given as weighted standard deviations of the fits. In Figure 4.27 the area per molecule at $20 \mathrm{mN} / \mathrm{m} \mathrm{(A)}$ and the critical surface pressure after ENTH addition (B) were plotted against the $\mathrm{PIP}_{2}$ concentration, showing the influence of the receptor lipid concentration as well as the lipid packing on the ENTH penetration ability. To ensure that the monolayer did not collapse at high pressures, the area per molecule at $20 \mathrm{mN} / \mathrm{m}$ was chosen to compare the lipid packing density. Considering the $\pi_{\mathrm{c}}$ value of the monolayers with $5 \mathrm{~mol} \%(30.3 \pm 0.4 \mathrm{mN} / \mathrm{m})$ to $10 \mathrm{~mol} \% \mathrm{PIP}_{2}(28.7 \pm 0.6 \mathrm{mN} / \mathrm{m})$ no significant differences were observed indicating 
that a maximum of the ENTH penetration was reached. Monolayers with $10 \mathrm{~mol} \%$ $\mathrm{PIP}_{2}$ showed a denser lipid packing compared to the monolayer with 5 mol\% $\mathrm{PIP}_{2}$.

A

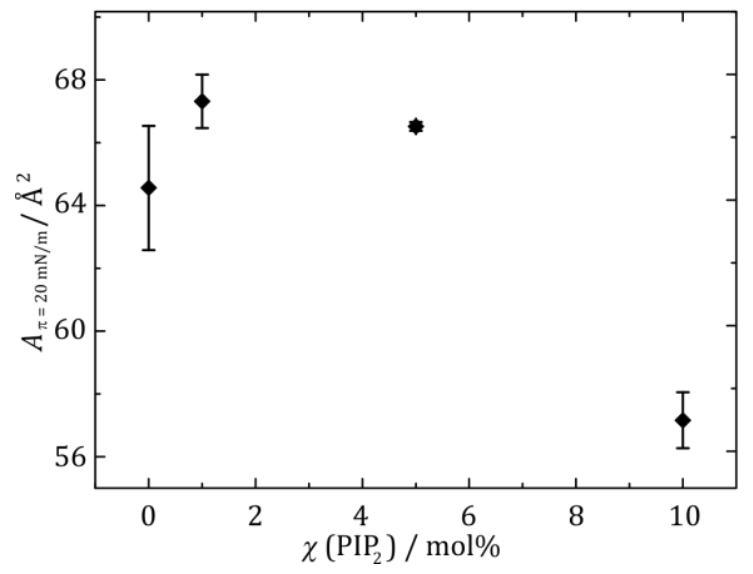

B

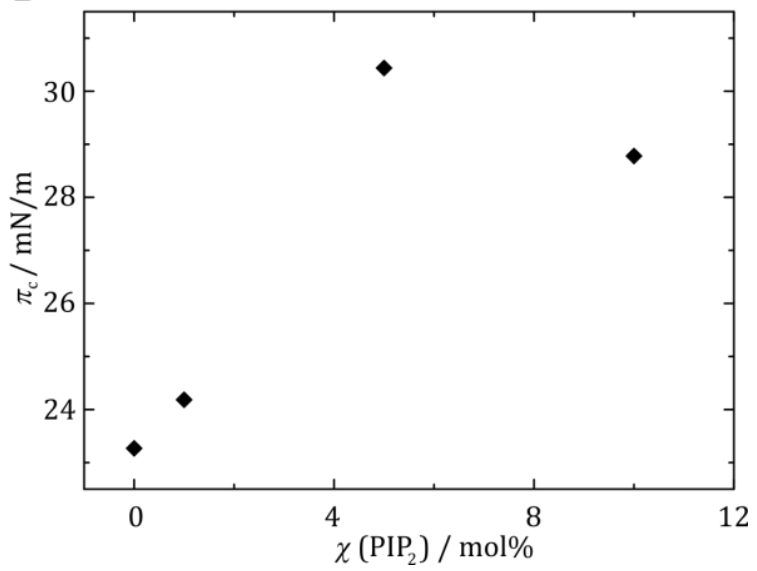

Figure 4.27: (A) Lipid packing against $\mathrm{PIP}_{2}$ concentration and (B) the critical surface pressure against the $\mathrm{PIP}_{2}$ content. The area per molecule at the beginning increases by addition of $\mathrm{PIP}_{2}$ and then decreases with increased $\mathrm{PIP}_{2}$ concentration. The critical surface pressure also first rises by the addition of $\mathrm{PIP}_{2}$, reaches a maximum at $5 \mathrm{~mol} \%$ and then decreases again indicating that at some point the lipid packing prevents further protein penetration.

In general, the $\Delta \pi$ values for lipid mixtures with $5 \mathrm{~mol} \% \mathrm{PIP}_{2}$ were about $1 \mathrm{mN} / \mathrm{m}$ higher as the data measured on $10 \mathrm{~mol} \% \mathrm{PIP}_{2}$ monolayers. Even though the scattering of the data points is quite high, this indicated a maximum of the penetration capability of ENTH into monolayers with 5 mol\% $\mathrm{PIP}_{2}$.

\subsubsection{PS influence on the ENTH penetration ability}

The influence of PS on the ENTH-PIP2 interaction was already described earlier. 8,26 Also RIfS measurements showed a higher binding affinity of ENTH to $\mathrm{PIP}_{2}$ containing supported bilayers in the presence of PS (cf. Section 4.2.2). To investigate the influence of PS on the penetration capability of ENTH to PIP $_{2}$-containing monolayers, first of all the intermolecular interactions within POPC/POPE (80:20), POPC/POPE/POPS (60:20:20) and POPC/POPE/POPS/PIP2 (55:20:20:5) monolayers were analyzed 
with surface pressure-area isotherms on ultrapure water and HEPES as subphases. Averaged isotherms of these lipid compositions are shown in Figure 4.28.

A

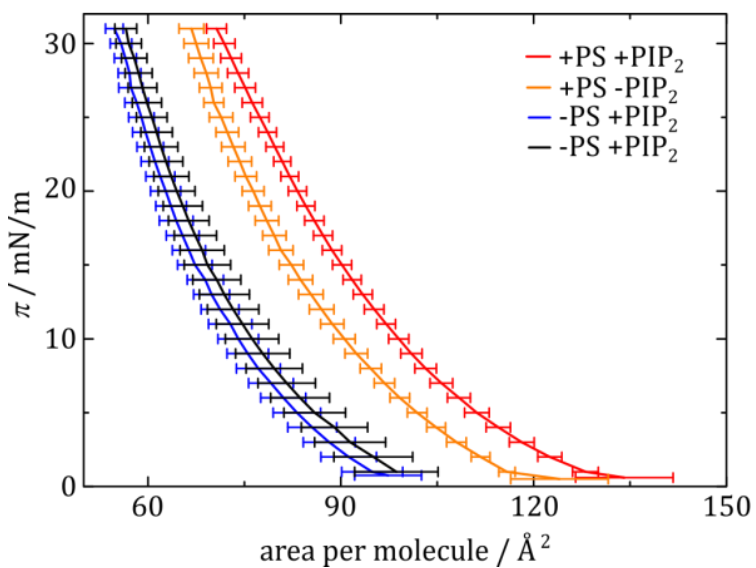

B subhase: HEPES buffer

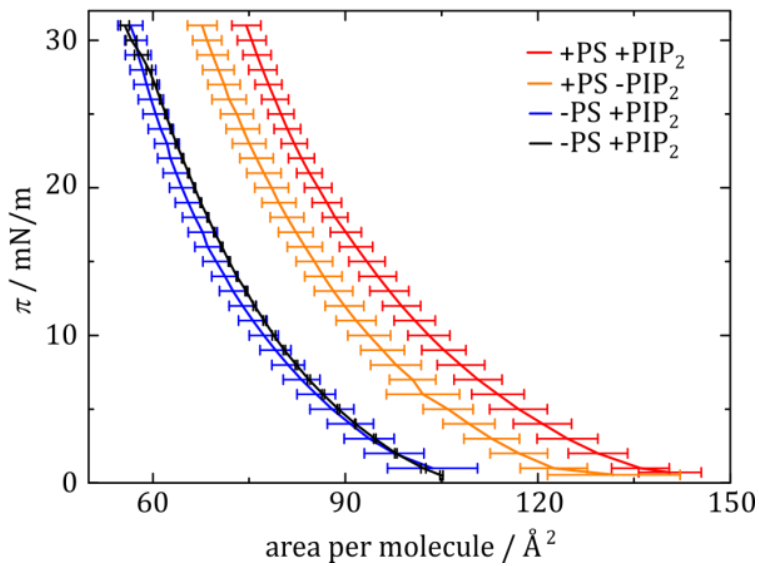

Figure 4.28: Averaged surface pressure-area per molecule isotherms of POPC/POPE (80:20, black, $n=4)$, POPC/POPE/PIP2 $(75: 20: 5$, blue, $n=3)$, POPC/POPE/POPS (60:20:20, orange, $n=3)$ and POPC/POPE/POPS/PIP $2(55: 20: 20: 5$, red, $n=3)$ at $20^{\circ} \mathrm{C}$. The subphases were ultrapure water $(\mathrm{A})$ and HEPES buffer (B). The error bars represent the standard deviations of the area per molecule at the same surface pressure with a surface pressure deviation of $0.05 \mathrm{mN} / \mathrm{m}$. The isotherms were recorded from $\pi=0$ to $31 \mathrm{mN} / \mathrm{m}$, showing the influence of the PS on the lipid packing of the monolayers as the isotherms are shifted to higher molecule per area values. No significant difference between the isotherms on water or HEPES buffer were observed.

Independent of the lipid mixture the curve shape of all isotherms resemble one another. When decreasing the trough area, the surface pressure steadily increased and no co-existence region was observed (cf. Section 3.3.5). It was possible to compress the POPC/POPE and POPC/POPE/PIP2 monolayers further than the monolayers containing PS. The isotherm of the POPC/POPE/POPS/PIP 2 monolayer showed the highest area per molecule values of the lipids for the same surface pressure compared to the other mixtures, indicating that POPS decreased the lipid packing of the monolayers. Especially in combination with $\mathrm{PIP}_{2}$ the lipid packing in the monolayers was decreased. Within the error margins the isotherms on HEPES buffer did not differ from the isotherms on water. Furthermore the lipid mixtures were stable up to the $\pi$ values of $31 \mathrm{mN} / \mathrm{m}$, allowing the measurement of the ENTH penetration capability. As al- 
ready mentioned in chapter 4.3.1, the $A 20$ values were determined to obtain information about the lipid packing (Table 4.9) and to compare them. The area per molecule values $(A \mathrm{M})$ were averaged over all lipids in the ratio of their molecular fraction.

Table 4.9: $A_{20}$ mean values with standard deviations obtained from the isotherms with the different lipid compositions. The subphases were ultrapure water and HEPES buffer. $n$ is the number of recorded isotherms.

\begin{tabular}{|c|c|c|c|c|}
\hline & \multicolumn{2}{|c|}{ Subphase: ultrapure water } & \multicolumn{2}{c|}{ Subphase: HEPES buffer } \\
\hline Lipid composition & $A_{20} / \AA^{2}$ & $n$ & $A_{20} / \AA^{2}$ & $n$ \\
\hline POPC/POPE (80:20) & $64.6 \pm 2.0$ & 4 & $64.5 \pm 2.0$ & 4 \\
\hline $\begin{array}{c}\text { POPC/POPE/POPS } \\
(60: 20: 20)\end{array}$ & $76.3 \pm 2.0$ & 3 & $79.9 \pm 4.0$ & 5 \\
\hline $\begin{array}{c}\text { POPC/POPE/PIP2 } \\
(55: 20: 5)\end{array}$ & $63.1 \pm 2.4$ & 3 & $64.6 \pm 0.1$ & 3 \\
\hline $\begin{array}{c}\text { POPC/POPE/POPS/PIP2 } \\
\text { (55:20:20:5) }\end{array}$ & $83.3 \pm 1.4$ & 3 & $83.9 \pm 4.2$ & 6 \\
\hline
\end{tabular}

The $A_{20}$ values show the influence of PS on the lipid packing within the monolayers. Comparing POPC/POPE (80:20) and POPC/POPE/PIP2 (75:20:5) monolayers the lipid packing got looser when PS was added, indicated by the increase of the $A_{20}$ value. Monolayers containing PS and PIP2 even showed higher $A_{20}$ values and consequently had a lower packing density.

Although, no collapse of the monolayers was observed until $31 \mathrm{mN} / \mathrm{m}$, it was necessary to investigate the monolayer stability over time to ensure the accessibility of the receptor lipid during the experiment (cf. Section 3.3.5). Therefore, the surface pressure change $(\Delta \pi)$ was recorded over time to obtain the percentage loss in surface pressure after $1 \mathrm{~h}$ for POPC/POPE/POPS (60:20:20) and POPC/POPE/POPS/PIP2 (55:20:20:5) monolayers. Over $1 \mathrm{~h}$ the surface pressure increased about $1 \pm 1 \%$ for the monolayers without $\mathrm{PIP}_{2}$ and decreased about $6 \pm 2 \%$ when $\mathrm{PIP}_{2}$ was present, indicating sufficient monolayer stability. ${ }^{115}$ 
With these stable monolayers penetration experiments of ENTH were possible. The experimental conditions were similar to the experiments shown in chapter 4.3.1. After the adjusted surface pressure was reached, the small barriers were implemented. When almost no change in $\pi$ was observed, indicating stable conditions, $1 \mu \mathrm{M}$ ENTH was added into the subphase and then mixed with the syringe. To investigate how the lipid PS influences the penetration ability of ENTH, the increases of $\Delta \pi$ for POPC/POPE/POPS (60:20:20) and POPC/POPE/POPS/PIP 2 (55:20:20:5) monolayers dependent on the initial surface pressure $\left(\pi_{0}\right)$ were analyzed. At low $\pi_{0}$ the change in surface pressure was greater compared to higher $\pi_{0}$ values due to the lower packing of the monolayer. When PS and $\mathrm{PIP}_{2}$ were present the maximum of $\pi$ was reached after 60-270 $\mathrm{min}$, whereby the time usually increased with decreasing $\pi_{0}$. Some irregularities in the surface pressure increase were attributed to inhomogeneous ENTH distribution (cf. chapter 4.3.1)

To obtain the penetration capability of ENTH into POPC/POPE/POPS (60:20:20) and POPC/POPE/POPS/PIP2 (55:20:20:5) monolayers, the data was plotted in a $\Delta \pi-\pi 0$ diagram. Furthermore the data for the POPC/POPE/PIP2 (cf. section 4.3.1) lipid mixture was added. Consequently, this allowed the comparision of all lipid mixtures and the influence of PS on the penetration ability. By interpolating $\pi_{\mathrm{c}}$ was determined (Figure 4.29). 


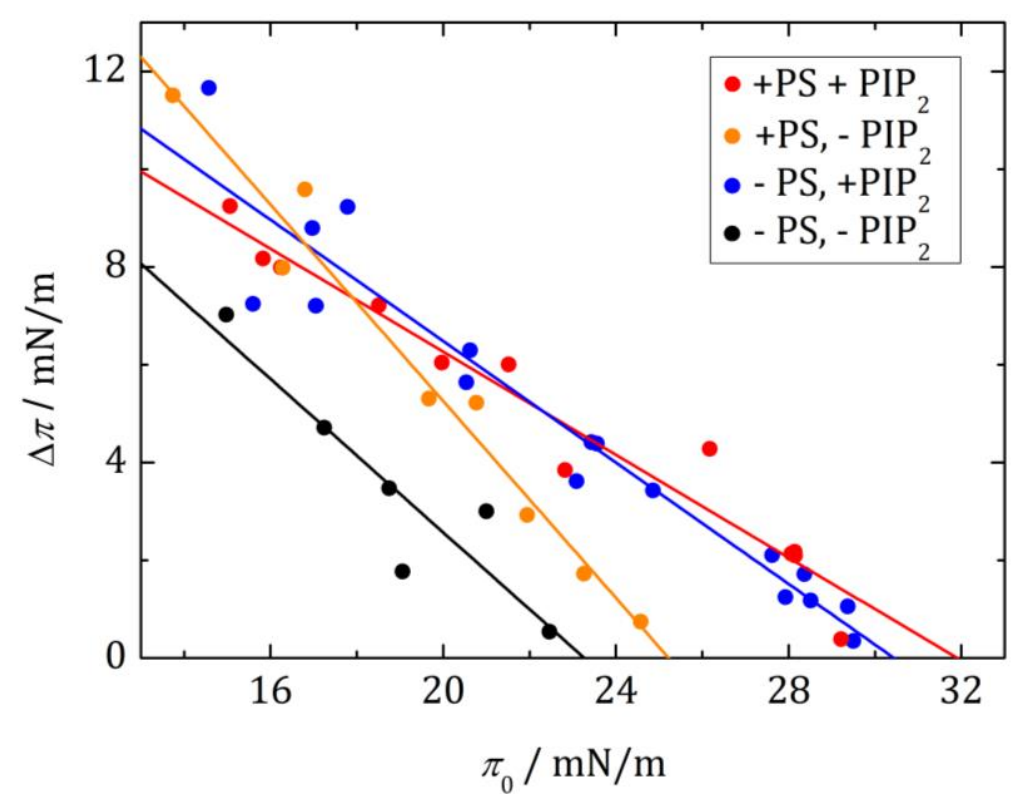

Figure 4.29: Monolayer penetration of the ENTH domain into POPC/POPE (80:20,), POPC/POPE/POPS (60:20:20), POPC/POPE/PIP2 (75:20:5) and POPC/POPE/POPS/PIP2 (55:20:20:5) monolayers. Maximum increase in surface pressure $(\Delta \pi)$ was monitored as a function of $\pi_{0}$ values. The subphase was $10 \mathrm{mM}$ HEPES, $160 \mathrm{mM} \mathrm{KCl}$, pH 7.4.

The $\mathrm{x}$-intercepts in Figure 4.29 of the fits reveal a mean $\Delta \pi_{\mathrm{c}}$ of $30.3 \pm 0.4 \mathrm{mN} / \mathrm{m}$ for POPC/POPE/PIP2 monolayers. Penetration experiments of ENTH into POPC/POPE/POPS/PIP2 monolayers yielded a $\pi_{c}$ of $32.0 \pm 0.9 \mathrm{mN} / \mathrm{m}$. The fit with PS shows a slighter decrease of $\Delta \pi$ with increasing $\pi_{0}$. Within the scatter of the measurements the values are similar. The experiments without PIP2 showed that the ENTH penetration ability decreased down to $23.2 \pm 0.8 \mathrm{mN} / \mathrm{m}$ for POPC/POPE monolayers and $25.0 \pm 1.0 \mathrm{mN} / \mathrm{m}$ for the POPC/POPE/POPS monolayers. The errors were given as weighted standard deviations of the fits. This reveals that the penetration capability of the ENTH domain is clearly decreased when $\mathrm{PIP}_{2}$ is lacking even though PS is present. Furthermore, the penetration ability of ENTH into monolayers composed of POPC/POPE/POPS/PIP2 only slightly increased compared to POPC/POPE/PIP2 monolayers, indicating that PS has almost no impact on the penetration capability of ENTH. 


\subsection{Adhered GUVs as a membrane model to investigate protein- membrane interactions}

The surface activity and especially the insertion of the amphipathic helix of ENTH resulted in an increase of the surface pressure in monolayer penetration experiments (cf. chapters 4.3.1 and 4.3.2). It was shown that the lipid composition has an influence on the exclusion pressure, although the effect of PS was only slight. Another possibility to investigate the activity of ENTH is the analysis of the activity as a function of membrane tension in bilayers. ${ }^{36}$

In this work, GUVs were adhered to a biotin-PEG functionalized glass surface to immobilize them. By using fluorescence microscopy the vesicle geometry was determined, which could be evaluated to get the membrane tension. Afterwards, ENTH was added to see how the protein effects the membrane tension. Based on this the influence of the lipid head group Phosphatidylserine (PS) on the binding behavior of ENTH as a function of lateral membrane tension was investigated.

\subsubsection{Theoretical model}

Adhesion of vesicles or cells were already used to investigate membrane-protein interactions. ${ }^{116}$ In this thesis, the membrane tension of adhered GUVs, generated as described in chapter 3.2.2, was analyzed to determine the impact of ENTH on the lateral membrane tension as a function of the lipid composition.

Attractive forces between the GUV and the surface result in the adhesion of the GUV. During this process the volume of the GUV remains constant under iso-osmolar conditions due to low water permeability of membranes. In contrast to this, the vesicle shape changes from a sphere with a radius $r_{\mathrm{v}}$ to a spherical cap with a radius $r_{\mathrm{ad}}$. Additionally, an adhesion area or contact area with a radius $r_{\mathrm{i}}$ is observed (Figure 4.30). From the geometry of the adhered vesicles it is then possible to calculate the membrane tension. 
A

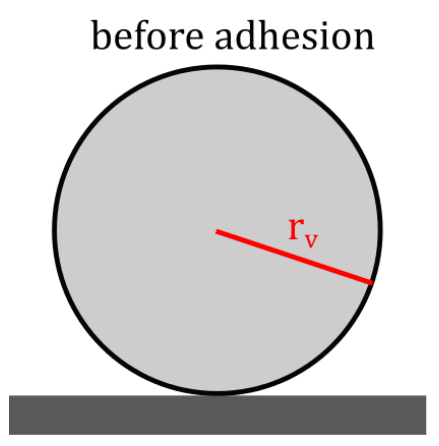

$\mathrm{B}$ after adhesion

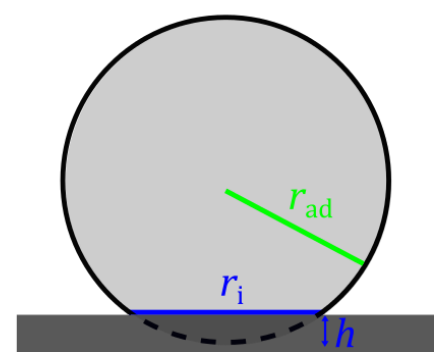

Figure 4.30: Schematic illustration of a GUV before (A) and after (B) adhesion to a surface. The GUV geometry changes from a sphere with a radius $r_{\mathrm{v}}$ to a spherical cap with a radius $r_{\mathrm{ad}}$ and a contact area (radius $r_{i}$ ). $h$ describes the height of the spherical cap in (B), which is missing to get a complete sphere.

Marsh theoretically described the observable tension of a fluctuating membrane using equation (4.5): ${ }^{117}$

$$
\sigma=\Delta \sigma-\frac{K_{\mathrm{A}} k_{B} T}{2 A_{\mathrm{V}} \kappa} \sum_{q}\left(\frac{\sigma}{\sigma q^{2}+\kappa q^{4}}\right)
$$

$K_{\mathrm{A}}$ is the area compressibility modulus, $k_{\mathrm{B}}$ the Boltzmann constant, $T$ the absolute temperature, $\kappa$ the bending rigidity and $A_{\mathrm{v}}$ the area of a free vesicle. $\Delta \sigma$ depicts the increase in membrane tension of a tension free planar membrane and is proportional to the fractional area expansion $\left(\Delta A / A_{\mathrm{v}}\right)$ and $K_{\mathrm{A}}$ (equation (4.5)).

$$
\Delta \sigma=K_{\mathrm{A}} \frac{\Delta A}{A_{\mathrm{v}}}
$$

Integration with $q_{\min }=\pi / \sqrt{A_{\mathrm{M}}}$ and $q_{\max }=\pi / \sqrt{A_{\mathrm{V}}}$, where $A_{\mathrm{M}}$ is the area per lipid, the observable tension $\sigma$ of a fluctuating membrane is then calculated with equation (4.7).

$$
\sigma=\Delta \sigma-\frac{K_{\mathrm{A}} k_{B} T}{8 \pi \kappa} \ln \left(\frac{\frac{\sigma A_{\mathrm{v}}}{\pi^{2} \kappa}+1}{\frac{\sigma A_{\mathrm{M}}}{\pi^{2} \kappa}+1}\right)
$$

The denominator of the logarithm becomes close to unity when the membrane tension is low $\left(\sigma \ll \pi^{2} \kappa / A_{\mathrm{M}} \approx 2 \cdot 10^{3} \mathrm{mN} / \mathrm{m}\right)$, simplifying equation (4.7): 


$$
\sigma=\Delta \sigma-\frac{K_{\mathrm{A}} k_{B} T}{8 \pi \kappa} \ln \left(\frac{\sigma A_{\mathrm{v}}}{\pi^{2} \kappa}+1\right)
$$

Under the assumption of $K_{\mathrm{A}} \gg \sigma \gg \pi^{2} \kappa / A_{\mathrm{v}} \approx 10^{-6} \mathrm{mN} / \mathrm{m}$, which is valid for relevant membrane tensions, the membrane tension is described with equation (4.9) as $\left(\sigma A_{\mathrm{v}}\right) /\left(\pi^{2} \kappa\right)$ is $\gg 1$.

$$
\sigma=\Delta \sigma-\frac{K_{\mathrm{A}} k_{B} T}{8 \pi \kappa} \ln \left(\frac{\sigma A_{\mathrm{v}}}{\pi^{2} \kappa}\right)
$$

When equation (4.6) and (4.9) are combined, the membrane tension of an adhered vesicle can be calculated using equation (4.10)

$$
\sigma=K_{\mathrm{A}} \frac{\Delta A}{A_{\mathrm{v}}}-\frac{K_{\mathrm{A}} k_{B} T}{8 \pi \kappa} \ln \left(\frac{\sigma A_{\mathrm{v}}}{\pi^{2} \kappa}\right)
$$

The membrane tension of the adhered GUV minus the tension of the free vesicle $\left(\sigma_{0}\right)$ enables to compare the obtained membrane tension with different reference states from previous studies. ${ }^{118,119}$ Thereby, the flattening of fluctuations and the dilation of the area per molecule has to be considered.

$$
\frac{\Delta A}{A_{\mathrm{v}}}=\frac{\sigma-\sigma_{0}}{K_{\mathrm{A}}}-\frac{k_{B} T}{8 \pi \kappa} \ln \left(\frac{\sigma}{\sigma_{0}}\right)
$$

The fractional area expansion term can be calculated from the geometries of the GUVs before and after adhesion (Figure 4.30). The fractional area expansion is calculated with equation (4.12).

$$
\frac{\Delta A}{A_{\mathrm{v}}}=\frac{\mathrm{A}_{\mathrm{ad}}-A_{v}}{A_{\mathrm{v}}}=\frac{4 \pi r_{\mathrm{ad}}^{2}-2 \pi r_{\mathrm{ad}} h+\pi r_{\mathrm{i}}^{2}-4 \pi r_{\mathrm{v}}^{2}}{4 \pi r_{\mathrm{v}}^{2}}
$$

$h$ is the height of the spherical cap (Figure 4.30). Considering a constant volume of the vesicle during the adhesion process $\left(V_{\mathrm{v}}=V_{\mathrm{ad}}\right)$ results in equation (4.15).

$$
V_{V}=\frac{4}{3} \pi r_{\mathrm{v}}^{3}
$$




$$
\begin{gathered}
V_{\mathrm{ad}}=\frac{4}{3} \pi r_{\mathrm{ad}}^{3}-\frac{\pi h}{6}\left(3 r_{\mathrm{i}}^{2}+h^{2}\right) \\
\frac{4}{3} \pi r_{\mathrm{v}}^{3}=\frac{4}{3} \pi r_{\mathrm{ad}}^{3}-\frac{\pi h}{6}\left(3 r_{\mathrm{i}}^{2}+h^{2}\right)
\end{gathered}
$$

Determination of the radius of the immobile, free vesicles $r_{\mathrm{v}}$ and the height of the spherical cap $h$ is experimentally complicated to realize and can be described as a function of $r_{\mathrm{i}}$ and $r_{\mathrm{ad}}$ :

$$
\begin{gathered}
h=r_{\mathrm{ad}}-\sqrt{r_{\mathrm{ad}}^{2}-r_{\mathrm{i}}^{2}} \\
r_{\mathrm{v}}=\sqrt[3]{r_{\mathrm{ad}}^{3}-\frac{r_{\mathrm{ad}}-\sqrt{r_{a d}^{2}-r_{i}^{2}}}{8}\left(3 r_{\mathrm{i}}^{2}+\left(r_{\mathrm{ad}}-\sqrt{r_{\mathrm{ad}}^{2}-r_{\mathrm{i}}^{2}}\right)^{2}\right)}
\end{gathered}
$$

Normalization of the radii to $r_{\mathrm{ad}}$ gives dimensionless variables and substitution of them in equation (4.12) results in: ${ }^{79}$

$$
\frac{\Delta A}{A_{\mathrm{v}}}=\frac{4 \pi-2 \pi\left(1-\sqrt{1-\left(\frac{r_{\mathrm{i}}}{\mathrm{r}_{\mathrm{ad}}}\right)^{2}}+\pi\left(\frac{r_{\mathrm{i}}}{r_{\mathrm{ad}}}\right)^{2}\right.}{4 \pi\left(\sqrt[3]{1-\frac{2-\left(\frac{r_{\mathrm{i}}}{r_{\mathrm{ad}}}\right)^{2} \sqrt{1-\left(\frac{r_{\mathrm{i}}}{r_{\mathrm{ad}}}\right)^{2}}-2 \sqrt{1-\left(\frac{r_{\mathrm{i}}}{r_{\mathrm{ad}}}\right)^{2}}}{4}}\right)^{2}}-1
$$

Finally, the lateral membrane tension of the adhered GUV can be calculated numerically by the combination of equation (4.11) and (4.18). 


$$
\frac{\sigma-\sigma_{0}}{K_{\mathrm{A}}}+\frac{k_{B} T}{8 \pi \kappa} \ln \left(\frac{\sigma}{\sigma_{0}}\right)=\frac{4 \pi-2 \pi(1-b)+\pi\left(\frac{r_{\mathrm{i}}}{r_{\mathrm{ad}}}\right)^{2}}{4 \pi\left(\sqrt[3]{1-\frac{2-\left(\frac{r_{\mathrm{i}}}{r_{\mathrm{ad}}}\right)^{2} b-2 b}{4}}\right)^{2}}-1
$$

with $b=\sqrt{1-\left(\frac{r_{\mathrm{i}}}{r_{\mathrm{ad}}}\right)^{2}}$.

Rawicz et al. measured the area compressibility modulus and the bending rigidity for a DOPC membrane via micropipette aspiration and yielded a $K_{\mathrm{A}}$ of $265 \pm 18 \mathrm{mN} / \mathrm{m}$ and a $\kappa$ of $21 k_{\mathrm{B}} T\left((0.85 \pm 0.1) \cdot 10^{-19} \mathrm{~J}\right) \cdot{ }^{120}$ For GUVs weakly adhered to a solid support, pre-tensions $\left(\sigma_{0}\right)$ were measured to be in a range of $10^{-1}-10^{-3} \mathrm{mN} / \mathrm{m} .{ }^{121}$ Using the different pre-tension regimes, the lateral tension as a function of $r_{\mathrm{i}} / r_{\mathrm{ad}}$ first increases slow and then fast when the dilation exceeds the excess area, which is stored in membrane undulations. ${ }^{36,119}$

Calculation of the membrane tension of the adhered GUVs before and after ENTH addition hence can yield the protein activity as a function of membrane tension dependent of the lipid composition.

\subsubsection{Adhesion of biotinylated GUVs on PEGylated surfaces}

Adhered GUVs were already used to study membrane-protein interaction or phase separation of lipid bilayers. ${ }^{122-124}$ Gleisner et al. used Avidin coated glass surfaces to immobilize biotinylated GUVs and therewith analyzed the ENTH-PIP2 interactions as a function of membrane tension. ${ }^{36}$ They performed these experiments with adhered GUVs composed of DOPC/DOPE/cap-biotin-DOPE/Atto488-DPPE (67:30:2:1). The membrane tension of these GUVs were regulated by the magnesium chloride $\left(\mathrm{MgCl}_{2}\right)$ concentration in the buffer.

In this work, a similar system was used to investigate how the lipid head group PS influences the ENTH binding considering the membrane tension of the GUVs before and after ENTH addition. As PS and $\mathrm{PIP}_{2}$ exhibit negative charges and are known to 
cluster in the presents of divalent ions, ${ }^{125,126}$ the buffer and the functionalization of the surface were changed. $\mathrm{MgCl}_{2}$ was removed from the buffer and NeutrAvidin was used instead of avidin to immobilize the vesicles. ${ }^{36}$ NeutrAvidin is known to reduce the unspecific interactions compared to avidin. ${ }^{127}$

Direct functionalization of the surface with NeutrAvidin by simple incubation on a hydrophilized glass substrate led to an inhomogenous occupancy of the protein on the glass slides (Figure 4.31 A), which can be explained by less unspecific interactions of NeutrAvidin with the hydrophilic glass surface. As a homogenous coverage with the protein was necessary to obtain adhesion of biotinylated GUVs, the glass slide surfaces were silanized and PEGylated (PEG= Poly(ethylene glycol)) (cf. 3.2.2). Using biotin-linked PEG (b-PEG) ensured an affinity of NeutrAvidin to the surface and due to four biotin binding sides, additionally binding to the biotinylated vesicles was guaranteed. To ensure the accessibility of the biotin groups on the surface, methoxy functionalized PEGs (m-PEG) were added (ratio m-PEG/b-PEG 1:2). Similar PEGfunctionalizations were already used in previous studies. ${ }^{128,129}$ With the new functionalization method incubation with DyLight ${ }^{\circledR} 594$ labeled Neutravidin resulted in a homogenous NeutrAvidin coverage (Figure 4.31 B).
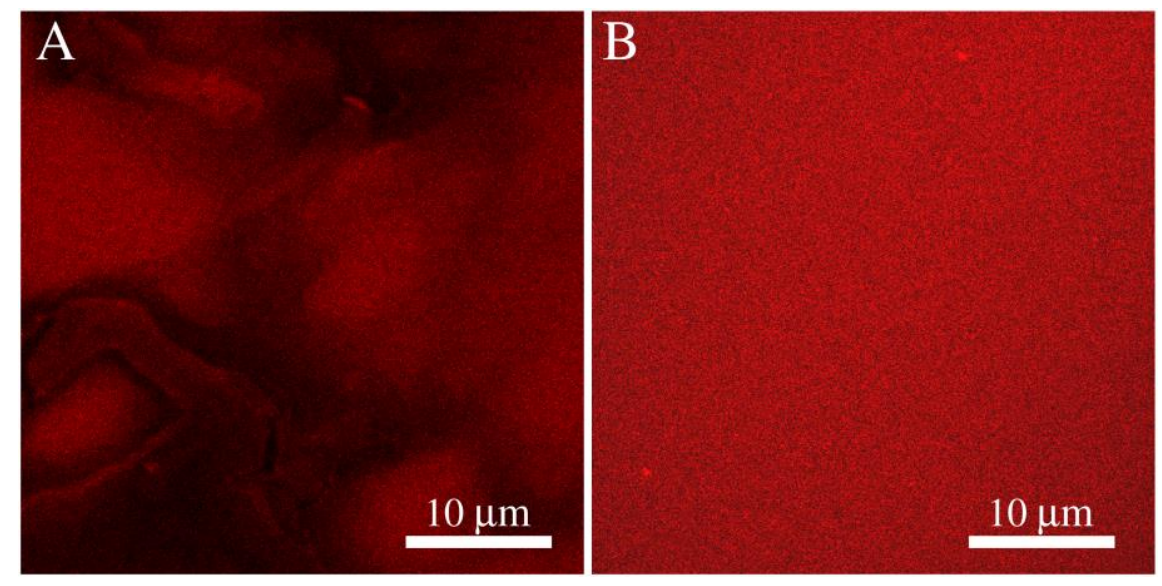

Figure 4.31: Fluorescence images of (A) hydrophilized and (B) biotin-PEG-functionalized glass slides after incubation with DyLight ${ }^{\circledR} 594$ labeled Neutravidin and subsequent rinsing with buffer. Without PEG-functionalization the protein was removed from the surface by rinsing with buffer resulting in an inhomogeneous coverage of NeutrAvidin. Due to the strong biotin-NeutrAvidin interaction the biotin an even distribution of the labeled protein on the PEG-functionalized surface was observed. 
Figure 4.31 clearly shows the specific NeutrAvidin-biotin interaction. In Figure $4.31 \mathrm{~A}$ the hydrophilized glass slides were just directly incubated with the protein. After rinsing with buffer NeutrAvidin was partly removed. When the glass slides were first silanized with (3-Glycidyloxypropyl)trimethoxysilane (GOPTS) and then PEGylated with a mixture of m-PEG and b-PEG (cf. chapter 3.2.2), the NeutrAvidin strongly bound to the surface, which was not removed by rinsing with buffer. After the successful coverage of the surface with NeutrAvidin biotinylated GUVs could be added to the substrates. GUVs composed of DOPC/DOPS/Cholesterol/cap-biotinDOPE/PIP2/Atto488-DPPE (76:10:10:2:1:1) turned out to be large and stable enough to perform these experiments. Higher concentrations of DOPS or DOPE in combination with the b-PEG-NeutrAvidin surface resulted in instable GUVs, preventing the analysis of adhesion areas and consequently the membrane tension.

Incubation of these GUVs on the PEG-surface for 15 min obtained immobilized GUVs with small adhesion areas (Figure 4.32). Fluorescence images of the adhered GUVs were taken by means of Spinning Disc confocal laser microscopy (SDCLM). To obtain the radii of the adhered GUV and the contact area (cf. 3.2.2), z-stacks of the GUVs with 150-250 nm between the slides were recorded.
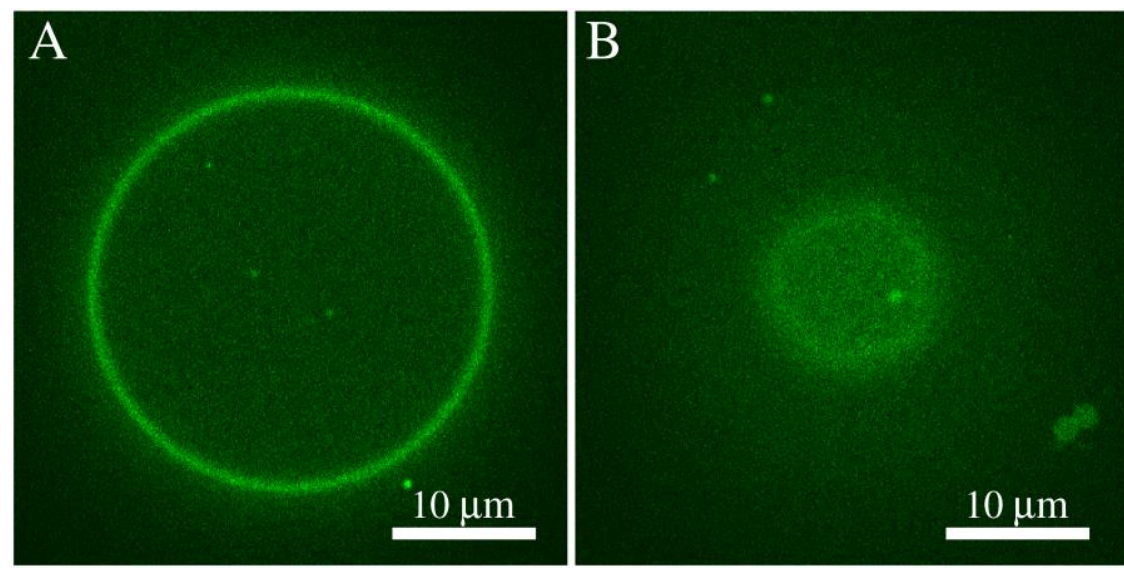

Figure 4.32: z-stacks of an adhered GUVs obtained by SDCLM. (A) Cross section of the adhered GUV with the radius $r_{\text {ad. }}$ (B) Fluorescence image of the contact area plane to determine $r_{\mathrm{i}}$. Lipid composition: DOPC/DOPS/Cholesterol/cap-biotin-DOPE/PIP2/Atto488-DPPE (76:10:10:2:1:1).

Calculation of the lateral tension from the geometry of the vesicles is only possible if the ratio $r_{\mathrm{i}} / r_{\mathrm{ad}}$ is constant over the experimental time. The radii were determined as 
described in chapter 3.2.2. Also after $2 \mathrm{~h}$ the adhered vesicles showed sufficient stability as the ratio $r_{\mathrm{i}} / r_{\mathrm{ad}}$ did not change within the errors (Figure 4.33).

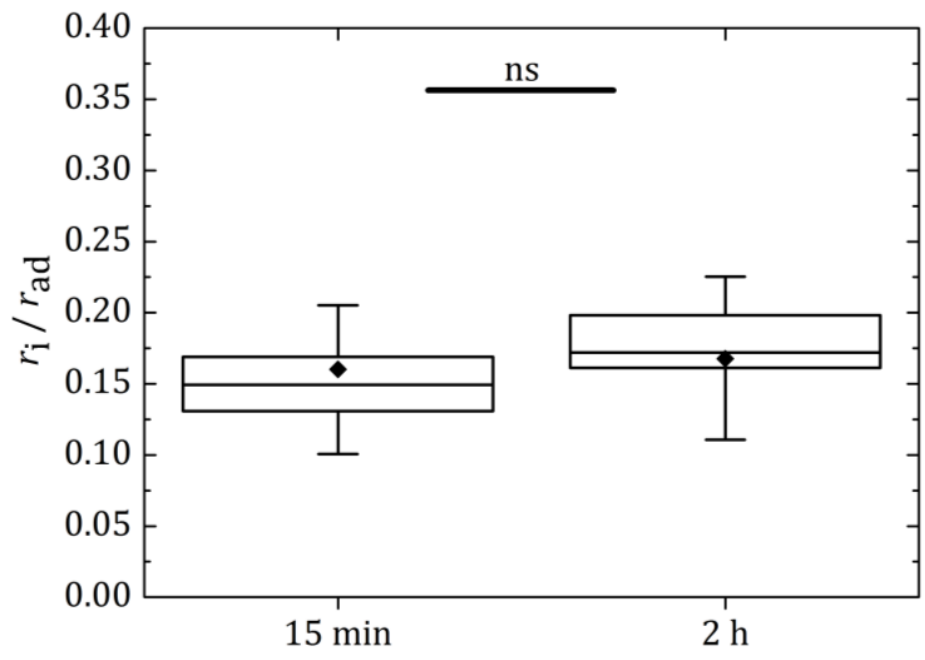

Figure 4.33: Ratios of the contact area radius to the adhered vesicle radius $\left(r_{\mathrm{i}} / r_{\mathrm{ad}}\right)$ as a function of time for GUVs (DOPC/DOPS/Cholesterol/cap-biotin-DOPE/PIP2/Atto488-DPPE (76:10:10:2:1:1)) adhered to a NeutrAvidin covered surface, indicating the sufficient stability of the GUVs after $2 \mathrm{~h}$. Statistical Mann-Whitney $U$ test: ns - not significant ( $p=0.3)$.

25 GUVs were analyzed after subsidence (15 min) and 16 GUVs after $2 \mathrm{~h}$, yielding $r_{\mathrm{i}} / r_{\mathrm{ad}}$ values of $0.16 \pm 0.04$ and $0.17 \pm 0.04$, respectively. The lateral membrane tension of the adhered GUVs was then calculated from the ratio of $r_{\mathrm{i}} / r_{\mathrm{ad}}$ using equation (4.19) with an area compressibility modulus of $K_{\mathrm{A}}=265 \mathrm{mN} / \mathrm{m}$, a bending rigidity of $\kappa=21 \mathrm{kBT}$ and a pre-tension of $\sigma_{0}=10^{-2} \mathrm{mN} / \mathrm{m}$ (Figure 4.34). 


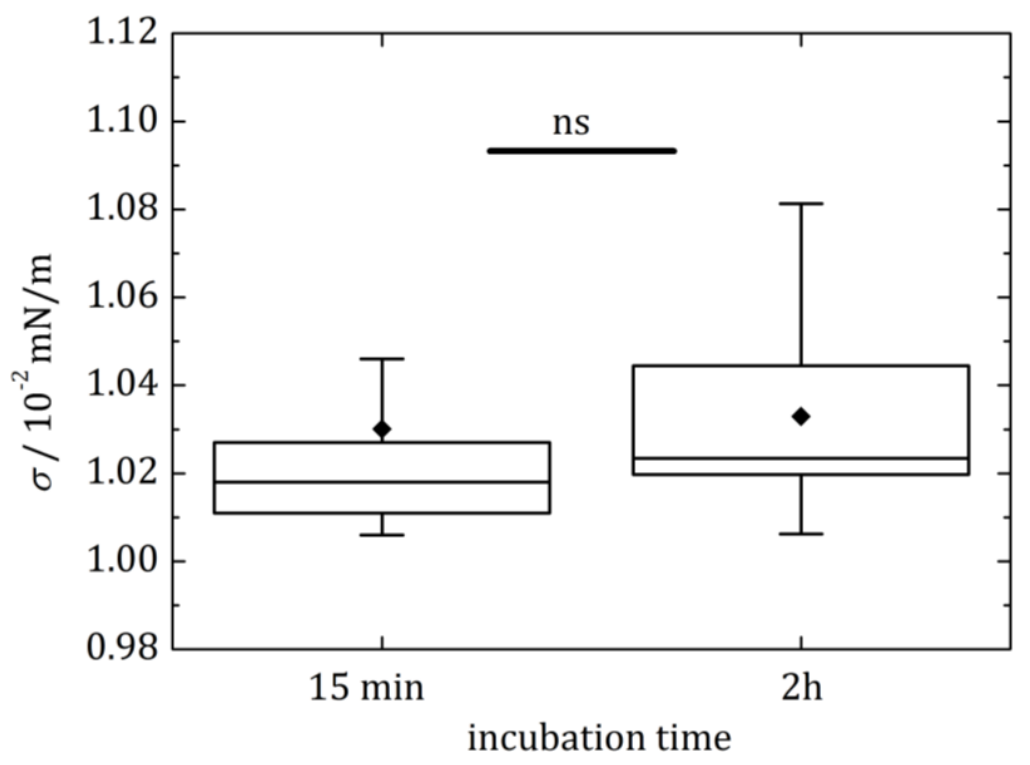

Figure 4.34: Lateral membrane tension of adhered GUVs on PEG-biotin-NeutrAvidin functionalized glass substrates after $15 \mathrm{~min}$ and $2 \mathrm{~h}$, showing that the membrane tension did not change during that time. Lipid composition: DOPC/DOPS/Cholesterol/cap-biotin-DOPE/PIP2/Atto488-DPPE (76:10:10:2:1:1). Statistical Mann-Whitney $U$ test: ns - not significant $(p=0.2)$.

The lateral membrane tension of the adhered GUVs after subsidence was $\sigma=(1.03 \pm 0.04) \cdot 10^{-2} \mathrm{mN} / \mathrm{m}$ and after $2 \mathrm{~h}$ the membrane tension of the GUVs was determined with $\sigma=(1.03 \pm 0.03) \cdot 10^{-2} \mathrm{mN} / \mathrm{m}$. The membrane tension of the GUVs did not changed significantly, indicating that the GUVs remained stable over time with quite low lateral membrane tensions of the adhered GUVs.

Since the regulation of the lateral membrane tension of the GUVs was not able with $\mathrm{MgCl}_{2}$ as it was previously done in the literature,36 the ratio of m-PEG to b-PEG on the substrate surface was varied to verify, if the membrane tension can be controlled by this. Changing the ratio of m-PEG/b-PEG from $1: 1$ to $1: 3$ did not change $r_{\mathrm{i}} / r_{\mathrm{ad}}$ of the vesicles (Figure 4.35). Hence, it was not possible to regulate the lateral membrane tension with the surface functionalization (Figure 4.36). 


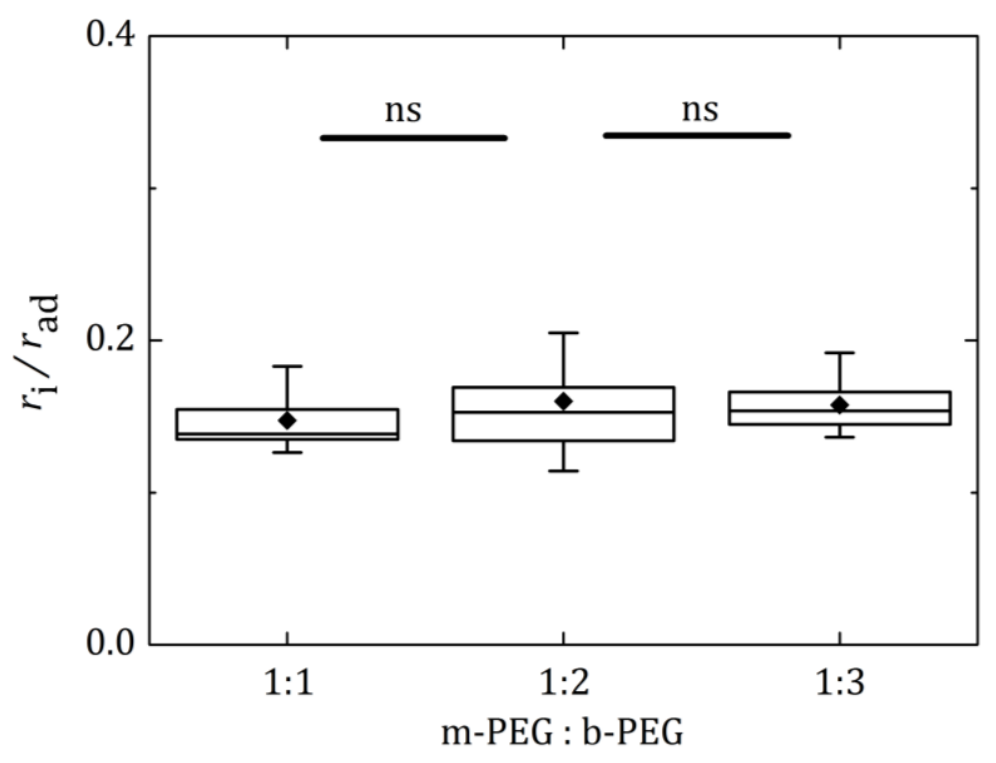

Figure 4.35: Ratio of the contact area radius and the adhered vesicle radius $\left(r_{\mathrm{i}} / r_{\mathrm{ad}}\right)$ of the adhered GUVs (DOPC/DOPS/Cholesterol/cap-biotin-DOPE/PIP2/Atto488-DPPE (76:10:10:2:1:1)) immobilized on PEG-Neutravidin functionalized glass with different ratios of b-PEG and m-PEG. Statistical Mann-Whitney $U$ test: ns - not significant ( $p>0.3)$.

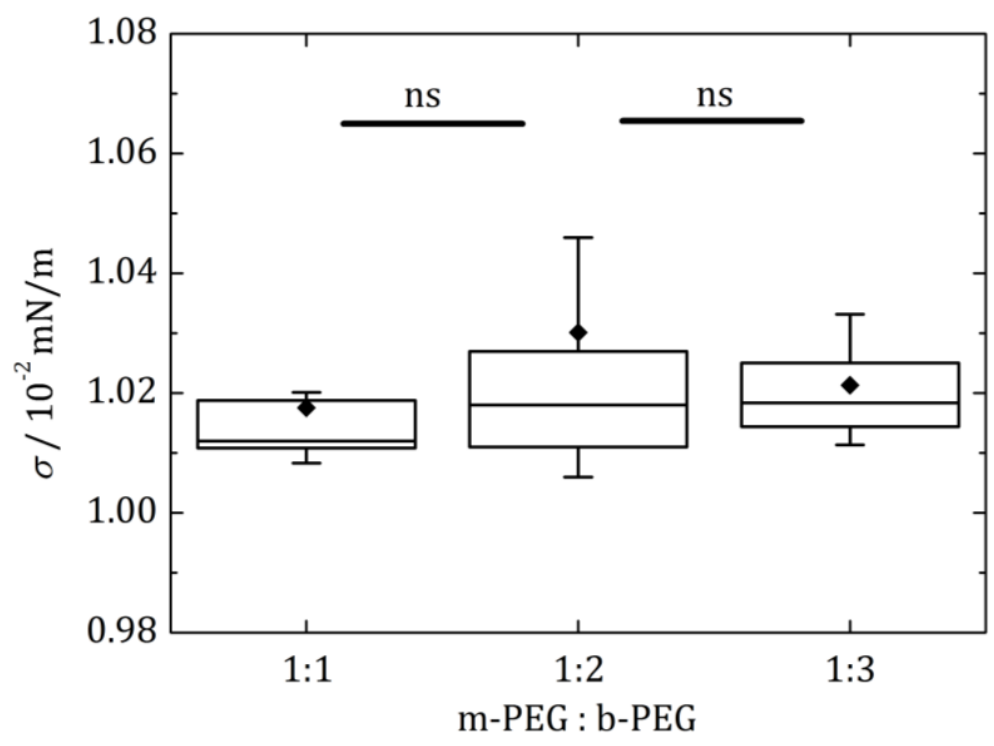

Figure 4.36: Lateral membrane tension of adhered GUVs on PEG-NeutrAvidin functionalized glass substrates with different ratios of m-PEG and b-PEG used for immobilization of the GUVs (DOPC/DOPS/Cholesterol/cap-biotin-DOPE/PIP2/Atto488-DPPE (76:10:10:2:1:1)). This shows that the membrane tension did not change by varying the PEG ratio. Statistical Mann-Whitney $U$ test: ns - not significant $(p>0.2)$. 
For each surface functionalization 16-25 GUVs were evaluated. The membrane tensions were determined to be $(1.02 \pm 0.02) \cdot 10^{-2} \mathrm{mN} / \mathrm{m}$ (m-PEG/b-PEG 1:1), $(1.03 \pm 0.04) \cdot 10^{-2} \mathrm{mN} / \mathrm{m} \quad(\mathrm{m}-\mathrm{PEG} / \mathrm{b}-\mathrm{PEG} \quad 1: 2)$ and $(1.02 \pm 0.01) \cdot 10^{-2} \mathrm{mN} / \mathrm{m}$ (m-PEG/b-PEG 1:3). Although the membrane tension of the adhered GUVs could not be regulated by the surface functionalization, they showed stability over $2 \mathrm{~h}$ under iso-osmolar conditions. Therefore, ENTH was added to investigate the effect of the protein binding on the membrane tension.

\subsubsection{Effect of ENTH addition to adhered GUVs}

Since the ratio of b-PEG and m-PEG showed no influence on the membrane tension of the adhered GUs, the following experiments were done with a ratio of 2:1 (b-PEG/mPEG). Moreover, it already was shown that with this ratio the PEG surface was covered homogenously with NeutrAvidin.

Adhered GUVs composed of DOPC/DOPS/Cholesterol/cap-biotinDOPE/PIP2/Atto488-DPPE (76:10:10:2:1:1) were incubated with $1 \mu \mathrm{M}$ ENTH for $2 \mathrm{~h}$. Every 30 min the solution was stirred to ensure a homogenous distribution of the protein. Afterwards, z-stacks of the ENTH incubated adhered GUVs were taken. Even though the amount of GUVs in the solution was not counted, it was obvious that less GUVs were found in the solution. Furthermore, a lot of lipid material was observed. Additionally imaging of the GUVs showed the rupture of the vesicles (Figure 4.37), indicating that the protein affects the vesicle stability. 

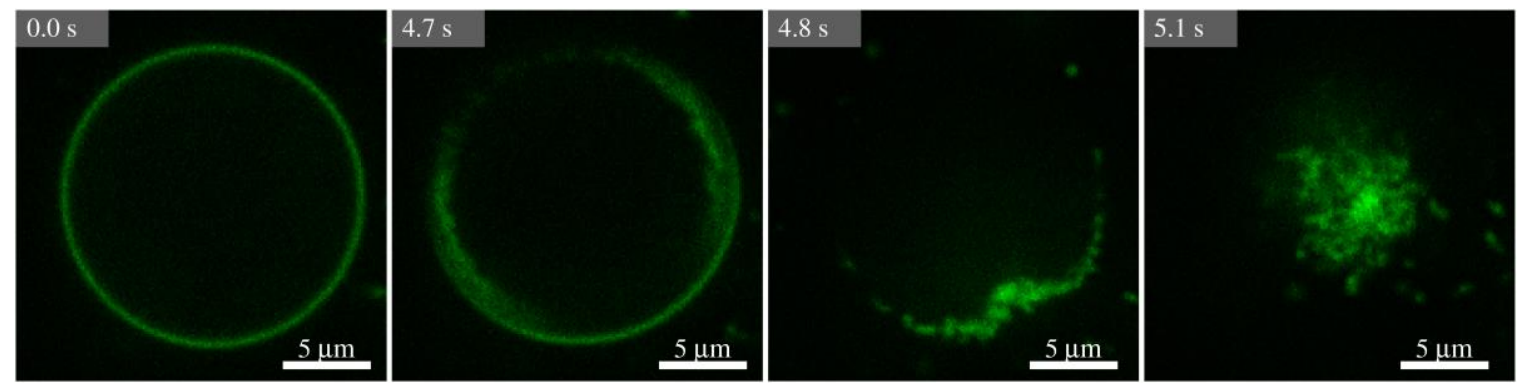

Figure 4.37: Time series of one z-plane of an adhered GUV incubated with $1 \mu \mathrm{M}$ ENTH (2 h). During imaging the vesicle ruptures. Lipid composition: DOPC/DOPS/Cholesterol/cap-biotinDOPE/PIP $2 /$ Atto488-DPPE (76:10:10:2:1:1).

Since the stability of the adhered GUVs without ENTH addition over $2 \mathrm{~h}$ was proven (Figure 4.33), rupturing of the GUVs seemed to be an effect of the ENTH binding to the PIP2 doped GUVs. In order to investigate if lower concentration still affects the GUV stability, the ENTH concentration was decreased to $0.3 \mu \mathrm{M}$. Even at this ENTH concentration the adhered GUVs ruptured during imaging (Figure 4.38). Decreasing the laser intensity did not change this as well.
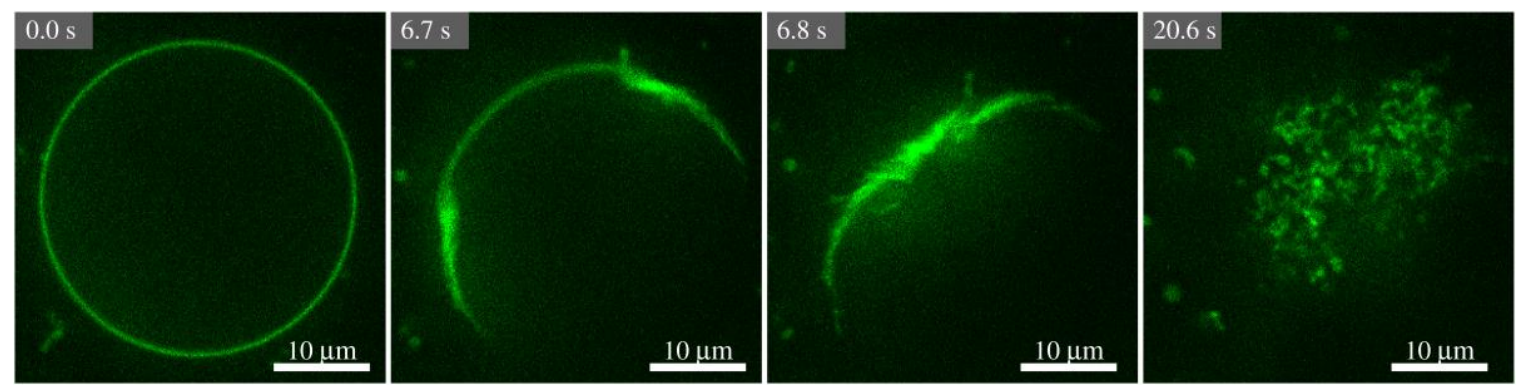

Figure 4.38: Time series of one z-plane of an adhered GUV incubated with $0.3 \mu \mathrm{M}$ ENTH ( $2 \mathrm{~h}$ ). During imaging the vesicle ruptures. Lipid composition: DOPC/DOPS/Cholesterol/cap-biotinDOPE/PIP2/Atto488-DPPE (76:10:10:2:1:1).

These results showed that the adhered GUVs ruptured during incubation with $1 \mu \mathrm{M}$ and $0.3 \mu \mathrm{M}$ ENTH, indicating that the ENTH helix insertion induce the destabilization of adhered GUVs. 



\section{Discussion}

To understand how the lipid composition influences the binding behavior of the epsin $N$-terminal homology (ENTH) domain different artificial membrane models and techniques were utilized. Reflectometric interference spectroscopy (RIfS) measurements allow to analyze the adsorption of ENTH and its dissociation constant dependening on the lipid composition. Using atomic force microscopy (AFM) the topology of the membrane surfaces with and without ENTH was analyzed. Moreover, the lipid dependent ENTH-PIP2 interaction was analyzed by Langmuir-Blodgett trough experiments and fluorescence microscopy. Monolayer penetration experiments allowed to obtain information about the insertion capability of the protein into a monolayer with different lipid compositions by detecting surface pressure changes. Protein activity as a function of membrane tension was investigated on adhered GUVs. In this chapter the results described in chapter 4 were compared to the literature and discussed in more detail.

\subsection{Characterization of the ENTH binding to PIP $_{2}$ containing solid supported membranes}

Phosphatidylinositol-(4,5)-bisphosphate $\left(\mathrm{PIP}_{2}\right)$ is the specific receptor lipid for the ENTH domain and consequently involved in the initial step of clathrin-mediated endocytosis (cf. chapter 1.1). In literature, a 1:1 binding of ENTH to $\mathrm{PIP}_{2}$ is described, which would predict a direct correlation of the amount of bound protein with the receptor concentration. ${ }^{7}$ Stahelin et al. already showed that at low $\mathrm{PIP}_{2}$ concentration $(0.5 \%)$ the dissociation constant drastically increases compared to higher $\mathrm{PIP}_{2}$ content (3\%). ${ }^{25}$ However, an influence of the PIP 2 concentration on the ENTH-membrane interaction was not investigated systematically yet and would clarify the relevance of its amount in the cytosolic bilayer leaflet. 


\subsubsection{Asymmetric distribution of PIP 2 in solid supported lipid bilayers and the influence on ENTH binding}

The binding behavior of ENTH to solid supported lipid bi- and monolayers was analyzed using RIfS. To measure the influence of $\mathrm{PIP}_{2}$ on the protein adsorption, planar and defect-free mono- and bilayers were required. For a pure POPC bilayer an optical thickness of $\triangle O T=6.4 \pm 0.2 \mathrm{~nm}$ was determined, which can be translated into a physical membrane thickness of $d \mathrm{M}=4.4 \pm 0.2 \mathrm{~nm}$ (equation (3.7)). Kŭcerke et al. measured a physical thickness of $d=3.98 \pm 0.08 \mathrm{~nm}$ for a POPC lipid bilayer. ${ }^{88}$ Other studies revealed membrane thicknesses of 3.68-4.60 nm, ${ }^{130-132}$ which are in agreement with the determined physical thickness of the POPC bilayer in this work.

Addition of $\mathrm{PIP}_{2}$ did not change the thickness, ranging from 4.3 to $4.5 \mathrm{~nm}$ without a correlation to the $\mathrm{PIP}_{2}$ content. The size of the $\mathrm{PIP}_{2}$ head group implies that it protrudes into the aqueous area when the structure is erected. ${ }^{44,98}$ These differences probably get lost in the detection range or in the errors of the measurements. Furthermore a possible hydration of the $\mathrm{PIP}_{2}$ head group would change the refractive index. An increased refractive index abolishes its exposed character and impedes its detection (cf. equation (3.7)). However hydration of lipid head groups already was shown to decrease the refractive index from $\sim 1.47$ to the refractive index of water $(n=1.33)$. Thus a limited detection range and the errors of the measurements seems more likely to detect significant changes of POPC and POPC/PIP 2 membranes. ${ }^{133}$

Physical thicknesses of POPC/PIP2 bilayers were previously measured via RIfS under the same conditions showing values in the same range. ${ }^{79,134}$ This confirmed the successful formation of POPC and POPC/PIP 2 bilayers.

On hydrophobic functionalized $\mathrm{SiO}_{2}$ wafers spreading of SUVs resulted in monolayers (cf. chapter 4.1.2). For a POPC monolayer the physical thickness of $d_{\mathrm{M}}=1.6 \pm 0.2 \mathrm{~nm}$ was determined. With increased $\mathrm{PIP}_{2}$ concentration the $d_{\mathrm{M}}$-values stayed approximately constant within the error margin. The physical thicknesses varied from 1.4 to $1.8 \mathrm{~nm}$, but no tendency considering the $\mathrm{PIP}_{2}$ content was observed. Since $4 \mathrm{~nm}$ of layer thickness is typical for a bilayer, monolayer thicknesses of about $2 \mathrm{~nm}$ are expected. ${ }^{88,130}$ Rossi et al. calculated an average thickness of $2.2 \pm 0.3 \mathrm{~nm}$ for an egg-PC monolayer by means of surface plasmon resonance spectroscopy (SPR). ${ }^{135}$ The values 
in this work are slightly smaller. Minor variations can be explained by the different techniques and surface functionalization procedures. In general the RIfS experiments revealed the successful formation of bi- and monolayers on hydrophilized and hydrophobic functionalized silicon surfaces, respectively.

Besides the RIfS experiments, fluorescence images were taken to prove the homogeneity and the mobility of the membranes. Therefore, the lipid bound fluorophore Texas $\operatorname{Red}^{\circledR}$ 1,2-dihexadecanoyl-sn-glycero-3-phosphoetanolamine (TxR) was used to stain the membrane. Spreading of SUVs on hydrophilic and hydrophobic functionalized $\mathrm{SiO}_{2}$ substrates resulted in bi- and monolayer formation, respectively. The fluorescence images of model membranes showed a predominant homogenous distribution of the fluorophore without significant defects. Fluorescence recovery after photobleaching (FRAP) experiments revealed that both systems were mobile, but the diffusion coefficients differed. For the POPC/TxR (99.5/0.5) and POPC/PIP2/TxR (99.5-x/x/0.5, with $\mathrm{x}=1,2,5,7,8$ and $10 \mathrm{~mol} \%$ ) bilayers diffusion coefficients of $D=1.8-2.5 \mu \mathrm{m}^{2} / \mathrm{s}$ were measured with the FRAP method. Compared to that, the monolayers exhibited diffusion coefficients from $D=0.1 \mu \mathrm{m}^{2} / \mathrm{s}$ to $0.4 \mu \mathrm{m}^{2} / \mathrm{s}$. In either case no correlation between the PIP2 content and the diffusion constant was observed. Aditionally, the diffusion constant decreased from bi- to monolayers by a factor of about 7 due to the interactions with the substrate. ${ }^{136}$ Between the bilayer and the substrate surface a thin water layer of about $1 \mathrm{~nm}$ exists, reducing the interaction with the substrate compared to monolayers. ${ }^{137-139}$

Braunger et al. determined diffusion coefficients of about $3 \mu \mathrm{m}^{2} / \mathrm{s}$ for both, bi- and monolayers using tetramethylrhodamine-labeled PIP $2 .{ }^{97}$ However, they used a dodecyl-trichlorosilane based functionalization for the monolayers. The silane might interact less with the $\mathrm{PIP}_{2}$ thereby explaining the same mobility of the fluorophore in bi- and monolayers. As they used labeled PIP 2 instead of TxR, the diffusion might also slightly differ. Furthermore, diffusion coefficients can be influenced by lipid composition, lipid packing, temperature and substrate preparation ${ }^{100,136,140-142}$ which could explain the differences. Baumann et al. measured diffusion coefficients of POPC/PIP2 lipid bilayers showing the influence of $\mathrm{PIP}_{2}$ on the mobility in the bottom leaflet. ${ }^{143}$ Measurements with labeled POPC (1-Palmitoyl-2-\{12[7-nitro-2-1,3-benzoxadiazol-4yl)amino]dodecanoyl\}-sn-glycero-3-phosphocholine, NBD-POPC) revealed diffusion 
constants of $D=1.84-2.46 \mu \mathrm{m}^{2} / \mathrm{s}$, comparable to the values observed in this thesis. They changed the $\mathrm{PIP}_{2}$ content from $1 \%$ to $10 \%$, which did not influence the mobility of NBD-POPC. Using labeled PIP2 did not change $D$, but the mobile fractions were significantly reduced, indicating the interactions of $\mathrm{PIP}_{2}$ with the substrate. Compared to that, the mobile fractions $\left(F_{\mathrm{m}}\right)$ of NBD-POPC did not change $\left(F_{\mathrm{m}}=84-91 \%\right)$ with varied PIP 2 content. In this work, the mobile fractions of TxR in the bilayers showed a slight decrease from $98 \pm 1 \%$ (2 mol\% $\left.\mathrm{PIP}_{2}\right)$ to $93 \pm 1$ (10 mol\% $\left.\mathrm{PIP}_{2}\right)$ in contrast to the monolayers with $F_{\mathrm{m}}=98 \pm 2 \%$ for all lipid compositions (cf. Table 4.2 ). The decreased mobile fractions with increased PIP2 concentration in the bilayers indicated a higher interaction of $\mathrm{PIP}_{2}$ with the hydrophilic substrate. The head groups of the bilayer are mainly interacting with the substrate. PIP 2 consists of a larger and highly negatively charged head group compared to POPC (Figure 5.1). Increasing the concentration hence can lead to an increased lipid-surface interaction compared to neutral lipids. ${ }^{144}$ In the monolayers the increase of $\mathrm{PIP}_{2}$ does not affect the mobility as only similar hydrocarbon chains were added.

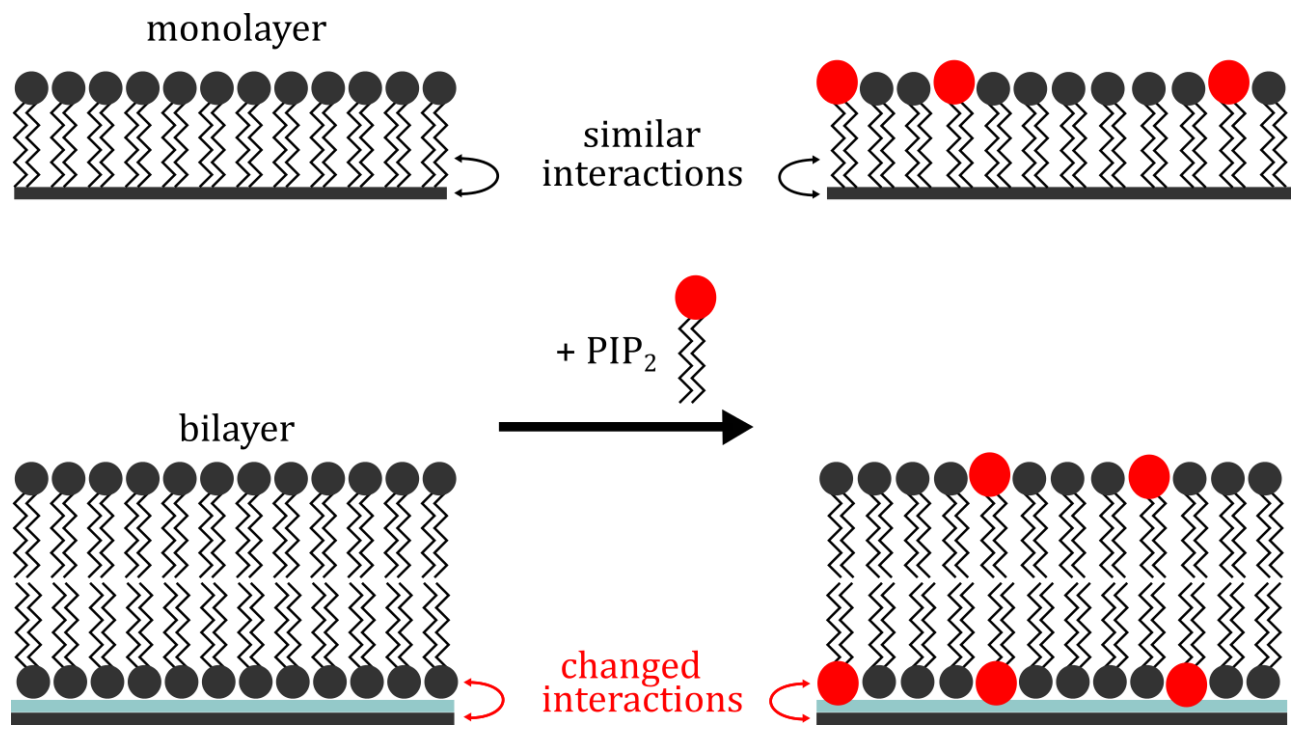

Figure 5.1: Schematic illustration of the different interactions of lipids with substrate surfaces. In the monolayers the increase of $\mathrm{PIP}_{2}$ does not affect the interactions between each other due to similar structures of the fatty acid chains. Compared to that increasing of the $\mathrm{PIP}_{2}$ content in bilayers results in increased interactions of the $\mathrm{PIP}_{2}$ lipid head groups. 
Baumann et al. also suggest that a larger hydration shell and charge repulsion of PIP2 result in the reduced bilayer mobility. They also assume that interactions of pits in the negatively charged substrate and the head group lead to a reduction of the mobile fraction. ${ }^{143}$

In this thesis the mobile fractions in the bilayers indicate a higher interaction between the substrate and the bottom leaflet with increased PIP2 concentration. This may influence the distribution of the receptor lipid within both leaflets of the membrane. Thus, the accessibility of PIP 2 for ENTH binding could be affected. Binding studies of ENTH to supported bilayers via RIfS were reported previously, ${ }^{36}$ but the influence of the $\mathrm{PIP}_{2}$ concentration is still not completely understood. For an exact analysis, the $\mathrm{PIP}_{2}$ molecules have to be entirely accessible. Therefore, besides the experiments on bilayers, also RIfS measurements were performed on monolayers to see if the substrate surface influences the $\mathrm{PIP}_{2}$ distribution.

\section{ENTH binding experiments}

ENTH binding experiments were performed in order to investigate the influence of the receptor lipid concentration on the binding affinity. Incubation of $1 \mu \mathrm{M}$ ENTH to pure POPC bi- and monolayers proved the specificity of the ENTH-PIP 2 interaction as no increase of the $\triangle O T$ was observed after protein addition (Figure 4.7). Itoh et al. revealed by co-sedimentation assays that only in the presence of $\mathrm{PIP}_{2}$ a high amount of ENTH binds to liposomes. Liposomes with other inositol species or negatively charged lipids showed a considerably decreased amount of bound protein or even no interaction. ${ }^{24}$ Kweon et al. moreover elucidated with electron paramagnetic resonance (EPR) studies the importance of $\mathrm{PIP}_{2}$ binding for the amphipathic helix formation. ${ }^{145}$

After the blind experiment with pure POPC, the binding of ENTH to $\mathrm{PIP}_{2}$-containing bilayers was analyzed. At low PIP 2 concentrations almost no ENTH binding was visible. An increase of the $\mathrm{PIP}_{2}$ content led to higher $\triangle O T$ values indicating an increased ENTH binding to $\mathrm{PIP}_{2}$-containing bilayers. Binding of ENTH to $\mathrm{PIP}_{2}$ and consequently conformational changes in the protein domain leads to the formation of a newly formed helix as well as a binding pocket for the receptor lipid. ${ }^{25}$ Based on this a 1:1 
binding of ENTH to PIP2 is assumed. This is in good agreement with the observed linear increase in $\triangle O T$ with rising $\mathrm{PIP}_{2}$ concentration (cf. Figure 4.9). Even at a $\mathrm{PIP}_{2}$ concentration of $10 \mathrm{~mol} \%$ and a $\triangle O T_{\mathrm{ENTH}}$ of $1.2+0.4 \mathrm{~nm}$ no obvious saturation of the curve was observed indicating an incomplete protein occupancy on the surface. Nevertheless, it should be considered that with the RIfS technique the data is averaged over an area of $1 \mathrm{~mm}^{2}$. Inhomogeneities hence can lead to underestimation of the physical thickness. Although a homogenous $\mathrm{PIP}_{2}$ distribution was observed by fluorescence microscopy previously, ${ }^{79,97,134}$ it is possible that nanoscopic clusters of $\mathrm{PIP}_{2}$ remained undetected. ${ }^{22}$ These clusters would prevent a 1:1 stoichiometry due to the size of the protein. Besides this, interactions of the lipids with the surface can influence the protein binding (cf. Figure 5.1).136 Gleisner achieved full coverage of ENTH on POPC/PIP2 (90:10) bilayers at a protein concentration of $3 \mu \mathrm{M}$ using RIfS. At $1 \mu \mathrm{M}$ a $\triangle O T$ of about $1.2 \mathrm{~nm}$ was determined, ${ }^{79}$ thus the results in this work are consistent with the literature. As the values were obtained via RIfS, they should be comparable to the data in this thesis (for $c\left(\mathrm{PIP}_{2}\right)=10 \mathrm{~mol} \%$ ) shown in Figure 4.9. Considering the crystal structure 22 and a maximum coverage of proteins (56\%) according to the scaled particle theory, ${ }^{146}$ a full coverage should be achieved at about $\Delta O T_{\mathrm{ENTH}}=2.0-2.8 \mathrm{~nm}\left(d_{\mathrm{ENTH}}=1.4-2.0 \mathrm{~nm}\right)$, dependent on the orientation of the protein on the surface. The crystal structure of the bound ENTH state is only known in the presence of the head group of $\mathrm{PIP}_{2}$, the inositol-1,4,5-triphosphate (IP 3 ). However, only binding to a membrane results in the formation of a stable amphipathic helix of ENTH $^{145}$ and consequently can also change the insertion and protein height. Moreover, interactions of ENTH and PS also can lead to an altered protein structure.

A full coverage of the protein was not yet reached at $10 \mathrm{~mol} \% \mathrm{PIP}_{2}$ in a bilayer. $\mathrm{PIP}_{2}$ has an area per molecule of about $0.7 \mathrm{~nm}^{2}$. With respect to the proteins footprint (about $16 \mathrm{~nm}^{2}$ ) 22,30 even in the presence of some small clusters, the surface would be completely covered at $10 \mathrm{~mol} \% \mathrm{PIP}_{2}$. This in turn shows that a certain amount of PIP2 seems to be inaccessible. The FRAP experiments on bi- and monolayers, discussed in the previous section, also indicated the uneven distribution of $\mathrm{PIP}_{2}$ in both leaflets of the bilayer. 
$\mathrm{PIP}_{2}$ exhibits a larger head group than POPC and was shown to have an exposed position in the membrane. ${ }^{44,147}$ Hence a high curvature in the SUV might provoke its location in the outer leaflet of the SUV. After spreading, the outer leaflet of the SUV is facing to the surface, ${ }^{148}$ resulting in the accumulation of $\mathrm{PIP}_{2}$ in the bottom leaflet. This would support the assumption that some PIP 2 molecules are inaccessible for ENTH binding. In monolayers this asymmetric distribution of $\mathrm{PIP}_{2}$ is prevented due to the missing second leaflet. For this reason ENTH binding experiments were repeated on PIP2-doped monolayers.

Binding studies of ENTH to POPC/PIP2 monolayers showed an increase of the optical thickness with increased lipid receptor concentration from $\Delta O T_{\mathrm{ENTH}}=0.5 \pm 0.1 \mathrm{~nm}$ $\left(1 \mathrm{~mol} \% \mathrm{PIP}_{2}\right)$ to $\triangle O T_{\mathrm{ENTH}}=2.2 \pm 0.4 \mathrm{~nm}\left(10 \mathrm{~mol}_{\mathrm{P}} \mathrm{PIP}_{2}\right)$. Compared to bilayers the $\triangle O T_{\text {ENTH }}$ values were about twice as high, indicating that $\mathrm{PIP}_{2}$ is asymmetrically distributed between both leaflets of a silicon supported bilayer.

In Figure 5.2 a schematic illustration of the predicted protein occupancy on bi- and monolayers due to $\mathrm{PIP}_{2}$ asymmetry is shown.
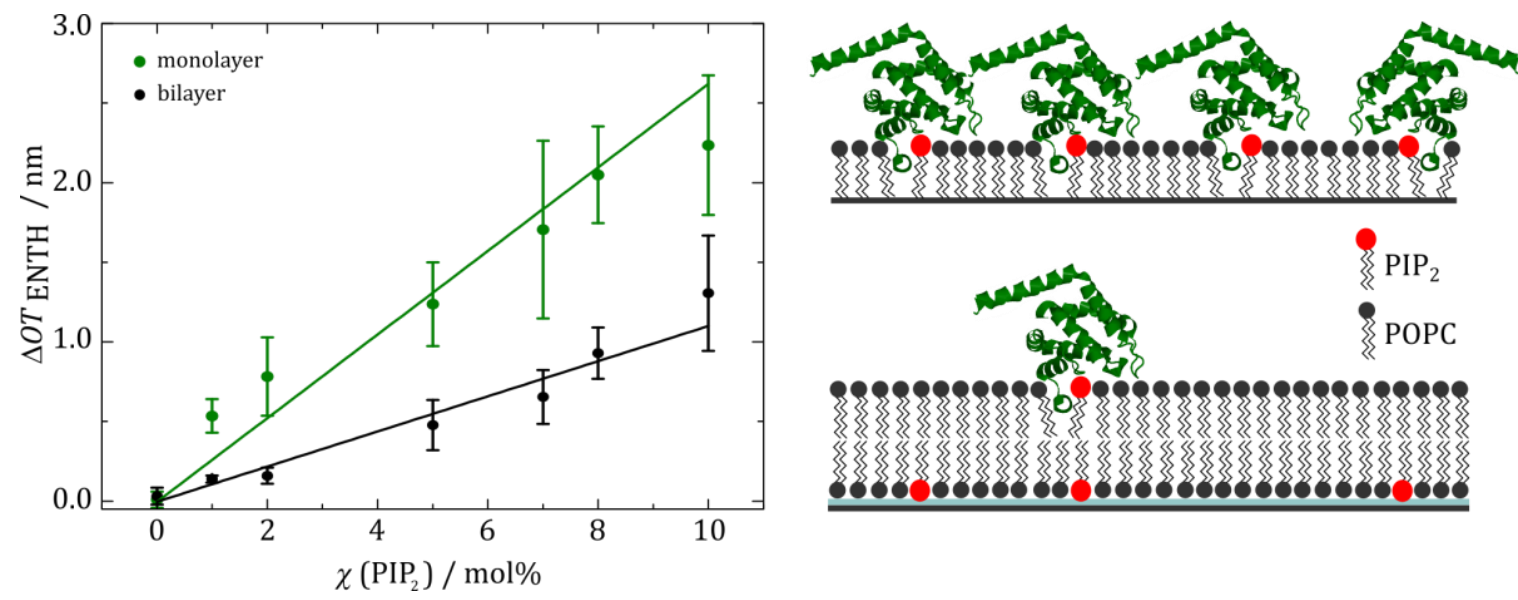

Figure 5.2: (A) Maximum change of the optical thickness upon ENTH binding to $\mathrm{PIP}_{2}$ doped mono(green) and bilayers (black) dependent on the PIP 2 content. (B) Schematic illustration of the $\mathrm{PIP}_{2}$ distribution within the mono- and bilayers and the influence on protein occupancy.

As already mentioned a full protein coverage on the membrane surface is expected at $\Delta O T_{\mathrm{ENTH}}$ values of $2.0-2.8 \mathrm{~nm} .{ }^{22,146}$ For the monolayers $\Delta O T_{\mathrm{ENTH}}$ was measured to be $2.2 \pm 0.4 \mathrm{~nm}$. Although the results (Figure 5.2) show no obvious saturation of the pro- 
tein occupancy at $10 \mathrm{~mol} \% \mathrm{PIP}_{2}$, a linear fit through the data indicates a slight overestimation of the expected $\triangle O T_{\mathrm{ENTH}}$ value at this $\mathrm{PIP}_{2}$ concentration. Within the error margins a saturation at 8 or $10 \mathrm{~mol} \% \mathrm{PIP}_{2}$ cannot be excluded, thus together with the achieved $\triangle O T_{\text {ENTH }}$ value an almost full coverage of ENTH on the monolayer can be implied.

In both membrane systems, bi- and monolayer, a high reversibility of the protein binding was observed. For the bilayer $87 \pm 24 \%$ and for the monolayer $75 \pm 14 \%$ of the protein bound reversibly. A high reversibility of the ENTH binding ensures the dissociation from the clathrin-coated vesicles (CCV, cf. chapter 1.1) during CME, with the result that recycled proteins are available for the next circle of the process. ${ }^{1,12}$ Hence, the obtained high reversibility in the RIfS experiments are reasonable with regard to the process occurring in biological membranes.

The results show that the amount of bound protein is highly regulated by the $\mathrm{PIP}_{2}$ concentration. A linear correlation between the protein occupancy and the $\mathrm{PIP}_{2}$ content furthermore support the assumed 1:1 binding of ENTH to PIP2 due to the formation of a $\mathrm{PIP}_{2}$ binding pocket within the protein.

\subsubsection{PS dependent binding affinity of ENTH to lipid bilayers}

Besides $\mathrm{PIP}_{2}$ also PS seems to have an influence on the ENTH-membrane interaction. ${ }^{8,26}$ This effect was also investigated by means of RIfS. Since the addition of other lipids can change the membrane properties, the formation of POPC/POPS (80:20) and POPC/POPS/PIP2 (75:20:5) bilayers was analyzed. Membrane thicknesses of $d=4.2 \pm 0.1 \mathrm{~nm}$ and $d=4.3 \pm 0.2 \mathrm{~nm}$ were measured for membranes with and without PIP2, respectively. Experiments with POPC and POPC/PIP2 (95:5) lipid compositions exhibited membrane thicknesses of $d=4.4 \pm 0.2 \mathrm{~nm}$ and $d=4.5 \pm 0.3 \mathrm{~nm}$ showing that there was no influence of the PS lipid head group on the membrane thickness. POPC/POPS (75:25) bilayer thicknesses of $3.81 \pm 0.60 \mathrm{~nm}$ were previously measured using dual polarization interferometry. ${ }^{149}$ The values in this work are similar to those within the error margins indicating the successful formation of a bilayer. 
Furthermore, fluorescence images showed a homogenous distribution of TxR in the POPC/POPS (80:20) and POPC/POPS/PIP2 (75:20:5) bilayers. Using FRAP, diffusion coefficients of $D=1.9 \pm 0.3 \mu \mathrm{m}^{2} / \mathrm{s}$ (without PS) and $D=1.3 \pm 0.4 \mu \mathrm{m}^{2} / \mathrm{s}$ (with PS) were determined. Increasing the negative charge decreased the mobility, but considering the error margins the differences were negligible. The same trend is observed for the mobile fractions. Without PS the mobile fraction of TxR in the bilayers was $F_{\mathrm{m}}=86 \pm 8 \%$. Increasing the negative charge with PS decreased the mobile fraction to $F_{\mathrm{m}}=77 \pm 4 \%$.

Zhang et al. measured the diffusion coefficient of fluorescent probes with different net charges in biofilms. The negatively charged probes diffused more slowly, which was ascribed to the electrostatic repulsion of the probes with the biofilms. ${ }^{144}$ Electrostatic repulsion of the negative charges $\mathrm{PIP}_{2}$ and PS also can affect the mobility of TxR in the bilayers.

In conclusion, mobile and homogenous bilayers composed of POPC/POPS (80:20) and POPC/POPS/PIP2 (75:20:5) were generated, enabling the investigation of the ENTH adsorption to these bilayers.

\section{Influence of PS on ENTH binding}

In order to investigate if ENTH binds specifically to PIP $_{2}$, ENTH was added to POPC/POPS (80:20) bilayers lacking PIP2, showing no binding of ENTH to PS. Hom et al. performed liposome binding assays with POPC/POPE/PIP2 (75:20:5) vesicles and showed specific binding of the ENTH domain to $\mathrm{PIP}_{2} .^{8}$ In the absence of $\mathrm{PIP}_{2}$ the domain did not associate with the vesicles, even though PS was present. These results were also supported by co-sedimentation assays performed by Itoh et al. No binding of ENTH to negatively charged lipids, like phosphatidic acid (PA), was observed. Furthermore less or even no binding of ENTH occurred to other phosphatidylinositol species. $^{24}$

The results in this thesis showed that the dissociation constant of ENTH to PIP 2 in the presence of PS decreased from $K_{\mathrm{D}}=1.0 \pm 0.2 \mu \mathrm{M}$ (POPC/PIP2 (95:5)) to $K_{\mathrm{D}}=0.42 \pm 0.05 \mu \mathrm{M}$ (POPC/POPS/PIP2 (75:20:5)). Moreover a higher protein occupancy was observed when PS was present $\left(\triangle O T_{\mathrm{ENTH}, \max }=2.5 \pm 0.1 \mathrm{~nm}\right.$ compared to 
$1.6 \pm 0.1 \mathrm{~nm}$ ). This indicates that PIP2 is necessary for the binding of ENTH to membranes, however PS decreased the dissociation constant of ENTH to $\mathrm{PIP}_{2}$ doped bilayers and increased the protein occupancy on them.

Gleisner et al. measured $K_{\mathrm{D}}$ values by means of SPR, RIfS and spinning disc confocal laser microscopy (SDCLM). ${ }^{36,79}$ RIfS experiments revealed a $K_{\mathrm{D}}$ of $0.8 \pm 0.8 \mu \mathrm{M}$ for POPC/PIP2 (90:10). The KD value is similar to the value obtained in this thesis via RIfS. With densitometry analysis of SDS-PAGE Hom et al. revealed that vesicles (diameter $\approx 1 \mu \mathrm{m})$ composed of POPC/POPE/POPS/PIP2 (63:20:15:2) bound more ENTH than POPC/POPE/PIP2 (78:20:2) vesicles indicating the influence of PS on the ENTH$\mathrm{PIP}_{2}$ interaction. ${ }^{8}$ With SPR experiments they furthermore measured $K_{\mathrm{D}}$ values of $20 \pm 2.4 \mathrm{nM}$ in presence of PS and $80 \pm 11 \mathrm{nM}$ when PS was absent, both at a pH of 7.4, mimicking the natural $\mathrm{pH}$ of mammalian cells. ${ }^{150}$ In this thesis the $K_{\mathrm{D}}$ values were larger than the $K_{\mathrm{D}}$ values obtained by Hom et al. ${ }^{8}$ Recruitment of hydrophobic domains to curved membranes is preferred due to the higher binding site density and thus might explain the differences in the binding affinity. ${ }^{151-153}$ However, Hom et al. observed that PS clearly enhanced the amount of bound ENTH to PIP2-containing membranes supporting the result obtained in this work. ${ }^{8}$ Addition of PS clearly increased the binding affinity of ENTH to $\mathrm{PIP}_{2}$-containing membranes (Figure 4.14).

Various other studies revealed values from 0.02 to $6 \mu \mathrm{M}$ using fluorescence microscopy, SPR or isothermal titration calorimetry (cf. Appendix, Table 10.3). ${ }^{8,21,25,28,36,79,154,155}$ The dissociation constants determined in this thesis are comparable to the values in literature.

PS is localized at the cytosolic side of mammalian plasma membranes, ${ }^{37}$ where ENTH binds to, ${ }^{156}$ hence a contribution to the protein-membrane interaction is theoretically conceivable. Stahelin et al. suggested that non-specific interactions of ENTH with negative charges recruit the ENTH to the membrane which enables binding of ENTH to $\mathrm{PIP}_{2 .}{ }^{25}$ Binding leads to conformational changes in the protein which also could explain that PS only has an effect on ENTH binding when $\mathrm{PIP}_{2}$ is present (Figure 4.13). Otherwise, in the absence of PIP2, no ENTH binding was observed. Thus, PS could enhance the recruitment and subsequently the binding affinity of ENTH. One explanation could be that due to ENTH-PIP2 interaction a binding site for PS gets accessible or non-specific interactions with positive protein residues facilitate the binding. Since 
in the presence of PS a higher protein occupancy on the bilayer was observed, the question arises if besides the helix insertion a crowding mechanism can contribute to a higher amount of bound protein in the presence of PS (Figure 5.3).

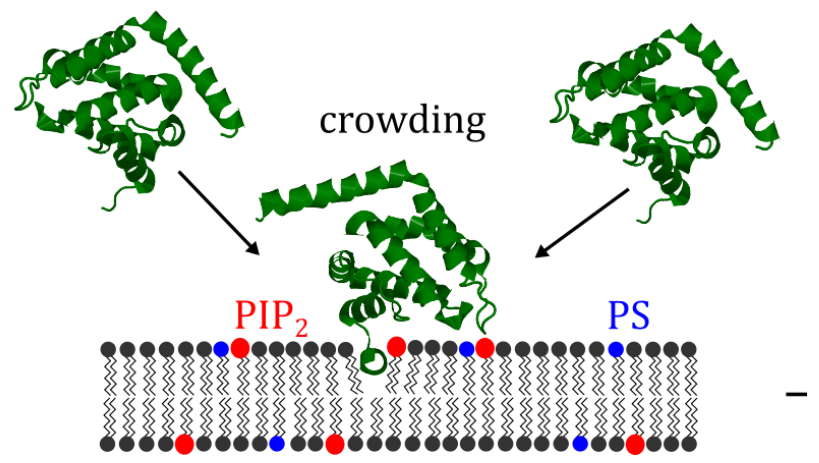

Figure 5.3: Schematic illustration of the ENTH crowding mechanism. The assembly of several ENTH monomers increases the protein occupancy on PS containing membranes in a PIP 2 dependent manner.

Kroppen showed with co-sedimentation assays that ENTH tends to form homo-oligomers in the presence of $\mathrm{PS}^{26}$ explaining the higher $\Delta O T_{\mathrm{ENTH}}$ max values (Figure 4.15). Protein-protein interactions can lead to higher surface occupancies or to stacked protein clusters. Both would increase the optical thickness. However, with RIfS experiments the formation of clusters cannot be observed.

\section{PS induced ENTH cluster formation}

Since PS showed an influence on the ENTH occupancy, AFM experiments were performed to obtain information about the surface topology after ENTH adsorption. Due to the comparability with the literature $26,36,151$ the lipid composition was changed to DOPC/DOPE/PIP $2 / T x R \quad$ (64.9:30:5:0.1) and DOPC/DOPE/DOPS/PIP2/TxR (44.9:30:20:5:0.1). AFM experiments to investigate protein adsorption were previosly performed with DOPC membranes, ${ }^{157,158}$ showing the applicability for this analysis.

Beforehand, FRAP experiments proved the mobility of these defect free membranes (Figure 4.19) with a $D=2.5 \pm 0.6 \mu \mathrm{m}^{2} / \mathrm{s}$ (without PS) and $D=1.7 \pm 0.4 \mu \mathrm{m}^{2} / \mathrm{s}$ (with PS), typical for bilayers composed of DOPC and DOPC/DOPS. ${ }^{159-161}$ The decreased $D$ value is explainable with the increased negative charges of PS in combination with 
$\mathrm{PIP}_{2}$ interacting with the hydrophilic surface. ${ }^{136,143}$ Breakthrough experiments additionally verified the formation of bilayers with a membrane thickness of about $4 \mathrm{~nm}$, indicating the successful formation of mobile bilayers. ${ }^{162}$

Incubation with $1 \mu \mathrm{M}$ ENTH led to a significant decrease of the membrane mobility resulting in diffusion constants of $D=0.6 \pm 0.2 \mu \mathrm{m}^{2} / \mathrm{s}$ and $D=0.3 \pm 0.2 \mu \mathrm{m}^{2} / \mathrm{s}$ in absence and in presence of PS, respectively. Reduction of the lateral lipid diffusion is often observed when proteins bind to membranes. ${ }^{110,163-165}$ Due to binding of proteins, lipids diffuse slower in a protein-lipid complex. Hence decreased diffusion of ENTH-PIP2 complexes can affect the lateral diffusion of TxR in the bilayers.

In the atomic force micrographs (Figure 4.17), structures with an average height of $1.2 \pm 0.2 \mathrm{~nm}$ were observed in the presence of PS indicating the adsorption of ENTH. The protein occupancy of $6 \pm 1 \%$ indicates that only a minor amount of the protein is detected by AFM. Furthermore, no protein clusters were observed in in the absence of PS. At first sight this contradicts the results obtained with RIfS. Although the lipids differ, the head groups are the same and consequently the occupancy should not be affected that much. Previous studies also revealed similar binding behaviors of ENTH to membrane systems composed of POPC or DOPC..$^{36,154}$

The low occupancy moreover could be explained by the high reversibility of the ENTH binding detected via RIfS (Table 10.2). During imaging in contact mode lateral shear forces from the tip occurring to the sample can harm or detach the protein. ${ }^{166}$ The movement of the cantilever over the sample, hence, could shift the ENTH over the sample (Figure 5.4). This would also explain that no proteins were observed on the membrane in the absence of PS. Thus only PS induced ENTH clusters, which were rigid enough to be scanned in contact mode, were clearly imaged, causing an underestimation of the protein occupancy. 

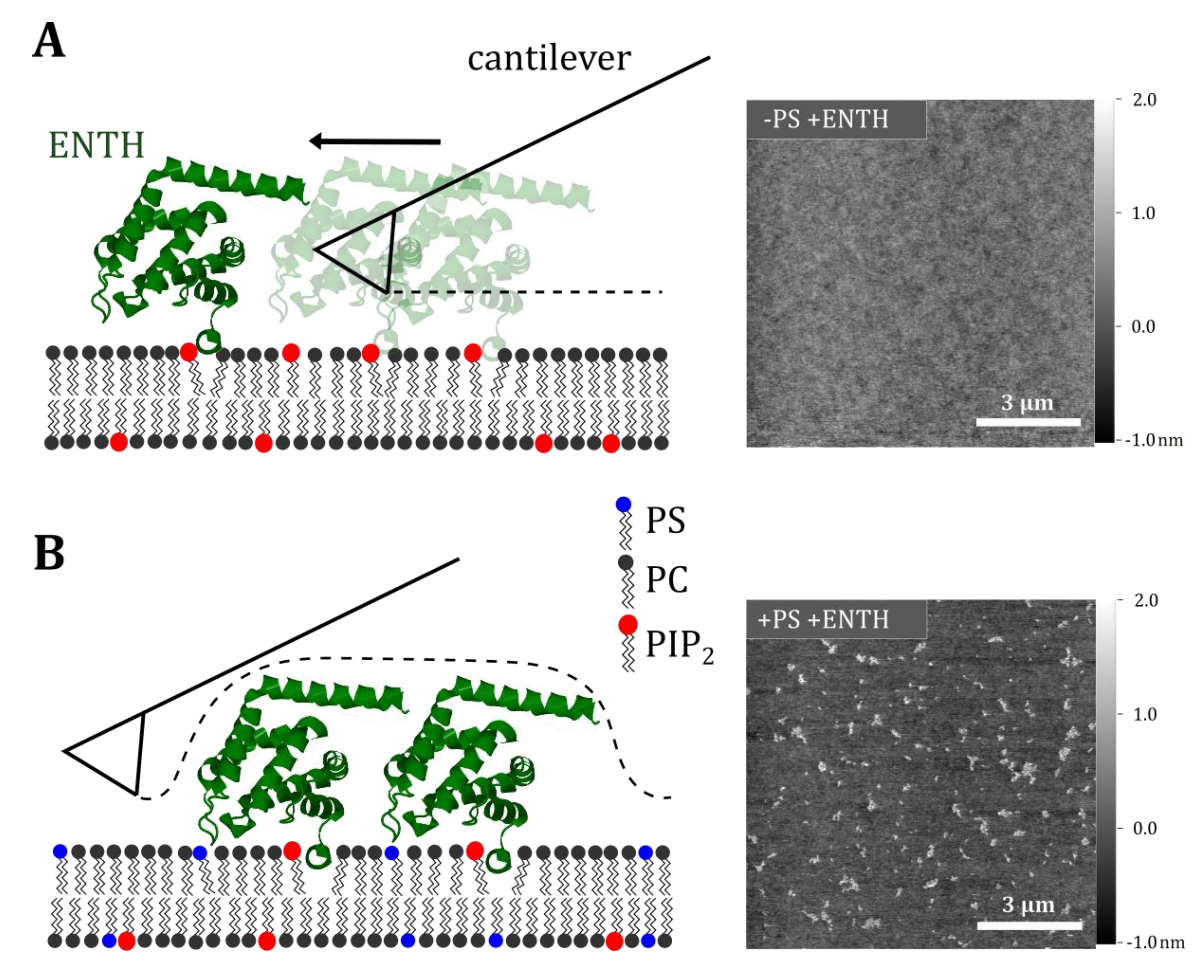

Figure 5.4: Cantilever movement in contact mode during imaging of (A) ENTH monomers and (B) ENTH clusters. Due to the mobile character of ENTH monomers the cantilever shifts the protein over the sample preventing the imaging of single monomers in contrast to immobile clusters.

EPR studies performed by Lai et al. indicated an immobilization of the protein due to close proximity of adjacent ENTH monomers, supporting the assumption that the aggregates in the micrographs are immobile and consequently only these were detected. ${ }^{110}$ They furthermore hypothesized that ENTH dimers or larger aggregates can occur dependent on the local membrane morphologies. Curvature facilitates the aggregation explaining the minor amount of visible clusters in the micrographs of planar solid supported bilayers.

Previous studies furthermore showed that ENTH tends to form homo-oligomers in the presence of PS. ${ }^{26}$ Exchanging PS against phosphatidylinositol (PI) to mimic the negative charge ${ }^{167}$ did not lead to oligomers. Hence, this supports the assumption that the cluster formation is induced by PS and not only by negative charges.

Although imaging in contact mode obtained low protein occupancies, other imaging modes were unsuitable for this system due to the appearance of inversion effects. ${ }^{168,169}$ Imaging in tapping mode yielded micrographs, where dark structures in the bilayers were detected. Micrographs obtained in contact mode however revealed 
that these dark structures were attributed to ENTH clusters and were a result of the inverted image contrast. In Figure 5.5 atomic force micrographs of a DOPC/DOPE/DOPS/PIP2/TxR (44.9:30:20:5:0.1) bilayer after incubation with $1 \mu \mathrm{M}$ ENTH obtained by QI-, tapping- and contact mode are illustrated. Rahe et al. observed inversion of the image contrast of protruded adsorbates on mica and $\mathrm{TiO}_{2}$ in tapping mode depending on the amplitude of the cantilever oscillation and the damping level of the cantilever. ${ }^{170}$ Changed attraction of cantilever and adsorbate caused a phase shift of driving force and driven oscillation and consequently incorrect readout of the height. Hence in this work the different interactions between the cantilever with the bilayer and the protein could have led to the inverted contrast in the micrograph during imaging in tapping mode and consequently prevented the analysis of protein heights with this imaging mode.
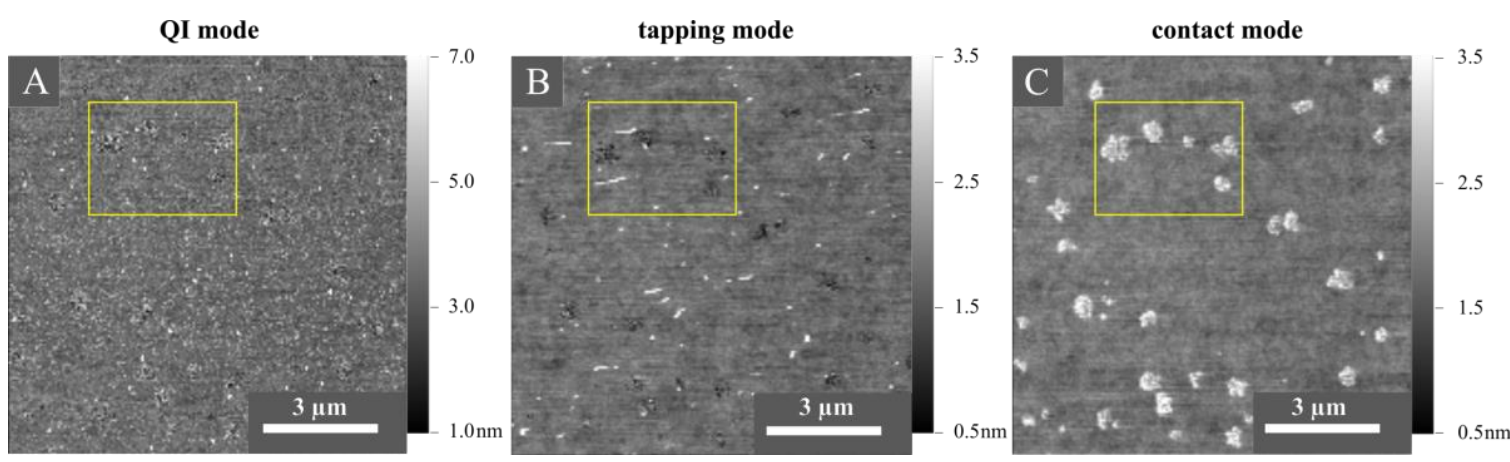

Figure 5.5: Atomic force micrographs of a DOPC/DOPE/DOPS/PIP $2 / T x R ~(44.9: 30: 20: 5: 0.1)$ bilayer after incubation with $1 \mu \mathrm{M}$ ENTH obtained by three different imaging modi: QI-, tapping and contact mode. The yellow squares highlight one part of the micrographs to underscore differences. Only when the measurements were performed in contact mode bright cluster structures were observed. Due to the inversion of the image contrast the ENTH clusters falsely were detected as membrane defects.

The atomic force micrographs obtained in contact mode (Figure 4.17 and Figure 5.5) clearly showed that PS induced the formation of ENTH cluster. The height of these clusters was determined to be $1.2 \pm 0.2 \mathrm{~nm}$ which is smaller than reported in literature. $^{22}$ Considering the crystal structure of ENTH at least a protein height of 2.0$2.8 \mathrm{~nm}$ was expected. Due to this, the bilayer surface was investigated by fluorescence microscopy to exclude ENTH binding to defects (Figure 4.19). Binding of ENTH to defects would lead to an underestimated protein height. To ensure that these defects 
moreover did not appear during the membrane aging process, the bilayers were incubated in buffer for two hours and then imaged again showing no defects. In all cases, with or without PS, no visible defects were observed before and after ENTH addition or in the absence of ENTH after $2 \mathrm{~h}$. Hence the low protein height is not resulting from binding to defects and consequently the clusters were attributed to protein adsorption to PIP2 doped membranes in the presence of PS.

The crystal structure of the bound ENTH is only known in the presence of inositol1,4,5-trisphosphate $\left(\mathrm{IP}_{3}\right)$ (head group of $\mathrm{PIP}_{2}$ ) and not when it is bound to the receptor lipid PIP2. Although binding of ENTH to IP3 alone results in the formation of the ENTH helix, it is not stable outside the membrane. When ENTH binds to a $\mathrm{PIP}_{2}$ doped membrane, the helix is stabilized which would facilitate its insertion into the inner leaflet (Figure 5.6). ${ }^{145}$

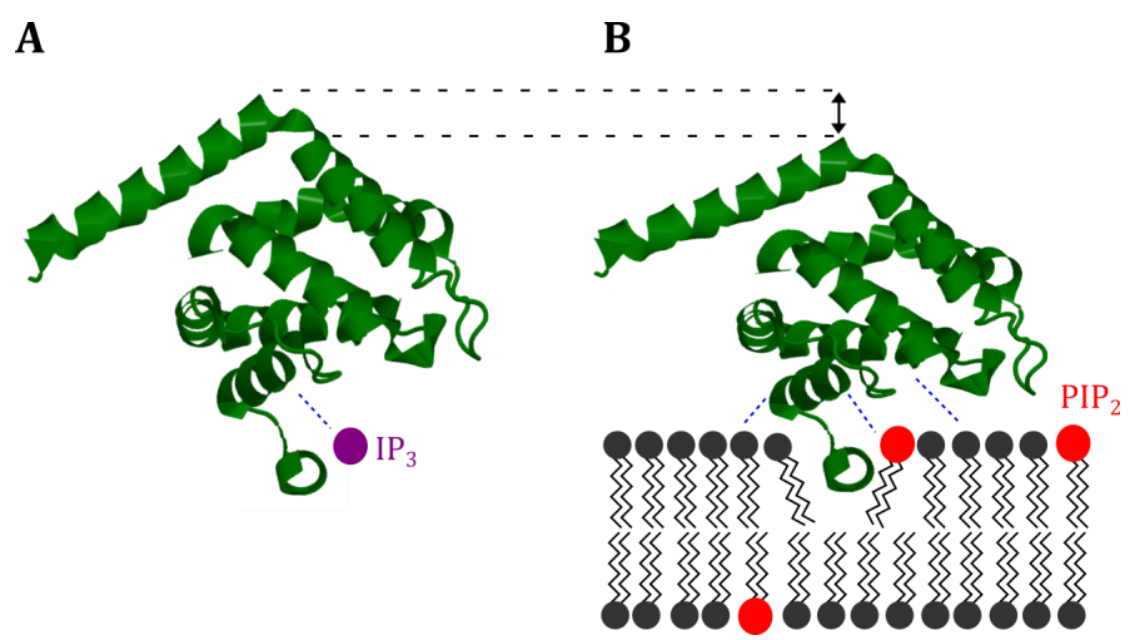

Figure 5.6: Schematic illustration of the different interactions of (A) IP 3 bound ENTH and ENTH bound to a PIP 2 doped membrane. Only when ENTH is bound to the membrane the helix is stabilized, which could lead to a slightly thinner protein layer on the bilayer surface compared to the assumed protein height from the crystal structure. ${ }^{21,145}$

This would lead to an underestimation of the detected protein height compared to the values evaluated from the crystal structure. However, previous studies determined the physical thickness of ENTH via RIfS, yielding $d_{\text {ENTH }}=1.7 \pm 0.2 \mathrm{~nm} .{ }^{79}$ Similar values were also obtained in this thesis by means of RIfS. Assuming a maximum of the protein height of $56 \%$ according to the scaled particle theory the actual height increases to $3.0 \mathrm{~nm} .{ }^{146,171}$ The differences in the protein heights are mainly explainable by the 
used techniques. The protein heights of $1.2 \pm 0.2 \mathrm{~nm}$ in this thesis were obtained by means of AFM in contact mode. During imaging in contact mode indentation of the protein with the cantilever can underestimate the protein height (Figure 5.7 A). ${ }^{166}$ Furthermore altered interactions of protein with the bilayer, e.g due to increase of the negative charge by PS, could affect the orientation and structure of the bound protein. This would result in a slightly different protein height compared to the protein height obtained from the crystal structure or with other techniques and lipid compositions (Figure 5.7 B and C). ${ }^{22,79}$ Also other proteins, like synaptotagmin-1, showed different orientations with respect to $\mathrm{PIP}_{2}$-containing membranes when PS is present. ${ }^{53}$ Since the presence of PS was shown to decrease the dissociation constant and increase the protein occupancy (cf. chapter 4.2.2) on the bilayer, altered interactions with the bilayer surface seems reasonable. Thus, the observed structures were attributed to ENTH clusters.

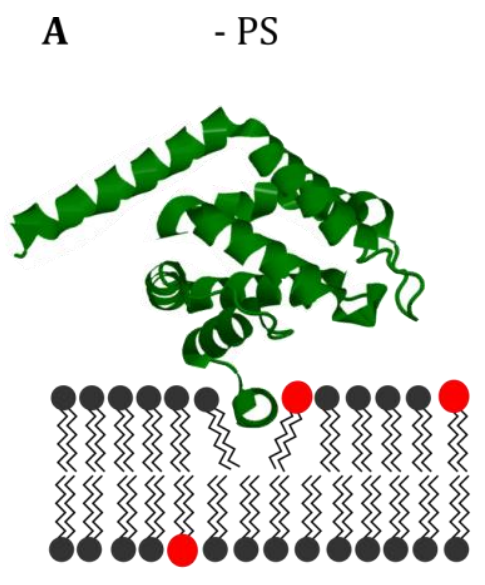

$\mathrm{PC}$
B $\quad+$ PS

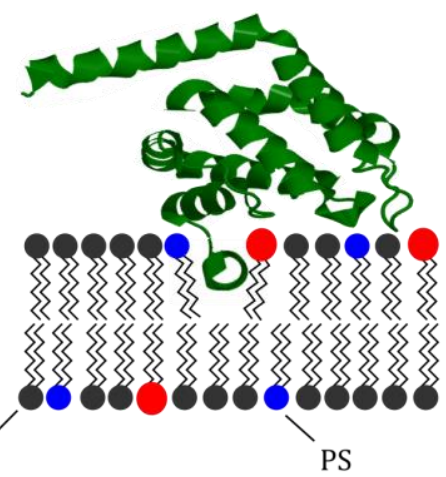

C $\quad+$ PS

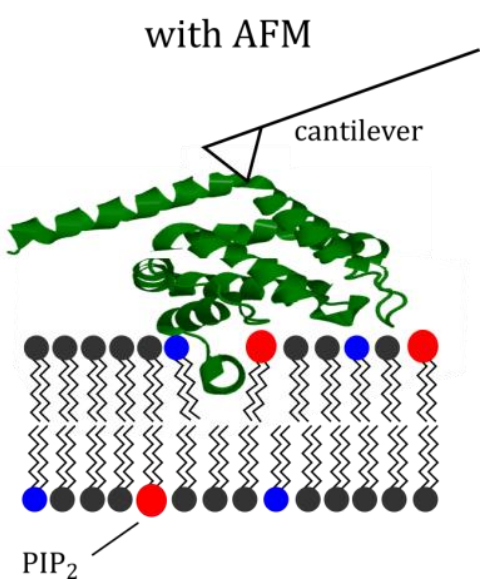

Figure 5.7: Predicted protein heights of ENTH for different conditions. (A) ENTH bound to PIP2-doped membranes. (B) ENTH bound membranes containing PIP 2 and PS. (C) Indentation of the ENTH bound to $\mathrm{PIP}_{2} / \mathrm{PS}$ membranes by the cantilever. Due to lipid dependent protein-membrane interactions the protein height can change. Moreover imaging in AFM can indent the protein resulting in the detection of a decreased protein height.

The results clearly show that PS has a significant impact on the binding behavior of ENTH to PIP2 doped membranes. First, the dissociation constant of ENTH to those bilayers is decreased and the protein occupancy on the surface increased. Moreover the AFM experiments revealed that PS induces the formation of ENTH clusters in the 
presence of $\mathrm{PIP}_{2}$. Hence, this shows that not only $\mathrm{PIP}_{2}$ regulates the recruitment of ENTH to the membrane, but also PS highly affects the binding structure of ENTH to membranes.

\subsubsection{The ENTH R114A mutant}

There are different regions within the ENTH domain involved in protein-protein and protein-lipid interactions. Especially the amino acid R114 was found to interact with the membrane and to be involved in the formation of ENTH homo-oligomers. ${ }^{26,110}$ Lai et al. performed coarse-grained molecular dynamics (CG-MD) simulation and EPR experiments with the ENTH domain. Their results indicated an interaction of the amino acid R114, located in the unstructured loop between helix 6 and helix 7, with the membrane. ${ }^{110}$ This interaction further recruits ENTH and stabilizes the ENTH membrane docking geometry. Hence, the amino acid R114 can be involved in the cluster formation of ENTH.

In order to investigate the role of the amino acid R114 in the formation of clusters, as observed in the presence of PS (Figure 4.17), RIfS and AFM experiments were performed with the mutant R114A. The results showed a decreased dissociation coefficient of the mutant on POPC/POPS/PIP2 bilayers and a higher protein occupancy on the membrane compared to bilayers lacking PS. Furthermore similar surface occupancies of ENTH R114A and ENTH wt were determined indicating that the mutant is still binding to $\mathrm{PIP}_{2}$ doped membranes. However, the dissociation constant of the mutant significantly increased compared to the wt showing the contribution of the amino acid R114 in the binding process of ENTH. The obtained values for the ENTH wt and mutant are listed in Table 5.1. 
Table 5.1: Summary of the fit results of the Langmuir adsorption isotherms for ENTH wt and ENTH $\mathrm{R} 114 \mathrm{~A}$. The values are given as parameter $\pm \mathrm{SE}$.

\begin{tabular}{|l|l|l|l|l|}
\hline \multirow{2}{*}{$\begin{array}{l}\text { lipid } \\
\text { composition }\end{array}$} & \multicolumn{2}{|c|}{$K_{\mathrm{D}}$} & \multicolumn{2}{c|}{$\Delta$ OT ENTH max } \\
\cline { 2 - 5 } & ENTH wt & ENTH R114A & ENTH wt & ENTH R114A \\
\hline $\begin{array}{l}\text { POPC/ PIP } 2 \\
(95: 5)\end{array}$ & $1.0 \pm 0.2 \mu \mathrm{M}$ & $2.9 \pm 0.8 \mu \mathrm{M}$ & $1.6 \pm 0.1 \mathrm{~nm}$ & $1.5 \pm 0.2 \mathrm{~nm}$ \\
\hline $\begin{array}{l}\text { POPC/POPS/PIP2 } \\
(75: 20: 5)\end{array}$ & $0.42 \pm 0.05 \mu \mathrm{M}$ & $1.0 \pm 0.3 \mu \mathrm{M}$ & $2.5 \pm 0.1 \mathrm{~nm}$ & $2.7 \pm 0.2 \mathrm{~nm}$ \\
\hline
\end{tabular}

In general, the dissociation constants lie within the same range as the values found in literature (cf. Appendix, Table 10.3). Stahelin et al. mutated the ENTH domain at different positions. Mutations, like L6Q and M10Q strongly affected the binding affinity and the penetration ability into monolayers as these amino acids were localized in the $\alpha_{0}$ helix. ${ }^{25}$ The R114A mutant in turn exhibited a decreased dissociation constant compared to the wt, but showed a similar penetration ability into POPC/POPE/PIP2 (77:20:3) as the ENTH wt. This amino acid is not located at the PIP 2 binding site, but the close contact to the membrane facilitates the contribution in non-specific electrostatic interactions. ${ }^{23,25,110}$

Lack of the positively charged protein residue (R114) or the negatively charged PS in the membrane led to a decreased binding affinity (Figure 4.21) and hence support the assumption that the amino acid R114 is involved in further recruitment of ENTH when PS was present. As the occupancy of ENTH R114A in presence of PS was similar to that of ENTH wt (cf. Table 5.1), it seems likely that still interactions of PS and the mutant occur in a $\mathrm{PIP}_{2}$ dependent manner, but ENTH clustering is prevented (Figure 4.22). Although the $K_{\mathrm{D}}$ of the mutant is higher as the value for the ENTH wt, the mobility of single ENTH R114A molecules seemed to be higher than the mobility of the ENTH wt clusters, hence the resolution of individual ENTH R114A monomers (and also individual ENTH wt monomers in the absence of PS, Figure 4.17) in contact mode was not possible.

Previous studies revealed the contribution of the amino acid R114 of ENTH in recruitment and membrane deformation. Kroppen showed that the mutation of this amino 
acid did not change the molecular structure of ENTH and still was binding to the membrane in presence of $\mathrm{PIP}_{2}$ and PS. ${ }^{26}$ However, the ability to form tubular structures got lost proving the importance of this amino acid. ${ }^{26}$ Moreover, co-sedimentation assays revealed the contribution of R114 in homo-oligomer formation. To investigate if the mutant also prevents cluster formation on solid-supported membranes, AFM experiments were performed. In all cases (DOPC/DOPE/PIP2/TxR (64.9:30:5:0.1) and DOPC/DOPE/DOPS/PIP2/TxR (44.9:30:20:5:0.1) homogenous and planar bilayers with a roughness of $152 \pm 7$ pm (without PS) and 197 \pm 47 pm (with PS) were observed after the incubation with ENTH R114A. Even in the presence of PS no clusters appeared (Figure 4.22) meaning that the lack of the arginine in position 114 prevented the formation of clusters. This also shows that the presence of PS and the amino acid R114 are required for the cluster formation which also can indicate an interaction of PS and R114.

Yoon et al. already revealed that interactions between two ENTH monomers can occur in the presence of $\mathrm{PIP}_{2}$ and PS. ${ }^{28}$ Although they did not investigate the impact of PS, they performed vesicle binding studies with GUVs composed of POPC/POPE/POPS/PIP2/Rh-PE (46.5:30:20:0.5, Rh-PE = 1,2-dipalmitoyl-sn-glycerol3-phosphoethanolamine- $N$-(lissamine rhodamine B sulfonyl)) showing the appearance of tubulation induced by ENTH crowding. This was attributed to the interaction of K23 and E42 of two ENTH monomers. When the amino acids were mutated (K23E and $\mathrm{E} 42 \mathrm{~K}$ ) the ENTH lost the ability to induce tubulations. Their vesicle binding studies were supported by EPR studies which indicated an interaction of two $\alpha_{0}$ helices (ENTH wt) in an antiparallel manner meaning a well-ordered clustering of the proteins. In combination with the results observed in this thesis, a contribution of PS and R114 in the accessibility of the amino acids K23 and E42 for protein-protein interactions (Figure 5.9) seems likely. 
A

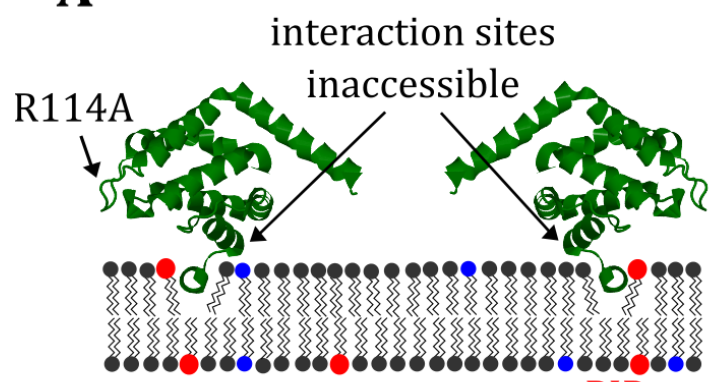

$\mathrm{PIP}_{2}$
B

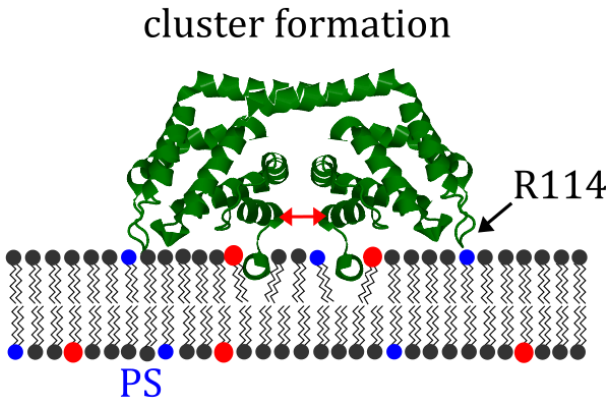

Figure 5.8: Orientation and interactions of two ENTH monomers in the (A) absence and (B) presence of PS. When PS is lacking less ENTH-membrane interactions occur. Due to the presence of PS interactions of ENTH with the membrane increase. Changed penetration depth and angles can lead to a facilitated interaction of $\mathrm{K} 23$ and $\mathrm{E} 42$ resulting in cluster formation.

Yoon et al. furthermore determined the insertion depth of the ENTH helix (usually $13 \AA^{2}$ from the phosphate group) as well as the penetration angle of the helix ( $11^{\circ}$ to the membrane normal) in the presence of PS. ${ }^{28}$ Lack of PS and/or R114 may change the orientation of the helix in the membrane which could lead to a different accessibility of the amino acids K23 and E42 for protein-protein interactions. Consequently less or no clusters were formed preventing imaging by AFM due to the mobile character of ENTH monomers (cf. chapter 5.1.2).

In summary, it can be stated that interactions of the amino acid R114 with the membrane or/and other ENTH monomers in the presence of PS are involved in the ENTH cluster formation.

\subsection{Lipid dependent penetration ability of ENTH to lipid monolay- ers}

Binding of ENTH to membranes illustrates the initial step of clathrin-mediated endocytosis (cf. chapter 1.1). As the ENTH-PIP2 binding leads to the formation of a newly formed $\alpha_{0}$ helix which can insert into the inner leaflet of the membrane, penetration experiments can help to understand the role of different lipids in this process. In the previous chapters, the importance of $\mathrm{PIP}_{2}$ and PS on the membrane recruitment and cluster formation was discussed. To analyze if these lipids also have an effect on the 
penetration ability of ENTH, the influence of PIP2 and PS on lipid packing densities and the protein surface activity will be discussed in more detail.

\subsubsection{Receptor lipid dependent penetration ability of ENTH}

In order to investigate the lipid packing of monolayers composed of POPC/POPE (80:20) and POPC/POPE/PIP2 (80-x:20:x, with $x=1,5,10$ mol\%), isotherms were recorded. The results show that the isotherms were shifted to smaller area per molecule values with increased $\mathrm{PIP}_{2}$ content. It has to be considered that the area per molecule values $\left(A_{\mathrm{M}}\right)$ was averaged over all lipids in the ratio of their molecular fraction. The monolayers of POPC/POPE lacking PIP 2 showed a similar compression behavior as the monolayer with 5 mol\% PIP2. Although the fatty acid chains of $\mathrm{PIP}_{2}$ molecules in sum exhibit lower degree of saturation than the fatty acid chains of POPC or POPE, ${ }^{44}$ different studies revealed similar area per molecule values for $\mathrm{PIP}_{2}$ and POPC at $30 \mathrm{mN} / \mathrm{m}$ (about $70 \AA^{2}$ ). ${ }^{172-175}$ Furthermore, it could be assumed that the negatively charged head group of $\mathrm{PIP}_{2}$ should increase the lipid packing due to electrostatic repulsion. ${ }^{173}$

However, despite the negative charges, PIP2 molecules can form hydrogen bonds which counteract the repulsion of the negative charge. ${ }^{176,177}$ This would explain that the area per molecule values of POPC and $\mathrm{PIP}_{2}$ are in the same range. Moreover, the increase in the PIP2 content could increase the amount of hydrogen bond, leading to a higher lipid packing and a shift of the isotherms to smaller area per molecule values. Besides the increased amount of hydrogen bonds, the shift in the surface pressurearea per molecule diagram can also be an effect of decreased amounts of lipids in the monolayer as they submerge into the subphase. If more $\mathrm{PIP}_{2}$ molecules submerge into the subphase, less molecules would be available for ENTH-PIP2 interactions within the monolayers. Hence the critical surface pressure and consequently the penetration ability could be underestimated.

In general, no collapse of monolayers composed of pure POPC, POPE or $\mathrm{PIP}_{2}$ until a surface pressure of $31 \mathrm{mN} / \mathrm{m}$ was observed in the literature. ${ }^{172-174,178,179}$ In this work, only the isotherms of POPC/POPE/PIP2 (79:20:1) monolayers showed irregularities 
or even collapsed between $27-31 \mathrm{mN} / \mathrm{m}$. This means that the critical surface pressure seems to be in this region and that further compression is not possible due to monolayer collapse. ${ }^{180}$ Furthermore, the area per molecule molecule per area $\left(A_{\mathrm{m}}\right)$ of the lipids in the POPC/POPE monolayers was slightly lower compared to the $A_{\mathrm{m}}$ in the 1 mol\% PIP2 monolayer which would support the assumption that a certain amount of $\mathrm{PIP}_{2}$ is necessary for the formation of stable hydrogen bonds. Thus, collapse appeared earlier even with a looser lipid packing compared to monolayers with 5 and 10 mol\% $\mathrm{PIP}_{2}$ (Figure 4.23).

It is only possible to measure the penetration ability of ENTH when the monolayer stability is ensured. Especially the experiments with $\mathrm{PIP}_{2}$ required the monolayer stability to guarantee that enough $\mathrm{PIP}_{2}$ molecules were accessible for interactions with the protein. Johnston et al. assumed the stability of different phospholipid monolayers when the decay rate did not exceed $1 \mathrm{mN} / \mathrm{m}$ per minute. ${ }^{115}$ Penetration experiments were only performed with POPC/POPE monolayers as monolayers composed of DOPC and DOPC/DOPE were not stable (data not shown). The decay rate in this work was smaller compared to Johnston et al. (decay rate $<1.5 \mathrm{mN} / \mathrm{m}$ per hour) for all used lipid mixtures. Although submerging of lipids into the subphase cannot be excluded completely, the monolayers were stable over $1 \mathrm{~h}$ once $30 \mathrm{mN} / \mathrm{m}$ was reached. This enabled the performance of monolayer penetration experiments. (cf. sections 3.3.5 and 4.3.1).

After addition of the protein, the surface pressure $(\pi)$ changed dependent on the initial value $\left(\pi_{0}\right)$. This increase is attributed to the insertion of the $\alpha_{0}$ helix. ${ }^{181}$ The change in surface pressure $(\Delta \pi)$ was inversely proportional to $\pi_{0}$, whereby the critical surface pressure $\left(\pi_{\mathrm{c}}\right)$ was determined as the $\mathrm{x}$-intercept. ${ }^{182,183}$ As the increments of $\pi$ often were irregular, no rate constants could be calculated. These irregularities were also found in penetration experiments with different proteins ${ }^{184,185}$ and were attributed to the uneven distribution of the ENTH in the subphase. In this setup, the mixing of the subphase after protein addition was only possible with a syringe, otherwise the monolayer was disturbed. Thus, distribution of the protein mainly took place by diffusion, which also increased the time to reach $\Delta \pi_{\text {max }}$.

Stahelin et al. e.g. used another Langmuir-Blodgett trough setup where a stirrer was used to achieve a homogenous distribution of the protein in the subphase. ${ }^{25}$ They 
monitored $\Delta \pi$ at a stirrer velocity of $60 \mathrm{rpm}$ reaching a maximum after $20 \mathrm{~min}$. In contrast to that, $\Delta \pi_{\max }$ here was reached after 10-120 min which could be explained by the different techniques to distribute the protein in the subphase. Previous experiments determined a critical surface pressure of $\pi_{c}=21 \mathrm{mN} / \mathrm{m}$ for a POPC/POPE (80:20) monolayer. 8,25 Also changes in the $\mathrm{pH}$ of the buffer did not influence the penetration ability essentially. In this thesis, ENTH also showed a poor penetration ability into POPC/POPE monolayers with $\pi_{\mathrm{c}}=23.3 \pm 0.8 \mathrm{mN} / \mathrm{m}$. As cell membranes and large unilamellar vesicles exhibit similar lipid packing densities compared to Langmuir-Blodgett monolayers at surface pressures in a range of $30-35 \mathrm{mN} / \mathrm{m}, 113,114,186$ the protein is only able to penetrate natural bilayer if $\pi_{c}$ exceeds $30 \mathrm{mN} / \mathrm{m}$. This in turn means that the ENTH domain is not able to penetrate POPC/POPE bilayers which is reasonable as the ENTH helix is only formed in the presence $\mathrm{PIP}_{2}{ }^{21}$

For monolayers with $\mathrm{PIP}_{2}, \pi_{\mathrm{c}}$ increased showing an influence of the receptor lipid on the surface activity of the protein. For monolayers with $1 \mathrm{~mol} \% \mathrm{PIP}_{2}, \pi_{\mathrm{c}}$ slightly increased $\left(\pi_{\mathrm{c}}=24.1 \pm 0.8 \mathrm{mN} / \mathrm{m}\right)$, but within the error of the measurements it was not significantly different. At $10 \mathrm{~mol} \% \mathrm{PIP}_{2}, \pi_{\mathrm{c}}$ changed to $28.7 \pm 0.6 \mathrm{mN} / \mathrm{m}$ and at $5 \mathrm{~mol} \%$ to $30.3 \pm 0.4 \mathrm{mN} / \mathrm{m}$. In both cases the $\pi_{\mathrm{c}}$ value was approximately around $30 \mathrm{mN} / \mathrm{m}$, indicating a high penetration capability into these monolayers. Hence, this means that ENTH is a membrane-active protein domain capable of penetrating the membrane when a certain amount of $\mathrm{PIP}_{2}$ is present.

$\pi_{\mathrm{c}}$ changed from $24.1 \pm 0.8 \mathrm{mN} / \mathrm{m}$ for $1 \mathrm{~mol} \% \mathrm{PIP}_{2}$ to values aroun $30 \mathrm{mN} / \mathrm{m}$ for 5 and $10 \mathrm{~mol} \% \mathrm{PIP}_{2}$ showing that the penetration ability of ENTH is a function of the $\mathrm{PIP}_{2}$ content. Furthermore, there is the possibility that for 1 mol\% $\mathrm{PIP}_{2}$ molecules submerge into the subphase during compression, resulting in less ENTH binding and penetration into the monolayer. In some cases, the isotherms of the monolayers with 1 mol\% PIP $_{2}$ showed a collapse at high surface pressures and this would support the assumption. A maximum of $\pi_{c}$ was observed when ENTH was added to monolayers containing 5 mol\% PIP $_{2}$. Since at higher PIP 2 concentrations more ENTH-PIP 2 interactions are expected due to previous results via RIfS (cf. chapter 4.1.3), the lipid packing could diminish a further insertion of the helix at $10 \mathrm{~mol} \% \mathrm{PIP}_{2}$ (Figure $5.9 \mathrm{~A}$ ). Moreover it is possible that $\mathrm{PIP}_{2}$ molecules submerged into the subphase or that $\mathrm{PIP}_{2}$ 
clusters could prevent the accessibility for ENTH binding (Figure 5.9 B and C). In all cases a reduced penetration of the ENTH helix would result.

A lipid packing

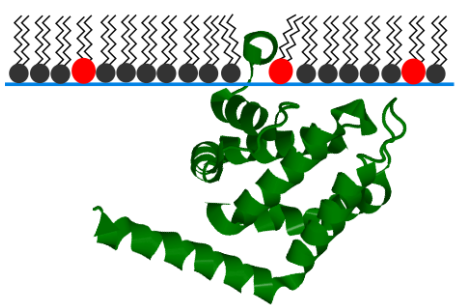

B

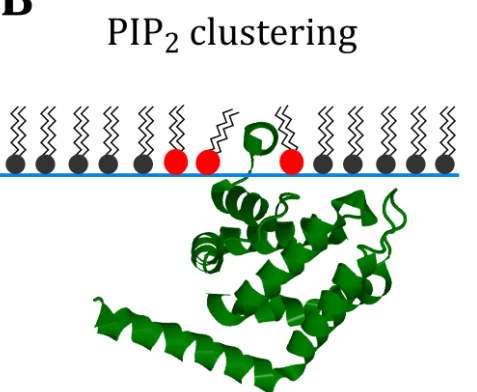

C $\mathrm{PIP}_{2}$ submerges into the subphase

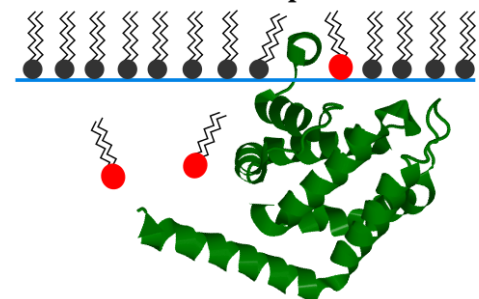

Figure 5.9: Schematic illustration of the ENTH penetration into monolayers composed of POPC/POPE/PIP2 (70:20:10). Three penetration mechanisms, which would explain the limited helix penetration into those monolayers, are conceivable: $(\mathrm{A})$ Insertion is prevented due to the dense lipid packing. (B) PIP2 clusters diminish the accessibility for ENTH binding. (C) PIP 2 molecules submerge into the subphase preventing the ENTH-PIP 2 binding within the monolayer.

Stahelin et al. and Hom et al. determined $\pi_{\mathrm{c}}$ values of $32 \mathrm{mN} / \mathrm{m}$ and $31 \mathrm{mN} / \mathrm{m}$ for POPC/POPE/PIP2 (77:20:3) and POPC/POPE/PIP2 (78:20:2) monolayers at pH 7.4.8,25 Hom et al. moreover showed that at $\mathrm{pH}$ of $6 \pi_{\mathrm{c}}$ decreased to $34.5 \mathrm{mN} / \mathrm{m}$ which was attributed to the facilitated ENTH binding to $\mathrm{PIP}_{2}$ due to protonation of histidine residues (H73 and H43) in the protein. The experiments in this work were performed at $\mathrm{pH} 7.4$ and therefore the measured $\pi_{\mathrm{c}}$ values are in good agreement with the literature, although the $\Delta \pi$ values at lower initial surface pressures were slightly smaller. Stahelin et al. used $3 \mathrm{~mol} \% \mathrm{PIP}_{2}$ and Hom et al. 2 mol\% PIP 2 instead of $5 \mathrm{~mol} \% \mathrm{PIP}_{2}$ which could explain the small differences. ${ }^{8,25}$ Moreover, even small changes in temperature and $\mathrm{pH}$ can lead to changes in the surface pressure due to the high sensitivity and also can affect the measurements. ${ }^{187}$

Although the experiments with POPC/POPE/PIP2 (79:20:1) monolayers showed a poor penetration ability of ENTH, RIfS measurements indeed showed specific binding of ENTH to supported POPC/PIP2 (99:1) monolayers with $\triangle O T=0.5 \pm 0.1 \mathrm{~nm}$ (cf. Figure 4.9). One possibility for this difference could be the stabilization of the monolayer by the used substrate and consequently a higher amount of accessible PIP2. On the other hand, the ENTH domain is recruited to POPC/POPE/PIP2 monolayers (and $\mathrm{POPC} / \mathrm{PIP}_{2}$ monolayer for RIfS), but only a minor amount of the proteins penetrated 
them. This would explain the low $\pi_{\mathrm{c}}$ of ENTH to 1 mol\% PIP2 monolayers. Furthermore, the protein penetration is only feasible up to an upper limit of the molecular packing. Many studies reported an optimum of the surface pressure in enzyme velocity and also the influence of the lipid packing density on protein penetration. ${ }^{188-191}$ Hence, this could support the assumption that the lipid packing in monolayers with 10 mol\% prevents further helix insertion.

In conclusion, the results show that $\mathrm{PIP}_{2}$ has a high impact on the penetration ability of ENTH as $\pi c$ significantly increases for monolayers.

\subsubsection{PS dependent penetration ability of ENTH}

Lipids with charged head groups like PS can have essential impact on different protein-membrane interactions. ${ }^{192,193}$ One example is the protein domain ENTH, whose binding affinity is increased in presence of PS (cf. chapter 4.2). Moreover, it was shown that PS induces the formation of ENTH clusters (Figure 4.17).

In order to investigate if PS can additionally affect the penetration ability of ENTH, Langmuir-Blodgett through measurements were performed. First, the compression behavior of monolayers in presence and absence of PS was analyzed. No differences in the isotherms were observed when the subphase was changed from water to HEPES buffer, even though the ionic strength can have an influence on the lipid packing. ${ }^{173,194}$ Furthermore no collapse of the monolayer was detected until the surface pressure of $32 \mathrm{mN} / \mathrm{m}$.

The monolayers lacking PS showed denser lipid packing (Figure 4.28) than the other monolayers with PS as they were shifted to lower area per molecule values.

Addition of PIP 2 to the PS monolayer even increased the area per molecule due to the incremented negative charges. These negative charges can repulse each other, ${ }^{195}$ which would increase the area per molecule. Although PIP 2 molecules can form intermolecular hydrogen bonds among themselves and counteract the negative repulsion ${ }^{173,174}$ the additional negative charges of PS seemed to diminish this counteraction. 
As all monolayers showed a high stability within one hour (percentage loss of $\pi<1.5 \mathrm{mN} / \mathrm{m}$ per hour), ${ }^{115}$ penetration experiments of the ENTH domain into those monolayers were performed and analyzed. Again, an irregular increase of $\pi$ after ENTH incubation was observed (cf. sections 4.3.1, 4.3.2 and 5.2.1) due to inhomogeneous protein distribution preventing the determination of rate constants. The increase of $\pi$ indicated the penetration of the ENTH helix. ${ }^{181}$ At high area per molecule values, the lipid packing density is low whereby insertion or surface activity of the protein can be measured, resulting in an inverse proportionality of $\Delta \pi$ to $\pi_{0}$. This behavior was also detected for the ENTH penetration experiment into PS-containing monolayers. The ENTH domain showed low penetration abilities into monolayers of POPC/POPE (80:20) with $\pi_{\mathrm{c}}=23 \mathrm{mN} / \mathrm{m}$ and POPC/POPE/POPS (60:20:20) with $\pi_{\mathrm{c}}=25 \mathrm{mN} / \mathrm{m}$ due to the lack of PIP2. Addition of PS slightly increased the penetration capability, especially at low area values. Consequently the negative charge of PS influ-

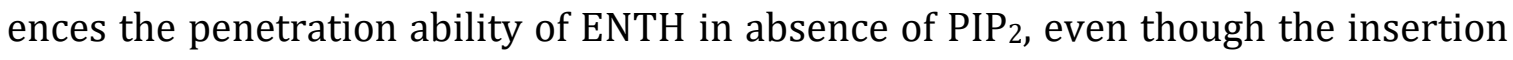
into cell membranes seems unlikely $\left(\pi_{\mathrm{c}}<30 \mathrm{mN} / \mathrm{m}\right) .{ }^{113,114,186}$ Compared to the POPC/POPE monolayers the POPC/POPE/POPS monolayers had a looser lipid packing (Figure 4.28) which would explain the slightly higher surface activity of the ENTH. RIfS experiments (Figure 4.13) showed that the negative charge of PS is not sufficient for ENTH-binding as the interaction is specific. ${ }^{24,164}$ Hence, the low penetration ability is reasonable.

The experiments with POPC/POPE/POPS/PIP2 (55:20:20:5) monolayers revealed slightly higher $\pi_{c}$ values with $32 \mathrm{mN} / \mathrm{m}$ compared to POPC/POPE/PIP 2 (75:20:5) monolayers with $\pi_{\mathrm{c}}=30 \mathrm{mN} / \mathrm{m}$. Within the error margin, the differences are negligible indicating that the penetration ability of ENTH into POPC/POPE/POPS/PIP2 monolayers is not significantly different compared to POPC/POPE/PIP 2 bilayers. In Figure $5.10, \pi_{\mathrm{c}}$ is plotted against the area per molecule at $\pi=20 \mathrm{mN} / \mathrm{m}$, showing that the lipid packing did not affected the ENTH helix penetration significantly. For a better overview, only values for 0 and $5 \mathrm{~mol} \% \mathrm{PIP}_{2}$ with and without PS were considered. The surface pressure of $20 \mathrm{mN} / \mathrm{m}$ was chosen to ensure that the monolayers did not collapse. 


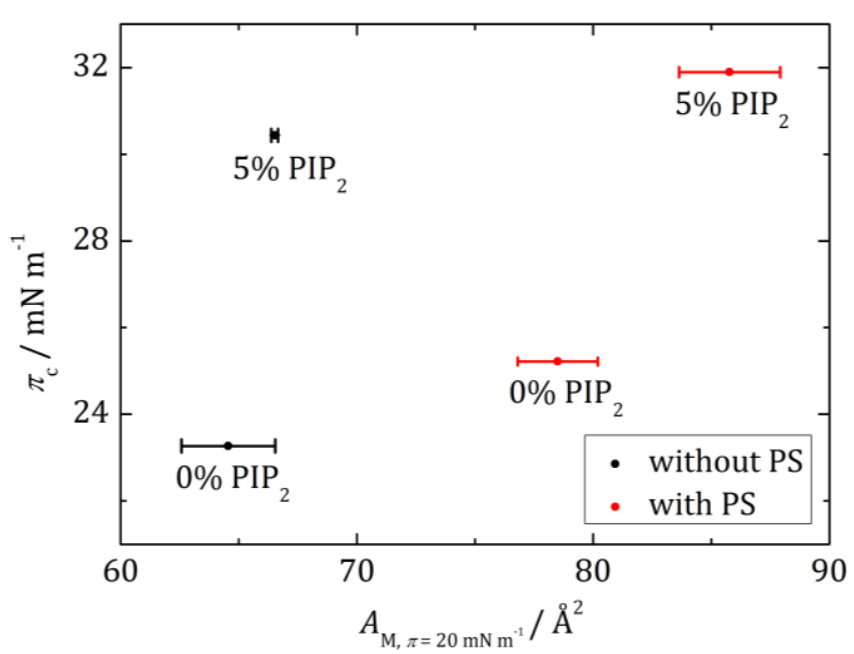

Figure 5.10: Illustration of $\pi_{\mathrm{c}}$ against the lipid packing at $\pi=20 \mathrm{mN} / \mathrm{m}$ for the lipid compositions POPC/POPE/PIP2 (80-x:20:x, with $\mathrm{x}=0$ or $5 \mathrm{~mol} \%$ ) and POPC/POPE/POPS/PIP2 (60-x:20:20:x, with $\mathrm{x}=0$ or $5 \mathrm{~mol} \%$ ) indicating that PS only slightly affects the penetration capability of ENTH although the lipid packing is decreased. Subphase: HEPES buffer.

The area per molecule increased about 15-20 $\AA^{2}$ when PS was present. Due to repulsive interactions, the area per molecule incremented resulting in a larger area for the lipids.

In different studies using vesicles, it was shown that the outer leaflet of the vesicles has a looser lipid packing at higher membrane curvatures. ${ }^{196-198}$ Moreover, the recruitment of hydrophobic helices to curved structures is preferred, ${ }^{153,199}$ thus penetration into a monolayer with a looser lipid packing was expected. Although the penetration is preferred when the lipid packing is low, the results show that the penetration is mainly a function of the $\mathrm{PIP}_{2}$ concentration at a $\mathrm{pH}$ of 7.4. Hom et al. revealed that the penetration capability of ENTH increased with decreased pH due to protonated histidine residues interacting with the monolayer. ${ }^{8}$ Therefore, changing the $\mathrm{pH}$ value might also increase penetration in case of PS. Furthermore the preferred helix insertion into membranes with looser lipid packing may facilitate penetration in curved structures, but not in flat Langmuir-Blodgett monolayers.

In contrast to this, binding studies by means of RIfS, PIP2 and PS containing bilayers showed a higher protein occupancy than the bilayers without PS (Figure 4.15). This indicates that ENTH adsorption to $\mathrm{PIP}_{2}$ doped membranes is facilitated, however the penetration ability is not affected. Hence, formation of clusters induced by PS could 
be a result of protein-protein interactions, where only a few ENTH monomers penetrate the monolayer (or the outer monolayer in supported bilayers) and the other monomers are interacting with the bound ENTH monomer without penetrating the membrane (Figure 5.11 A). As this cluster formation is not observed in the absence of PS (Figure 4.17), binding to PS could establish new binding sites for protein-protein interactions.

Yoon et al. assumed an orientation of two ENTH helices in an antiparallel manner and additionally interactions of the amino acids K23 und E42 in the presence of PS. ${ }^{28}$ Thus two binding structures of ENTH are conceivable. In Figure 5.11 the different feasible membrane-protein and protein-protein interactions in the presence of PS are illustrated.

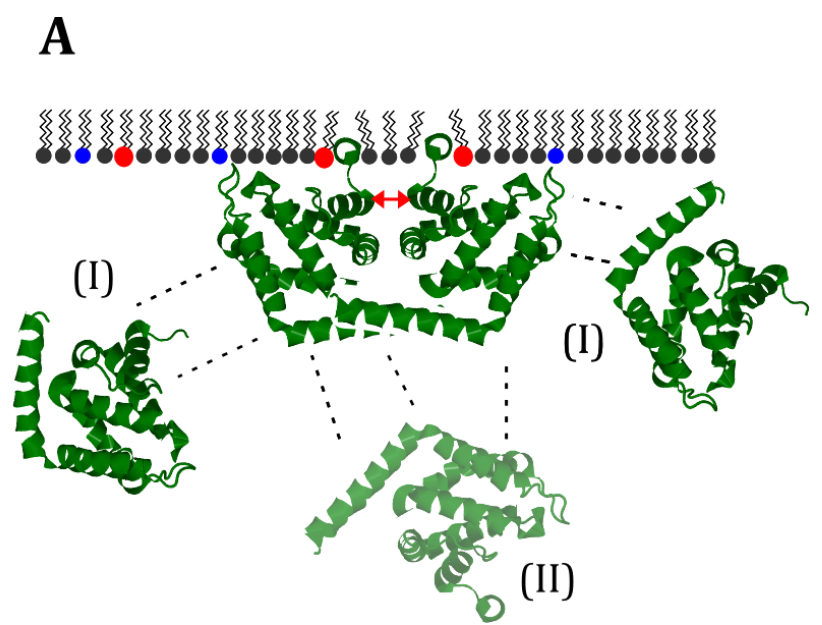

B

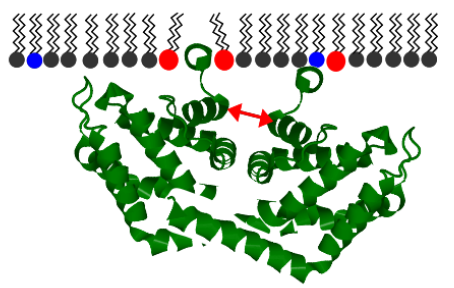

Figure 5.11: Schematic illustration of predicted ENTH-ENTH interactions resulting in protein clusters. Since penetration of ENTH into the monolayers in the presence of PS is not significantly increased although cluster formation was observed by means of AFM, interaction of the ENTH with PS could result in new interaction sites within the ENTH monomers for protein-protein interactions (A). In cases of (I) and (III) the result would be a higher protein occupancy on the surface. Case (II) seems less likely as the protein height observed via AFM showed lower monomer heights. (B) Furthermore, interactions of the helices in an antiparallel manner still can occur, when only one helix inserts into the monolayer. This would indicate a higher occupancy, but no further penetration.

The results so far show that the lipids $\mathrm{PIP}_{2}$ and PS have an impact on the ENTHmembrane interaction. Increase of $\mathrm{PIP}_{2}$ and addition of PS increased the amount of bound ENTH on bi- and monolayers. PIP2 specifically binds the ENTH and induces the formation of a new $\alpha_{0}$ helix which can insert into the membrane. PS additionally induced the formation of protein clusters. This in turn shows that the lipid composition 
contributes to the regulation of protein-dependent membrane deformation during CME.

\subsection{Adhered GUV as a membrane model to investigate protein- membrane interactions}

Adhesion of membranes and regulation of the membrane tension are substantial factors in biological processes, e.g. in exo- and endocytosis. ${ }^{200,201}$ In the previous chapters the impact of $\mathrm{PIP}_{2}$ and PS on the binding structure of ENTH in protein-membrane and protein-protein interactions was discussed in detail. In order to investigate if the ENTH can moreover affect the mechanical properties of membranes dependent on the lipid composition, adhered GUVs were utilized as membrane models.

The interactions of ENTH with $\mathrm{PIP}_{2}$ as a function of lateral membrane tension were already analyzed previously, ${ }^{79}$ thus in the next chapter the influence of ENTH on the membrane tension in the presence of PS was investigated.

\subsubsection{Adhesion of biotinylated GUVs on PEG-functionalized surfaces}

The formation of adhered GUVs requires a suitable surface to generate a contact area between the GUV and the substrate. Immobilization of GUVs via biotin-avidin interactions was already realized previously. Gleisner et al. incubated avidin on hydrophilized glass substrates. ${ }^{36}$ Addition of biotinylated GUVs composed of either DOPC/DOPE/cap-biotin-DOPE/PIP2/Atto488-DPPE (66:30:2:1:1) or DOPC/DOPE/ cap- biotin-DOPE/Atto488-DPPE (67:30:2:1) resulted in immobile vesicles with a contact area to the surface. The lateral membrane tensions of these adhered GUVs ranged from 0.01 to $1.02 \mathrm{mN} / \mathrm{m} .{ }^{36}$ Regulation of the membrane tension was realized by varying the concentration of $\mathrm{MgCl}_{2}$ in the surrounding buffer and was attributed to stronger interactions of $\mathrm{PIP}_{2}$ and the $\mathrm{Mg}^{2+}$ ions. Thereby, smaller membrane tensions were determined for GUVs lacking PIP2 compared to GUVs with PIP2. 
In this thesis, the impact of PS on the membrane tension of GUVs was investigated using similar conditions as Gleisner et al. ${ }^{36}$ Since negative charges of PS and PIP2 are known to cluster in the presence of $\mathrm{MgCl}_{2}$, regulation of the membrane tension with the $\mathrm{MgCl}_{2}$ concentration was excluded.125,126 Moreover, it was shown that NeutrAvidin exhibits less non-specific interactions than avidin in immunoassays while maintaining a high binding affinity to biotin $\left(\sim 1.3 \cdot 10^{-9} \mu \mathrm{M}\right) .{ }^{127,202}$ Thus, it is assumed to be promising for biosensing applications. To reduce unspecific interactions of the negative charges of PS/PIP 2 and avidin to a minimum, avidin was replaced by the deglycosylated protein NeutrAvidin.

However, incubation of NeutrAvidin on hydrophilized glass substrates resulted in an inhomogenous distribution of the protein on the surface (Figure 4.31) and immobilization of the vesicles via NeutrAvidin-biotin binding was not possible as the protein was removed from the surface during rinsing. Avidin has its isoelectric point at $\mathrm{pH}$ 10.5 and it is positively charged at a $\mathrm{pH}$ of 7.4. ${ }^{203}$ This promotes the electrostatic interactions of avidin with the surface. Compared to that, NeutrAvidin exhibits an isoeletric point of 6.3, which leads to less non-specific interaction with the substrate surface, while the high binding affinity to biotin is still existing ${ }^{60,127}$ and would explain the inhomogenous coverage of NeutrAvidin on the hydrophilized glass surface. On this account, the strategy for NeutrAvidin immobilization had to be changed. A method to immobilize vesicles bases on the work of Piehler et al. was used. ${ }^{129}$ Silanization of glass substrates with (3-Glycidyloxypropyl)trimethoxysilane (GOPTS) resulted in a surface with epoxide moieties. Depending on the added reagent, the functionalization on the substrate surface was regulated (Figure 3.3). J. Thiart and Schwamborn et al. utilized this technique and coupled amino-terminated Poly(ethylen glycol) (PEG) groups to the surface. ${ }^{128,204}$ This solvent-free coupling with amino groups of bifunctional PEGs enabled immobilization of vesicles on these surfaces. Using biotin-PEG-NH 2 (b-PEG) ensured the affinity for NeutrAvidin and methoxy-PEG$\mathrm{NH}_{2}$ (m-PEG) was used to obtain a lateral distance between the biotin groups to be accessible for binding (Figure 5.12). 


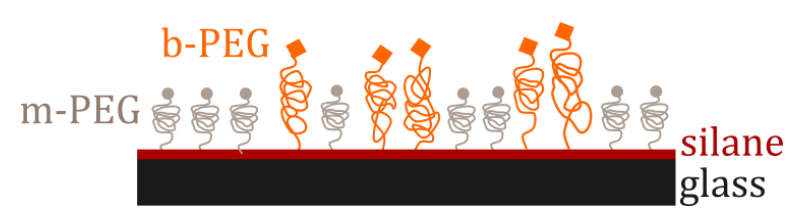

Figure 5.12: PEG-based surface functionalization. After silanization and PEGylation of glass substrates, the biotin-PEG (b-PEG)/methoxy-PEG (m-PEG) covered surface was incubated with NeutrAvidin, enabling the immobilization of biotinylated GUVs on these surfaces.

In contrast to the studies of J.Thiart and Schwamborn et al., b-PEG and m-PEG species with less PEG groups were used $(M(\mathrm{~m}-\mathrm{PEG})=350 \mathrm{~g} / \mathrm{mol}$ and $M(\mathrm{~b}-\mathrm{PEG})=600 \mathrm{~g} / \mathrm{mol}$ instead of $M(\mathrm{~m}-\mathrm{PEG})=2000 \mathrm{~g} / \mathrm{mol}$ and $M(\mathrm{~b}-\mathrm{PEG})=3000 \mathrm{~g} / \mathrm{mol})$. PEG is known to reduce the adhesion of proteins or other molecules and is used to protect surfaces. ${ }^{205,206}$ Thereby, the PEG density on the surface and the chain length can influence the adsorption of molecules. ${ }^{207}$ Since an ordered structure of PEGs on the surface cannot be guaranteed, biotin molecules could be less accessible. Therefore, the amount of PEG groups was reduced to diminish the repelling character of the PEG surface.

Incubation of these m-PEG/b-PEG surfaces with labeled NeutrAvidin yielded an even distribution in the fluorescence intensity of the labeled protein on the surface (Figure 4.31) indicating a homogenous coverage of NeutrAvidin on the substrates. As the bPEGs were covalently bound to the silane on the glass surface, the NeutrAvidin also was immobilized due to the high affinity to biotin and was not removed by rinsing with buffer.

Since a homogenous NeutrAvidin occupancy on the PEG surfaces was realized, the system was assumed to be suitable to immobilize biotinylated GUVs via further NeutrAvidin-biotin interactions. Therefore, GUVs composed of DOPC/DOPS/Cholesterol/ cap-biotin-DOPE/PIP2/Atto488-DPPE (76:10:10:2:1:1) were added to the system resulting in adhered GUVs on the b-PEG-NeutrAvidin surface(Figure 5.13). 


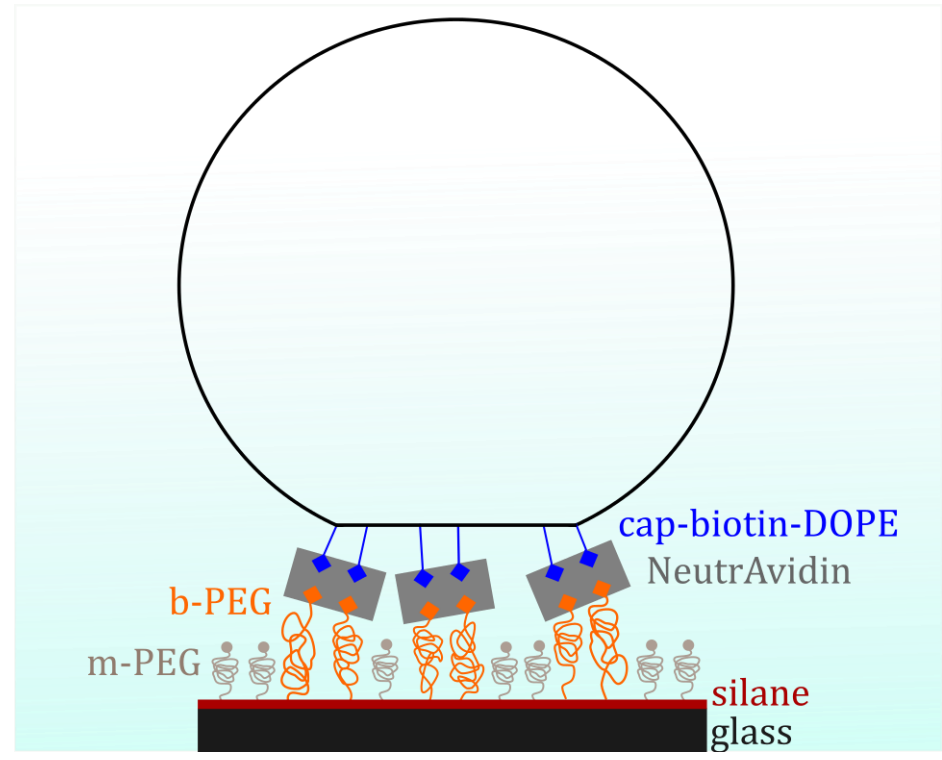

Figure 5.13: Schematic illustration of the PEG-based surface functionalization. After silanization and PEGylation of glass substrates, the biotin-PEG (b-PEG)/methoxy-PEG (m-PEG) covered surface was incubated with NeutrAvidin enabling the immobilization of biotinylated GUVs on these surfaces.

These GUVs were stable over $2 \mathrm{~h}$, which was proved by determination of the $r_{\mathrm{i}} / r_{\mathrm{ad}}$ values of the adhered GUVs directly after immobilization (15 min) and after $2 \mathrm{~h}$ (Figure 4.33). The $r_{\mathrm{i}} / r_{\text {ad }}$ values did not significantly change within the deviation (from $0.16 \pm 0.04$ to $0.17 \pm 0.04$ ) indicating that the GUVs were stable over time. Most likely the stability was maintained due to iso-osmolar conditions using a humidity chamber. The membrane tensions of these GUVs were measured to be $(1.03 \pm 0.04) \cdot 10^{-2} \mathrm{mN} / \mathrm{m}$ (after $15 \mathrm{~min}$ ) and $(1.03 \pm 0.03) \cdot 10^{-2} \mathrm{mN} / \mathrm{m}$ (after $2 \mathrm{~h}$ ). The values in this thesis are comparable to cellular tensions which are in the range of $0.002-0.3 \mathrm{mN} / \mathrm{m}$ depending on the cell type. ${ }^{201,208-210}$ Compared to the membrane tensions of GUVs adhered to avidin coated glass surfaces measured by Gleisner et al., the values of this work lay in the low membrane tension range.

To mimic membrane tensions of different cell types, the lateral membrane tension of the adhered GUVs should be regulated. Due to this, the amount of biotin on the surface was varied by changing the ratio of m-PEG and b-PEG to see if the amount of accessible biotin molecules could be increased. NeutrAvidin has 4 binding sites for biotin, 2 on each side (cf. Figure 5.13),58 thus a higher concentration of biotin on the surface could increase the coverage of NeutrAvidin on the surface. However the membrane tension of the GUVs was not affected by variations in the m-PEG/b-PEG ratio (Figure 
4.34). Since NeutrAvidin has a high binding affinity to biotin, the surface could be completely covered at a ratio of 1:1.127,202 Thus further raise of the b-PEG concentration would not affect the protein occupancy and the adhesion of biotynilated GUVs.

Another possibility to regulate the membrane tension could be achieved by changing the lipid composition. Nevertheless, it was not tried to vary the membrane tension as a function of the lipid composition because the search for an adequate lipid composition, resulting in stable GUVs, was challenging. However, the increase of biotin-coupled lipids may facilitate binding to the NeutrAvidin surfaces and consequently increase the membrane tension.

Moreover, the variation of the negative charges ( $\mathrm{PIP}_{2}$ and PS) in the lipids could have an impact on the membrane tensions. Raucher et al. already showed with experiments on NIH-3T3 cells that PIP2 can control the adhesion. ${ }^{211}$ Raise of the PS concentrations in combination with this surface functionalization though, was not possible as these vesicles were unstable or too small to determine the membrane tension based on the geometry. Although GUVs with higher proportions of negative charges $(\sim 25 \%)$ were already generated,212,213 incorporation of negative charges is limited due to repulsive interactions and cluster formations. ${ }^{214,215}$ As higher PS concentrations were already realized in free vesicles, it is more likely that the PEG functionalization and the adhesion (higher membrane tensions) also had an impact on the GUV stability when a higher PS content was incorporated in the vesicles.

Thus, before the lipid composition was further adjusted, the impact of ENTH on the membrane tension of GUVs composed of DOPC/DOPS/Cholesterol/cap-biotinDOPE/PIP2/Atto488-DPPE (76:10:10:2:1:1) was investigated in detail.

\subsubsection{Effect of the ENTH binding to PS-containing adhered GUVs}

Once stable adhered GUVs were generated and the membrane tension was determined, the ENTH binding activity on these membranes was analyzed. After incubation of GUVs composed of DOPC/DOPS/Cholesterol/cap-biotin-DOPE/PIP2/Atto488DPPE (76:10:10:2:1:1) with $1 \mu \mathrm{M}$ ENTH for $2 \mathrm{~h}$, the solution contained less GUVs and 
a lot of lipid material outside the vesicles was observed. The amount of vesicles before and after ENTH addition was not counted, but the difference was obvious. During imaging of the remaining GUVs, the vesicles ruptured. This only happened when ENTH was present (Figure 4.37). In the absence of ENTH, no rupturing event occurred within $2 \mathrm{~h}$ (Figure 4.33) indicating that the rupture of the vesicles is affected by the protein adsorption to the PIP2 doped GUVs. Reduction of the ENTH (0.3 $\mu \mathrm{M})$ concentration still resulted in GUV rupturing (Figure 4.38) showing that even significantly lower concentrations are sufficient to induce GUV bursting.

In clathrin-mediated endocytosis (CME), binding of the ENTH domain to $\mathrm{PIP}_{2}$ results in the formation of a new amphipathic helix which inserts into the inner leaflet of the membrane. ${ }^{21}$ The insertion of the helix disturbs the membrane and consequently can influence the lipid packing in the membrane. ${ }^{7}$ In this work an analogous behavior for the adhered GUVs might cause an insertion into the outer leaflet.

Therefore, different reasons for the presence of less GUVs and the vesicle rupture are conceivable. First of all, the helix insertion can cause membrane defects due to the disturbance of the lipid packing. 216,217 This could destabilize the vesicles, leading to the breakdown of the osmolarity gradient and consequently rupture of the vesicles. Different studies revealed that insertion of an amphipathic helix is preferred to small hydrophobic lipid bilayer packing defects. ${ }^{218-220}$ This is also consistent with the fact that ENTH favors binding to curved membranes as they exhibit more packing defects. ${ }^{221}$ Preferred insertion of the ENTH helix into those membranes can facilitate membrane deformation or induction of membrane defects. Although the bilayer structure of GUVs seem to be flat in the lipid scale, they exhibit a higher curvature compared to flat Langmuir-Blodgett monolayers. Thus, a slightly higher insertion rate of the ENTH helix can be assumed. In the adhered GUVs the PS could additionally increase the insertion of the ENTH helix, even though it was not shown in LangmuirBlodgett monolayers.

Furthermore, the helix insertion reduces the bending rigidity of the membrane. A reduced bending stiffness lowers the energy which is required for membrane deformation. ${ }^{79}$ Due to this, the formation of tubules or even vesicles out of the GUV is facilitated (Figure 5.14) which leads to the destabilization of the vesicles. 


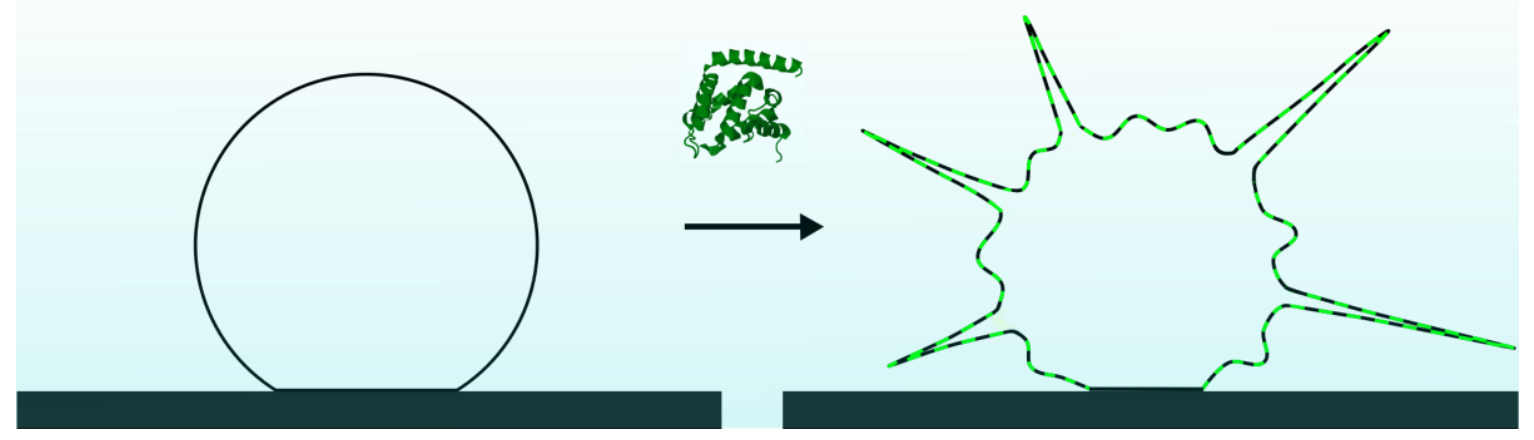

Figure 5.14: Binding of ENTH (green) to adhered GUVs resulting in the formation of tubules, which furthermore destabilizes the adhered GUV.

During the formation of these structures the size of the vesicle decreases until all lipid material is dissipated. This would explain why usually less GUVs were found in the solution after ENTH addition. Tubulation of vesicles due to ENTH adsorption was already reported in the literature. Yoon et al. showed that incubation of free vesicles composed of POPC/POPE/POPS/PIP2/Rh-PE (46.5:30:20:3:0.5) with 0.5 $\mu \mathrm{M}$ ENTH resulted in the appearance of tubular structures. ${ }^{28}$ Gleisner et al. showed with studies on adhered GUVs that at low membrane tensions $(0.08 \mathrm{mN} / \mathrm{m})$ tubular structures were observed after the ENTH addition $(1 \mu \mathrm{M})$. In contrast to that adhered GUVs flattened at high membrane tensions when ENTH was present. The adhered GUVs in this thesis exhibited even lower membrane tensions $(0.01 \mathrm{mN} / \mathrm{m})$, indicating that tubulation seems likely.

However, some studies revealed that tubulation only occurs, when the protein coverage of the surface is above 20\%. ${ }^{30}$ At high ENTH concentrations and 1 mol\% PIP 2 concentration, a protein coverage of maximum $23 \%$ should be reached considering the footprint of ENTH $\left(16 \mathrm{~nm}^{2}\right)^{22}$ and the surface demand of the receptor lipid $\left(0.7 \mathrm{~nm}^{2}\right) .{ }^{44}$ At a concentration of $1 \mu \mathrm{M}$ this decreases to $16 \%$ when the $K_{\mathrm{D}}$ of $0.42 \mu \mathrm{M}$ is noted, which should not induce tubulations. Nevertheless, tubulation was already observed at lower protein coverage (11.4\%) even in the absence of PS79

Addition of PS might facilitate the tubulation. It was shown that PS has a significant impact on the binding as a higher binding affinity and a higher protein occupancy on the bilayer surface were measured in the presence of PS (cf. chapter 4.2.2). This was 
attributed to the cluster formation induced by PS. Protein crowding was shown to destabilize membrane shapes. ${ }^{35}$ Cluster formation and the insertion of the ENTH helix in one region of the GUVs would lead to instabilities, hence the rupturing seems reasonable.

Furthermore, heterogeneities of the lipid distribution within the GUVs could lead to a higher amount of $\mathrm{PIP}_{2}$ and PS in some vesicles. Different studies revealed that the heterogeneities of the lipid compositions in one GUV preparation via electroformation can vary from 2 to $13 \% .^{222,223}$ If more PIP 2 and PS are present in some GUVs compared to others (excluding $\mathrm{PIP}_{2}$ clustering), this would lead to a higher amount of bound ENTH and would explain the reduced amount of GUVs as they burst earlier due to defects caused by the helix insertion of ENTH.

Krishnan et al. showed that the interactions of $\mathrm{PIP}_{2}$ with the actin-binding protein profilin led to the destabilization of GUVs. ${ }^{224}$ From 8-10 mol\% $\mathrm{PIP}_{2}$ they recognized the start of $\mathrm{PIP}_{2}$ cluster formation. Further increase of the concentration destabilized the GUVs due to the local membrane deformation by the clusters. If some vesicles exhibit high local accumulations of $\mathrm{PIP}_{2}$, it can lead to destabilization.

Considering that some GUVs were imaged in the first seconds and only ruptured during the time-series, perhaps also the laser irradiation had an influence on the GUV stability. Brückner et al. induced changes in the GUV shape with UV light. ${ }^{225}$ Other experiments varied the area/volume ratio by temperature dependent vesicle shape changes. ${ }^{226}$ Although the energy of UV (10-400 nm) and visible light (here $\sim 600 \mathrm{~nm}$ ) differ and the temperature changes by the laser should be negligible, an impact of the laser illumination cannot be excluded as GUVs rupture during imaging.

As the amount of GUVs during incubation with ENTH seemed to decrease, a combination of different effects is assumed as its cause. First of all, the helix insertion disturbs the bilayer and leads to lipid packing defects which destabilize the GUV. Secondly, the presence of PS induces protein crowding, facilitating membrane deformation and increasing membrane defects. Finally, the laser illumination leads to the rupture of the remaining destabilized vesicles.

In conclusion, it was shown successfully that the analysis of adhered GUVs on b-PEGNeutrAvidin functionalized surfaces is possible. However, the system is only suitable to a limited extent to investigate the impact of ENTH on the mechanical properties of 
these adhered GUVs in the presence of PS. Reducing the PS concentration or/and repelling character of the PEG surface (e.g. further decrease of the amount of PEG groups) might improve the system. 



\section{ConClusion}

Membrane remodeling processes which occur during endocytosis, are regulated by the complex interactions between proteins and lipid membranes. The most important pathway for endocytosis is the clathrin-mediated endocytosis (CME), where adaptor proteins like epsin induce a membrane invagination by specific binding to the receptor lipid phosphatidylinositol-(4,5)-bisphosphate (PIP2). In this thesis the lipid dependent binding of the epsin $N$-terminal homology (ENTH) domain to artificial membrane models was analyzed.

The binding studies of ENTH to supported lipid bilayer and monolayers doped with $\mathrm{PIP}_{2}$ revealed an asymmetric distribution of the specific receptor lipid within both leaflets of a bilayer. Increasing the $\mathrm{PIP}_{2}$ concentration resulted in an increased optical thickness and consequently a higher occupancy on the membrane surface. Only in case of monolayers nearly a complete coverage was achieved with an optical thickness of $2.2 \pm 0.4 \mathrm{~nm}$.

Addition of lipids with the negatively charged head group phosphatidylserine (PS) increased the binding affinity of ENTH to PIP2 containing membranes. Since the ENTH did not bind to POPC/POPS bilayers in the absence of $\mathrm{PIP}_{2}$, the specific binding to $\mathrm{PIP}_{2}$ and not to the negative charge by non-specific electrostatic interactions was proved. The dissociation constant of ENTH binding to a POPC/POPS/PIP 2 bilayer was about twice as high as to a POPC/PIP 2 bilayer. Moreover, the maximum in optical thickness upon protein binding increased from $1.6 \pm 0.1 \mathrm{~nm}$ (without PS) to $2.5 \pm 0.1 \mathrm{~nm}$ (with PS) at the same PIP2 concentration, showing a relevant effect of PS on the binding behavior of ENTH to those membranes. Analysis of the surface topography before and after ENTH adsorption indicated that this increased occupancy resulted from cluster formation of ENTH monomers when PS was present. In the absence of PS no clusters or single molecules were detected on the bilayer surface due to the high mobility of the single ENTH monomers.

Monolayer penetration experiments were performed to investigate the penetration ability of ENTH into monolayers as a function of the surface pressure dependending on the lipid composition. The critical surface pressure is the maximum value, where 
no insertion of proteins into the monolayer is possible anymore. Increasing the $\mathrm{PIP}_{2}$ content first increased the penetration capability and reached a maximum at $5 \mathrm{~mol} \%$ $\mathrm{PIP}_{2}$ with $\pi_{\mathrm{c}}=30.3 \pm 0.4 \mathrm{mN} / \mathrm{m}$.

The lipid packing was then changed by the addition of PS, only slightly increasing the critical surface pressure to $\pi_{\mathrm{c}}=32.0 \pm 0.9 \mathrm{mN} / \mathrm{m}$, although a higher binding affinity and protein coverage on $\mathrm{PIP}_{2} / \mathrm{PS}$ doped membranes was observed. This indicates that PS is inducing protein clusters, which only partly penetrate the membrane.

Adhered GUVs as membrane models enabled to measure ENTH activity as a function of membrane tension. The membrane tension of GUVs adhered to NeutrAvidin functionalized substrates was determined to be $0.01 \mathrm{mN} / \mathrm{m}$. After incubation with ENTH, these GUVs ruptured induced by the insertion of the ENTH helix and consequently appearance of lipid packing defects in the membrane.

In conclusion, it was shown that ENTH binding to membranes is highly regulated by the lipid composition. Furthermore, the results revealed that the membrane deformation in CME induced by ENTH is an interplay of the ENTH helix insertion and the crowding mechanism induced by $\mathrm{PIP}_{2}$ and PS. 


\section{BibliOgRAPHY}

1. McMahon, H. T. \& Boucrot, E. Molecular mechanism and physiological functions of clathrin-mediated endocytosis. Nat. Rev. Mol. Cell Biol. 12, 517-533 (2011).

2. Conner, S. D. \& Schmid, S. L. Regulated portals of entry into the cell. Nature $\mathbf{4 2 2}$, 37-44 (2003).

3. Sandvig, K. \& Van Deurs, B. Endocytosis, intracellular transport, and cytotoxic action of Shiga toxin and ricin. Physiol. Rev. 76, 949-966 (1996).

4. Banerjee, A., Berezhkovskii, A. \& Nossal, R. Kinetics of cellular uptake of viruses and nanoparticles via clathrin-mediated endocytosis. Phys. Biol. 13, 016005 (2016).

5. Hurley, J. H. \& Wendland, B. Endocytosis: driving membranes around the bend. Cell 111, 143-146 (2002).

6. Wendland, B., Steece, K. E. \& Emr, S. C. Yeast epsins contain an essential N-terminal ENTH domain, bind clathrin and are required for endocytosis. EMBO J. 18, 43834393 (1999).

7. Horvath, C. A. J., Vanden Broeck, D., Boulet, G. A. V., Bogers, J. \& De Wolf, M. J. S. Epsin: Inducing membrane curvature. Int. J. Biochem. Cell Biol. 39, 1765-1770 (2007).

8. Hom, R. A., Vora, M., Regner, M., Subach, O. M., Cho, W., Verkhusha V. V., Stahelin, R. V. \& Kutateladze T. G. pH-dependent Binding of the Epsin ENTH Domain and the AP180 ANTH Domain to PI(4,5)P2-containing Bilayers. J. Mol. Biol. 373, 412-423 (2007). 
9. Meinecke, M., Boucrot, E., Camdere, G., Hon, W-C. Mittal, R. \& McMahon H. T. Cooperative Recruitment of Dynamin and BIN/Amphiphysin/Rvs (BAR) Domain-containing Proteins Leads to GTP-dependent Membrane Scission. J. Biol. Chem. 288, 6651-6661 (2013).

10. Kaksonen, M. \& Roux, A. Mechanisms of clathrin-mediated endocytosis. Nat. Rev. Mol. Cell Biol. 19, 313-326 (2018).

11. Messa, M., Fernández-Busnadiego, R., Sun, E. W., Chen, H., Czapla, H., Wrasman, K., Wu, Y., Ko, G., Ross, T., Wendland, B. \& De Camilli, P. Epsin deficiency impairs endocytosis by stalling the actin-dependent invagination of endocytic clathrincoated pits. eLife 3, e03311 (2014).

12. Ungewickell, E. J. \& Hinrichsen, L. Endocytosis: clathrin-mediated membrane budding. Curr. Opin. Cell Biol. 19, 417-425 (2007).

13. De Camilli, P., Chen, H., Hyman, J., Panepucci, E., Bateman, A. \& Brunger A. T. The ENTH domain. FEBS Lett. 513, 11-18 (2002).

14. Schmidt, A. A. The making of a vesicle. Nature 419, 347-349 (2002).

15. Legendre-Guillemin, V., Wasiak, S., Hussain, N. K., Angers, A. \& McPherson P. S. ENTH/ANTH proteins and clathrin-mediated membrane budding. J. Cell Sci. 117, 9-18 (2004).

16. Slepnev, V. I. \& De Camilli, P. Accessory factors in clathrin-dependent synaptic vesicle endocytosis. Nat. Rev. Neurosci. 1, 161-172 (2000).

17. Nossal, R. \& Zimmerberg, J. Endocytosis: Curvature to the ENTH Degree. Curr. Biol. 12, R770-R772 (2002). 
18. Chen, H., Fre, S., Slepnev, V. I., Capua, M. R., Takei, K., Butler, M. H., Di Fiore, P. P. \& De Camilli P. Epsin is an EH-domain-binding protein implicated in clathrinmediated endocytosis. Nature 394, 793-797 (1998).

19. Polo, S., Sigismund, S., Faretta, M., Guidi, M., Capua, M. R., Bossi, G., Chen, H., De Camilli, P. \& Di Fiore, P. P. A single motif responsible for ubiquitin recognition and monoubiquitination in endocytic proteins. Nature 416, 451-455 (2002).

20. Sen, A., Madhivanan, K., Mukherjee, D. \& Aguilar, R. C. The epsin protein family: coordinators of endocytosis and signaling. Biomol. Concepts 3, (2012).

21. Ford, M. G. J., Mills, I. G., Peter, B. J., Vallis, Y., Praefcke, G. J. K., Evans, P. R. \& McMahon H. T. Curvature of clathrin-coated pits driven by epsin. Nature 419, 361366 (2002).

22. Ford, M. G. J., McMahon, H. T. \& Evans, P. R. Epsin ENTH bound to Ins(1,4,5)P3. (2000) doi:10.2210/pdb1H0A/pdb.

23. Fremont, D. Crystal structure of the epsin N-terminal homology domain (ENTH) at 1.56 Angstrom resolution. (2000) doi:10.2210/pdb1EYH/pdb.

24. Itoh, T., Koshiba, S., Kigawa, T., Kikuchi, A., Yokoyama, S. \& Takenawa, T. Role of the ENTH Domain in Phosphatidylinositol-4,5-Bisphosphate Binding and Endocytosis. Science 291, 1047-1051 (2001).

25. Stahelin, R. V., Long, F., Peter, B. J., Murray, D., De Camilli, P., McMahon H. T. \& Cho, W. Contrasting Membrane Interaction Mechanisms of AP180 N-terminal Homology (ANTH) and Epsin N-terminal Homology (ENTH) Domains. J. Biol. Chem. 278, 28993-28999 (2003).

26. Kroppen, B. Molecular details of membrane deformation by ENTH domains. Dissertation, University of Göttingen (2017). 
27. Itoh, T. \& Decamilli, P. BAR, F-BAR (EFC) and ENTH/ANTH domains in the regulation of membrane-cytosol interfaces and membrane curvature. Biochim. Biophys. Acta Mol. Cell Biol. Lipids 1761, 897-912 (2006).

28. Yoon, Y., Tong, J., Lee, P. J., Albanese, A., Bhardwaj, N., Källberg, M., Digman, M. A., Lu, H., Gratton, E., Shin, Y-K. \& Cho, W. Molecular Basis of the Potent Membraneremodeling Activity of the Epsin 1 N-terminal Homology Domain. J. Biol. Chem. 285, $531-540$ (2010).

29. Ford, M. G. J., Pearse, M. F., Higgins, M. K., Vallis, Y., Owen, D. J., Gibson, A., Hopkins, C. R., Evans, P. R. \& McMahon H. T. Simultaneous Binding of PtdIns(4,5)P2 and Clathrin by AP180 in the Nucleation of Clathrin Lattices on Membranes. Science 291, 1051-1055 (2001).

30. Stachowiak, J. C., Schmid, E. M., Ryan, C. J., Ann, H. S., Sasaki, D. Y., Sherman, M. B., Geissler, P. L., Fletcher, D. A \& Hayden C. C. Membrane bending by protein-protein crowding. Nat. Cell Biol. 14, 944-949 (2012).

31. Campelo, F., McMahon, H. T. \& Kozlov, M. M. The Hydrophobic Insertion Mechanism of Membrane Curvature Generation by Proteins. Biophys. J. 95, 2325-2339 (2008).

32. Blood, P. D., Swenson, R. D. \& Voth, G. A. Factors Influencing Local Membrane Curvature Induction by N-BAR Domains as Revealed by Molecular Dynamics Simulations. Biophys. J. 95, 1866-1876 (2008).

33. Gallop, J. L. Jao, C. C., Kent, H. M., Butler, P. J. G., Evans, P. R., Langen, R. \& McMahon H. T. Mechanism of endophilin N-BAR domain-mediated membrane curvature. EMBO J. 25, 2898-2910 (2006). 
34. Kozlov, M. M., Campelo, F., Liska, N., Chernomordik, L. V., Marrink, S. J. \& McMahon H. T. Mechanisms shaping cell membranes. Curr. Opin. Cell Biol. 29, 5360 (2014).

35. Chen, Z., Atefi, E. \& Baumgart, T. Membrane Shape Instability Induced by Protein Crowding. Biophys. J. 111, 1823-1826 (2016).

36. Gleisner, M., Kroppen, B., Fricke, C., Teske, N., Kliesch, T-T., Janshoff A., Meinecke, M. \& Steinem, C. Epsin N-terminal Homology Domain (ENTH) Activity as a Function of Membrane Tension. J. Biol. Chem. 291, 19953-19961 (2016).

37. van Meer, G., Voelker, D. R. \& Feigenson, G. W. Membrane lipids: where they are and how they behave. Nat. Rev. Mol. Cell Biol. 9, 112-124 (2008).

38. Segev, N. Trafficking Inside Cells: Pathways, Mechanisms and Regulation. Springer-Verlag, New York (2009), 210-227.

39. Harwood, J. L. et al. Lipids: biology and health. (John Wiley \& Sons Inc, 2016), $6^{\text {th }}$ Edition

40. Pinot, M., Vanni, S., Pagnotta, S., Lacas-Gervais, S., Payet, L-A., Ferreira, T., Gautier, R., Goud, B., Antonny, B. \& Barelli, H. Polyunsaturated phospholipids facilitate membrane deformation and fission by endocytic proteins. Science 345, 693-697 (2014).

41. Kirchhausen, T. Bending membranes. Nat. Cell Biol. 14, 906-908 (2012).

42. Devaux, P. F. Lipid transmembrane asymmetry and flip-flop in biological membranes and in lipid bilayers. Curr. Opin. Struct. Biol. 3, 489-494 (1993).

43. Devaux, P. F. Static and dynamic lipid asymmetry in cell membranes. Biochemistry 30, 1163-1173 (1991). 
44. McLaughlin, S., Wang, J., Gambhir, A. \& Murray, D. PIP2 and Proteins: Interactions, Organization, and Information Flow. Annu. Rev. Biophys. Biomol. Struct. 31, 151-175 (2002).

45. Roldan, E. R. S. \& Harrison, R. A. P. Polyphosphoinositide breakdown and subsequent exocytosis in the $\mathrm{Ca}^{2+}$ /ionophore-induced acrosome reaction of mammalian spermatozoa. Biochem. J. 259, 397-406 (1989).

46. Capuano, C., Paolini, R., Molfetta, R., Frati, L., Santoni, A. \& Galandrini, R. PIP2dependent regulation of Munc13-4 endocytic recycling: impact on the cytolytic secretory pathway. Blood 119, 2252-2262 (2012).

47. Stojilkovic, S. S. Ca ${ }^{2+}$-regulated exocytosis and SNARE function. Trends Endocrinol. Metab. 16, 81-83 (2005).

48. Antonescu, C. N., Aguet, F., Danuser, G. \& Schmid, S. L. Phosphatidylinositol$(4,5)$-bisphosphate regulates clathrin-coated pit initiation, stabilization, and size. Mol. Biol. Cell 22, 2588-2600 (2011).

49. Simonsen, A., Wurmser, A. E., Emr, S. D. \& Stenmark, H. The role of phosphoinositides in membrane transport. Curr. Opin. Cell Biol. 13, 485-492 (2001).

50. Leventis, P. A. \& Grinstein, S. The Distribution and Function of Phosphatidylserine in Cellular Membranes. Annu. Rev. Biophys. 39, 407-427 (2010).

51. Reutelingsperger, C. P. M. \& van Heerde, W. L. Annexin V, the regulator of phosphatidylserine-catalyzed inflammation and coagulation during apoptosis. Cell. Mol. Life Sci. 53, 527-532 (1997).

52. Clark, D. P. \& Pazdernik, N. J. Molekulare Biotechnologie: Grundlagen und Anwendungen. Spektrum Akademischer Verlag, Heidelberg (2009), 523-551. 
53. Pérez-Lara, Á., Thapa, A., Nyenhuis, S. B., Nyenhuis, D. A., Halder, P., Tietzel, M., Tittmann, K., Cafiso, D. S. \& Jahn, R. PtdInsP2 and PtdSer cooperate to trap synaptotagmin-1 to the plasma membrane in the presence of calcium. eLife 5, e15886 (2016).

54. Xu, P., Baldridge, R. D., Chi, R. J., Burd, C. G. \& Graham, T. R. Phosphatidylserine flipping enhances membrane curvature and negative charge required for vesicular transport. J. Cell Biol. 202, 875-886 (2013).

55. Sun, Y. \& Drubin, D. G. The functions of anionic phospholipids during clathrinmediated endocytosis site initiation and vesicle formation. J. Cell Sci. 125, 61576165 (2012).

56. van Meer, G. \& de Kroon, A. I. P. M. Lipid map of the mammalian cell. J. Cell Sci. 124, 5-8 (2011).

57. Henriksen, J., Rowat, A. C., Brief, E., Hsueh, Y. W., Thewalt, J. L., Zuckermann, M. J. \& Ipsen J. H. Universal Behavior of Membranes with Sterols. Biophys. J. 90, 16391649 (2006).

58. Nguyen, T. T., Sly, K. L. \& Conboy, J. C. Comparison of the Energetics of Avidin, Streptavidin, NeutrAvidin, and Anti-Biotin Antibody Binding to Biotinylated Lipid Bilayer Examined by Second-Harmonic Generation. Anal. Chem. 84, 201-208 (2012).

59. Hiller, Y., Gershoni, J. M., Bayer, E. A. \& Wilchek, M. Biotin binding to avidin. Oligosaccharide side chain not required for ligand association. Biochem. J. 248, 167-171 (1987).

60. Hiller, Y., Bayer, E. A. \& Wilchek, M. Nonglycosylated avidin. Method. Enzymol 184, 68-70 (1990). 
61. Langel, Ü. Handbook of cell-penetrating peptides. CRC Press, Boca Raton, $2^{\text {nd }}$ edition (2007), 100-102.

62. Moscho, A., Orwar, O., Chiu, D. T., Modi, B. P. \& Zare, R. N. Rapid preparation of giant unilamellar vesicles. Proc. Natl. Acad. Sci. U.S.A. 93, 11443-11447 (1996).

63. Ulrich, A. S. Biophysical aspects of using liposomes as delivery vehicles. Biosci. Rep. 22, 129-150 (2002).

64. Angelova, M. I., Soléau, S., Méléard, Ph., Faucon, F. \& Bothorel, P. Preparation of giant vesicles by external AC electric fields. Kinetics and applications. Prog. Coll. Pol. Sci. S. 89, 127-131 (1992).

65. Horton, H. R., Moran, L. A., Scrimgeour, K. G., Perry, M. D., Rawn, J. D. Biochemie, Pearson Studium, London, $4^{\text {th }}$ edition (2008), 84-86.

66. Gasteiger, E. ExPASy: the proteomics server for in-depth protein knowledge and analysis. Nucleic Acids Res. 31, 3784-3788 (2003).

67. Krick, R., Busse, R. A., Scacioc, A., Stephan, M., Janshoff, A., Thumm, M. \& Kühnel K. Structural and functional characterization of the two phosphoinositide binding sites of PROPPINs, a $\beta$-propeller protein family. Proc. Natl. Acad. Sci. U.S.A. 109, E2042-E2049 (2012).

68. Ludolphs, M., Schneeberger, D., Soykan, T., Schäfer, J., Papadopoulos, T., Brose, N., Schindelin, H. \& Steinem, C. Specificity of Collybistin-Phosphoinositide Interactions: Impact of the Individual Protein Domains. J. Biol. Chem. 291, 244-254 (2016).

69. Gauglitz, G., Brecht, A., Kraus, G. \& Mahm, W. Chemical and biochemical sensors based on interferometry at thin (multi-) layers. Sens. Actuators B 11, 21-27 (1993). 
70. Schiebener, P., Straub, J., Levelt Sengers, J. M. H. \& Gallagher, J. S. Refractive index of water and steam as function of wavelength, temperature and density. $J$. Phys. Chem. Ref. Data 19, 677-717 (1990).

71. Babayan, S. E., Jeong, J. Y., Schütze, A., Tu, V. J., Moravej, M., Selwyn, G. S. \& Hicks, R. F. Deposition of silicon dioxide films with a non-equilibrium atmospheric-pressure plasma jet. Plasma Sources Sci. Technol. 10, 573-578 (2001).

72. Vörös, J. The Density and Refractive Index of Adsorbing Protein Layers. Biophys. J. 87, 553-561 (2004).

73. Walker, J. M. \& Raplay, R. Molecular Biomethods Handbook. (Humana Press, 2008).

74. Cole, R. W., Jinadasa, T. \& Brown, C. M. Measuring and interpreting point spread functions to determine confocal microscope resolution and ensure quality control. Nat. Protoc. 6, 1929-1941 (2011).

75. Macháň, R. \& Hof, M. Lipid diffusion in planar membranes investigated by fluorescence correlation spectroscopy. Biochim. Biophys. Acta Biomembr. 1798, 1377-1391 (2010).

76. Axelrod, D., Koppel, D. E., Schlessinger, J., Elson, E. \& Webb, W. W. Mobility measurement by analysis of fluorescence photobleaching recovery kinetics. Biophys. J. 16, 1055-1069 (1976).

77. Meyvis, T. K. L., De Smedt, S. C., Van Oostveldt, P. \& Demeester, J. Fluorescence Recovery After Photobleaching: A Versatile Tool for Mobility and Interaction Measurements in Pharmaceutical Research. Pharm. Res. 16, 1153-1162 (1999). 
78. Jönsson, P., Jonsson, M. P., Tegenfeldt, J. O. \& Höök, F. A Method Improving the Accuracy of Fluorescence Recovery after Photobleaching Analysis. Biophys. J. 95, $5334-5348$ (2008).

79. Gleisner, M. Interaction of Epsin N-Terminal Homology domain (ENTH) with artificial lipid membranes as a function of lateral tension. Dissertation, University of Göttingen (2016).

80. Binnig, G., Quate, C. F. \& Gerber, Ch. Atomic Force Microscope. Phys. Rev. Lett. 56, 930-933 (1986).

81. Ando, T., Uchihashi, T. \& Scheuring, S. Filming Biomolecular Processes by HighSpeed Atomic Force Microscopy. Chem. Rev. 114, 3120-3188 (2014).

82. Florin, E., Moy, V. \& Gaub, H. Adhesion forces between individual ligand-receptor pairs. Science $\mathbf{2 6 4}, 415-417$ (1994).

83. Das, C., Sheikh, K. H., Olmsted, P. D. \& Connell, S. D. Nanoscale mechanical probing of supported lipid bilayers with atomic force microscopy. Phys. Rev. E 82, (2010).

84. Kopycinska-Müller, M., Geiss, R. H. \& Hurley, D. C. Contact mechanics and tip shape in AFM-based nanomechanical measurements. Ultramicroscopy 106, 466$474(2006)$.

85. Butt, H.-J., Cappella, B. \& Kappl, M. Force measurements with the atomic force microscope: Technique, interpretation and applications. Surf. Sci. Rep. 59, 1-152 (2005).

86. Unsay, J. D., Cosentino, K. \& García-Sáez, A. J. Atomic Force Microscopy Imaging and Force Spectroscopy of Supported Lipid Bilayers. J. Vis. Exp. 101, e52867 (2015). 
87. Sader, J. E., Larson, I., Mulvaney, P. \& White, L. R. Method for the calibration of atomic force microscope cantilevers. Rev. Sci. Instrum. 66, 3789 (1995).

88. Kučerka, N., Nieh, M.-P. \& Katsaras, J. Fluid phase lipid areas and bilayer thicknesses of commonly used phosphatidylcholines as a function of temperature. Biochim. Biophys. Acta Biomembr. 1808, 2761-2771 (2011).

89. Roberts, G. G. \& Pitt, C. W. Langmuir-Blodgett Films, 1982. (Elsevie S\&T, 1983).

90. Petty, M. C. Langmuir-Blodgett films: An introduction. (Cambridge University Press, 1996). doi:10.1017/CB09780511622519.

91. Larsen, M. C. Binary Phase Diagrams at the Air-Water Interface: An Experiment for Undergraduate Physical Chemistry Students. J. Chem. Educ. 91, 597-601 (2014).

92. Faye, N. R., Gammoudi, I., Moroté, F., Grauby-Heywang, C. \& Cohen-Bouhacina, T. Phase Behavior And Structure Properties Of Supported Lipid Monolayers And Bilayers In Interaction With Silica Nanoparticles. Int. J. Biotechnol. Bioeng. 7, 441448 (2013).

93. Velázquez, M. M., Alejo, T., López-Díaz, D., Martín-García, B. \& Merchán, M. D. Langmuir-Blodgett Methodology: A Versatile Technique to Build 2D Material Films. in Two-dimensional Materials - Synthesis, Characterization and Potential Applications (ed. Nayak, P. K.) (InTech, 2016). doi:10.5772/63495.

94. Mollet, H. \& Grubenmann, A. Formulierungstechnik: Emulsionen, Suspensionen, feste Formen. Wiley-VCH, Weinheim (2000), 23-30.

95. Vargaftik, N. B., Volkov, B. N. \& Voljak, L. D. International Tables of the Surface Tension of Water. J. Phys. Chem. Ref. Data 12, 817-820 (1983). 
96. Dennis, J., Briant, J., Hipeaux, J. Lubricant Properties Analysis and Testing. Ind. Lubrication and Tribology 52, 248-248 (2000).

97. Braunger, J. A., Kramer, C., Morick, D. \& Steinem, C. Solid Supported Membranes Doped with PIP 2 : Influence of Ionic Strength and pH on Bilayer Formation and Membrane Organization. Langmuir 29, 14204-14213 (2013).

98. Bradshaw, J. P., Bushby, R. J., Giles, C. C. D. \& Saunders, M. R. Orientation of the Headgroup of Phosphatidylinositol in a Model Biomembrane As Determined by Neutron Diffraction. Biochemistry 38, 8393-8401 (1999).

99. Richter, R. P., Maury, N. \& Brisson, A. R. On the Effect of the Solid Support on the Interleaflet Distribution of Lipids in Supported Lipid Bilayers. Langmuir 21, 299-304 (2005).

100. Scomparin, C., Lecuyer, S., Ferreira, M., Charitat, T. \& Tinland, B. Diffusion in supported lipid bilayers: Influence of substrate and preparation technique on the internal dynamics. Eur. Phys. J. E 28, 211-220 (2009).

101. Witte, L. On the derivation of the Langmuir adsorption isotherms. Kolloid Z. Z. Polym. 202, 147-153 (1965).

102. Dada, A. O., Olalekan, A. P., Olatunya, A. M. \& Dada, O. Langmuir, Freundlich, Temkin and Dubinin-Radushkevich Isotherms Studies of Equilibrium Sorption of Zn 2+ Unto Phosphoric Acid Modified Rice Husk. IOSR J. Appl. Chem. 3, 38-45 (2012).

103. Levenberg, K. A method for the solution of certain non-linear problems in least squares. Quart. Appl. Math. 2, 164-168 (1944).

104. Marquardt, D. W. An Algorithm for Least-Squares Estimation of Nonlinear Parameters. J. Soc. Indust. Appl. Math. 11, 431-441 (1963). 
105. Shouren Ge, Kojio, K., Takahara, A. \& Kajiyama, T. Bovine serum albumin adsorption onto immobilized organotrichlorosilane surface: Influence of the phase separation on protein adsorption patterns. J. Biomater. Sci. Polymer Edn. 9, 131150 (1998).

106. Coen, M. C., Lehmann, R., Gröning, P., Bielmann, M., Galli, C. \& Schlapbach, L. Adsorption and Bioactivity of Protein A on Silicon Surfaces Studied by AFM and XPS. J. Colloid Interface Sci. 233, 180-189 (2001).

107. Gumí-Audenis, B., Sanz, F. \& Giannotti, M. I. Impact of galactosylceramides on the nanomechanical properties of lipid bilayer models: an AFM-force spectroscopy study. Soft Matter 11, 5447-5454 (2015).

108. Freedman, D. \& Diaconis, P. On the histogram as a density estimator:L 2 theory. Z. Wahrscheinlichkeitstheorie verw. Gebiete 57, 453-476 (1981).

109. García, R. Amplitude Modulation Atomic Force Microscopy. Wiley-VCH Verlag, Weinheim, (2010), 103-114.

110. Lai, C.-L., Jao, C. C., Lyman, E., Gallop, J. L., Peter, B. J., McMahon, H. T., Langen R. \& Voth, G. A. Membrane Binding and Self-Association of the Epsin N-Terminal Homology Domain. J. Mol. Biol. 423, 800-817 (2012).

111. Arnett, E. M., Harvey, N. G. \& Rose, P. L. Stereochemistry and molecular recognition in 'two dimensions'. Acc. Chem. Res. 22, 131-138 (1989).

112. Kumar, R. \& Manjuladevi, V. Molecular Interactions at Interfaces. in Molecular Interactions (ed. Meghea, A.) (InTech, 2012). doi:10.5772/35628.

113. Demel, R. A., Geurts van Kessel, W. S. M., Zwaal, R. F. A., Roelofsen, B. \& van Deenen, L. L. M. Relation between various phospholipase actions on human red cell 
membranes and the interfacial phospholipid pressure in monolayers. Biochim. Biophys. Acta Biomembr. 406, 97-107 (1975).

114. Blume, A. A comparative study of the phase transitions of phospholipid bilayers and monolayers. Biochim. Biophys. Acta Biomembr. 557, 32-44 (1979).

115. Johnston, D. S., Coppard, E., Parera, G. V. \& Chapman, D. Langmuir film balance study of the interactions between carbohydrates and phospholipid monolayers. Biochemistry 23, 6912-6919 (1984).

116. Fenz, S. F. \& Sengupta, K. Giant vesicles as cell models. Integr. Biol. 4, 982 (2012).

117. Marsh, D. Renormalization of the tension and area expansion modulus in fluid membranes. Biophys. J. 73, 865-869 (1997).

118. Helfrich, W. \& Servuss, R.-M. Undulations, steric interaction and cohesion of fluid membranes. Nuovo Cimento D 3, 137-151 (1984).

119. Evans, E. \& Rawicz, W. Entropy-driven tension and bending elasticity in condensed-fluid membranes. Phys. Rev. Lett. 64, 2094-2097 (1990).

120. Rawicz, W., Olbrich, K. C., McIntosh, T., Needham, D. \& Evans, E. Effect of Chain Length and Unsaturation on Elasticity of Lipid Bilayers. Biophys. J. 79, 328-339 (2000).

121. Rädler, J. O., Feder, T. J., Strey, H. H. \& Sackmann, E. Fluctuation analysis of tension-controlled undulation forces between giant vesicles and solid substrates. Phys. Rev. E 51, 4526-4536 (1995).

122. Chiaruttini, N., Redondo-Morata, L., Colom, A., Humbert, F., Lenz, M., Scheuring, S. \& Roux, A. Relaxation of Loaded ESCRT-III Spiral Springs Drives Membrane Deformation. Cell 163, 866-879 (2015). 
123. Bhatia, T., Husen, P., Ipsen, J. H., Bagatolli, L. A. \& Simonsen, A. C. Fluid domain patterns in free-standing membranes captured on a solid support. Biochim. Biophys. Acta Biomembr. 1838, 2503-2510 (2014).

124. Sakuma, Y., Imai, M., Yanagisawa, M. \& Komura, S. Adhesion of binary giant vesicles containing negative spontaneous curvature lipids induced by phase separation. Eur. Phys. J. E 25, 403 (2008).

125. Roldán-Vargas, S., Mártin-Molina, A., Quesada-Pérez, M., Barnadas-Rodríguez, R., Estelrich, J. \& Callejas-Fernández, J. Aggregation of liposomes induced by calcium: A structural and kinetic study. Phys. Rev. E 75, 021912 (2007).

126. Schultz, Z. D., Pazos, I. M., McNeil-Watson, F. K., Lewis, E. N. \& Levin, I. W. Magnesium-Induced Lipid Bilayer Microdomain Reorganizations: Implications for Membrane Fusion. J. Phys. Chem. B 113, 9932-9941 (2009).

127. Meyer, S. C., Gaj, T. \& Ghosh, I. Highly Selective Cyclic Peptide Ligands for NeutrAvidin and Avidin Identified by Phage Display. Chem. Biol. Drug Des. 68, 3-10 (2006).

128. Schwamborn, M., Schumacher, J., Sibold, J., Teiwes, N. K. \& Steinem, C. Monitoring ATPase induced $\mathrm{pH}$ changes in single proteoliposomes with the lipid-coupled fluorophore Oregon Green 488. Analyst 142, 2670-2677 (2017).

129. Piehler, J., Brecht, A., Valiokas, R., Liedberg, B. \& Gauglitz, G. A high-density poly(ethylene glycol) polymer brush for immobilization on glass-type surfaces. $B i$ osens. Bioelectron. 15, 473-481 (2000).

130. Åkesson, A., Lind, T., Ehrlich, N., Stamou, D., Wacklin, H. \& Cárdenas, M. Composition and structure of mixed phospholipid supported bilayers formed by POPC and DPPC. Soft Matter 8, 5658 (2012). 
131. Alwarawrah, M., Dai, J. \& Huang, J. A Molecular View of the Cholesterol Condensing Effect in DOPC Lipid Bilayers. J. Phys. Chem. B 114, 7516-7523 (2010).

132. Kučerka, N., Tristram-Nagle, S. \& Nagle, J. F. Structure of Fully Hydrated Fluid Phase Lipid Bilayers with Monounsaturated Chains. J. Membrane Biol. 208, 193202 (2006).

133. Subramaniam, V., D'Ambruoso, G. D., Hall, H. K. Jr., Wysocki, R. J. Jr., Brown, M. F. \& Saavedra, S. S. Reconstitution of rhodopsin into polymerizable planar supported lipid bilayers: Influence of dienoyl monomer structure on photoactivation. Langmuir 24, 11067-11075 (2008).

134. Kramer, C. Verknüpfung zwischen Plasmamembran und Zytoskelett: Charakterisierung der Organisation von Ezrin und F-Aktin an artifiziellen Lipidmembranen. Dissertation, University of Göttingen (2016).

135. Rossi, C., Homand, J., Bauche, C., Hamdi, H., Ladant, D. \& Chopineau, J. Differential Mechanisms for Calcium-Dependent Protein/Membrane Association as Evidenced from SPR-Binding Studies on Supported Biomimetic Membranes †. Biochemistry 42, 15273-15283 (2003).

136. Macháň, R. \& Hof, M. Recent Developments in Fluorescence Correlation Spectroscopy for Diffusion Measurements in Planar Lipid Membranes. Int. J. Mol. Sci. 11, 427-457 (2010).

137. König, B. W., Krueger, S., Orts, W. J., Majkrzak, C. F., Berk, N. F., Silverton, J. V. \& Gawrisch, K. Neutron reflectivity and atomic force microscopy studies of a lipid bilayer in water adsorbed to the surface of a silicon single crystal. Langmuir 12, 1343-1350 (1996). 
138. Kim, J., Kim, G. \& Cremer, P. S. Investigations of Water Structure at the Solid/Liquid Interface in the Presence of Supported Lipid Bilayers by Vibrational Sum Frequency Spectroscopy. Langmuir 17, 7255-7260 (2001).

139. Bayerl, T. M. \& Bloom, M. Physical properties of single phospholipid bilayers adsorbed to micro glass beads. A new vesicular model system studied by $2 \mathrm{H}-\mathrm{nu}-$ clear magnetic resonance. Biophys. J. 58, 357-362 (1990).

140. Seu, K. J., Pandey, A. P., Haque, F., Proctor, E. A., Ribbe \& A. E., Hovis, J. S. Effect of Surface Treatment on Diffusion and Domain Formation in Supported Lipid Bilayers. Biophys. J. 92, 2445-2450 (2007).

141. Tamm, L. K. \& McConnell, H. M. Supported phospholipid bilayers. Biophys. J. 47, 105-113 (1985).

142. Lindblom, G. \& Orädd, G. Lipid lateral diffusion and membrane heterogeneity. Biochim. Biophys. Acta Biomembr. 1788, 234-244 (2009).

143. Baumann, M. K., Amstad, E., Mashaghi, A., Textor, M. \& Reimhult, E. Characterization of supported lipid bilayers incorporating and phosphoinositol-3,4,5-triphosphate by complementary techniques. Biointerphases 5, 114-119 (2010).

144. Zhang, Z., Nadezhina, E. \& Wilkinson, K. J. Quantifying Diffusion in a Biofilm of Streptococcus mutans. Antimicrob. Agents Chemother. 55, 1075-1081 (2011).

145. Kweon, D.-H., Shin, Y-K., Shin, J. Y., Lee, J.-H., Lee, J.-B., Seo, J.-H. \& Kim, Y. S. Membrane topology of helix 0 of the Epsin N-terminal homology domain. Mol. Cells 21, 428-435 (2006).

146. Feder, J. \& Giaever, I. Adsorption of ferritin. J. Colloid Interface Sci. 78, 144-154 (1980). 
147. Li, Z., Venable, R. M., Rogers, L. A., Murray, D. \& Pastor, R. W. Molecular dynamics simulations of PIP2 and PIP3 in lipid bilayers: determination of ring orientation, and the effects of surface roughness on a Poisson-Boltzmann description. Biophys. J. 97, 155-163 (2009).

148. Czolkos, I., Jesorka, A. \& Orwar, O. Molecular phospholipid films on solid supports. Soft Matter 7, 4562-4576 (2011).

149. Sanghera, N., Swann, M. J., Ronan, G. \& Pinheiro, T. J. T. Insight into early events in the aggregation of the prion protein on lipid membranes. Biochim. Biophys. Acta Biomembr. 1788, 2245-2251 (2009).

150. Llopis, J., McCaffery, J. M., Miyawaki, A., Farquhar, M. G. \& Tsien, R. Y. Measurement of cytosolic, mitochondrial, and Golgi $\mathrm{pH}$ in single living cells with green fluorescent proteins. Proc. Natl. Acad. Sci. U.S.A. 95, 6803-6808 (1998).

151. Gleisner, M., Mey, I., Barbot, M., Dreker, C., Meinecke, M. \& Steinem, C. Driving a planar model system into the $3^{\text {rd }}$ dimension: generation and control of curved pore-spanning membrane arrays. Soft Matter 10, 6228-6236 (2014).

152. Baumgart, T., Capraro, B. R., Zhu, C. \& Das, S. L. Thermodynamics and Mechanics of Membrane Curvature Generation and Sensing by Proteins and Lipids. Annu. Rev. Phys. Chem. 62, 483-506 (2011).

153. Cornell, R. \& Taneva, S. Amphipathic Helices as Mediators of the Membrane Interaction of Amphitropic Proteins, and as Modulators of Bilayer Physical Properties. Curr. Protein Pept. Sci. 7, 539-552 (2006).

154. Snead, W. T., Hayden, C. C., Gadok, A. K., Zhao, C., Lafer, E. M., Rangamani, P. \& Stachowiak, J. C. Membrane fission by protein crowding. Proc. Natl. Acad. Sci. U.S.A. 114, E3258-E3267 (2017). 
155. Tsujita, K., Suetsugu, S., Sasaki, N., Furutani, M., Oikawa, T. \& Takenawa, T. Coordination between the actin cytoskeleton and membrane deformation by a novel membrane tubulation domain of PCH proteins is involved in endocytosis. J. Cell Biol. 172, 269-279 (2006).

156. Hyman, J., Chen, H., Fiore, P. P. D., Camilli, P. D. \& Brunger, A. T. Epsin 1 Undergoes Nucleocytosolic Shuttling and its Eps15 Interactor NH2-terminal Homology (ENTH) Domain, Structurally Similar to Armadillo and HEAT Repeats, Interacts with the Transcription Factor Promyelocytic Leukemia $\mathrm{Zn}^{2+}$ Finger Protein (PLZF). J. Cell Biol. 149, 10 (2000).

157. Milhiet, P.-E., Giocondi, M.-C., Baghdadi, O., Grimellec, C. L. \& Roux, B. AFM Detection of GPI Protein Insertion into DOPC/DPPC Model Membranes. Single Mol. 3, 135-140 (2002).

158. Milhiet, P.-E., Giocondi, M-C., Baghdadi, O., Ronzon, F., Roux, B. \& Le Grimellec, C. Spontaneous insertion and partitioning of alkaline phosphatase into model lipid rafts. EMBO Rep. 3, 485-490 (2002).

159. Hsieh, C.-L., Spindler, S., Ehrig, J. \& Sandoghdar, V. Tracking Single Particles on Supported Lipid Membranes: Multimobility Diffusion and Nanoscopic Confinement. J. Phys. Chem. B 118, 1545-1554 (2014).

160. Przybylo, M., Sýkora, J., Humpolíčková, J., Benda, A., Zan, A. \& Hof, M. Lipid Diffusion in Giant Unilamellar Vesicles Is More than 2 Times Faster than in Supported Phospholipid Bilayers under Identical Conditions. Langmuir 22, 9096-9099 (2006). 
161. Solanko, L. M., Honigmann, A., Midtiby, H. S., Lund, F. W., Brewer, J. R., Dekaris,

V., Bittmann, R., Eggeling, C. \& Wüstner, D. Membrane Orientation and Lateral Diffusion of BODIPY-Cholesterol as a Function of Probe Structure. Biophys. J. 105, 2082-2092 (2013).

162. Sharpe, H. J., Stevens, T. J. \& Munro, S. A Comprehensive Comparison of Transmembrane Domains Reveals Organelle-Specific Properties. Cell 142, 158-169 (2010).

163. Gilmanshin, R., Creutz, C. E. \& Tamm, L. K. Annexin IV Reduces the Rate of Lateral Lipid Diffusion and Changes the Fluid Phase Structure of the Lipid Bilayer When It Binds to Negatively Charged Membranes in the Presence of Calcium. Biochemistry 33, 8225-8232 (1994).

164. Rozovsky, S., Forstner, M. B., Sondermann, H. \& Groves, J. T. Single Molecule Kinetics of ENTH Binding to Lipid Membranes. J. Phys. Chem. B 116, 5122-5131 (2012).

165. Forstner, M. B., Yee, C. K., Parikh, A. N. \& Groves, J. T. Lipid Lateral Mobility and Membrane Phase Structure Modulation by Protein Binding. J. Am. Chem. Soc. 128, 15221-15227 (2006).

166. Zhong, Q., Inniss, D., Kjoller, K. \& Elings, V. B. Fractured polymer/silica fiber surface studied by tapping mode atomic force microscopy. Surf. Sci. Lett. 290, L688-L692 (1993).

167. Abramson, M. B., Colacicco, G., Curci, R. \& Rapport, M. M. Ionic properties of acidic lipids. Phosphatidylinositol. Biochemistry 7, 1692-1698 (1968). 
168. Neves, B. R. A., Leonard, D. N., Salmon, M. E., Russell, P. E. \& Troughton, E. B. Observation of topography inversion in atomic force microscopy of self-assembled monolayers. Nanotechnology 10, 399-404 (1999).

169. Palacios-Lidón, E., Munuera, C., Ocal, C. \& Colchero, J. Contrast inversion in noncontact Dynamic Scanning Force Microscopy: What is high and what is low? Ultramicroscopy 110, 789-800 (2010).

170. Rahe, P., Bechstein, R., Schütte, J., Ostendorf, F. \& Kühnle, A. Repulsive interaction and contrast inversion in noncontact atomic force microscopy imaging of adsorbates. Phys. Rev. B 77, 195410 (2008).

171. Reiss, H., Frisch, H. L. \& Lebowitz, J. L. Statistical Mechanics of Rigid Spheres. J. Chem. Phys. 31, 369-380 (1959).

172. Rakotomanga, M., Saint-Pierre-Chazalet, M. \& Loiseau, P. M. Alteration of Fatty Acid and Sterol Metabolism in Miltefosine-Resistant Leishmania donovani Promastigotes and Consequences for Drug-Membrane Interactions. Antimicrob. Agents Chemother. 49, 2677-2686 (2005).

173. Levental, I., Janmey, P. A. \& Cēbers, A. Electrostatic Contribution to the Surface Pressure of Charged Monolayers Containing Polyphosphoinositides. Biophys. J. 95, 1199-1205 (2008).

174. Hermelink, A. \& Brezesinski, G. Do unsaturated phosphoinositides mix with ordered phosphadidylcholine model membranes? J. Lipid Res. 49, 1918-1925 (2008).

175. Gramlich, G., Zhang, J., Winterhalter, M. \& Nau, W. M. A long-lived amphiphilic fluorescent probe studied in POPC air-water monolayer and solution bilayer systems. Chem. Phys. Lipids 113, 1-9 (2001). 
176. Redfern, D. A. \& Gericke, A. pH-dependent domain formation in phosphatidylinositol polyphosphate/phosphatidylcholine mixed vesicles. J. Lipid Res. 46, 504515 (2005).

177. Kooijman, E. E., King, K. E., Gangoda, M. \& Gericke, A. Ionization Properties of Phosphatidylinositol Polyphosphates in Mixed Model Membranes. Biochemistry 48, 9360-9371 (2009).

178. Domènech, Ò., Sanz, F., Montero, M. T. \& Hernández-Borrell, J. Thermodynamic and structural study of the main phospholipid components comprising the mitochondrial inner membrane. Biochim. Biophys. Acta Biomembr. 1758, 213-221 (2006).

179. Garcia-Manyes, S., Domènech, Ò., Sanz, F., Montero, M. T. \& Hernandez-Borrell, J. Atomic force microscopy and force spectroscopy study of Langmuir-Blodgett films formed by heteroacid phospholipids of biological interest. Biochim. Biophys. Acta Biomembr. 1768, 1190-1198 (2007).

180. Birdi, K. S. \& Vu, D. T. Structures of Collapsed Lipid Monolayers Investigated as Langmuir-Blodgett Films by Atomic Force Microscopy. Langmuir 10, 623-625 (1994).

181. Boucrot, E., Pick, A., Çamdere, G., Liska, N., Evergren, E., McMahon, H. T. \& Kozlov, M. M. Membrane Fission Is Promoted by Insertion of Amphipathic Helices and Is Restricted by Crescent BAR Domains. Cell 149, 124-136 (2012).

182. Stahelin, R. V., Long, F., Diraviyam, K., Bruzik, K. S., Murray, D. \& Cho, W. Phosphatidylinositol 3-Phosphate Induces the Membrane Penetration of the FYVE Domains of Vps27p and Hrs. J. Biol. Chem. 277, 26379-26388 (2002). 
183. Hussain, H., Kerth, A., Blume, A. \& Kressler, J. Amphiphilic Block Copolymers of Poly(ethylene oxide) and Poly(perfluorohexylethyl methacrylate) at the Water Surface and Their Penetration into the Lipid Monolayer.J. Phys. Chem. B 108, 99629969 (2004).

184. Maget-Dana, R. The monolayer technique: a potent tool for studying the interfacial properties of antimicrobial and membrane-lytic peptides and their interactions with lipid membranes. Biochim. Biophys. Acta Biomembr. 1462, 109-140 (1999).

185. Fainerman, V. B. \& Vollhardt, D. Penetration of Langmuir Monolayers by Soluble Amphiphilic Molecules. Langmuir 15, 1784-1790 (1999).

186. Marsh, D. Lateral pressure in membranes. Biochim. Biophys. Acta Rev. Biomembr. 1286, 183-223 (1996).

187. Ulman, A. An introduction to ultrathin organic films: from Langmuir-Blodgett to self-assembly. Academic Press, Cambridge, $1^{\text {st }}$ edition (1991), 102-130.

188. Verger, R. \& Pattus, F. Lipid-protein interactions in monolayers. Chem. Phys. Lipids 30, 189-227 (1982).

189. Subirade, M., Salesse, C., Marion, D. \& Pézolet, M. Interaction of a nonspecific wheat lipid transfer protein with phospholipid monolayers imaged by fluorescence microscopy and studied by infrared spectroscopy. Biophys. J. 69, 974-988 (1995).

190. Ishitsuka, Y., Pham, D. S., Waring, A. J., Lehrer, R. I. \& Lee, K. Y. C. Insertion selectivity of antimicrobial peptide protegrin-1 into lipid monolayers: Effect of head group electrostatics and tail group packing. Biochim. Biophys. Acta Biomembr. 1758, 1450-1460 (2006). 
191. Schafer, L. V., de Jong, D. H., Holt, A., Rzepiela, A. J., de Vries, A. H., Poolman, B., Killian, J. A. \& Marrink, S. J. Lipid packing drives the segregation of transmembrane helices into disordered lipid domains in model membranes. Proc. Natl. Acad. Sci. U.S.A. 108, 1343-1348 (2011).

192. Lucas, N. \& Cho, W. Phosphatidylserine Binding Is Essential for Plasma Membrane Recruitment and Signaling Function of 3-Phosphoinositide-dependent Kinase-1. J. Biol. Chem. 286, 41265-41272 (2011).

193. Johnson, K. A., Bhattarai, N., Budicini, M. R., Shirey, C. M., Baker, S. C. B., Gerstman, B. S., Chapagain, P. P. \& Stahelin, R. V. Mutation of Ebola Virus Matrix Protein Cysteine Residues Increases Binding to Phosphatidylserine through Increased Flexibility of a Lipid Binding Loop. bioRxiv 286120 (2018).

194. Israelachvili, J. N. \& Mitchell, D. J. A model for the packing of lipids in bilayer membranes. Biochim. Biophys. Acta Biomembr. 389, 13-19 (1975).

195. Atkinson, D., Hauser, H., Shipley, G. G. \& Stubbs, J. M. Structure and morphology of phosphatidylserine dispersions. Biochim. Biophys. Acta Biomembr. 339, 10-29 (1974).

196. Vanni, S., Hirose, H., Barelli, H., Antonny, B. \& Gautier, R. A sub-nanometre view of how membrane curvature and composition modulate lipid packing and protein recruitment. Nat. Commun. 5, 4916 (2014).

197. Bigay, J., Casella, J.-F., Drin, G., Mesmin, B. \& Antonny, B. ArfGAP1 responds to membrane curvature through the folding of a lipid packing sensor motif. EMBO J. 24, 2244-2253 (2005). 
198. Risselada, H. J. \& Marrink, S. J. Curvature effects on lipid packing and dynamics in liposomes revealed by coarse grained molecular dynamics simulations. Phys. Chem. Chem. Phys. 11, 2056 (2009).

199. Drin, G., Casella, J.-F., Gautier, R., Boehmer, T., Schwartz, T. U. \& Antonny, B. A general amphipathic $\alpha$-helical motif for sensing membrane curvature. Nat. Struct. Mol. Biol. 14, 138-146 (2007).

200. Evans, E. A. \& Parsegian, V. A. Energetics of Membrane Deformation and Adhesion in Cell and Vesicle Aggregation. Ann. NY Acad. Sci. 416, 13-33 (1983).

201. Gauthier, N. C., Masters, T. A. \& Sheetz, M. P. Mechanical feedback between membrane tension and dynamics. Trends Cell Biol. 22, 527-535 (2012).

202. Spencer, N. D. Tailoring surfaces: modifying surface composition and structure for applications in tribology, biology and catalysis. World Scientific, Singapur, (2011).

203. Zocchi, A., Marya Jobé, A., Neuhaus, J.-M. \& Ward, T. R. Expression and purification of a recombinant avidin with a lowered isoelectric point in Pichia pastoris. Protein Expr. Purif. 32, 167-174 (2003).

204. Thiart, J. Probing Lipid Diffusion in Curved and Planar Membranes with Fluorescence Microscopy, Dissertation, University of Göttingen (2017).

205. Kaasgaard, T., Mouritsen, O. G. \& Jørgensen, K. Screening effect of PEG on avidin binding to liposome surface receptors. Int. J. Pharm. 214, 63-65 (2001).

206. Etienne, O., Taddei, C. P., Haikel, Y., Dimarcq, J. L., Schaaf, P., Voegel, J. C., Ogier, J. A. \& Egels, C. Multilayer Polyelectrolyte Films Functionalized by Insertion of Defensin: a New Approach to Protection of Implants from Bacterial Colonization. Antimicrob. Agents Chemother. 48, 3662-3669 (2004). 
207. Hu, Y., Jin, J., Han, Y., Yin, J., Jiang, W. \& Liang, H. Study of fibrinogen adsorption on poly(ethylene glycol)-modified surfaces using a quartz crystal microbalance with dissipation and a dual polarization interferometry. RSC Adv. 4, 7716 (2014).

208. Dai, J. \& Sheetz, M. P. Membrane Tether Formation from Blebbing Cells. Biophys. J. 77, 3363-3370 (1999).

209. Lieber, A. D., Yehudai-Resheff, S., Barnhart, E. L., Theriot, J. A. \& Keren, K. Membrane Tension in Rapidly Moving Cells Is Determined by Cytoskeletal Forces. Curr. Biol. 23, 1409-1417 (2013).

210. Pietuch, A., Brückner, B. R. \& Janshoff, A. Membrane tension homeostasis of epithelial cells through surface area regulation in response to osmotic stress. Biochim. Biophys. Acta Mol. Cell Res. 1833, 712-722 (2013).

211. Raucher, D., Stauffer, T., Chen, W., Shen, K., Guo, S., York, J. D., Sheetz, M., P. \& Meyer T. Phosphatidylinositol 4,5-Bisphosphate Functions as a Second Messenger that Regulates Cytoskeleton-Plasma Membrane Adhesion. Cell 100, 221-228 (2000).

212. Hubrich, R., Park, Y., Mey, I., Jahn, R. \& Steinem, C. SNARE-Mediated Fusion of Single Chromaffin Granules with Pore-Spanning Membranes. Biophys. J. 116, 308318 (2019).

213. Steinkühler, J., De Tillieux, P., Knorr, R. L., Lipowsky, R. \& Dimova, R. Charged giant unilamellar vesicles prepared by electroformation exhibit nanotubes and transbilayer lipid asymmetry. Sci. Rep. 8, 11838 (2018).

214. Montes, L.-R., Alonso, A., Goñi, F. M. \& Bagatolli, L. A. Giant Unilamellar Vesicles Electroformed from Native Membranes and Organic Lipid Mixtures under Physiological Conditions. Biophys. J. 93, 3548-3554 (2007). 
215. Schmid, E. M., Richmond, D. L. \& Fletcher, D. A. Reconstitution of proteins on electroformed giant unilamellar vesicles. Methods Cell Biol. 128, 319-338 (2015).

216. Bacia, K., Futai, E., Prinz, S., Meister, A., Daum, S., Glatte, D., Briggs, J. A. G. \& Schekman, R. Multibudded tubules formed by COPII on artificial liposomes. Sci. Rep. 1, 17 (2011).

217. Antonny, B. Membrane deformation by protein coats. Curr. Opin. Cell Biol. 18, 386-394 (2006).

218. Cui, H., Lyman, E. \& Voth, G. A. Mechanism of Membrane Curvature Sensing by Amphipathic Helix Containing Proteins. Biophys. J. 100, 1271-1279 (2011).

219. Hatzakis, N. S., Bhatia, V. K., Larsen, J., Madsen, K. L., Bolinger, P.-Y., Kunding, A. H., Castillo, J., Gether, U., Hedegård, P. \& Stamou D. How curved membranes recruit amphipathic helices and protein anchoring motifs. Nature Chemical Biology 5, 835841 (2009).

220. Nuscher, B., Kamp, F., Mehnert, T., Odoy, S., Haass, C., Kahle, P. J. \& Beyer, K. $\alpha-$ Synuclein Has a High Affinity for Packing Defects in a Bilayer Membrane: A Thermodynamics Study. J. Biol. Chem. 279, 21966-21975 (2004).

221. Capraro, B. R., Yoon, Y., Cho, W. \& Baumgart, T. Curvature Sensing by the Epsin N-Terminal Homology Domain Measured on Cylindrical Lipid Membrane Tethers. J. Am. Chem. Soc. 132, 1200-1201 (2010).

222. Bezlyepkina, N., Gracià, R. S., Shchelokovskyy, P., Lipowsky, R. \& Dimova, R. Phase Diagram and Tie-Line Determination for the Ternary Mixture DOPC/eSM/Cholesterol. Biophys. J. 104, 1456-1464 (2013). 
223. Baykal-Caglar, E., Hassan-Zadeh, E., Saremi, B. \& Huang, J. Preparation of giant unilamellar vesicles from damp lipid film for better lipid compositional uniformity. Biochim. Biophys. Acta Biomembr. 1818, 2598-2604 (2012).

224. Krishnan, K., Holub, O., Gratton, E., Clayton, A. H. A., Cody, S. \& Moens, P. D. J. Profilin Interaction with Phosphatidylinositol $(4,5)$-Bisphosphate Destabilizes the Membrane of Giant Unilamellar Vesicles. Biophysical Journal 96, 5112-5121 (2009).

225. Brückner, E., Sonntag, P. \& Rehage, H. Light-Induced Shape Transitions of Unilamellar Vesicles. Langmuir 17, 2308-2311 (2001).

226. Käs, J. \& Sackmann, E. Shape transitions and shape stability of giant phospholipid vesicles in pure water induced by area-to-volume changes. Biophys. J. 60, 825844 (1991). 


\section{LIST OF FIGURES}

Figure 1.1: Schematic illustration of the clathrin-mediated endocytosis.........................

Figure 1.2: Schematic structure of epsin 1 with all important binding motifs.................3

Figure 1.3: Crystal structures of unbound ENTH and the ENTH-IP 3 complex................4

Figure 1.4: Schematic illustration of the predicted mechanism of ENTH induced membrane curvature........................................................................................

Figure 3.1: Schematic drawing of a GUV electro-formation chamber.............................17

Figure 3.2: Hydrophobic functionalization of silicon dioxide wafers with HMDS......19

Figure 3.3: Silanization and PEGylation of glass substrates...........................................2 20

Figure 3.4: Schematic illustration of the Snellius reflaction law...................................... 22

Figure 3.5: Schematic light pathway during a RIfS experiment.......................................23

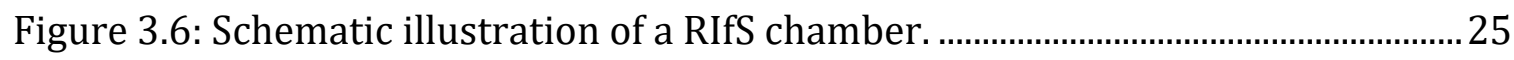

Figure 3.7: Schematic illustration of a confocal laser scanning microscope setup.....27

Figure 3.8: Schematic illustration of a FRAP experiment.................................................29

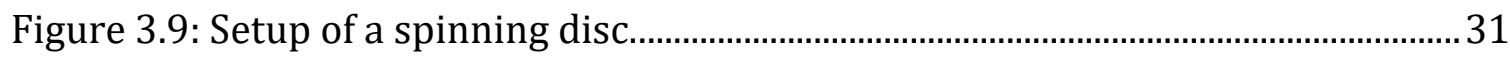

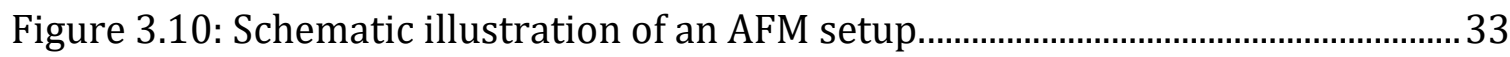

Figure 3.11: Schematic illustration of a signal versus piezo position (A) and a force-

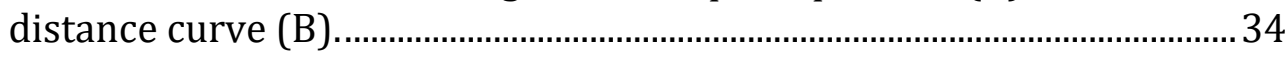

Figure 3.12: Schematic illustration of a Langmuir-Blodgett trough and a typical surface pressure-area per molecule isotherm...............................................37

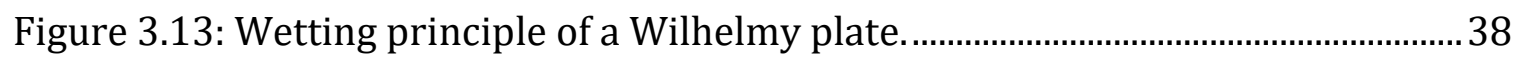

Figure 3.14: Schema of the used protein trough............................................................... 40

Figure 4.1: Exemplary plots of the change in optical thickness vs. the time during vesicle spreading of (A) pure POPC and (B) POPC/PIP 2 (90:10) SUVs onto hydrophilic silicon dioxide wafers.......................................................... 42

Figure 4.2: Time-trace of the spreading process of POPC/PIP 2 -SUVs. ........................... 43 
Figure 4.3: (A) Fluorescence images of a FRAP experiment and (B) the corresponding time-trace of the normalized fluorescence intensity on a POPC/PIP2/TxR (89.5:10:0.5) bilayer.

Figure 4.4: Exemplary RIfS time-traces of the vesicle spreading on hydrophobic functionalized silicon dioxide.

Figure 4.5: (A) Fluorescence images of a FRAP experiment and (B) the corresponding time-trace of the normalized fluorescence intensity on a POPC/PIP2/TxR (89.5:10:0.5) monolayer.

Figure 4.6: Schematic illustration of a RIfS experiment.

Figure 4.7: RIfS time-trace of the ENTH incubation $(1 \mu \mathrm{M})$ to pure POPC $(\mathrm{A})$ bi- and

(B) monolayers.

Figure 4.8: Representative time-resolved change in optical thickness observed by means of RIfS.

Figure 4.9: Time-resolved increase of $\triangle O T$ upon binding of $1 \mu \mathrm{M}$ ENTH to POPC (A) monolayers and (B) bilayers doped with $\mathrm{PIP}_{2}$ (0-10 mol\%). (C) Determined maximum changes in optical thickness after ENTH addition obtained from (A) and (B) plotted against the PIP 2 content.... 54

Figure 4.10: Reversibility of the ENTH binding to POPC/PIP 2 bi- and monolayers as a function of the $\mathrm{PIP}_{2}$ concentration.

Figure 4.11: Exemplary time-trace of (A) POPC/POPS (80:20) and (B) POPC/POPS/PIP2 (75:20:5) vesicles spreading on hydrophilic silicon dioxide substrates.

Figure 4.12: Fluorescence images of (A) POPC/POPS/TxR (79.5:20:0.5) and (B) POPC/POPS/PIP2/TxR (74.9:20:5:0.5) bilayers on silicon dioxide.

Figure 4.13: Exemplary time-trace of a RIfS blind experiment.

Figure 4.14: Representative RIfS time-traces of the ENTH adsorption upon different protein concentrations to POPC/PIP 2 bilayers in (A) absence and (B) presence of PS.

Figure 4.15: Adsorption isotherms of ENTH to POPC/PIP2 (95:5) (blue circles) and POPC/POPS/PIP2 (75:20:5) (red circles) bilayers.

Figure 4.16: A representative force-distance curve measured on DOPC/DOPE/DOPS/PIP2/TxR (44.9:30:20:5:0.1)...

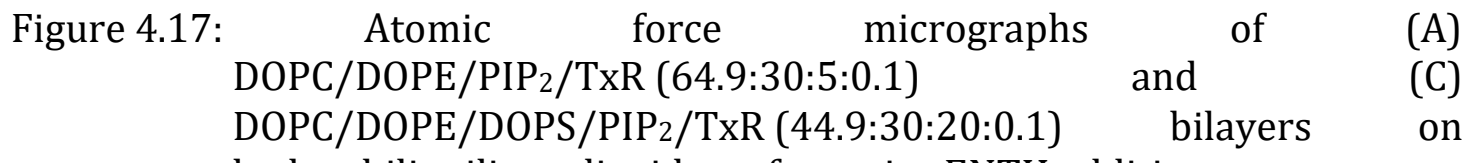

hydrophilic silicon dioxide wafers prior ENTH addition............................64 
Figure 4.18: Histogram plot of the protein heights averaged over 32 micrographs. 65

Figure 4.19: Fluorescence images of (A) DOPC/DOPE/PIP $2 / T x R$ (64.9:30:5:0.1) and (B) DOPC/DOPE/DOPS/PIP2/TxR (44.9:30:20:5:0.1) bilayers.

Figure 4.20: Time-resolved change in optical thickness upon addition of different ENTH R114A concentrations (marked by arrows) to (A) POPC/PIP2 (95:5) and (B) POPC/POPS/PIP 2 (75:20:5) bilayers

Figure 4.21: Adsorption isotherms of ENTH R114A to POPC/PIP2 (95:5) (blue circles) and POPC/POPS/PIP2 (75:20:5) (red circles) bilayers.

Figure 4.22: Atomic force micrographs of (A) DOPC/DOPE/PIP $/$ TxR $(64.9 / 30 / 5 / 0.1)$ and (C) DOPC/DOPE/DOPS/PIP $2 / T x R$ $(44.9 / 30 / 20 / 0.1)$ bilayers on hydrophilic silicon dioxide wafers prior protein addition.

Figure 4.23: Averaged surface pressure-area per molecule isotherms of POPC/POPE (80:20, black), POPC/POPE/PIP 2 (79:20:1, light blue), POPC/POPE/PIP2 (75:20:5, blue) and POPC/POPE/PIP2 (70:20:10, purple) at $20^{\circ} \mathrm{C}$.

Figure 4.24: Exemplary plot of the surface pressure vs. time of a POPC/POPE/PIP2 (75:20:5) monolayer.

Figure 4.25: Exemplary plot of the surface pressure vs. time of a POPC/POPE/PIP2 (70:20:10) monolayer after ENTH addition at $20^{\circ} \mathrm{C}$ using a protein trough.

Figure 4.26: Monolayer penetration of the ENTH domain into POPC/POPE (80:20) and POPC/POPE/PIP2 (80-x:20:x, with $\mathrm{x}=1,5,10)$ monolayers.

Figure 4.27: (A) Lipid packing against $\mathrm{PIP}_{2}$ concentration and (B) the critical surface pressure against the $\mathrm{PIP}_{2}$ content.

Figure 4.28: Averaged surface pressure-area per molecule isotherms of POPC/POPE (80:20, black, $n=4)$, POPC/POPE/PIP2 (75:20:5, blue, $n=3), \quad$ POPC/POPE/POPS $(60: 20: 20$, orange, $n=3)$ and

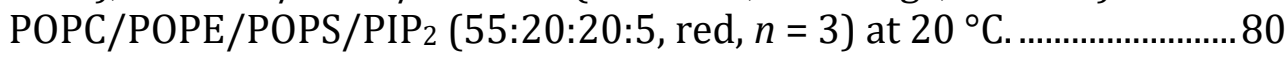

Figure 4.29: Monolayer penetration of the ENTH domain into POPC/POPE (80:20,), POPC/POPE/POPS (60:20:20), POPC/POPE/PIP2 (75:20:5) and POPC/POPE/POPS/PIP2 (55:20:20:5) monolayers

Figure 4.30: Schematic illustration of a GUV before (A) and after (B) adhesion to a surface.

Figure 4.31: Fluorescence images of (A) hydrophilized and (B) biotin-PEGfunctionalized glass slides after incubation with DyLight ${ }^{\circledR} 594$ labeled Neutravidin and subsequent rinsing with buffer. 
Figure 4.32: z-stacks of an adhered GUVs obtained by SDCLM.

Figure 4.33: Ratios of the contact area radius to the adhered vesicle radius $\left(r_{\mathrm{i}} / r_{\mathrm{ad}}\right)$ as a function of time for GUVs (DOPC/DOPS/Cholesterol/cap-biotinDOPE/PIP $/$ Atto488-DPPE (76:10:10:2:1:1)) adhered to a NeutrAvidin covered surface..

Figure 4.34: Lateral membrane tension of adhered GUVs on PEG-biotinNeutrAvidin functionalized glass substrates after 15 min and 2 h........ 92

Figure 4.35: Ratio of the contact area radius and the adhered vesicle radius $\left(r_{\mathrm{i}} / r_{\text {ad }}\right)$ of the adhered GUVs (DOPC/DOPS/Cholesterol/cap-biotinDOPE/PIP2/Atto488-DPPE (76:10:10:2:1:1)) immobilized on PEGNeutravidin functionalized glass.

Figure 4.36: Lateral membrane tension of adhered GUVs on PEG-NeutrAvidin functionalized glass substrates with different ratios of $m-P E G$ and $b-$ PEG used for immobilization of the GUVs.

Figure 4.37: Time series of one z-plane of an adhered GUV incubated with $1 \mu \mathrm{M}$ ENTH $(2 \mathrm{~h})$

Figure 4.38: Time series of one z-plane of an adhered GUV incubated with $0.3 \mu \mathrm{M}$ ENTH $(2 \mathrm{~h})$

Figure 5.1: Schematic illustration of the different interactions of lipids with substrate surfaces.

Figure 5.2: (A) Maximum change of the optical thickness upon ENTH binding to $\mathrm{PIP}_{2}$ doped mono-(green) and bilayers (black) dependent on the $\mathrm{PIP}_{2}$ content. (B) Schematic illustration of the $\mathrm{PIP}_{2}$ distribution within the mono- and bilayers and the influence on protein occupancy

Figure 5.3: Schematic illustration of the ENTH crowding mechanism 107

Figure 5.4: Cantilever movement in contact mode during imaging of (A) ENTH monomers and (B) ENTH clusters.

Figure 5.5: Atomic force micrographs of a DOPC/DOPE/DOPS/PIP2/TxR (44.9:30:20:5:0.1) bilayer after incubation with $1 \mu \mathrm{M}$ ENTH obtained by three different imaging modi: QI-, tapping and contact mode......... 110

Figure 5.6: Schematic illustration of the different interactions of (A) $\mathrm{IP}_{3}$ bound ENTH and ENTH bound to a $\mathrm{PIP}_{2}$ doped membrane.

Figure 5.7: Predicted protein heights of ENTH for different conditions.

Figure 5.8: Orientation and interactions of two ENTH monomers in the (A) absence and (B) presence of PS. 
Figure 5.9: Schematic illustration of the ENTH penetration into monolayers composed of POPC/POPE/PIP2 (70:20:10).

Figure 5.10: Illustration of $\pi_{\mathrm{c}}$ against the lipid packing at $\pi=20 \mathrm{mN} / \mathrm{m}$ for the lipid compositions POPC/POPE/PIP2 (80-x:20:x, with $\mathrm{x}=0$ or $5 \mathrm{~mol} \%$ ) and POPC/POPE/POPS/PIP 2 (60-x:20:20:x, with $\mathrm{x}=0$ or $5 \mathrm{~mol} \%)$

Figure 5.11: Schematic illustration of predicted ENTH-ENTH interactions resulting in protein clusters.

Figure 5.12: PEG-based surface functionalization.

Figure 5.13: Schematic illustration of the PEG-based surface functionalization. .... 128

Figure 5.14: Binding of ENTH (green) to adhered GUVs resulting in the formation of tubules 



\section{LiST OF TABLES}

Table 3.1: Used buffers and their compositions.

Table 4.1: Averaged optical and physical thicknesses of the POPC/PIP 2 lipid bilayers with the corresponding standard deviations.

Table 4.2: Mean values of the diffusion coefficients $D$ and the mobile fractions $F_{\mathrm{m}}$ of TxR in lipid bilayers with corresponding standard deviations yielded by FRAP experiments.

Table 4.3: The optical and physical thicknesses of the POPC/PIP 2 lipid monolayers with receptor lipid concentration ranging from 0-10 mol\%.....................48

Table 4.4: Mean values of the diffusion coefficients $D$ and the mobile fractions $F_{\mathrm{m}}$ of the fluorophores in lipid monolayers with corresponding standard deviations yielded by FRAP experiments.

Table 4.5: Mean values of the optical and physical thicknesses of the POPC/POPS (80:20) and POPC/POPS/PIP2 (75:20:5) lipid bilayers.

Table 4.6: Diffusion coefficients of TxR in DOPC/DOPE/PIP /TxR (64.9:30:5:0.1) and DOPC/DOPE/DOPS/PIP2/TxR (44.9:30:20:5:0.1) bilayers before and after the incubation of $1 \mu \mathrm{M}$ ENTH.

Table 4.7: Summary of the fit results of the Langmuir adsorption isotherms for ENTH wt and ENTH R114A.

Table 4.8: $A 20$ mean values with standard deviations obtained from the isotherms with the different lipid compositions.

Table 4.9: A20 mean values with standard deviations obtained from the isotherms with the different lipid compositions.

Table 5.1: Summary of the fit results of the Langmuir adsorption isotherms for ENTH wt and ENTH R114A.

Table 10.1 Averaged maximum changes in optical thickness after ENTH addition to $\mathrm{POPC} / \mathrm{PIP}_{2}$ bi- and monolayers with different $\mathrm{PIP}_{2}$ concentrations. 176

Table 10.2: Reversibly of the ENTH binding to POPC/PIP 2 bi- and monolayer in \%. The $\mathrm{PIP}_{2}$ content was varied from $1-10 \mathrm{~mol} \%$.

Table 10.3: Summary of all dissociation constants $K_{\mathrm{D}}$ of ENTH to $\mathrm{PIP}_{2}$ doped membranes found in literature. 



\section{APPENDIX}

\section{Devices}

\section{Atomic force microscopy}

Nanowizard 4

JPK Instruments AG, Berlin, GER

\section{Fluorescence microsopy}

LSM 880 Examiner

Carl Zeiss, Jena, GER

Objektiv WPlan APOChromat 63x/1.0

Carl Zeiss, Jena, GER

spinning disc confocal

custom build

custom stand based on ix73

Olympus, Tokyo, JAP

spinning disc Yokogawa CSU-X

Rota Yokogawa, Wehr, GER

camera iXON 897Ultra

Andor Technology Ltd., Belfast, UK

AOTF: TF525-250-6-3-GH18A

Gooch\&Housego PLC, Illminster, UK

piezo P-721-CDQ

Physik Instrumente,Karlsruhe, GER

filter wheel Rotr

Andor Technology Ltd

filter LP496 ET

AHF Analysentechnik,Tübingen,

GER

filter ET525/50

AHF Analysentechnik, Tübingen,

GER

objective CFI Plan 100XW

Nikon, Düsseldorf, GER

$100 \times / 1.1$

\section{Langmuir-Blodgett trough}

RK 1 Standard Langmuir Trough

Thermostat VWR
Riegler \& Kirstein GmbH, Potsdam, GER

VWR International, Darmstadt 


\section{Reflectometric Interference Spectroscopy (RIfS)}

NanoClalc-2000, SD2000 spectrometer

Flame Spectrometer

Halogen light source

Ismatec 795C peristaltic pump

\section{UV Vis Spectroscopy}

NanoDrop 2000c

\section{Electroformation}

Frequency generator Aglient 333220A

\section{Other devices}

Micro scale Satorius CP225D

Magnetic mixer MR $3001 \mathrm{~K}$

Osmometer

pH-meter Calimatic 766

Plasma cleaner Zepto

Thermomixer compact

Tip sonifier Sonoplus HD2070

Ultra sonic bath Sonorex RK $255 \mathrm{H}$

Ultrapure water system

Vacuum drying oven VD 23

Vortexer

NanoDrop 2000c
Ocean Optics, Dunedin, FL, USA

Ocean Optics, Dunedin, FL, USA

Ocean Optics, Dunedin, FL, USA

IDEX Health \& Science, Wertheim, GER

Thermo Scientific, Wilmington, USA

Aglient Technology, St. Clara, USA

Satorius, Göttingen, GER

Heidolph, Schwabach, GER

Knick, Berlin, GER

Diener Electronic, Ebbhausen, GER

Eppendorf, Hamburg, GER

Bandelin, Berlin, GER

Bandelin, Berlin

Millipore, Billerica, MA, USA

Binder, Tuttlingen, GER

Heidolph, Schwabach; GER

ThermoFisher Scientific, Waltham, MA, USA 


\section{Software}

ImageJ

Origin Pro 8.5G

ZEN black 2.3 SP1

MATLAB 2017b

Gwyddion 2.45

SpectraSuite

JPK Data Processing

RuK Trough Control http://rsbweb.nih.gov/ij

OriginLab Cooperation, Northhampton, USA

Carl Zeiss, Jena, GER

MathWorks, Natrick, MA, USA

http://gwyddion.net

Ocean Optics Germany GmBH, Ost-

fildern, GER

Instruments AG, Berlin, GER

Riegler \& Kirstein GmbH, Potsdam, GER

\section{Protein sequence}

The sequence of ENTH was obtained from the Protein Data Bank. ${ }^{23}$

1 HNYSEAEIKV REATSNDPWG PSSSLMSEIA DLTYNVVAFS EIMSMIWKRL

51 NDHGKNWRHV YKAMTLMEYL IKTGSERVSQ QCKENMYAVQ TLKDFQYVDR

101 DGKDQGVNVR EKAKQLVALL RDEDRLREER AHALKTKEKL AQTA 


\section{Optical thickness changes upon ENTH addition to POPC/PIP 2 bi- and monolayers}

Table 10.1 Averaged maximum changes in optical thickness after ENTH addition to POPC/PIP 2 bi- and monolayers with different $\mathrm{PIP}_{2}$ concentrations.

\begin{tabular}{|l|l|l|l|l|}
\hline $\begin{array}{l}\text { Lipid } \\
\text { Composition } \\
\text { POPC/PIP } 2\end{array}$ & bilayer & $\begin{array}{l}\text { Number of } \\
\text { measurements } \\
n\end{array}$ & $\begin{array}{l}\text { monolayer } \\
\Delta O T_{\text {ENTH }} / \mathrm{nm}\end{array}$ & $\begin{array}{l}\text { Number of } \\
\text { measurements } \\
n\end{array}$ \\
\hline $100: 0$ & $0.03 \pm 0.01$ & 5 & $0.06 \pm 0.04$ & 3 \\
\hline $99: 1$ & $0.1 \pm 0.1$ & 4 & $0.5 \pm 0.2$ & 4 \\
\hline $98: 2$ & $0.2 \pm 0.1$ & 5 & $0.8 \pm 0.3$ & 5 \\
\hline $95: 5$ & $0.5 \pm 0.2$ & 5 & $1.2 \pm 0.3$ & 4 \\
\hline $93: 7$ & $0.7 \pm 0.2$ & 4 & $1.7 \pm 0.6$ & 3 \\
\hline $92: 8$ & $0.9 \pm 0.2$ & 5 & $2.0 \pm 0.4$ & 3 \\
\hline $90: 10$ & $1.3 \pm 0.4$ & 8 & $2.2 \pm 0.5$ & 6 \\
\hline
\end{tabular}

\section{Reversibility of the ENTH binding to POPC/PIP 2 bi- and monolayers}

Table 10.2: Reversibly of the ENTH binding to POPC/PIP 2 bi- and monolayer in \%. The $\mathrm{PIP}_{2}$ content was varied from 1-10 mol\%. The errors are given as standard deviations.

\begin{tabular}{|l|l|l|}
\hline & Reversibility of the ENTH binding/ \% \\
\hline Lipid composition & Lipid bilayer & Lipid monolayer \\
\hline POPC/PIP 2 (99:1) & $70 \pm 40(n=4)$ & $83 \pm 15(n=4)$ \\
\hline POPC/PIP 2 (98:2) & $88 \pm 24(n=5)$ & $73 \pm 15(n=4)$ \\
\hline POPC/PIP2 (95:5) & $85 \pm 15(n=6)$ & $80 \pm 11(n=4)$ \\
\hline POPC/PIP2 (93:7) & $99 \pm 21(n=4)$ & $74 \pm 23(n=3)$ \\
\hline POPC/PIP2 (92:8) & $89 \pm 14(n=7)$ & $70 \pm 6(n=3)$ \\
\hline POPC/PIP2 (90:10) & $85 \pm 6(n=4)$ & $68 \pm 4(n=5)$ \\
\hline
\end{tabular}




\section{Dissociation constants of the ENTH binding to $\mathrm{PIP}_{2}$ containing mem- branes}

Table 10.3: Summary of all dissociation constants $K_{\mathrm{D}}$ of ENTH to $\mathrm{PIP}_{2}$ doped membranes found in literature.

\begin{tabular}{|c|c|c|}
\hline Binding assay & Lipid composition & $K_{\mathrm{D}}$ \\
\hline Liposome assay 155 & $\mathrm{PC} / \mathrm{PE} / \mathrm{PIP}_{2}(70: 20: 10)$ & $618.4 \mathrm{nM} \pm 0.009 \%$ \\
\hline $\mathrm{SPR}^{25}$ & $\begin{array}{l}\text { POPC/POPE/PIP } 2 \text { (77:20:3) } \\
\text { POPC/POPE/PIP } 2 \text { (79.5:20:0.5) }\end{array}$ & $\begin{array}{l}23 \pm 7 \mathrm{nM} \\
250 \pm 60 \mathrm{nM}\end{array}$ \\
\hline $\mathrm{SPR}^{8}$ & $\begin{array}{l}\text { POPC/POPE/PIP } 2(78: 20: 2) \\
\text { POPC/POPE/POPS/PIP2 (63:20:15:2) }\end{array}$ & $\begin{array}{l}80 \pm 11 \mathrm{nM} \\
20 \pm 2.4 \mathrm{nM}\end{array}$ \\
\hline $\mathrm{SPR}^{28}$ & POPC/POPS/PIP2 (77:20:3) & $49 \pm 8 \mathrm{nM}$ \\
\hline SPR $^{79}$ & $\begin{array}{l}\text { POPC/PIP2 (90:10) } \\
\text { POPC/POPE/PIP } 2 \text { (60:30:10) }\end{array}$ & $\begin{array}{l}0.5 \pm 0.1 \mu \mathrm{M} \\
0.69 \pm 0.08 \mu \mathrm{M}\end{array}$ \\
\hline RIfS 36,79 & $\mathrm{POPC} / \mathrm{PIP}_{2}(90: 10)$ & $0.8 \pm 0.2 \mu \mathrm{M}$ \\
\hline $\begin{array}{l}\text { Calorimetric titra- } \\
\text { tion }^{21}\end{array}$ & $\begin{array}{l}\text { PC/PE/Cholesterol/diC }{ }_{8} \mathrm{PIP}_{2} \\
(40: 40: 10: 10)\end{array}$ & $0.85 \mu \mathrm{M}$ \\
\hline $\begin{array}{l}\text { Fluorescence mi- } \\
\text { croscopy } 154\end{array}$ & DOPC/DOPS/PIP 2 TxRR $^{\circledR}(79: 15.5: 1)$ & $6 \mu \mathrm{M}$ \\
\hline $\begin{array}{l}\text { Fluorescence mi- } \\
\text { croscopy }^{79}\end{array}$ & DOPC/DOPE/PIP2 (60:30:10) & $0.5 \pm 0.2 \mu \mathrm{M}$ \\
\hline
\end{tabular}

\section{Abbrevations and symbols}

$\AA$

$A_{\mathrm{M}}$

$A_{\mathrm{M}, 20}$

$A_{\mathrm{v}}$

$A_{\text {ad }}$

AFM

b-PEG

b-PEG-NH2
Angström

Area per molecule

Area per molecule at $20 \mathrm{mN} / \mathrm{m}$

Area of a free giant unilamellar vesicle

Are of an adhered giant unilamellar vesicle

Atomic force microscopy

Biotin polyethylene glycol

Biotin polyethylene glycol amine 


\begin{tabular}{|c|c|}
\hline BSA & Bovine serum albumin \\
\hline $\mathrm{C}$ & Concentration \\
\hline $\mathrm{CCV}$ & Clathrin-coated vesicles \\
\hline Cf. & compare \\
\hline CG-MD & Coarse-grained molecular dynamics \\
\hline CLSM & Confocal laser scanning microscopy \\
\hline CME & Clathrin-mediated endocytosis \\
\hline$D$ & Diffusion coefficient \\
\hline$d$ & Cuvette thickness \\
\hline$d_{\mathrm{M}}$ & Physical membrane thickness \\
\hline$d_{\text {ENTH }}$ & Physical thickness of ENTH \\
\hline DHPE & 1,2 Dihexadecanoyl-sn-glycero-3-phosphoethanolamin \\
\hline DIPS & 1,2-dilinoleoyl-sn-glycero-3-phospho- $L$-serine \\
\hline DPPE & 1,2-dipalmitoyl-sn-glycero-3-phosphoethanolamine \\
\hline DOPC & 1,2-dioleoyl-sn-glycero-3-phosphocholine \\
\hline DOPE & 1,2-dioleoyl-sn-glycero-3-phosphoethanolamine \\
\hline DOPS & 1,2-dioleoyl-sn-glycero-3-phospho- $L$-serine \\
\hline DPPS & 1,2-dipalmitoyl-sn-glycero-3-phospho- $L$-serine \\
\hline EDTA & Ethylenediaminetetraacetic acid \\
\hline EGFR & Epidermal growth factor receptor \\
\hline ENTH & Epsin $N$-terminal Homology \\
\hline EPR & Electron paramagnetic resonance \\
\hline$F$ & Force \\
\hline$F_{\mathrm{m}}$ & Mobile fraction \\
\hline FRAP & Flourescence recovery after photobleaching \\
\hline FWHM & Full width at half maximum \\
\hline GOPTS & (3-Glycidyloxypropyl)trimethoxysilane \\
\hline GUV & Giant unilamellar vesicle \\
\hline$I$ & Intensity \\
\hline$h$ & Height \\
\hline h & Hour \\
\hline $\mathrm{H}_{2} \mathrm{O}$ & Water \\
\hline
\end{tabular}




\begin{tabular}{|c|c|}
\hline $\mathrm{H}_{2} \mathrm{O}_{2}$ & Hydrogen peroxide \\
\hline HMDS & Hexamethyldisilane \\
\hline HEPES & (4-(2-hydroxyethyl)-1-piperazine)-ethanesulfonic acid \\
\hline $\mathrm{Hz}$ & Hertz \\
\hline ITO & Indium tin oxide \\
\hline$k$ & Spring constant \\
\hline$K_{\mathrm{A}}$ & Area compressibility modulus \\
\hline $\mathrm{KCl}$ & Potassium chloride \\
\hline$K_{\mathrm{D}}$ & Dissociation constant \\
\hline $\mathrm{kDa}$ & Kilodalton \\
\hline $\mathrm{KH}_{2} \mathrm{PO}_{4}$ & Monopottasium phosphate \\
\hline LC & Liquid-condensed \\
\hline LE & Liquid-expanded \\
\hline LUV & Large unilamellar vesicle \\
\hline M & Molecular mass \\
\hline $\mathrm{MeOH}$ & Methanol \\
\hline $\mathrm{MgCl}_{2}$ & Magnesium chloride \\
\hline Min & Minute \\
\hline MLV & Multilamellar veicles \\
\hline m-PEG & Methoxy polyethylene glycol \\
\hline m-PEG-NH2 & Methoxy polyethylene glycol amine \\
\hline$n$ & Refractive index, number of measurements \\
\hline$N A$ & Numerical aperture \\
\hline $\mathrm{nm}$ & Nanometer \\
\hline $\mathrm{Na}$ & Sodium \\
\hline $\mathrm{NaCl}$ & Sodium chloride \\
\hline $\mathrm{Na}_{2} \mathrm{HPO}_{4}$ & Disodium phosphate \\
\hline $\mathrm{NaN}_{3}$ & Sodium azide \\
\hline $\mathrm{NH}_{3}$ & Ammonia \\
\hline OT & Optical thickness \\
\hline PBS & Phosphate buffered saline \\
\hline PEG & Polyethylene glycol \\
\hline
\end{tabular}




\begin{tabular}{|c|c|}
\hline PEM & Photoelastic modulator \\
\hline $\mathrm{PIP}_{2}$ & Phosphatidylinositol-4,5-bisphosphate \\
\hline POPC & 1-Palmitoyl-2-oleoyl-sn-glycero-3-phosphocholine \\
\hline POPE & 1-Palmitoyl-2-oleoyl-sn-glycero-3-phosphoethanolamine \\
\hline POPS & 1-Palmitoyl-2-oleoyl-sn-glycero-3-phosph \\
\hline$r \mathrm{ad}$ & Radius of an adhered giant unilamellar vesicle \\
\hline$r_{\mathrm{i}}$ & Radius of the contact area \\
\hline$r_{\mathrm{v}}$ & Radius of a free giant unilamellar vesicle \\
\hline$r_{1 / 2}$ & Fresnel coefficients \\
\hline RIfS & Reflectometric interference ppectroscopy \\
\hline rms & Root mean square \\
\hline ROI & Region of interest \\
\hline rpm & Revolutions per minute \\
\hline RT & Room temperature \\
\hline s & Second \\
\hline$S$ & Traveling distance \\
\hline SAM & Self assembled monolayer \\
\hline SD & Standard deviation \\
\hline SDCLM & Spinning disc confocal laser microscopy \\
\hline SLB & Supported lipid bilayer \\
\hline $\mathrm{SiO}_{2}$ & Silicon dioxide \\
\hline SPR & Surface plasmon resonance \\
\hline SUV & Small unilamellar vesicle \\
\hline$t$ & Time \\
\hline$T$ & Temperature \\
\hline $\mathrm{TiO}_{2}$ & Titanium dioxide \\
\hline U & Voltage \\
\hline UV & Ultraviolett \\
\hline V & Volt \\
\hline$V$ & Volume \\
\hline Vis & Visible \\
\hline wt & Wild type \\
\hline
\end{tabular}




$\begin{array}{ll}\mathrm{Zc} & \text { Vertical deflection } \\ \mathrm{Z}_{\mathrm{p}} & \text { Piezo position } \\ \alpha & \text { Angle of incident light } \\ \gamma & \text { Surface tension } \\ \delta & \text { Phasendifferenz } \\ \kappa & \text { Bending rigidity } \\ \varphi & \text { Angle of refracting light } \\ \theta & \text { Contact angle } \\ \lambda & \text { Wavelength } \\ \sigma & \text { Membrane tension } \\ \pi & \text { Surface pressure } \\ \pi_{\mathrm{c}} & \text { Critical surface pressure } \\ \pi_{0} & \text { Initial surface pressure } \\ \chi & \text { Molar fraction }\end{array}$

\section{Materials}

Braungläschen

Cantilever

Eppendorf cups

Eppendorf pipettes

Hamilton syringe

ITO-slides

Klemmen

Copper stripes

Menzel-Objektträgergläschen

Parafilm

Petri dishes

Pipette tips

Pump tubes Tygon

RIfS chamber

$\mathrm{SiO}_{2}$ substrates
OCHS GmbH, Göttingen, GER

Eppendorf, Hamburg, GER

Eppendorf, Hamburg, GER

ThermoScientific, Braunschweig, GER

Präzisions Glas \& Optik GmbH, Isersloh, GER

ALCO, Arnsberg, GER

Präzisions Glas \& Optik GmbH, Isersloh, GER

ThermoScientific, Braunschweig, GER

American National Can, Chicago, GER

Sarstedt, Nürnbrecht, GER

Sarstedt, Nürnbrecht, GER

Ismatec, Wertheim-Mondfeld, GER

custom-built, OC Werkstatt, Göttingen, GER

Sitron Inc, KOR 


\section{Chemicals}

Aceton

Carl Roth, Karlsruhe, GER

Dylight $^{\circledR} 594$ NeutrAvidin

Thermo Fisher, Waltham,USA

Aluminiumoxid

Sigma-Aldrich, Taufkirchen, GER

Ammonia solution (25\%)

VWR International, Darmstadt, GER

Argon

Linde, München, GER

Biotin-PEG-NH2

Thermo Fisher, Waltham, USA

BSA. Protease free

Carl Roth, Karlsruhe, GER

Chloroform

VWR International, Darmstadt, GER

Cholesterol

Sigma-Aldrich, Taufkirchen, GER

DOPC

Avanti Polar Lipids, Alabaster, USA

DOPE

Avanti Polar Lipids, Alabaster, USA

DOPS

Avanti Polar Lipids, Alabaster, USA

Ethanol, p.a.

VWR International, Darmstadt, GER

Glucose

Carl Roth GmbH, Karlsruhe, GER

GOPTS

Sigma Aldrich, Taufkirchen, GER

HEPES

Carl Roth GmbH, Karlsruhe, GER

$\mathrm{H}_{2} \mathrm{O}_{2}(30 \%)$

Grüssing GmbH, Filsum, GER

$\mathrm{KCl}$

Carl Roth GmbH, Karlsruhe, GER

$\mathrm{KH}_{2} \mathrm{PO}_{4}$

Merck, Darmstadt, GER

$\mathrm{KOH}$

Merck, Darmstadt, GER

Methanol

VWR International, Darmstadt, GER

$\mathrm{MgCl}_{2} \cdot 6 \mathrm{H}_{2} \mathrm{O}$

Merck, Darmstadt, GER

Mucasol $^{\circledR}$

Sigma-Aldrich, Taufkirchen, GER

$\mathrm{NaCl}$

Merck, Darmstadt, GER

Na citrate

Merck, Darmstadt, GER

$\mathrm{NaN}_{3}$

Merck, Darmstadt, GER

$\mathrm{Na}_{2} \mathrm{HPO}_{4}$

VWR International, Darmstadt, GER

NeutrAvidin

Thermo Fisher, Waltham, USA 
Nitrogen

Parafilm

Pipette tips

$\mathrm{PIP}_{2}$

POPC

POPE

POPS

Hydrochlorid acid

Sucrose

TxR
Linde, München, GER

Pechiney Plastic Packaging, Chicago, USA

Sarstedt, Nürnbrecht, GER

Avanti Polar Lipids, Alabaster, USA

Avanti Polar Lipids, Alabaster, USA

Avanti Polar Lipids, Alabaster, USA

Avanti Polar Lipids, Alabaster, USA

Merck, Darmstadt, GER

Carl Roth GmbH, Karlsruhe, GER

Sigma-Aldrich, Taufkirchen, GER 



\section{Curriculum vitae}

\section{Personal data}

Name: $\quad$ Nelli Teske

Date of birth: $\quad 01.01 .1990$

Place of birth: Karabulak, Kasachstan

Citizenship: German

\section{Education}

2015-2019

PhD thesis at the Georg-August-University Göttingen (Prof. Dr. C. Steinem), Title: Lipid dependent interactions of biomimetic membrane models with the ENTH domain of epsin.

2013-2015

2010-2013

Graduate studies in chemistry at the Georg-August-University Göttingen (Prof. Dr. C. Steinem). Title of master thesis: Charakterisierung von Funktionalisierungsstrategien zur Verminderung der abstandsabhängigen Fluoreszenzlöschung auf Goldoberflächen

Undergraduate studies in chemistry at the Georg.-August-University Göttingen(Prof. Dr. C. Steinem). Title of bachelor thesis: Orientierte Rekonstitution von Bacteriorhodopsin in artifizielle Membransysteme

2009-2010 Undergraduate studies in mathematics at the Georg-AugustUniversity Göttingen.

2006-2009 Allgemeine Hochschulreife at the Gymnasium Athenaeum Stade 


\section{Scientific contributions}

\section{publications}

2019

Kroppen, B, Teske, N., Kondratiuk, I., Denkert, N., Mukherjee, I., Yambire K. F., Jaipuria, G, Zweckstetter, M., Milosevic, I., Steinem, C., Meinecke M. (2019) Lipid-dependent oligomerization of the epsin1 ENTH domain drives membrane deformation and is important for clathrin-mediated endocytosis, in preparation.

Teske, N. \& Sibold, J., Schumacher, J., Teiwes, N. K., Gleisner, M., Mey, I., Steinem, C. (2017) Continuous pore-spanning lipid bilayers on silicon oxide-coated porous substrates. Langmuir 2017, 33, 14175-14183.

Gleisner, M., Kroppen, B., Fricke, C., Teske, N., Kliesch, T.-T., Janshoff, A., Meinecke, M., Steinem, C. (2016) Epsin N-terminal homology domain (ENTH) activity as a function of membrane tension. J. Biol. Chem. 2016, 291, 19953-19961.

\section{oral presentations}

SFB 803 Winter school. Lipid dependent ENTH-membrane interactions.

SFB 803 Autumn school. Continuous pore-spanning bilayers on silicon oxide coated porous substrates.

\section{Selected poster presentations}

Biennial Meeting of the German Biophysical Society, Düsseldorf. Investigation of biomimetic membrane interactions with the ENTH domain of epsin 1 and substrate surfaces. 
$19^{\text {th }}$ IUPAB and $11^{\text {th }}$ EBSA Congress, Edinburgh. Interactions of biomimetic membrane models with the ENTH domain of epsin 1 and substrate surfaces.

2016 International Symposium "Biological Dynamics from Microscopic to Mesoscopic Scales",Grimma. Measurements of ENTH activities in lipid membrane systems. 



\section{Danksagung}

Zunächst danke ich Prof. Dr. Claudia Steinem für die wissenschaftliche Betreuung dieser Doktorarbeit. Die vielen hilfreichen Diskussionen haben mich immer wieder motiviert und vorangebracht.

Danke auch an Prof. Dr. Burg für die Übernahmen des Korreferats. Zudem bedanke ich mich für die konstruktiven Ratschläge in den Thesis Committees.

Bei Prof. Dr. Michael Meinecke möchte ich mich für die gute Kooperation, die Teilnahme an meinem Thesis Committee und die daraus resultierenden wertvollen Ideen und Diskussionen bedanken.

Ein ganz besonderer Dank geht an Ingo, der mir in vielen wissenschaftlichen Fragen und sonstigen Problemen geholfen hat. Manchmal kannst du ja doch nett sein. Dank dir weiß ich nun auch wie einfach das Zubereiten von Pulled Pork ist..., aber immerhin sind wir jetzt quitt!

Ebenfalls danke ich Tabea für die Unterstützung bei Problemen und Fragen während der Doktorarbeit.

Vielen, vielen Dank auch an Melanie, Marianne und Dana für ihre Hilfe in allen organisatorischen Fragen und für all die lustigen Gespräche im Sekretariat. Danke für eure Geduld bei allen Formularen, Raumbuchungen oder sonstigen Terminplanungen. Das hat das Arbeiten deutlich erleichtert.

Jutta und Micha möchte ich für die Unterstützung bei allen Laborarbeiten danken. Danke insbesondere für die teilweise sehr spontanen Experimentvorbereitungen. Ihr habt das Arbeiten schnell und angenehm gemacht. Zudem danke ich Micha und HansPeter für die tolle Unterstützung im Mediziner-Praktikum.

Dr. Benjamin Kroppen und Indrani Mukherjee danke ich für die gute Kooperation und die Proteinisolation.

Danke auch an meine Leidensgenossen Johannes und Jerry im Schreibraum. Auch wenn ich eure MatLab Gespräche nicht unbedingt vermissen werde, war es sehr lustig mit euch. Vor allem danke ich Jerry dafür, dass er mich nicht wegen meiner tausend Fragen, Diskussionen oder Beschwerden aus dem Fenster geworfen hat. Verdient hätte ich es und dass du das hingekriegt hättest, ist auch kein Geheimnis. 
Bei meinen Bachelorstudenten Elisa, Stephan und Patrick möchte ich mich für ihren Beitrag zu dieser Arbeiten bedanken. Eure Beiträge haben meine Arbeit erheblich vorangebracht.

Weiterhin möchte ich mich bei dem hervorragenden asiatischen Lieferservice von Buddy während der Schreibphase bedanken. Es ist super, wenn man sich um eine Sache weniger sorgen muss. Auch danke ich Nils (und Kri) für die diversen Mahlzeiten. Ich stehe immer gerne für eine Kaiserschmarrn-Verkostung (oder ein Sch(l)äfchen) zur Verfügung. Und natürlich darf ich Niko nicht vergessen. Danke für das tägliche „Gießen“. Ohne dich wäre ich bestimm schon längst „verwelkt“.

Ich danke allen Korrekturlesern, insbesondere Jerry, Peter und Jonas, die noch einige interessante Ideen und Aspekte zu dieser Arbeit beigesteuert haben.

Dem ganzen AK möchte ich für alle sinnvollen und weniger sinnvollen Gespräche während und nach der Arbeitszeit bedanken. Ich werde unsere Grill-, Karaoke- oder sonstigen Abende sehr vermissen! UND das hab ich (gar nicht) so gemeint!!!

Ein weiterer Dank gilt meinen Kommilitonen und Freunden, die das Studentenleben unvergesslich gemacht haben. Ein großer Dank geht vor allem an meine Mädels. Auch wenn wir mittlerweile etwas verstreut leben, die Tages- und Abendausflüge bleiben die besten Ablenkungen von einem anstrengenden Tag. Ihr seid die Besten!

Zum Schluss bedanke ich mich von ganzem Herzen bei meiner Familie. Danke, dass ihr mir das alles ermöglicht und mich immer unterstützt habt. Das hier ist auch euer Verdienst. Danke für alles! 